\title{
INL Fleet Vehicle Characterization Study for the U.S. Department of Navy
}

Brion Bennett Jim Francfort

September 2015

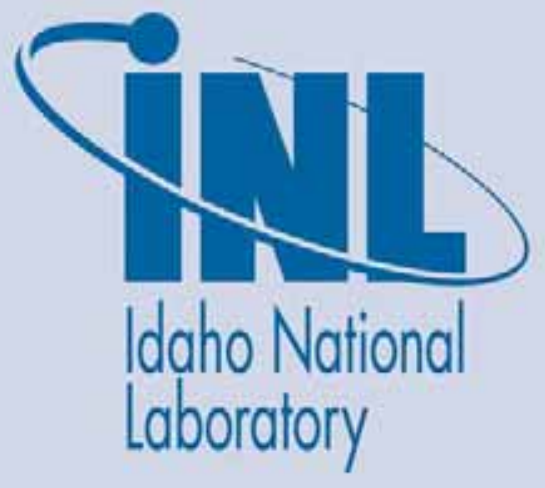

The INL is a U.S. Department of Energy National Laboratory operated by Battelle Energy Alliance 


\section{DISCLAIMER}

This information was prepared as an account of work sponsored by an agency of the U.S. Government. Neither the U.S. Government nor any agency thereof, nor any of their employees, makes any warranty, expressed or implied, or assumes any legal liability or responsibility for the accuracy, completeness, or usefulness, of any information, apparatus, product, or process disclosed, or represents that its use would not infringe privately owned rights. References herein to any specific commercial product, process, or service by trade name, trade mark, manufacturer, or otherwise, does not necessarily constitute or imply its endorsement, recommendation, or favoring by the U.S. Government or any agency thereof. The views and opinions of authors expressed herein do not necessarily state or reflect those of the U.S. Government or any agency thereof. 
INL/EXT-15-36439

\title{
INL Fleet Vehicle Characterization Study for the U.S. Department of Navy
}

\author{
Brion Bennett ${ }^{1}$ \\ Jim Francfort ${ }^{2}$ \\ ${ }^{1}$ Vehicle Systems Project Engineer, Idaho National Laboratory \\ ${ }^{2}$ Vehicle Systems Project Manager, Idaho National Laboratory
}

September 2015

Idaho National Laboratory Idaho Falls, Idaho 83415

http://avt.inl.gov

Prepared for the

U.S. Department of Energy

Office of Nuclear Energy

Under DOE Idaho Operations Office

Contract DE-AC07-05ID14517 


\section{ABSTRACT}

Idaho National Laboratory worked with the U.S. Department of Navy to collect and evaluate data from federal fleet vehicle operations in the San Diego area. This study collected and evaluated data to validate the introduction and use of advanced battery electric vehicles (BEV) in fleet applications.

Findings are reported on vehicle and mission characterizations to support the successful introduction of BEVs into military base fleets.

Individual observations of these vehicles provide the basis for recommendations related to BEV adoption and whether a BEV can fulfill the mission requirements as currently defined. 


\section{CONTENTS}

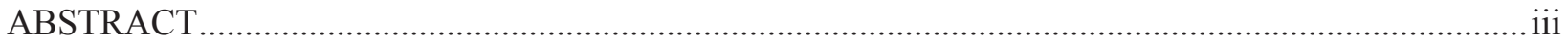

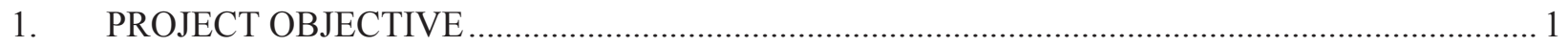

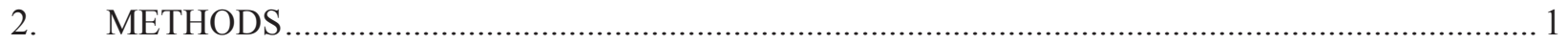

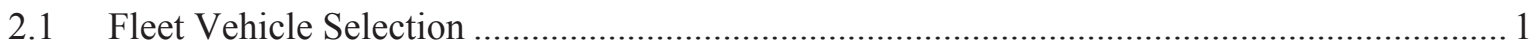

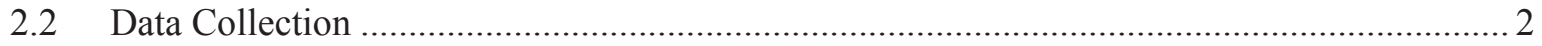

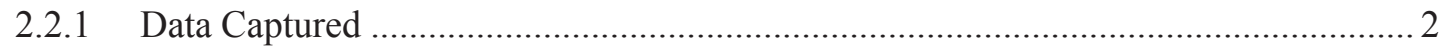

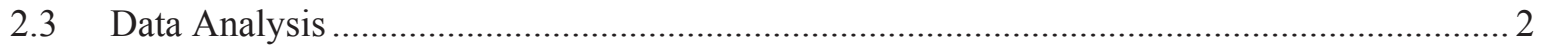

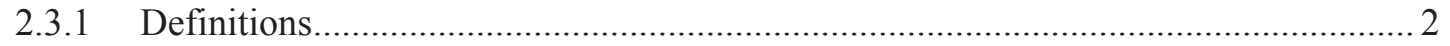

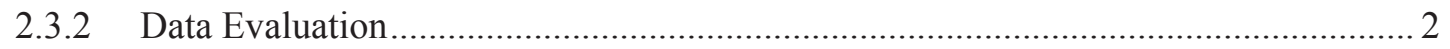

2.3.3 Battery Electric Vehicle Range Estimates ......................................................... 2

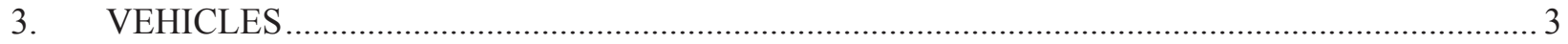

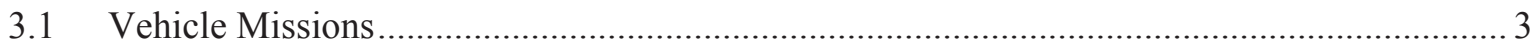

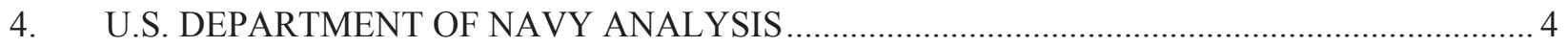

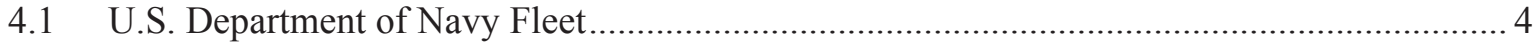

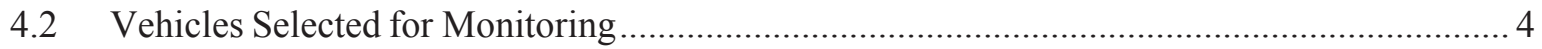

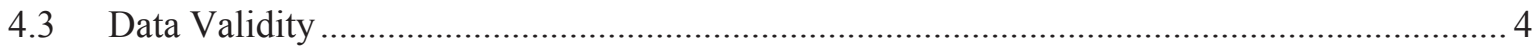

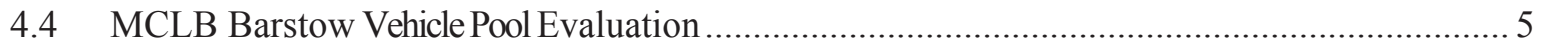

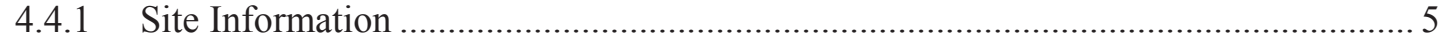

4.4.2 Summary for Pool Vehicles ................................................................................. 5

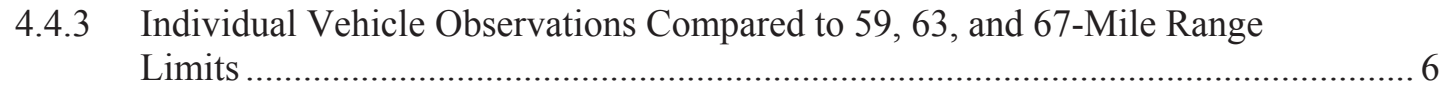

4.5 MCB Camp Pendleton Vehicle Pool Evaluation ................................................................. 7

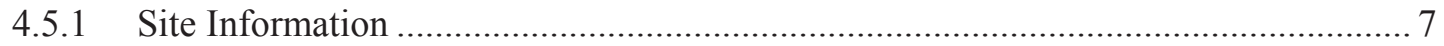

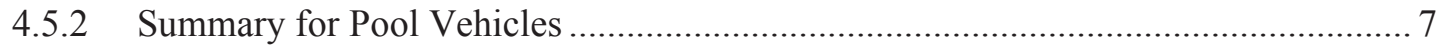

4.5.3 Vehicle Observations Compared to 63-Mile Range Limit......................................... 10

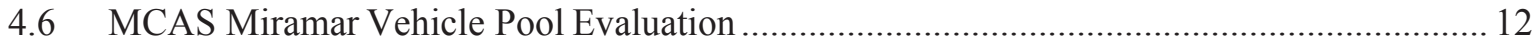

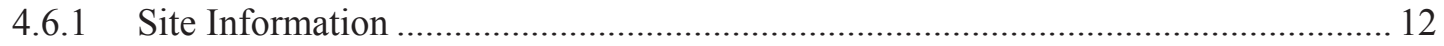

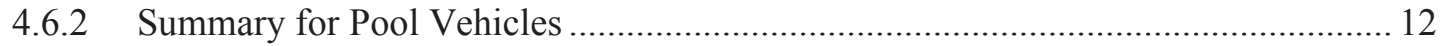

4.6.3 Vehicle Observations Compared to a 63-Mile Range Limit...................................... 14

4.7 MCAGCC 29 Palms Vehicle Pool Evaluation ..................................................................... 14

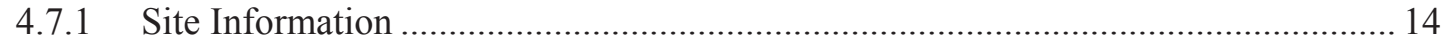

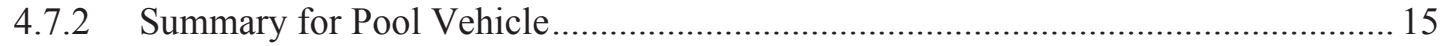

4.7.3 Individual Vehicle Observation(s) Compared to 59, 63, and 67-Mile Range Limits 


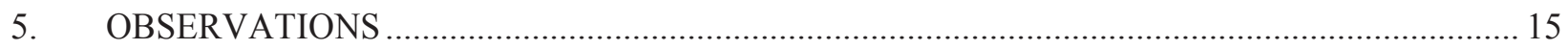

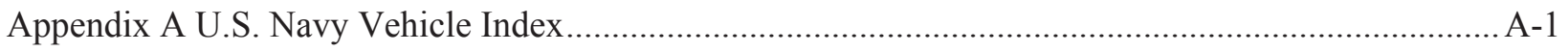

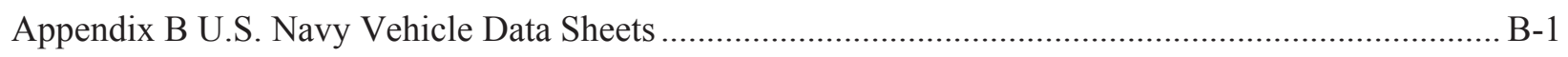

\section{TABLES}

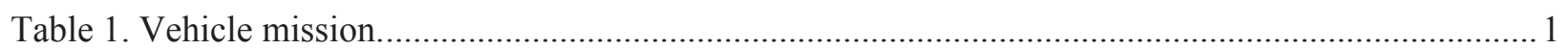

Table 2. Estimated real-world BEV range at 5 years and approximately 50,000 miles. .......................... 3

Table 3. Navy fleet vehicles by U.S. Environmental Protection Agency type.......................................... 4

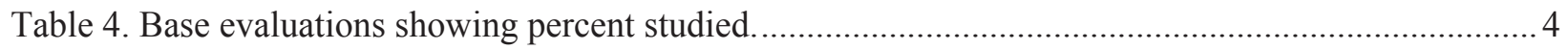

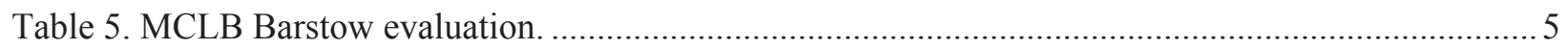

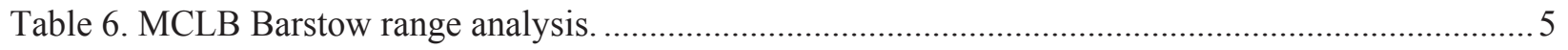

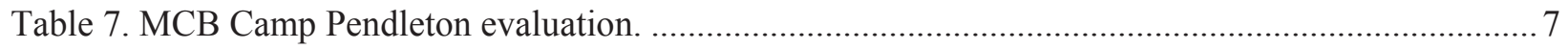

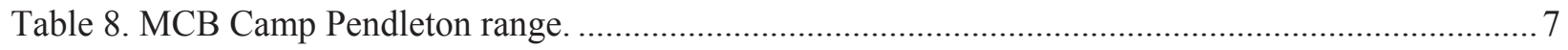

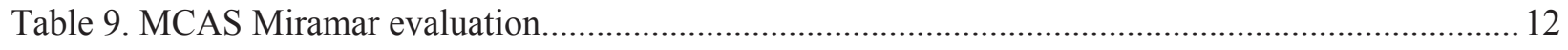

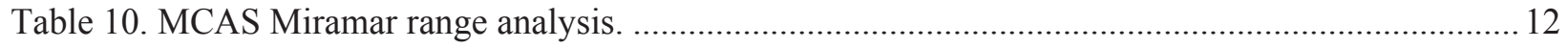

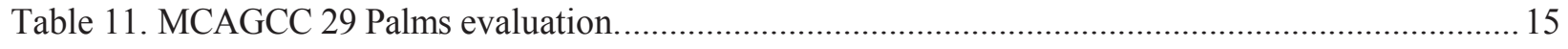

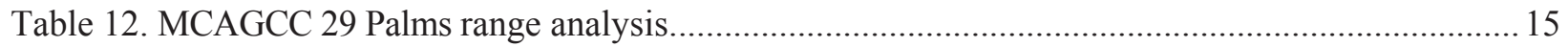

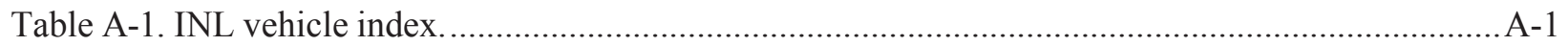




\section{INL Fleet Vehicle Characterization Study for the U.S. Department of Navy}

\section{PROJECT OBJECTIVE}

The objective of Idaho National Laboratory study for the U.S. Department of Navy was to perform analysis on light-duty internal combustion engine (ICE) vehicles that are operating in four United States Marine Corps fleets in order to identify candidate vehicles for replacement by battery electric vehicles (BEVs). This report documents the results of this study.

The above objective was accomplished by conducting the following four tasks:

1. Data collection: Coordinated with the fleet manager to collect data on agency fleet vehicles. Data were provided to Idaho National Laboratory (INL) via the fleet management tool, Networkfleet ${ }^{\circledR}$, and consisted of an activity detail report and the stop detail reports, which included vehicle trip data.

2. Data analysis and review: Examined data collected by data loggers and fleet vehicle characteristics to describe typical fleet activity.

3. Battery electric vehicle (BEV) implementation feedback: Provide feedback to fleet personnel and the U.S. Navy on selection criteria for replacement BEVs in their specific fleet vehicle missions.

4. Observations and recommendations: Provide actionable information to introduce BEVs into agency fleet operations.

Data collected or received from the ICE vehicles include trip distance, idle time, time between uses, and parking locations. The reports provided data at 2-minute intervals and the collection period for this analysis was from August 1, 2014, to July 1, 2015.

Fleet managers may use the information supplied in this report to help them identify which vehicles are candidates for replacement by BEVs based on historical use. BEVs are preferred because of the greater potential reduction of greenhouse gas emissions, fuel cost, and petroleum usage, but they are not likely to be suitable for all vehicle missions.

\section{METHODS}

\subsection{Fleet Vehicle Selection}

Agency fleet managers selected fleet vehicles for this study and provided basic information for each vehicle, including its managing base, primary vehicle mission, vehicle make, model, and model year.

The U.S. Navy identified 73 vehicles in their fleet for this assessment (Table 1). The vehicles span four operational Marine Corps installations (i.e., Marine Corps Logistics Base (MCLB) Barstow, Marine Corps Base (MCB) Camp Pendleton, Marine Corps Air Station (MCAS) Miramar, and Marine Corps Air Ground Combat Center (MCAGCC) 29 Palms). The fleet manager assessed the wide range of vehicles and made selections of high-interest, representative vehicles based on vehicle missions and vehicle type/class.

Table 1. Vehicle mission.

Vehicle Mission

MCLB Barstow

MCB Camp Pendleton

MCAS Miramar

MCAGCC 29 Palms

\section{Study Vehicles}

5

53

14 


\subsection{Data Collection}

Data collection occurred by vehicle identification, which was identified by base operations and a vehicle identification number or agency-assigned vehicle number. INL received no information related to the vehicle operator and provided no raw data to fleet managers. In this manner, INL did not collect, analyze, or report on driving habits of individual drivers.

\subsubsection{Data Captured}

Data consisted of key-on events, key-off events, and location, which were logged approximately every 2 minutes while the vehicle was on.

From these data points, the following information was available for evaluation:

- Trip start and stop time and location

- Trip distance and duration

- Idle start time, location, and duration

- Stop start time, location, and duration.

\subsection{Data Analysis}

\subsubsection{Definitions}

INL analyzed the vehicle's activity detail to determine the vehicle's daily usage, which was comprised of trips, stops, and idle events that occurred during a day. The following list provides definitions of these terms:

1. Trip: A trip begin with a key-on event and ended with the next key-off event.

2. Parking event: A vehicle parking event included the time between the key-off of the previous trip to the key-on of the next trip.

3. Idle time: Idle time was the amount of time a vehicle spends stationary after a key-on event when the vehicle was not moving for a period of 3 minutes or longer.

4. Trip travel time: Trip travel time was the amount of time between key-on and the next key-off, including idle time.

\subsubsection{Data Evaluation}

Processing data involved removal of null values and aggregation by different spatial and temporal scales. Aggregation was by day and trip to produce figures showing the patterns of use. Section 4 presents these results. INL observations are included in Section 5.

Statistical data analysis uses Microsoft ${ }^{\circledR}$ Excel, SQL Server 2012, and Matlab ${ }^{\circledR}$ software. Frequency distributions summarize the travel behavior of each vehicle and vehicle mission during the study period. Rounding of the tables and figures are to three significant digits.

\subsubsection{Battery Electric Vehicle Range Estimates}

In order to determine if existing ICE vehicles in the Marine Corps fleets can be replaced by BEVs, INL had to establish the likely range of BEVs being offered during model year 2015. The Model Year 2015 Fuel Economy Guide ${ }^{a}$ lists BEVs the U.S. Navy may procure with ranges from 82 miles (Chevrolet Spark EV) to 93 miles (Kia Soul Electric). A mid-point of 87.5 miles per charge was assumed (Table 2). However, these ranges were established without the real-world use of accessory loads (e.g., cabin

\footnotetext{
${ }^{\text {a } h t t p s: / / w w w . f u e l e c o n o m y . g o v / f e g / p d f s / g u i d e s / F E G 2015 . p d f ~}$
} 
comfort) that drivers demand, and it is known that battery range is reduced over time. Therefore, INL used the method described as follows to estimate BEV range after approximately 5 years of use.

Table 2. Estimated real-world BEV range at 5 years and approximately 50,000 miles.

\begin{tabular}{lccc} 
Model & $\begin{array}{c}\text { Fuel Economy } \\
\text { Guide Range }\end{array}$ & $\begin{array}{c}\text { Estimated Range with } \\
\text { Accessories (15\% Reduction) }\end{array}$ & $\begin{array}{c}\text { Estimated Range at } \\
\text { 5 Years (15\% Reduction) }\end{array}$ \\
Chevrolet Spark & 82 & 69.7 & 59 \\
Midpoint & 87.5 & 74.4 & 63 \\
Kia Soul & 93 & 79.05 & 67 \\
\hline
\end{tabular}

INL testing of BEVs has established that one test cannot be representative of the range every driver in every section of the United States will achieve. Testing by INL of the 2013 Nissan Leaf ${ }^{b}$ and the 2013 Ford Focus $^{\mathrm{c}}$ demonstrated energy efficiencies of $133.4 \mathrm{Wh} / \mathrm{mile}$ to $458.7 \mathrm{Wh} / \mathrm{mile}$ for the Leaf. This translates to 7.5 to $2.2 \mathrm{~kW} /$ mile. The variation is due to temperatures and speeds when testing. In addition, Nissan Leafs (with between 45,000 and 55,000 miles) operating as part of The EV Project in Los Angeles and San Diego were examined during 1,645 trips; they had an average range of 64.9 miles.

Based on the 6 months of operating data INL received, the internal combustion engine vehicles in the Marine Corps fleets averaged between 45,000 and 55,000 miles over a 5-year period. Therefore, The EV Project's 64.9-mile range appears reasonable when compared to the Table 2 calculations. As another example, range testing conducted by INL for Leafs charged at normal Level 2 charge infrastructure experienced range reductions of $21 \%$, on average, at 50,000 test miles. ${ }^{\mathrm{d}}$ Use of the $15 \%$ reduction for capacity fade over 50,000 miles seems to be a conservative estimate given that the areas the Marine BEVS will be operated in will not be as hot as the area the INL-tested Leafs operated in. Many other range reduction results can be referenced if needed.

\section{VEHICLES}

\subsection{Vehicle Missions}

Vehicle mission is an important characteristic in the fleet study. Based on information provided by the Marine Corps fleet managers, INL presumes that all vehicles in this study are pool vehicles. The pool vehicles in this study consisted of compact sedans, midsize sedans, small trucks, and full-size trucks.

\footnotetext{
${ }^{\mathrm{b}}$ http://avt.inl.gov/pdf/fsev/fact2013nissanleaf.pdf

${ }^{\mathrm{c}}$ http://avt.inl.gov/pdf/fsev/fact2013fordfocus.pdf

${ }^{\mathrm{d}} \mathrm{http}$ //avt.inl.gov/pdf/prog_info/IEA_DCFCImpactStudySept2014.pdf
} 


\section{U.S. DEPARTMENT OF NAVY ANALYSIS 4.1 U.S. Department of Navy Fleet}

The U.S. Navy provided reports for 73 vehicles in their fleets at four operating bases in the San Diego mission area. Table 3 shows the breakdown of these vehicles by vehicle type and by base.

Table 3. Navy fleet vehicles by U.S. Environmental Protection Agency type.

\begin{tabular}{llccccccc}
\hline \multicolumn{1}{c}{ Department } & \multicolumn{1}{c}{$\begin{array}{c}\text { Sedan } \\
\text { Description }\end{array}$} & $\begin{array}{c}\text { Van } \\
\text { Midsize }\end{array}$ & SUV & Minivan & Cargo & Pass & Truck & Total \\
\hline MCLB Barstow & Marine Corps Logistics Base & 1 & - & 1 & - & 1 & 2 & 5 \\
MCB Camp Pendleton & $\begin{array}{l}\text { Marine Corps Base Camp } \\
\text { Pendleton }\end{array}$ & 14 & - & 7 & - & 12 & 20 & 53 \\
MCAS Miramar & Marine Corps Air Station & 3 & - & 4 & - & 2 & 5 & 14 \\
MCAGCC 29 Palms & $\begin{array}{l}\text { Marine Corps Air Ground } \\
\text { Combat Center }\end{array}$ & 1 & - & - & - & - & - & 1 \\
Total & & 19 & - & 12 & - & 15 & 27 & 73 \\
\hline
\end{tabular}

\subsection{Vehicles Selected for Monitoring}

Seventy-three vehicles were included in the study at INL. Table A-1 in Appendix A presents an index of vehicles in tabular form, including vehicle make, model, year, and base. Selection of monitored vehicles was completed by U.S. Navy personnel. The objective of this selection was to determine viable $\mathrm{BEV}$ replacement candidates.

Appendix B provides a detailed summary analysis of each individual vehicle included in this study. The information from this analysis is presented by grouping the vehicles into their respective bases of operation. Because the base missions vary considerably, INL choose to group these mission areas and evaluate them separately. This will ensure that mission gaps are not created by recommended replacements.

\subsection{Data Validity}

INL data collection took place from August 1, 2014, through July 1, 2015. Vehicle data sheets (presented in Appendix B) detail the summary information plots and statistics related to each vehicle.

Five of the vehicles listed did not have any data collected in the analysis time period. One vehicle did not provide enough information to form a reliable recommendation. Table 4 shows this information by mission location.

Table 4. Base evaluations showing percent studied.

\begin{tabular}{lccc} 
Vehicle Mission & Study Vehicles & Total Reported & Percent Studied \\
CLB Barstow & 5 & 4 & $80.0 \%$ \\
CB Camp Pendleton & 53 & 50 & $94.3 \%$ \\
CAS Miramar & 14 & 13 & $92.9 \%$ \\
CAGCC 29 Palms & 1 & 1 & $100.0 \%$ \\
\hline
\end{tabular}




\subsection{MCLB Barstow Vehicle Pool Evaluation}

\subsubsection{Site Information}

At MCLB Barstow, data were collected from one mid-size sedan, one passenger van, and two small trucks.

Incorporation of BEVs into the pool mission is a definite possibility. Pool vehicles used for shorter trips qualify for BEV replacement, while other pool vehicle activities that are used for longer trips may require range beyond that of the available BEVs.

\subsubsection{Summary for Pool Vehicles}

Appendix B provides the vehicle data sheets for each of the pool vehicles monitored. This section aggregates data for all pool vehicles for MCLB Barstow. Table 5 summarizes pool vehicle travel during the study period for those days when the vehicle was driven. Vehicle use occurred primarily between 0800 and 2200 hours daily. The vehicles were driven 22,959 miles and logged 1,277 hours of operation. Vehicle 5B-G413964H did not provide data and was excluded from the summary and range analyses, because inclusion of the vehicle would skew the results in both cases.

Table 5. MCLB Barstow evaluation.

\section{Pool Summary}

$\begin{array}{lc}\text { Total Distance (miles) } & 22,959.2 \\ \text { Total Drive Time (hours) } & 1,277 \\ \text { Daily Average Trip Distance (miles) } & 36.0 \\ \text { Percent of Days Driven } & 49 \%\end{array}$

Table 6 summarizes how often a pool vehicle exceeded the low, medium, and high electric vehicle ranges. The BEV ranges specified for low, medium, and high-range BEVs represent the expected single-charge ranges of BEVs that are currently available.

Table 6. MCLB Barstow range analysis.

\begin{tabular}{lccc} 
& $\begin{array}{c}\text { Low Range, } \\
\text { Medium Range, } \\
\text { High Range }\end{array}$ & $\begin{array}{c}\text { Percentage of Days } \\
\text { Vehicle Exceeds } \\
\text { Electric Vehicle Range }\end{array}$ & $\begin{array}{c}\text { Number of Days Vehicle } \\
\text { Exceeds Electric } \\
\text { Vehicle Range }\end{array}$ \\
\hline 5B-294399 & 59 & $14 \%$ & 8 \\
& 63 & $9 \%$ & 5 \\
5B-G410512G & 67 & $9 \%$ & 5 \\
& 59 & $5 \%$ & 10 \\
5B-G410523G & 63 & $3 \%$ & 7 \\
& 67 & $3 \%$ & 6 \\
5B-G414199H & 59 & $8 \%$ & 20 \\
& 67 & $6 \%$ & 14 \\
& 59 & $20 \%$ & 12 \\
& 63 & $20 \%$ & 29 \\
\hline
\end{tabular}




\subsubsection{Individual Vehicle Observations Compared to 59, 63, and 67-Mile Range Limits}

1. Vehicle ID 5B-294399, Sedan

This vehicle was driven 56 days of the 334 days in the study period (16.7\%). The vehicle exceeded the 59-mile range limit on 8 days (14\%). The vehicle exceeded the 63-mile range limit on 5 days (9\%). The vehicle exceeded the 67-mile range limit on 5 days (9\%). As shown in the vehicle data sheet in Appendix B, the vehicle performed a moderate number of short trips per day, possibly allowing daytime charge events.

This mid-sized sedan may be replaced with a BEV, provided that another vehicle is available for the long-range driving this vehicle occasionally supported. Charging can be accomplished overnight or on days when the vehicle is not driven.

2. Vehicle ID 5B-G410512G, Passenger Van

This vehicle was driven 205 days of the 334 days in the study period (61.3\%). The vehicle exceeded the 59-mile range limit on 10 days or (5\%). The vehicle exceeded the 63-mile range limit on 7 days $(3 \%)$. The vehicle exceeded the 67 -mile range limit on 6 days $(3 \%)$. As shown in the vehicle data sheet in Appendix B, the vehicle performed a moderate number of short trips per day, possibly allowing daytime charge events.

This passenger van may be replaced with a BEV, provided that another vehicle is available for the long-range driving this vehicle occasionally supported. Charging can be accomplished overnight or on days when the vehicle is not driven.

3. Vehicle ID 5B-G410523G, Small Truck

This vehicle was driven 250 days of the 334 days in the study period (74.8\%). The vehicle exceeded the 59-mile range limit on 29 days $(8 \%)$. The vehicle exceeded the 63-mile range limit on 29 days $(6 \%)$. The vehicle exceeded the 67-mile range limit on 27 days $(5 \%)$. As shown in the vehicle data sheet in Appendix B, the vehicle performed a moderate number of short trips per day, possibly allowing daytime charge events.

This small truck may be replaced with a BEV, provided that another vehicle is available for the long-range driving this vehicle occasionally supported. Charging can be accomplished overnight or on days when the vehicle is not driven.

4. Vehicle ID 5B-G413964H, Minivan

No data were provided for this vehicle over the study period.

5. Vehicle ID 5B-G414199H, Small Truck

This vehicle was driven 143 days of the 334 days in the study period (42.8\%). The vehicle exceeded the 59-mile range limit on 27 days (20\%). The vehicle exceeded the 63-mile range limit on 27 days $(20 \%)$. The vehicle exceeded the 67-mile range limit on 27 days $(19 \%)$. As shown in the vehicle data sheet in Appendix B, the vehicle performed a moderate number of short trips per day, possibly allowing daytime charge events.

This small truck may be replaced with a BEV, provided that another vehicle is available for the long-range driving this vehicle occasionally supported. Charging can be accomplished overnight or on days when the vehicle is not driven. 


\subsection{MCB Camp Pendleton Vehicle Pool Evaluation}

\subsubsection{Site Information}

At MCB Camp Pendleton, data were collected from 13 mid-size sedans, 17 vans, nine small trucks, and 11 trucks for a total of 50 vehicles.

Incorporation of BEVs into the pool mission is a definite possibility. Pool vehicles used for shorter trips qualify for BEV replacement, while other pool vehicle activities used for longer trips may require range beyond that of available BEVs.

\subsubsection{Summary for Pool Vehicles}

Appendix B provides the vehicle data sheets for each of the pool vehicles monitored. This section aggregates data for all pool vehicles for MCB Camp Pendleton. Table 7 summarizes pool travel during the study period for those days when the vehicle was driven. Vehicle use occurred primarily between 0800 and 2200 hours daily. The vehicles were driven a total of 256,824 miles and logged 7,733 hours of operation. Vehicles 5L-G109773K, 5L-G414871K, 5L-G414872K, and 5L-G416051H did not provide a sufficient amount of data; therefore, they were excluded in the summary and range analyses, because inclusion of the vehicles would skew the results in both cases.

Table 7. MCB Camp Pendleton evaluation.

\section{Pool Summary}

Total Distance (miles) 256,823.5

Total Drive Time (hours) $\quad 7,733$

Daily Average Trip Distance (miles) 33.6

Percent of Days Driven $\quad 43.2 \%$

Table 8 summarizes how often a pool vehicle exceeds the low, medium, and high electric vehicle ranges. The BEV ranges specified for low, medium, and high-range BEVs represent the expected single-charge ranges of BEVs that are currently available.

Table 8. MCB Camp Pendleton range.

\begin{tabular}{lccc} 
& $\begin{array}{c}\text { Low Range, } \\
\text { Medium Range, } \\
\text { High Range }\end{array}$ & $\begin{array}{c}\text { EV Range } \\
\text { Percentage of Days } \\
\text { Vehicle Exceeds } \\
\text { Electric Vehicle Range }\end{array}$ & $\begin{array}{c}\text { Number of Days } \\
\text { Vehicle Exceeds } \\
\text { Electric Vehicle Range }\end{array}$ \\
\hline 5L-294266 & 59 & $9 \%$ & 10 \\
& 63 & $9 \%$ & 10 \\
5L-294267 & 67 & $9 \%$ & 10 \\
& 59 & $5 \%$ & 5 \\
5 5L-294274 & 63 & $3 \%$ & 3 \\
& 67 & $3 \%$ & 3 \\
5 & 59 & $5 \%$ & 7 \\
5L-294277 & 63 & $3 \%$ & 5 \\
& 67 & $6 \%$ & 3 \\
5L-G106206H & 59 & $6 \%$ & 4 \\
& 63 & $5 \%$ & 3 \\
& 67 & $22 \%$ & 37 \\
\hline
\end{tabular}




\begin{tabular}{|c|c|c|c|}
\hline & $\begin{array}{l}\text { Low Range, } \\
\text { Medium Range, } \\
\text { High Range }\end{array}$ & $\begin{array}{l}\text { EV Range } \\
\text { Percentage of Days } \\
\text { Vehicle Exceeds } \\
\text { Electric Vehicle Range }\end{array}$ & $\begin{array}{c}\text { Number of Days } \\
\text { Vehicle Exceeds } \\
\text { Electric Vehicle Range }\end{array}$ \\
\hline \multirow{3}{*}{ 5L-G106208H } & 59 & $36 \%$ & 32 \\
\hline & 63 & $34 \%$ & 31 \\
\hline & 67 & $33 \%$ & 30 \\
\hline \multirow[t]{3}{*}{ 5L-G106501H } & 59 & $6 \%$ & 6 \\
\hline & 63 & $6 \%$ & 6 \\
\hline & 67 & $6 \%$ & 6 \\
\hline \multirow[t]{3}{*}{ 5L-G107279L } & 59 & $17 \%$ & 34 \\
\hline & 63 & $15 \%$ & 30 \\
\hline & 67 & $13 \%$ & 25 \\
\hline \multirow[t]{3}{*}{ 5L-G109766K } & 59 & $5 \%$ & 9 \\
\hline & 63 & $5 \%$ & 9 \\
\hline & 67 & $5 \%$ & 9 \\
\hline \multirow[t]{3}{*}{ 5L-G109768K } & 59 & $13 \%$ & 22 \\
\hline & 63 & $12 \%$ & 21 \\
\hline & 67 & $12 \%$ & 20 \\
\hline \multirow[t]{3}{*}{ 5L-G109769K } & 59 & $12 \%$ & 28 \\
\hline & 63 & $10 \%$ & 24 \\
\hline & 67 & $9 \%$ & 21 \\
\hline \multirow[t]{3}{*}{ 5L-G109771K } & 59 & $17 \%$ & 10 \\
\hline & 63 & $17 \%$ & 10 \\
\hline & 67 & $13 \%$ & 8 \\
\hline \multirow[t]{3}{*}{ 5L-G136271L } & 59 & $1 \%$ & 1 \\
\hline & 63 & $1 \%$ & 1 \\
\hline & 67 & $1 \%$ & 1 \\
\hline \multirow{3}{*}{ 5L-G410487G } & 59 & $15 \%$ & 19 \\
\hline & 63 & $12 \%$ & 16 \\
\hline & 67 & $10 \%$ & 13 \\
\hline \multirow[t]{3}{*}{ 5L-G410489G } & 59 & $23 \%$ & 40 \\
\hline & 63 & $20 \%$ & 34 \\
\hline & 67 & $18 \%$ & 31 \\
\hline \multirow{3}{*}{ 5L-G410514G } & 59 & $38 \%$ & 10 \\
\hline & 63 & $35 \%$ & 9 \\
\hline & 67 & $35 \%$ & 9 \\
\hline \multirow[t]{3}{*}{ 5L-G411436F } & 59 & $2 \%$ & 4 \\
\hline & 63 & $2 \%$ & 4 \\
\hline & 67 & $2 \%$ & 4 \\
\hline \multirow[t]{3}{*}{ 5L-G411447F } & 59 & $35 \%$ & 33 \\
\hline & 63 & $33 \%$ & 31 \\
\hline & 67 & $30 \%$ & 28 \\
\hline \multirow[t]{3}{*}{ 5L-G411466F } & 59 & $9 \%$ & 13 \\
\hline & 63 & $7 \%$ & 10 \\
\hline & 67 & $6 \%$ & 9 \\
\hline \multirow{3}{*}{ 5L-G411467F } & 59 & $2 \%$ & 3 \\
\hline & 63 & $1 \%$ & 2 \\
\hline & 67 & $1 \%$ & 2 \\
\hline 5L-G414389H & 59 & $19 \%$ & 36 \\
\hline
\end{tabular}




\begin{tabular}{|c|c|c|c|}
\hline \multicolumn{4}{|c|}{ EV Range } \\
\hline & $\begin{array}{l}\text { Low Range, } \\
\text { Medium Range, } \\
\text { High Range }\end{array}$ & $\begin{array}{c}\text { Percentage of Days } \\
\text { Vehicle Exceeds } \\
\text { Electric Vehicle Range }\end{array}$ & $\begin{array}{c}\text { Number of Days } \\
\text { Vehicle Exceeds } \\
\text { Electric Vehicle Range }\end{array}$ \\
\hline \multirow{4}{*}{ 5L-G414405H } & 63 & $14 \%$ & 27 \\
\hline & 67 & $12 \%$ & 24 \\
\hline & 59 & $4 \%$ & 7 \\
\hline & 63 & $3 \%$ & 5 \\
\hline \multirow{4}{*}{ 5L-G414428H } & 67 & $3 \%$ & 5 \\
\hline & 59 & $12 \%$ & 20 \\
\hline & 63 & $12 \%$ & 20 \\
\hline & 67 & $11 \%$ & 18 \\
\hline \multirow[t]{3}{*}{ 5L-G414430H } & 59 & $28 \%$ & 58 \\
\hline & 63 & $27 \%$ & 55 \\
\hline & 67 & $25 \%$ & 52 \\
\hline \multirow[t]{3}{*}{ 5L-G414438H } & 59 & $18 \%$ & 15 \\
\hline & 63 & $18 \%$ & 15 \\
\hline & 67 & $18 \%$ & 15 \\
\hline \multirow[t]{3}{*}{ 5L-G414440H } & 59 & $9 \%$ & 18 \\
\hline & 63 & $7 \%$ & 13 \\
\hline & 67 & $6 \%$ & 11 \\
\hline \multirow{3}{*}{ 5L-G414488L } & 59 & $8 \%$ & 16 \\
\hline & 63 & $6 \%$ & 12 \\
\hline & 67 & $5 \%$ & 10 \\
\hline \multirow[t]{3}{*}{ 5L-G414500L } & 59 & $21 \%$ & 13 \\
\hline & 63 & $21 \%$ & 13 \\
\hline & 67 & $20 \%$ & 12 \\
\hline \multirow{3}{*}{ 5L-G414511L } & 59 & $15 \%$ & 29 \\
\hline & 63 & $13 \%$ & 25 \\
\hline & 67 & $11 \%$ & 21 \\
\hline \multirow[t]{3}{*}{ 5L-G414513L } & 59 & $2 \%$ & 3 \\
\hline & 63 & $2 \%$ & 3 \\
\hline & 67 & $1 \%$ & 1 \\
\hline \multirow[t]{3}{*}{ 5L-G414867K } & 59 & $1 \%$ & 2 \\
\hline & 63 & $1 \%$ & 1 \\
\hline & 67 & $1 \%$ & 1 \\
\hline \multirow[t]{3}{*}{ 5L-G414868K } & 59 & $11 \%$ & 12 \\
\hline & 63 & $11 \%$ & 12 \\
\hline & 67 & $11 \%$ & 12 \\
\hline \multirow{3}{*}{ 5L-G414869K } & 59 & $9 \%$ & 14 \\
\hline & 63 & $6 \%$ & 10 \\
\hline & 67 & $5 \%$ & 8 \\
\hline \multirow[t]{3}{*}{ 5L-G421041D } & 59 & $0 \%$ & 0 \\
\hline & 63 & $0 \%$ & 0 \\
\hline & 67 & $0 \%$ & 0 \\
\hline \multirow[t]{3}{*}{ 5L-G421775L } & 59 & $8 \%$ & 18 \\
\hline & 63 & $6 \%$ & 13 \\
\hline & 67 & $5 \%$ & 11 \\
\hline \multirow[t]{2}{*}{ 5L-G421920L } & 59 & $36 \%$ & 96 \\
\hline & 63 & $32 \%$ & 85 \\
\hline
\end{tabular}




\begin{tabular}{|c|c|c|c|}
\hline \multicolumn{4}{|c|}{ EV Range } \\
\hline & $\begin{array}{l}\text { Low Range, } \\
\text { Medium Range, } \\
\text { High Range }\end{array}$ & $\begin{array}{c}\text { Percentage of Days } \\
\text { Vehicle Exceeds } \\
\text { Electric Vehicle Range }\end{array}$ & $\begin{array}{c}\text { Number of Days } \\
\text { Vehicle Exceeds } \\
\text { Electric Vehicle Range }\end{array}$ \\
\hline \multirow{4}{*}{ 5L-G421922L } & 67 & $29 \%$ & 78 \\
\hline & 59 & $18 \%$ & 11 \\
\hline & 63 & $13 \%$ & 8 \\
\hline & 67 & $13 \%$ & 8 \\
\hline \multirow[t]{3}{*}{ 5L-G422169G } & 59 & $45 \%$ & 57 \\
\hline & 63 & $44 \%$ & 56 \\
\hline & 67 & $44 \%$ & 55 \\
\hline \multirow[t]{3}{*}{ 5L-G422206H } & 59 & $28 \%$ & 34 \\
\hline & 63 & $26 \%$ & 32 \\
\hline & 67 & $25 \%$ & 31 \\
\hline \multirow[t]{3}{*}{ 5L-G422215H } & 59 & $49 \%$ & 125 \\
\hline & 63 & $42 \%$ & 107 \\
\hline & 67 & $40 \%$ & 102 \\
\hline \multirow[t]{3}{*}{ 5L-G422227H } & 59 & $22 \%$ & 33 \\
\hline & 63 & $21 \%$ & 31 \\
\hline & 67 & $21 \%$ & 31 \\
\hline \multirow[t]{3}{*}{ 5L-G422233H } & 59 & $5 \%$ & 8 \\
\hline & 63 & $3 \%$ & 5 \\
\hline & 67 & $3 \%$ & 4 \\
\hline \multirow[t]{3}{*}{ 5L-G422235H } & 59 & $8 \%$ & 13 \\
\hline & 63 & $8 \%$ & 13 \\
\hline & 67 & $7 \%$ & 11 \\
\hline \multirow[t]{3}{*}{ 5L-G422467K } & 59 & $1 \%$ & 2 \\
\hline & 63 & $0 \%$ & 1 \\
\hline & 67 & $0 \%$ & 0 \\
\hline \multirow{3}{*}{ 5L-G422482K } & 59 & $27 \%$ & 41 \\
\hline & 63 & $27 \%$ & 40 \\
\hline & 67 & $25 \%$ & 38 \\
\hline \multirow[t]{3}{*}{ 5L-G422486K } & 59 & $7 \%$ & 13 \\
\hline & 63 & $6 \%$ & 11 \\
\hline & 67 & $5 \%$ & 10 \\
\hline \multirow{3}{*}{ 5L-G422785H } & 59 & $12 \%$ & 25 \\
\hline & 63 & $11 \%$ & 23 \\
\hline & 67 & $10 \%$ & 20 \\
\hline \multirow[t]{3}{*}{ 5L-G422884H } & 59 & $1 \%$ & 2 \\
\hline & 63 & $1 \%$ & 1 \\
\hline & 67 & $1 \%$ & 1 \\
\hline \multirow[t]{3}{*}{ 5L-G422886H } & 59 & $5 \%$ & 6 \\
\hline & 63 & $4 \%$ & 4 \\
\hline & 67 & $2 \%$ & 2 \\
\hline
\end{tabular}

\subsubsection{Vehicle Observations Compared to 63-Mile Range Limit}

Because this motor pool is much larger than the Barstow motor pool, analysis was performed by assigning the vehicles into groups rather than by a vehicle-by-vehicle analysis, including the following groups: 
- Replacement recommended no impact

- Replacement impact of 1 to 10 days (replacement possible; however, either additional study is required or the stakeholder can make a decision based on need or future vehicle utilization)

- Replacement impact greater than 10 days

- No recommendation provided (due to insufficient data).

Because the midpoint 63-mile range compares reasonably to real world results, it provided a useful basis of comparison across the vehicle groups.

4.5.3.1 Vehicle Replacement No Impact. Analysis of these vehicles indicates that their daily usage may or may not exceed the 63-mile range limit. These vehicles may be replaced by BEVs, with the assumption that the motor pool vehicles not recommended for replacement will be able to support the rare days when these vehicles were used to support long-distance driving. No vehicles meet a no impact condition.

4.5.3.2 Vehicle Replacement Impact of 1 to 10 Days. Analysis of these vehicles indicates that their daily usage may or may not exceed the 63-mile range limit, but it occurs for a higher number of trips and the frequency is enough that it warrants cautious consideration or additional study prior to replacing the vehicle with a BEV. Based on the statistics, it appears that operational risk or impact could be presented by going forward with BEV replacement. The following 22 vehicles have an impact of 1 to 10 days:

- $\quad 5 \mathrm{~L}-294266$

- $\quad 5 \mathrm{~L}-294267$

- $\quad 5 \mathrm{~L}-294274$

- $\quad 5 \mathrm{~L}-294277$

- $5 \mathrm{~L}-\mathrm{G} 106501 \mathrm{H}$

- 5L-G109766K

- $5 \mathrm{~L}-\mathrm{G} 109771 \mathrm{~K}$

- 5L-G136271L
- 5L-G410514G

- 5L-G411436F

- 5L-G411466F

- 5L-G411467F

- $5 \mathrm{~L}-\mathrm{G} 414405 \mathrm{H}$

- 5L-G414513L

- $5 \mathrm{~L}-\mathrm{G} 414867 \mathrm{~K}$

- $5 \mathrm{~L}-\mathrm{G} 414869 \mathrm{~K}$
- 5L-G421041D

- 5L-G421922L

- $5 \mathrm{~L}-\mathrm{G} 422233 \mathrm{H}$

- $5 \mathrm{~L}-\mathrm{G} 422467 \mathrm{~K}$

- $5 \mathrm{~L}-\mathrm{G} 422884 \mathrm{H}$

- $5 \mathrm{~L}-\mathrm{G} 422886 \mathrm{H}$

4.5.3.3 Vehicle Replacement Impact of Greater Than 10 Days. Analysis of these vehicles indicates that their daily usage frequently exceeds the 63-mile range limit and their high utilization (i.e., number of trips per day) makes supplemental charging during daytime operations difficult to meet the mission objectives. Consideration of the transportation of cargo and personnel also factors into this recommendation. Based on the statistics, it appears that replacing these vehicles would present high operational risk or impact. The following 27 vehicles have an impact of greater than 10 days. Replacement with a BEVs represents a significant risk to the operational mission:

- 5L-G106206H

- $5 \mathrm{~L}-\mathrm{G} 106208 \mathrm{H}$

- 5L-G107279L

- 5L-G109768K

- $5 \mathrm{~L}-\mathrm{G} 109769 \mathrm{~K}$

- $5 \mathrm{~L}-\mathrm{G} 410487 \mathrm{G}$
- 5L-G410489G

- 5L-G411447F

- 5L-G414389H

- 5L-G414428H

- 5L-G414430H

- 5L-G414438H
- $5 \mathrm{~L}-\mathrm{G} 414440 \mathrm{H}$

- 5L-G414488L

- 5L-G414500L

- 5L-G414511L

- $5 \mathrm{~L}-\mathrm{G} 414868 \mathrm{~K}$

- 5L-G421775L 
- $5 \mathrm{~L}-\mathrm{G} 421920 \mathrm{~L}$

- $5 \mathrm{~L}-\mathrm{G} 422169 \mathrm{G}$

- $5 \mathrm{~L}-\mathrm{G} 422206 \mathrm{H}$
- $5 \mathrm{~L}-\mathrm{G} 422215 \mathrm{H}$

- $5 \mathrm{~L}-\mathrm{G} 422227 \mathrm{H}$

- $5 \mathrm{~L}-\mathrm{G} 422235 \mathrm{H}$
- $5 \mathrm{~L}-\mathrm{G} 422482 \mathrm{~K}$

- $5 \mathrm{~L}-\mathrm{G} 422486 \mathrm{~K}$

- $5 \mathrm{~L}-\mathrm{G} 422785 \mathrm{H}$

4.5.3.4 No Recommendation Provided. Not enough data were available to provide a recommendation for replacement of the following four vehicles. Additional study would be required to determine if these vehicles can be replaced by BEVs. These vehicles are as follows:

- $5 \mathrm{~L}-\mathrm{G} 109773 \mathrm{~K}$

- $5 \mathrm{~L}-\mathrm{G} 414871 \mathrm{~K}$

- $5 \mathrm{~L}-\mathrm{G} 414872 \mathrm{~K}$

- $5 \mathrm{~L}-\mathrm{G} 416051 \mathrm{H}$

\subsection{MCAS Miramar Vehicle Pool Evaluation}

\subsubsection{Site Information}

At MCAS Miramar, data were collected from three mid-size sedans, five vans, two small trucks, and three full size trucks for a total of 13 vehicles.

Incorporation of BEVs into the pool mission is a definite possibility. Pool vehicles used for shorter trips qualify for BEV replacement, while other pool vehicle activities that are used for longer trips may require range beyond that of available BEVs.

\subsubsection{Summary for Pool Vehicles}

Appendix B provides the vehicle data sheets for each of the pool vehicles monitored. This section aggregates data for all pool vehicles for MCAS Miramar. Table 9 summarizes pool travel during the study period for those days when the vehicle was driven. Vehicle use occurred primarily between 0800 and 2200 hours daily. The vehicles were driven 54,072 miles and logged 1603 hours of operation. Vehicle 5M-414515L did not provide a sufficient amount of data; therefore, it was excluded in the summary and range analyses, because inclusion of the vehicle would skew the results in both cases.

Table 9. MCAS Miramar evaluation.

\begin{tabular}{lc}
\multicolumn{2}{c}{ Pool Summary } \\
Total Distance (miles) & $54,071.8$ \\
Total Drive Time (hours) & 1,603 \\
Daily Average Trip Distance (miles) & 27.3 \\
Percent of Days Driven & $42.4 \%$ \\
\hline
\end{tabular}

Table 10 summarizes how often a pool vehicle exceeds the low, medium, and high electric vehicle ranges. The BEV ranges specified for low, medium, and high-range BEVs represent the expected single-charge ranges of BEVs that are currently available.

Table 10. MCAS Miramar range analysis.

\begin{tabular}{cccc} 
& $\begin{array}{c}\text { Low Range, } \\
\text { Medium Range, } \\
\text { High Range }\end{array}$ & $\begin{array}{c}\text { Percentage of Days } \\
\text { Vehicle Exceeds } \\
\text { Electric Vehicle Range }\end{array}$ & $\begin{array}{c}\text { Number of Days Vehicle } \\
\text { Exceeds Electric } \\
\text { Vehicle Range }\end{array}$ \\
\hline 5M-G106209H & 59 & $18 \%$ & 31 \\
& 63 & $18 \%$ & 31 \\
& 67 & $16 \%$ & 28 \\
\hline
\end{tabular}




\section{EV Range}

Low Range,

Medium Range,

Percentage of Days

Number of Days Vehicle High Range

Vehicle Exceeds Exceeds Electric Electric Vehicle Range Vehicle Range

\begin{tabular}{|c|c|c|c|}
\hline \multirow[t]{3}{*}{ 5M-G106212H } & 59 & $6 \%$ & 9 \\
\hline & 63 & $6 \%$ & 9 \\
\hline & 67 & $6 \%$ & 9 \\
\hline \multirow[t]{3}{*}{ 5M-G136272L } & 59 & $16 \%$ & 21 \\
\hline & 63 & $15 \%$ & 20 \\
\hline & 67 & $13 \%$ & 18 \\
\hline \multirow[t]{3}{*}{ 5M-G414171H } & 59 & $3 \%$ & 3 \\
\hline & 63 & $3 \%$ & 3 \\
\hline & 67 & $3 \%$ & 3 \\
\hline \multirow[t]{3}{*}{ 5M-G414446H } & 59 & $5 \%$ & 8 \\
\hline & 63 & $2 \%$ & 5 \\
\hline & 67 & $1 \%$ & 3 \\
\hline \multirow[t]{3}{*}{ 5M-G414516L } & 59 & $5 \%$ & 7 \\
\hline & 63 & $5 \%$ & 7 \\
\hline & 67 & $4 \%$ & 6 \\
\hline \multirow[t]{3}{*}{ 5M-G414859K } & 59 & $46 \%$ & 39 \\
\hline & 63 & $44 \%$ & 37 \\
\hline & 67 & $41 \%$ & 35 \\
\hline \multirow[t]{3}{*}{ 5M-G414860K } & 59 & $8 \%$ & 12 \\
\hline & 63 & $8 \%$ & 12 \\
\hline & 67 & $7 \%$ & 11 \\
\hline \multirow[t]{3}{*}{ 5M-G422183G } & 59 & $43 \%$ & 39 \\
\hline & 63 & $41 \%$ & 37 \\
\hline & 67 & $40 \%$ & 36 \\
\hline \multirow[t]{3}{*}{$5 \mathrm{M}-\mathrm{G} 422205 \mathrm{H}$} & 59 & $29 \%$ & 49 \\
\hline & 63 & $28 \%$ & 48 \\
\hline & 67 & $28 \%$ & 47 \\
\hline \multirow[t]{3}{*}{ 5M-G422890H } & 59 & $17 \%$ & 35 \\
\hline & 63 & $17 \%$ & 35 \\
\hline & 67 & $16 \%$ & 34 \\
\hline \multirow[t]{3}{*}{ 5M-G422891H } & 59 & $6 \%$ & 8 \\
\hline & 63 & $5 \%$ & 7 \\
\hline & 67 & $4 \%$ & 6 \\
\hline \multirow[t]{3}{*}{ 5M-G423185H } & 59 & $5 \%$ & 9 \\
\hline & 63 & $5 \%$ & 9 \\
\hline & 67 & $5 \%$ & 9 \\
\hline
\end{tabular}




\subsubsection{Vehicle Observations Compared to a 63-Mile Range Limit}

Analysis of the vehicles required the vehicles to be aggregated into the following groups due to the size of the motor pool:

- Replacement recommended no impact

- Replacement impact of 1 to 10 days (however, either additional study is required or the stakeholder can make a decision based on need or future vehicle utilization)

- Replacement impact greater than 10 days

- No recommendation provided (due to no data available or insufficient data for a result).

4.6.3.1 Vehicle Replacement No Impact. Analysis of these vehicles indicates that their daily usage may or may not exceed the 63-mile range limit. These vehicles may be replaced by BEVs, with the assumption that motor pool vehicles not recommended for replacement will be able to support the rare days when these vehicles were used to support long-distance driving. No vehicles meet a no impact condition.

4.6.3.2 Vehicle Replacement Impact of 1 to 10 Days. Analysis of these vehicles indicates that their daily usage may or may not exceed the 63-mile range limit, but it occurs for a higher number of trips and the frequency is enough that it warrants cautious consideration or additional study prior to replacing the vehicle with a BEV. Based on the statistics, it appears that operational risk or impact could be presented by going forward with BEV replacement. The following six vehicles have an impact of 1 to 10 days:

- $5 \mathrm{M}-\mathrm{G} 106212 \mathrm{H}$

- $5 \mathrm{M}-\mathrm{G} 414171 \mathrm{H}$
- $5 \mathrm{M}-\mathrm{G} 414446 \mathrm{H}$

- $5 \mathrm{M}-\mathrm{G} 414516 \mathrm{~L}$
- $5 \mathrm{M}-\mathrm{G} 422891 \mathrm{H}$

- $5 \mathrm{M}-\mathrm{G} 423185 \mathrm{H}$

4.6.3.3 Vehicle Replacement Impact of Greater Than 10 Days. Analysis of these vehicles indicates that their daily usage frequently exceeds the 63-mile range limit and their high utilization (i.e., number of trips per day) makes supplemental charging during daytime operations difficult to meet the mission objectives. Consideration of the transportation of cargo and personnel also factors into this recommendation. Based on the statistics, it appears that replacing these vehicles would present high operational risk or impact. The following seven vehicles have an impact of greater than 10 days. Replacement with a BEVs represents a significant risk to the operational mission.

- $5 \mathrm{M}-\mathrm{G} 106209 \mathrm{H}$

- $5 \mathrm{M}-\mathrm{G} 136272 \mathrm{~L}$

- $5 \mathrm{M}-\mathrm{G} 422205 \mathrm{H}$
- $5 \mathrm{M}-\mathrm{G} 414860 \mathrm{~K}$

- $5 \mathrm{M}-\mathrm{G} 422890 \mathrm{H}$
- $5 \mathrm{M}-\mathrm{G} 414859 \mathrm{~K}$

- $5 \mathrm{M}-\mathrm{G} 422183 \mathrm{G}$

4.6.3.4 No Recommendation Provided. Not enough data were available to provide a recommendation for replacement of one vehicle. Additional study would be required to determine if this vehicle can be replaced by a BEV. The vehicle is $5 \mathrm{M}-\mathrm{G} 414515 \mathrm{~L}$.

\subsection{MCAGCC 29 Palms Vehicle Pool Evaluation}

\subsubsection{Site Information}

At MCAGCC 29 Palms, data were collected from only a single mid-size sedan.

Incorporation of BEVs into the pool mission is a definite possibility. Pool vehicles used for shorter trips qualify for BEV replacement, while other pool vehicle activities used for longer trips may require range beyond that of available BEVs. 


\subsubsection{Summary for Pool Vehicle}

Appendix B provides the vehicle data sheet for the pool vehicle monitored. This section aggregates data for the MCAGCC 29 Palms pool vehicle. Table 11 summarizes pool vehicle travel during the study period for those days when the vehicle was driven. Vehicle use occurred primarily between 0800 and 2200 hours daily. The vehicle was driven 5,688 miles and logged 279 hours of operation.

Table 11. MCAGCC 29 Palms evaluation.

\section{Pool Summary}

Total Distance (miles)

$5,688.2$

Total Drive Time (hours)

279

Daily Average Trip Distance (miles)

18.5

Percent of Days Driven

$91.9 \%$

Table 12 summarizes how often a pool vehicle exceeds the low, medium, and high electric vehicle ranges. The BEV ranges specified for low, medium, and high-range BEVs represent the expected single-charge ranges of BEVs that are currently available.

Table 12. MCAGCC 29 Palms range analysis.

\begin{tabular}{|c|c|c|c|}
\hline \multicolumn{4}{|c|}{ EV Range } \\
\hline & $\begin{array}{c}\text { Low Range, } \\
\text { Medium Range, } \\
\text { High Range }\end{array}$ & $\begin{array}{l}\text { Percentage of Days } \\
\text { Vehicle Exceeds } \\
\text { Electric Vehicle Range }\end{array}$ & $\begin{array}{c}\text { Number of Days } \\
\text { Vehicle Exceeds } \\
\text { Electric Vehicle Range }\end{array}$ \\
\hline \multirow[t]{3}{*}{ 5P-G130225A } & 59 & $2 \%$ & 6 \\
\hline & 63 & $2 \%$ & 5 \\
\hline & 67 & $1 \%$ & 4 \\
\hline
\end{tabular}

\subsubsection{Individual Vehicle Observation(s) Compared to 59, 63, and 67-Mile Range Limits}

1. Vehicle ID 5P-G130225A, Sedan

This vehicle was driven 274 days of the 334 days in the study period $(82.0 \%)$. The vehicle exceeded the 59-mile range limit on 6 days (2\%). The vehicle exceeded the 63 -mile range limit on 5 days $(2 \%)$. The vehicle exceeded the 67-mile range limit on 4 days (1\%). As shown on the vehicle data sheet in Appendix B, the vehicle performed a moderate number of short trips per day, possibly allowing for daytime charge events.

This compact sedan vehicle may be replaced with a BEV, provided that another vehicle is available for the long-range driving this vehicle occasionally supported. Charging can be accomplished overnight or on days when the vehicle is not driven.

\section{OBSERVATIONS}

INL appreciates the opportunity to present the results of this evaluation for these 73 specific vehicles. Observations for possible follow-up actions include the following:

Infrastructure Planning: In conjunction with the replacement plan, evaluation of U.S. Navy sites for placement of PEV charging infrastructure could be beneficial. INL has significant experience in this area and such plans will consider fleet vehicle charging needs and the convenience that charging infrastructure provides employees and visitors. This planning also considers the existing facility electrical distribution 
system. Vehicle home base considerations factor into the ratio of PEVs to electric vehicle supply equipment units to maintain all vehicles at operational readiness.

Additional Investigations: A more thorough examination of the quantities and types of fleet vehicles within each usage category may be beneficial to quantify additional fleet replacements by PEVs. In particular, an investigation of vehicles that essentially stay within one military installation and those that regularly transit between bases may be helpful in providing details of long-duration trips. Also, a detailed analysis that focuses more on daily use of the vehicles (such as vehicle parking locations and durations, frequency of long-trip distances by vehicle, and time-of-use metrics) to provide a finer view for future replacement strategies may be beneficial. 


\section{Appendix A \\ U.S. Navy Vehicle Index}

Table A-1. INL vehicle index.

\begin{tabular}{|c|c|c|c|c|c|c|}
\hline \multicolumn{7}{|c|}{ Vehicle Index } \\
\hline $\begin{array}{l}\text { Vehicle } \\
\text { No. }\end{array}$ & Vehicle Label & Make & Model & Year & Type & Base \\
\hline 1 & 5B-294399 & Ford & Fusion & 2010 & Sedan & MCLB Barstow \\
\hline 2 & 5B-G410512G & Chevrolet & Uplander LS & 2008 & Van & MCLB Barstow \\
\hline 3 & 5B-G410523G & Chevrolet & Colorado & 2008 & Small pickup & MCLB Barstow \\
\hline 4 & 5B-G413964H & Dodge & Caravan & 2009 & Van & MCLB Barstow \\
\hline 5 & 5B-G414199H & Dodge & Dakota & 2009 & Small pickup & MCLB Barstow \\
\hline 6 & 5L-294266 & Chevy & Malibu & 2009 & Sedan & MCB Camp Pendleton \\
\hline 7 & 5L-294267 & Chevy & Malibu & 2009 & Sedan & MCB Camp Pendleton \\
\hline 8 & 5L-294274 & Chevy & Malibu & 2009 & Sedan & MCB Camp Pendleton \\
\hline 9 & 5L-294277 & Chevy & Malibu & 2009 & Sedan & MCB Camp Pendleton \\
\hline 10 & $5 \mathrm{~L}-\mathrm{G} 106206 \mathrm{H}$ & Pontiac & G6 & 2009 & Sedan & MCB Camp Pendleton \\
\hline 11 & $5 \mathrm{~L}-\mathrm{G} 106208 \mathrm{H}$ & Pontiac & G6 & 2009 & Sedan & MCB Camp Pendleton \\
\hline 12 & 5L-G106501H & Pontiac & G6 & 2009 & Sedan & MCB Camp Pendleton \\
\hline 13 & 5L-G107279L & Chevy & Malibu & 2011 & Sedan & MCB Camp Pendleton \\
\hline 14 & 5L-G109766K & Ford & Fusion Hybrid & 2011 & Sedan & MCB Camp Pendleton \\
\hline 15 & 5L-G109768K & Ford & Fusion Hybrid & 2011 & Sedan & MCB Camp Pendleton \\
\hline 16 & 5L-G109769K & Ford & Fusion Hybrid & 2011 & Sedan & MCB Camp Pendleton \\
\hline 17 & 5L-G109771K & Ford & Fusion Hybrid & 2011 & Sedan & MCB Camp Pendleton \\
\hline 18 & 5L-G109773K & Ford & Fusion Hybrid & 2011 & Sedan & MCB Camp Pendleton \\
\hline 19 & 5L-G136271L & Ford & Focus & 2012 & Sedan & MCB Camp Pendleton \\
\hline 20 & 5L-G410487G & Chevrolet & Uplander LS & 2008 & Van & MCB Camp Pendleton \\
\hline 21 & 5L-G410489G & Chevrolet & Uplander LS & 2008 & Van & MCB Camp Pendleton \\
\hline 22 & 5L-G410514G & Chevrolet & Uplander LS & 2008 & Van & MCB Camp Pendleton \\
\hline 23 & 5L-G411436F & Chevrolet & Uplander & 2007 & Van & MCB Camp Pendleton \\
\hline 24 & $5 \mathrm{~L}-\mathrm{G} 411447 \mathrm{~F}$ & Chevrolet & Uplander & 2007 & Van & MCB Camp Pendleton \\
\hline 25 & 5L-G411466F & Chevrolet & Uplander & 2007 & Van & MCB Camp Pendleton \\
\hline 26 & 5L-G411467F & Chevrolet & Uplander & 2007 & Van & MCB Camp Pendleton \\
\hline 27 & 5L-G414389H & Dodge & Caravan & 2009 & Van & MCB Camp Pendleton \\
\hline 28 & $5 \mathrm{~L}-\mathrm{G} 414405 \mathrm{H}$ & Dodge & Caravan & 2009 & Van & MCB Camp Pendleton \\
\hline 29 & $5 \mathrm{~L}-\mathrm{G} 414428 \mathrm{H}$ & Dodge & Dakota SXT & 2009 & Small pickup & MCB Camp Pendleton \\
\hline 30 & $5 \mathrm{~L}-\mathrm{G} 414430 \mathrm{H}$ & Dodge & Dakota SXT & 2009 & Small pickup & MCB Camp Pendleton \\
\hline 31 & 5L-G414438H & Dodge & Dakota & 2009 & Small pickup & MCB Camp Pendleton \\
\hline 32 & $5 \mathrm{~L}-\mathrm{G} 414440 \mathrm{H}$ & Dodge & Dakota & 2009 & Small pickup & MCB Camp Pendleton \\
\hline 33 & 5L-G414488L & Dodge & Grand Caravan & 2011 & Van & MCB Camp Pendleton \\
\hline 34 & 5L-G414500L & Dodge & Grand Caravan & 2011 & Van & MCB Camp Pendleton \\
\hline
\end{tabular}




\begin{tabular}{|c|c|c|c|c|c|c|}
\hline \multirow[b]{2}{*}{$\begin{array}{c}\text { Vehicle } \\
\text { No. }\end{array}$} & \multicolumn{5}{|c|}{ Vehicle Index } & \multirow[b]{2}{*}{ Base } \\
\hline & Vehicle Label & Make & Model & Year & Type & \\
\hline 35 & 5L-G414511L & Ram & Dakota & 2011 & Small pickup & MCB Camp Pendleton \\
\hline 36 & 5L-G414513L & Ram & Dakota & 2011 & Small pickup & MCB Camp Pendleton \\
\hline 37 & 5L-G414867K & Ford & Ranger & 2010 & Small pickup & MCB Camp Pendleton \\
\hline 38 & 5L-G414868K & Ford & Ranger & 2010 & Small pickup & MCB Camp Pendleton \\
\hline 39 & $5 \mathrm{~L}-\mathrm{G} 414869 \mathrm{~K}$ & Ford & Ranger & 2010 & Small pickup & MCB Camp Pendleton \\
\hline 40 & $5 \mathrm{~L}-\mathrm{G} 414871 \mathrm{~K}$ & Dodge & Grand Caravan & 2010 & Van & MCB Camp Pendleton \\
\hline 41 & 5L-G414872K & Dodge & Grand Caravan & 2010 & Van & MCB Camp Pendleton \\
\hline 42 & 5L-G416051H & Dodge & Grand Caravan & 2009 & Van & MCB Camp Pendleton \\
\hline 43 & 5L-G421041D & Chevrolet & Silverado & 2006 & Pickup & MCB Camp Pendleton \\
\hline 44 & 5L-G421775L & Ford & $\mathrm{F}-150$ & 2011 & Pickup & MCB Camp Pendleton \\
\hline 45 & 5L-G421920L & Ford & F-150 & 2011 & Pickup & MCB Camp Pendleton \\
\hline 46 & 5L-G421922L & Ford & F-150 & 2011 & Pickup & MCB Camp Pendleton \\
\hline 47 & 5L-G422169G & Chevrolet & Express & 2008 & Van & MCB Camp Pendleton \\
\hline 48 & 5L-G422206H & Chevrolet & Express 1500 & 2009 & Van & MCB Camp Pendleton \\
\hline 49 & 5L-G422215H & Chevrolet & Express & 2009 & Van & MCB Camp Pendleton \\
\hline 50 & 5L-G422227H & Ford & F-150 & 2009 & Pickup & MCB Camp Pendleton \\
\hline 51 & $5 \mathrm{~L}-\mathrm{G} 422233 \mathrm{H}$ & Ford & F-150 & 2009 & Pickup & MCB Camp Pendleton \\
\hline 52 & 5L-G422235H & Ford & F-150 & 2009 & Pickup & MCB Camp Pendleton \\
\hline 53 & $5 \mathrm{~L}-\mathrm{G} 422467 \mathrm{~K}$ & Chevrolet & Silverado 1500 & 2010 & Pickup & MCB Camp Pendleton \\
\hline 54 & $5 \mathrm{~L}-\mathrm{G} 422482 \mathrm{~K}$ & Chevrolet & Express & 2010 & Van & MCB Camp Pendleton \\
\hline 55 & 5L-G422486K & Chevrolet & Express & 2010 & Van & MCB Camp Pendleton \\
\hline 56 & 5L-G422785H & Chevrolet & Silverado & 2010 & Pickup & MCB Camp Pendleton \\
\hline 57 & 5L-G422884H & Chevrolet & Silverado & 2010 & Pickup & MCB Camp Pendleton \\
\hline 58 & $5 \mathrm{~L}-\mathrm{G} 422886 \mathrm{H}$ & Chevrolet & Silverado & 2010 & Pickup & MCB Camp Pendleton \\
\hline 59 & $5 \mathrm{M}-\mathrm{G} 106209 \mathrm{H}$ & Pontiac & G6 & 2009 & Sedan & MCAS Miramar \\
\hline 60 & $5 \mathrm{M}-\mathrm{G} 106212 \mathrm{H}$ & Pontiac & G6 & 2009 & Sedan & MCAS Miramar \\
\hline 61 & 5M-G136272L & Ford & Focus & 2012 & Sedan & MCAS Miramar \\
\hline 62 & $5 \mathrm{M}-\mathrm{G} 414171 \mathrm{H}$ & Dodge & Dakota & 2009 & Small pickup & MCAS Miramar \\
\hline 63 & $5 \mathrm{M}-\mathrm{G} 414446 \mathrm{H}$ & Dodge & Dakota & 2009 & Small pickup & MCAS Miramar \\
\hline 64 & $5 \mathrm{M}-\mathrm{G} 414515 \mathrm{~L}$ & Dodge & Grand Caravan & 2011 & Van & MCAS Miramar \\
\hline 65 & $5 \mathrm{M}-\mathrm{G} 414516 \mathrm{~L}$ & Dodge & Grand Caravan & 2011 & Van & MCAS Miramar \\
\hline 66 & $5 \mathrm{M}-\mathrm{G} 414859 \mathrm{~K}$ & Dodge & Grand Caravan & 2010 & Van & MCAS Miramar \\
\hline 67 & $5 \mathrm{M}-\mathrm{G} 414860 \mathrm{~K}$ & Dodge & Grand Caravan & 2010 & Van & MCAS Miramar \\
\hline 68 & $5 \mathrm{M}-\mathrm{G} 422183 \mathrm{G}$ & Chevrolet & Express & 2008 & Van & MCAS Miramar \\
\hline 69 & $5 \mathrm{M}-\mathrm{G} 422205 \mathrm{H}$ & Chevrolet & Express & 2009 & Van & MCAS Miramar \\
\hline 70 & $5 \mathrm{M}-\mathrm{G} 422890 \mathrm{H}$ & Chevrolet & Silverado 1500 & 2010 & Pickup & MCAS Miramar \\
\hline 71 & $5 \mathrm{M}-\mathrm{G} 422891 \mathrm{H}$ & Chevrolet & Silverado 1500 & 2010 & Pickup & MCAS Miramar \\
\hline 72 & $5 \mathrm{M}-\mathrm{G} 423185 \mathrm{H}$ & Chevrolet & Silverado 1500 & 2010 & Pickup & MCAS Miramar \\
\hline
\end{tabular}




\begin{tabular}{ccccccc} 
Vehicle & \multicolumn{5}{c}{ Vehicle Index } \\
No. & Vehicle Label & Make & Model & Year & Type & Base \\
\hline 73 & 5P-G130225A & Honda & Civic & 2009 & Sedan & MCAGCC 29 Palms \\
\hline
\end{tabular}




\section{Appendix B \\ U.S. Navy Vehicle Data Sheets}


Vehicle:

Report Period:

Model Year:

Vehicle Make:

Vehicle Model:

Body Type:

Total Number of Days with Driving

Average Number of Trips

Average Trip Distance

Total Number of Trips

Total Distance (miles)

Total Trip Duration (minutes)
5B-294399

8/1/2014 00:00:00 - 7/1/2015 00:00:00

2010

FORD

FUSION

sedan

\section{Distribution of Daily Vehicle Travel Distance}

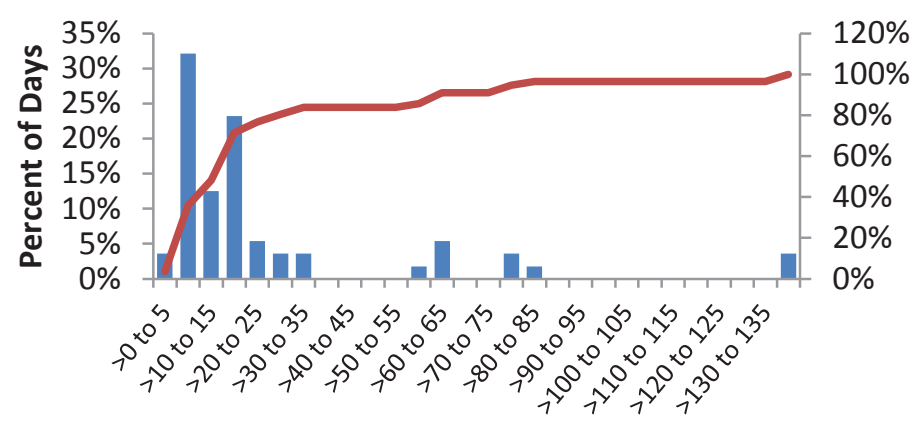

Daily Vehicle Miles Traveled

\section{Daily Vehicle Travel Distance}

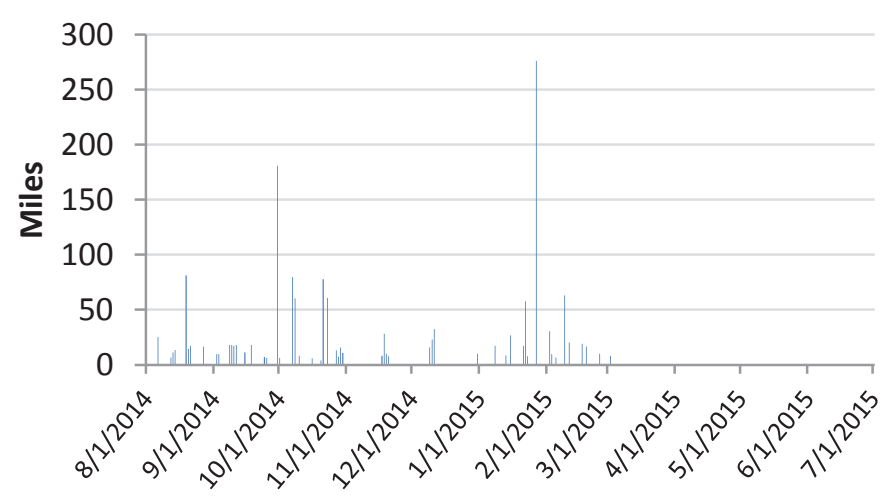

Distribution of Daily Vehicle Travel Times ${ }^{1}$

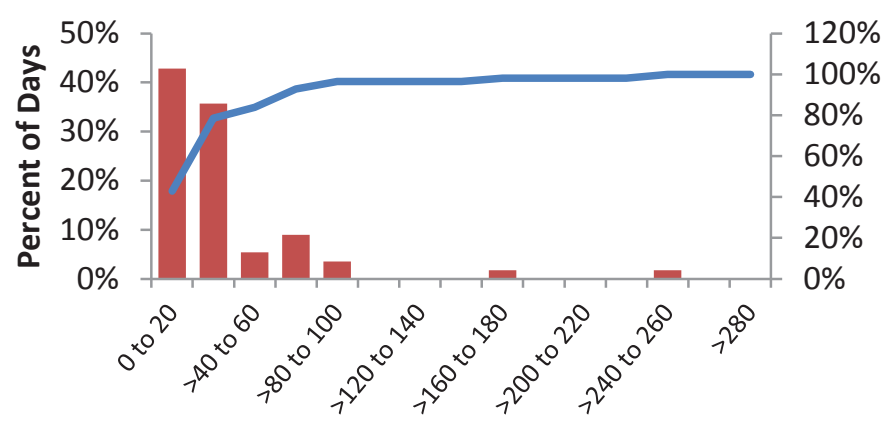

Daily Vehicle Trip Times (min)

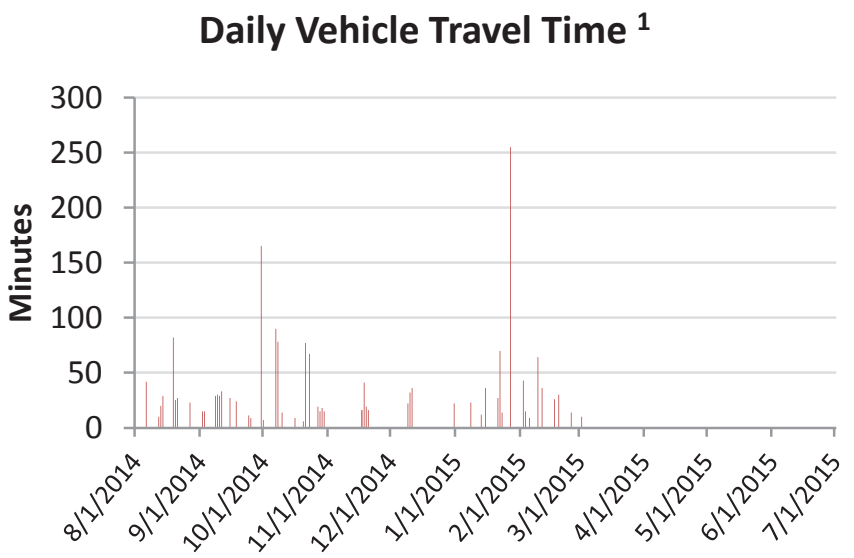

Note 1: Travel time includes all time when vehicle is in the "key on" state, even when the vehicle is not moving

$\begin{array}{cc}\text { Percentage of } & \text { Number of days } \\ \text { days vehicle } & \text { vehicle exceeds }\end{array}$

EV Range

59

63

67 exceeds EV range

$14 \%$

9\%

9\%
EV range

8

5

5 
Vehicle:

Report Period:

Model Year:

Vehicle Make:

Vehicle Model:

Body Type:
5P-G130225A

8/1/2014 00:00:00 - 7/1/2015 00:00:00

2009

Honda

Civic

sedan
Total Number of Days with Driving

Average Number of Trips

Average Trip Distance

Total Number of Trips

Total Distance (miles)

Total Trip Duration (minutes)
274

11.7

18.8

3532

5688.2

23208

\section{Distribution of Daily Vehicle Travel Distance}

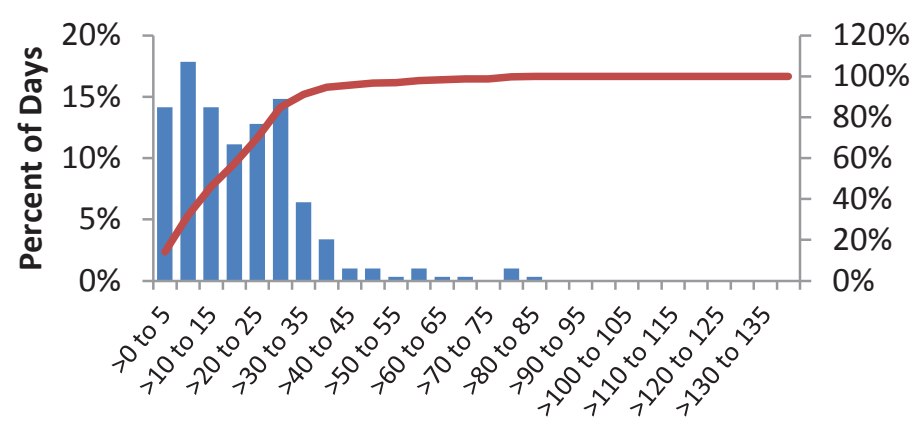

Daily Vehicle Miles Traveled

\section{Daily Vehicle Travel Distance}

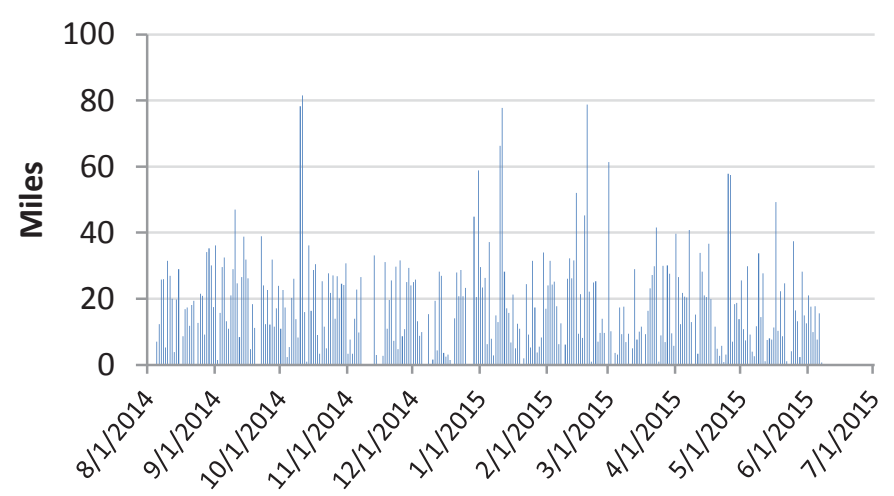

Distribution of Daily Vehicle Travel Times ${ }^{1}$

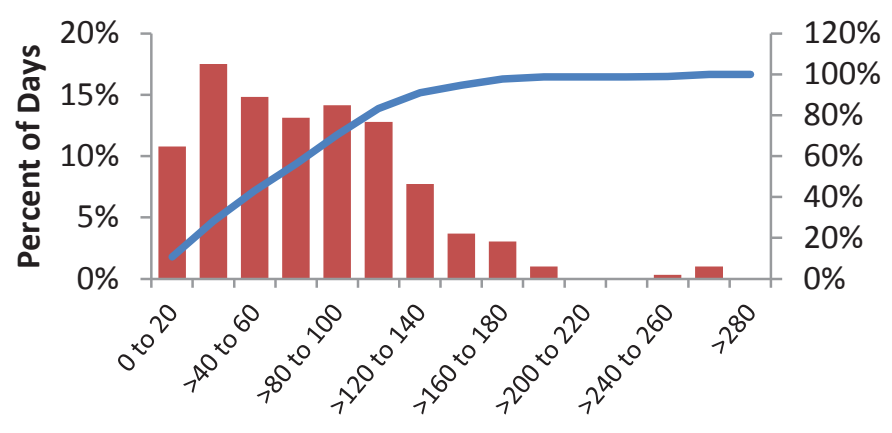

Daily Vehicle Trip Times ( $\min )$

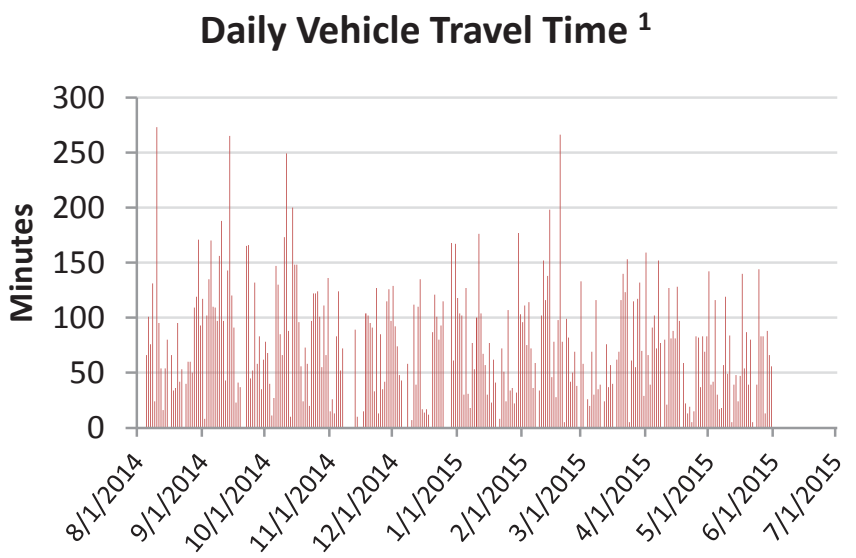

Note 1: Travel time includes all time when vehicle is in the "key on" state, even when the vehicle is not moving

$\begin{array}{cc}\text { Percentage of } & \text { Number of days } \\ \text { days vehicle } & \text { vehicle exceeds }\end{array}$

EV Range

59

63

67 exceeds EV range

$2 \%$

$2 \%$

$1 \%$
EV range

6

5

4 
Vehicle:

Report Period:

Model Year:

Vehicle Make:

Vehicle Model:

Body Type:

Total Number of Days with Driving

Average Number of Trips

Average Trip Distance

Total Number of Trips

Total Distance (miles)

Total Trip Duration (minutes)
5M-G423185H

8/1/2014 00:00:00 - 7/1/2015 00:00:00

2010

Chevrolet

Silverado 1500

pickup

\section{Distribution of Daily Vehicle Travel Distance}

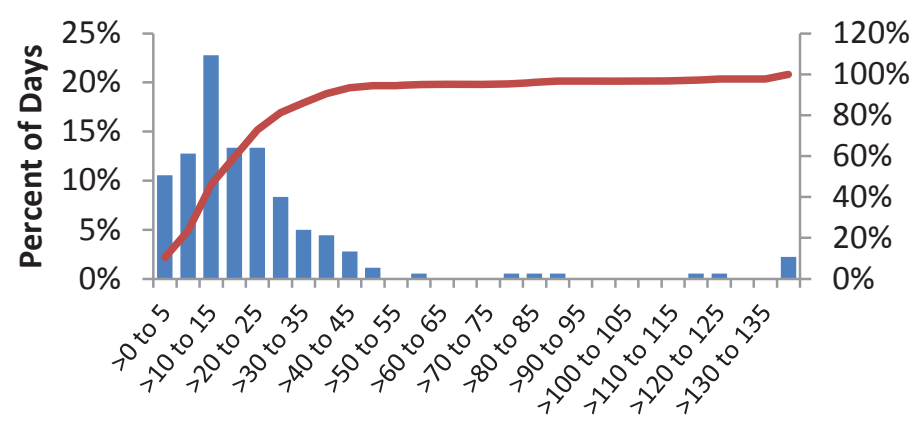

Daily Vehicle Miles Traveled

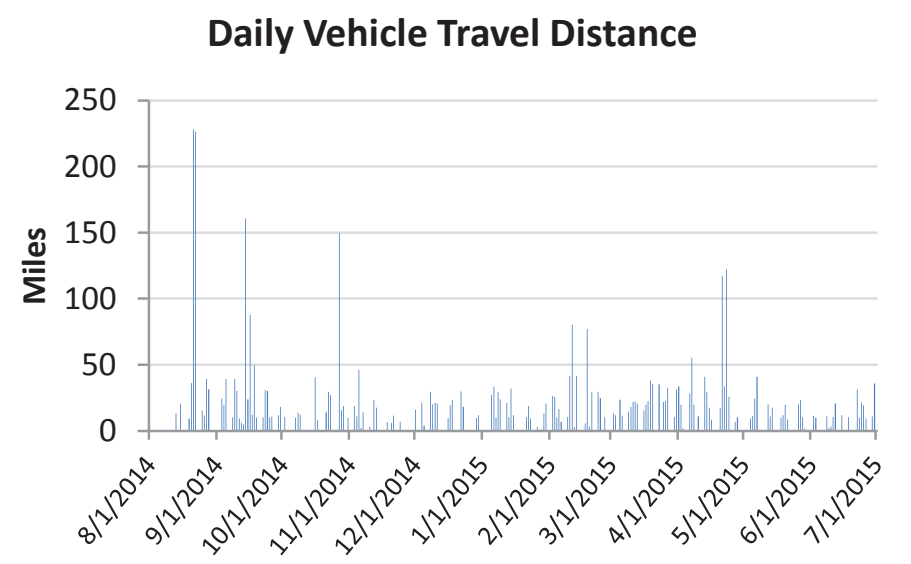

180

5.8

23.8

1042

4289.9

11324

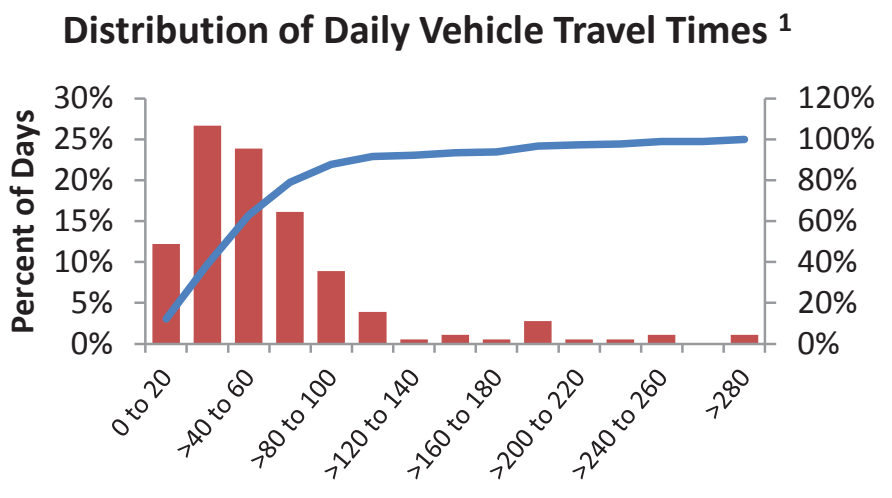

Daily Vehicle Trip Times (min)

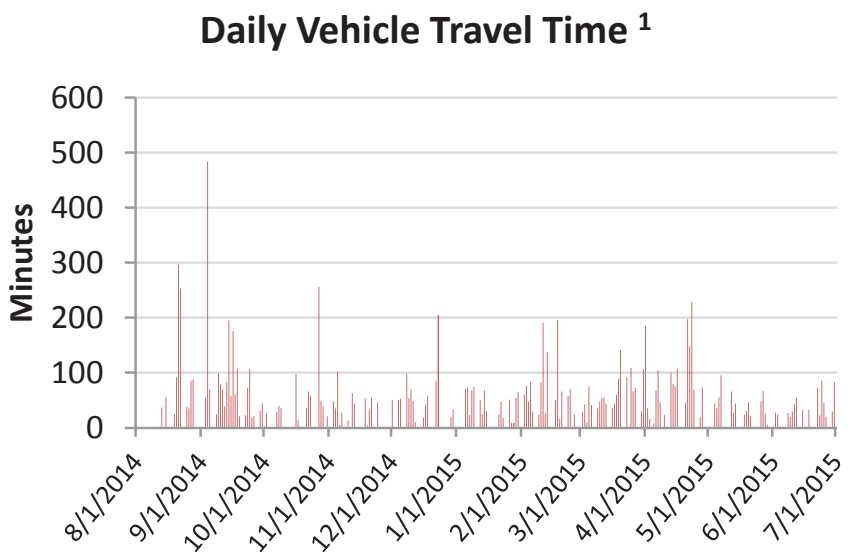

Note 1: Travel time includes all time when vehicle is in the "key on" state, even when the vehicle is not moving

$\begin{array}{ccc}\text { EV Range } & \begin{array}{c}\text { Percentage of } \\ \text { days vehicle } \\ \text { exceeds EV range }\end{array} & \begin{array}{c}\text { Number of days } \\ \text { vehicle exceeds } \\ \text { EV range }\end{array} \\ 69 & 5 \% & 9 \\ 63 & 5 \% & 9 \\ 67 & 5 \% & 9\end{array}$


Vehicle:

Report Period:

Model Year:

Vehicle Make:

Vehicle Model:

Body Type:

Total Number of Days with Driving

Average Number of Trips

Average Trip Distance

Total Number of Trips

Total Distance (miles)

Total Trip Duration (minutes)
5M-G422891H

8/1/2014 00:00:00 - 7/1/2015 00:00:00

2010

Chevrolet

Silverado 1500

pickup

\section{Distribution of Daily Vehicle Travel Distance}

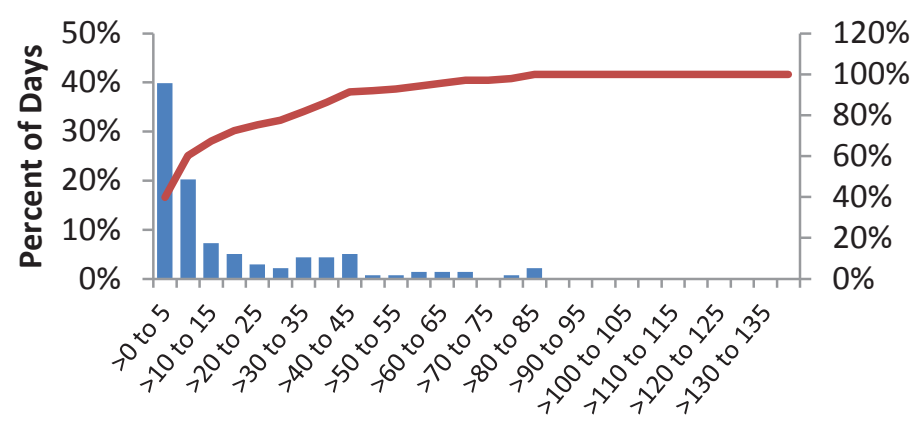

Daily Vehicle Miles Traveled

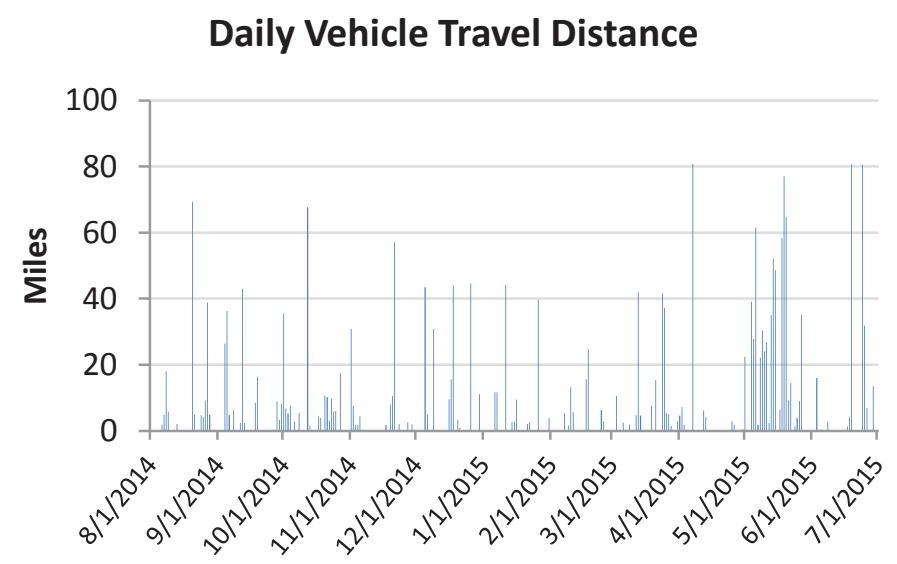

138

6.8

16.6

944

2286.4

10098

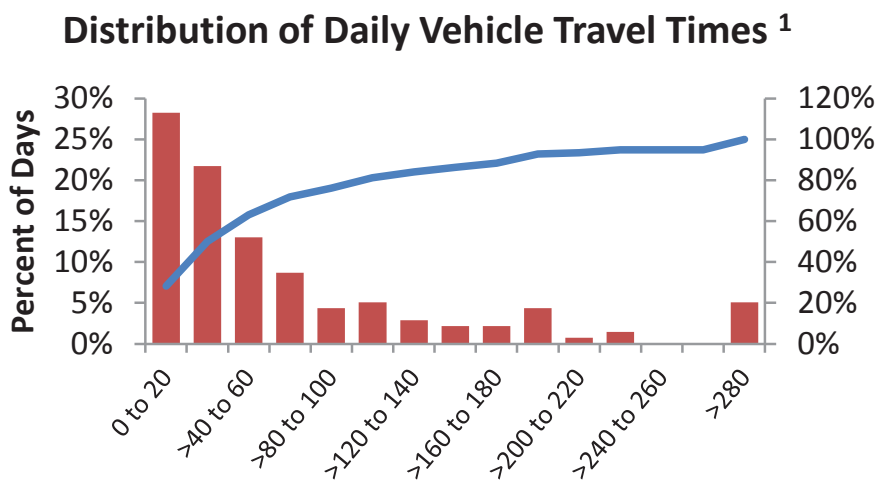

Daily Vehicle Trip Times (min)

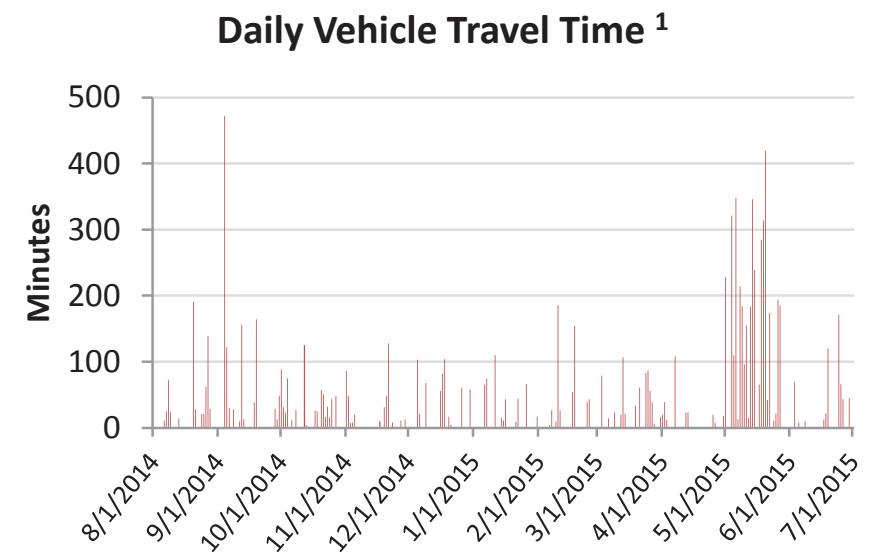

Note 1: Travel time includes all time when vehicle is in the "key on" state, even when the vehicle is not moving

$\begin{array}{ccc}\text { EV Range } & \begin{array}{c}\text { Percentage of } \\ \text { days vehicle } \\ \text { exceeds EV range }\end{array} & \begin{array}{c}\text { Number of days } \\ \text { vehicle exceeds } \\ \text { EV range }\end{array} \\ 69 & 6 \% & 8 \\ 63 & 5 \% & 7 \\ 67 & 4 \% & 6\end{array}$


Vehicle:

Report Period:

Model Year:

Vehicle Make:

Vehicle Model:

Body Type:

Total Number of Days with Driving

Average Number of Trips

Average Trip Distance

Total Number of Trips

Total Distance (miles)

Total Trip Duration (minutes)
5M-G422890H

8/1/2014 00:00:00 - 7/1/2015 00:00:00

2010

Chevrolet

Silverado 1500

pickup

\section{Distribution of Daily Vehicle Travel Distance}

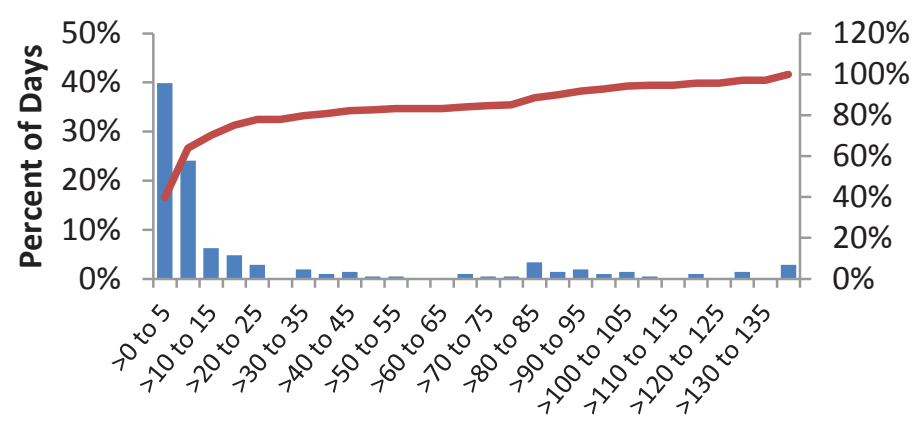

Daily Vehicle Miles Traveled

\section{Daily Vehicle Travel Distance}

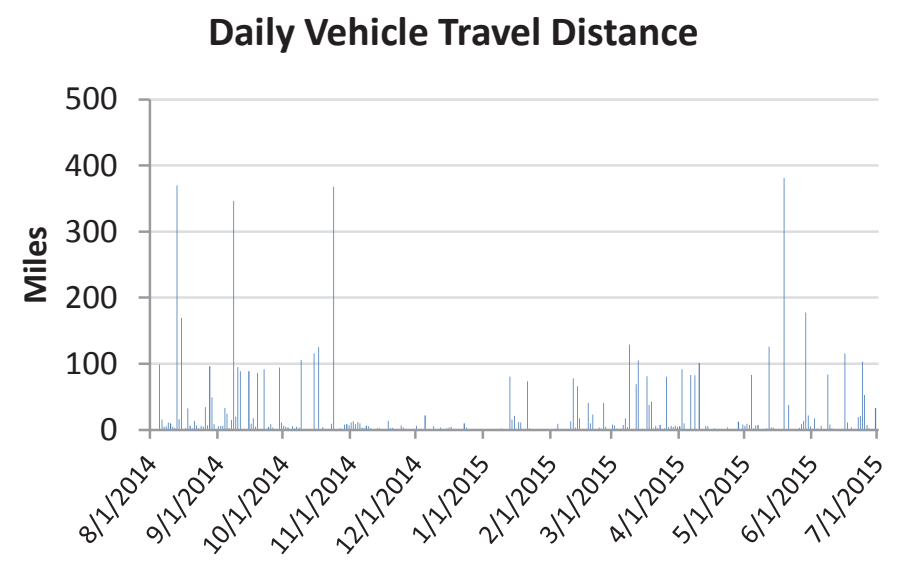

208

5.8

28.8

1201

5984.4

17740
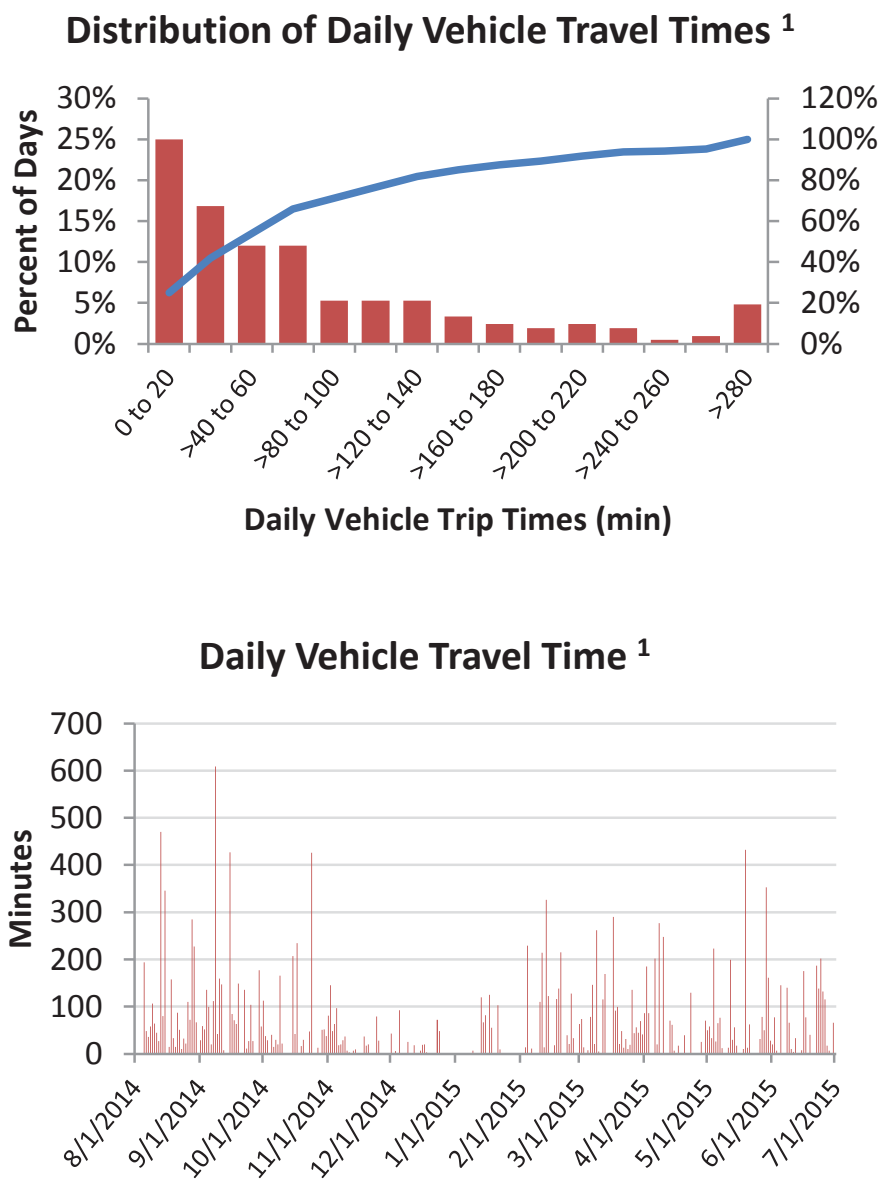

Note 1: Travel time includes all time when vehicle is in the "key on" state, even when the vehicle is not moving

$\begin{array}{ccc}\text { EV Range } & \begin{array}{c}\text { Percentage of } \\ \text { days vehicle } \\ \text { exceeds EV range }\end{array} & \begin{array}{c}\text { Number of days } \\ \text { vehicle exceeds } \\ \text { EV range }\end{array} \\ 59 & 17 \% & 35 \\ 63 & 17 \% & 35 \\ 67 & 16 \% & 34\end{array}$


Vehicle:

Report Period:

Model Year:

Vehicle Make:

Vehicle Model:

Body Type:
5M-G422205H

8/1/2014 00:00:00 - 7/1/2015 00:00:00

2009

Chevrolet

Express

van
Total Number of Days with Driving

Average Number of Trips

Average Trip Distance

Total Number of Trips

Total Distance (miles)

Total Trip Duration (minutes)
170

4.7

46.9

807

7975.0

14473

\section{Distribution of Daily Vehicle Travel Distance}

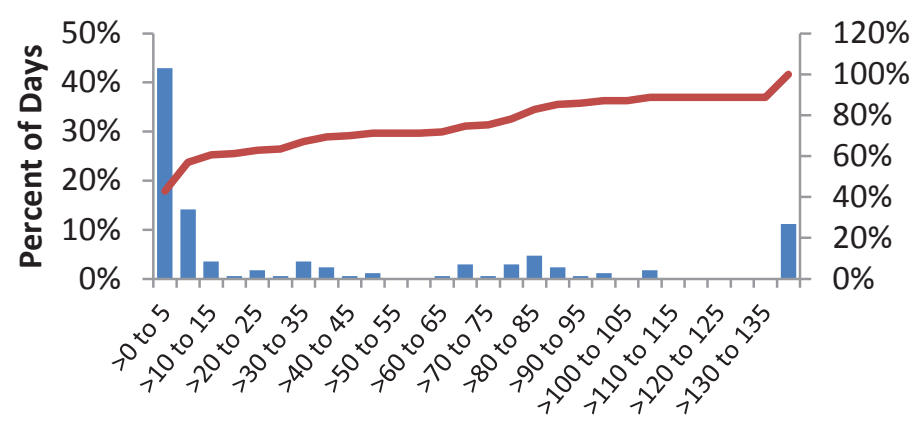

Daily Vehicle Miles Traveled

\section{Daily Vehicle Travel Distance}

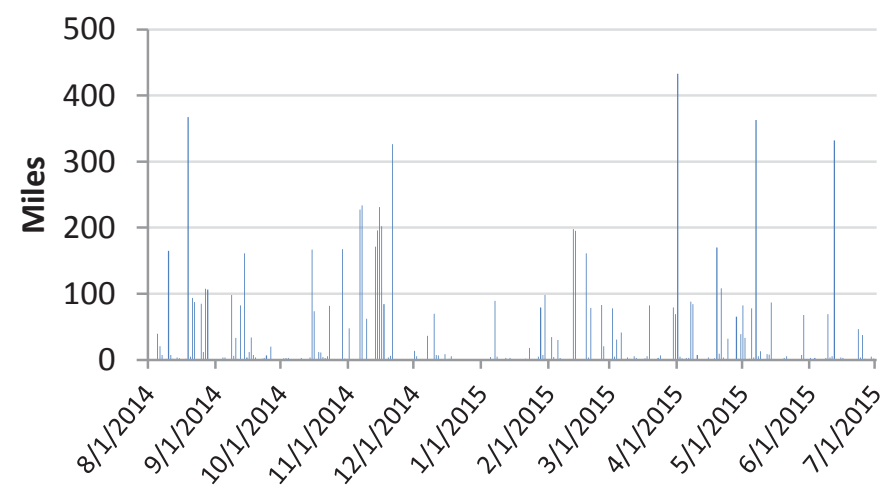

Distribution of Daily Vehicle Travel Times ${ }^{1}$

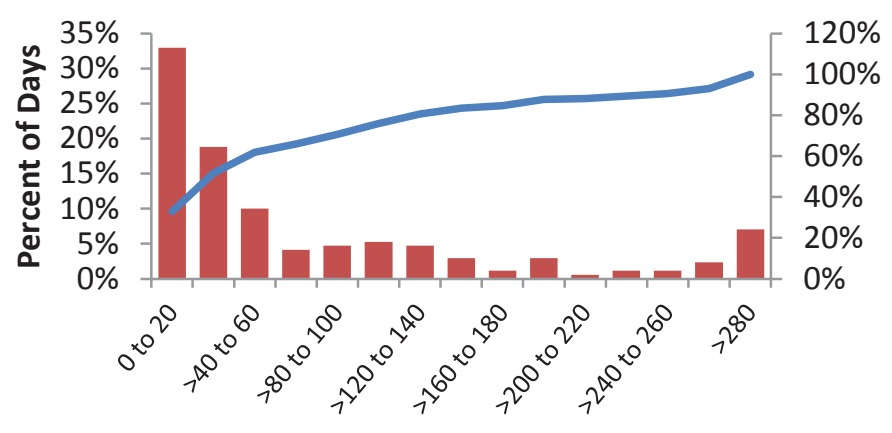

Daily Vehicle Trip Times (min)

\section{Daily Vehicle Travel Time ${ }^{1}$}

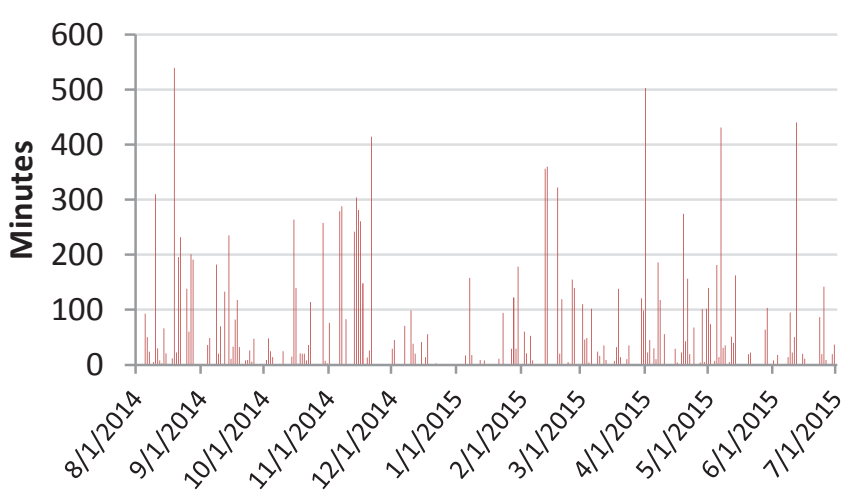

Note 1: Travel time includes all time when vehicle is in the "key on" state, even when the vehicle is not moving

$\begin{array}{cc}\text { Percentage of } & \text { Number of days } \\ \text { days vehicle } & \text { vehicle exceeds }\end{array}$

EV Range

59

63

67 exceeds EV range

$29 \%$

$28 \%$

$28 \%$
EV range

49

48

47 
Vehicle:

Report Period:

Model Year:

Vehicle Make:

Vehicle Model:

Body Type:
5M-G422183G

8/1/2014 00:00:00 - 7/1/2015 00:00:00

2008

Chevrolet

Express

van

Total Number of Days with Driving

Average Number of Trips

4.2

Average Trip Distance

50.6

Total Number of Trips

381

Total Distance (miles)

4605.6

Total Trip Duration (minutes)

8894

\section{Distribution of Daily Vehicle Travel Distance}

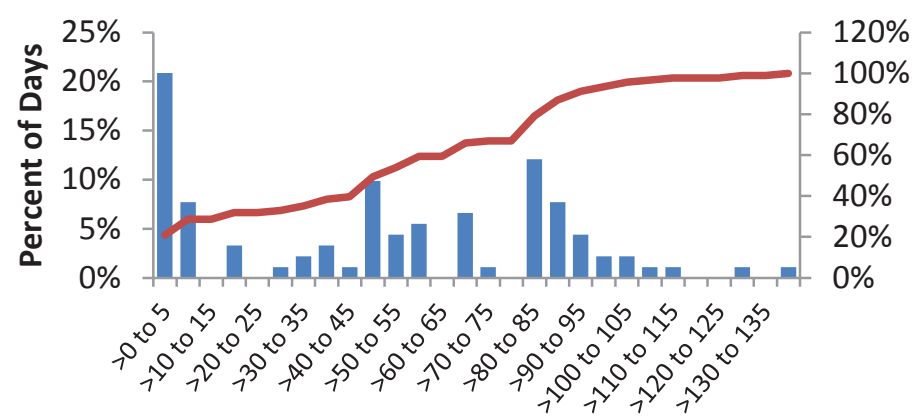

Daily Vehicle Miles Traveled

\section{Daily Vehicle Travel Distance}

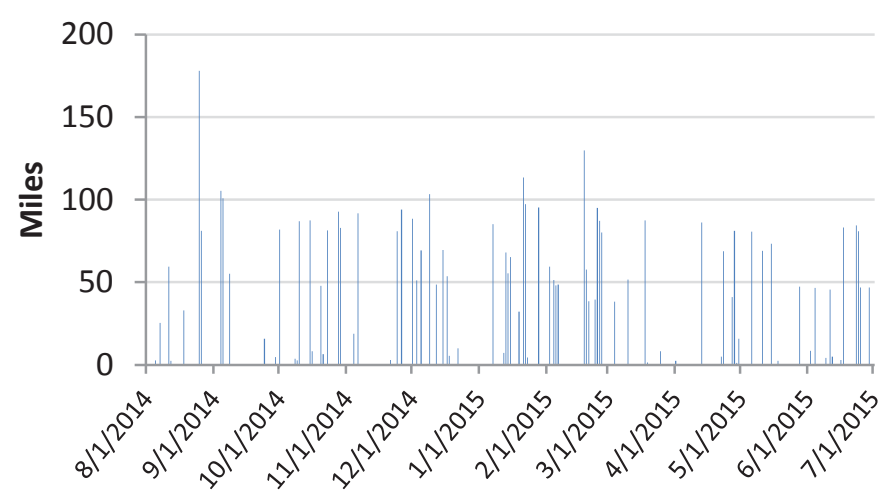

Distribution of Daily Vehicle Travel Times ${ }^{1}$

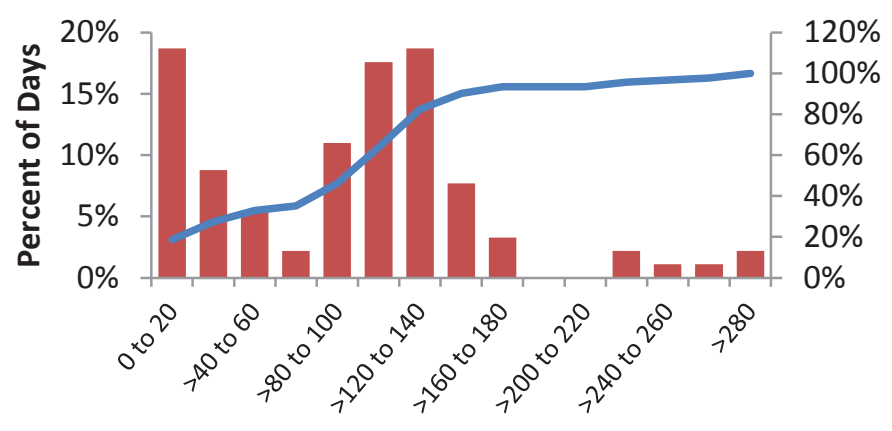

Daily Vehicle Trip Times (min)

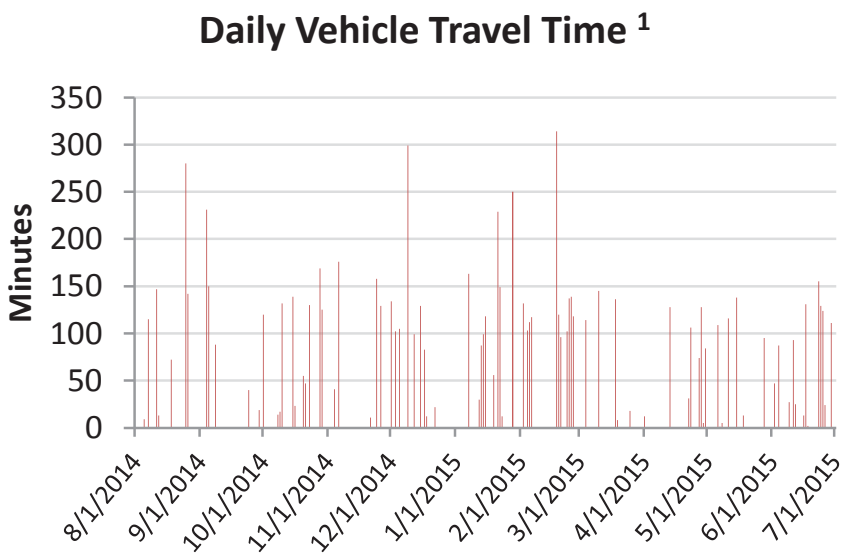

Note 1: Travel time includes all time when vehicle is in the "key on" state, even when the vehicle is not moving

$\begin{array}{cc}\text { Percentage of } & \text { Number of days } \\ \text { days vehicle } & \text { vehicle exceeds }\end{array}$

EV Range

59

63

67 exceeds EV range

$43 \%$

$41 \%$

$40 \%$
EV range

39

37

36 
Vehicle:

Report Period:

Model Year:

Vehicle Make:

Vehicle Model:

Body Type:
5M-G414860K

8/1/2014 00:00:00 - 7/1/2015 00:00:00

2010

Dodge

Grand Caravan

van
Total Number of Days with Driving

Average Number of Trips

Average Trip Distance

Total Number of Trips

Total Distance (miles)

Total Trip Duration (minutes)
147

4.6

21.5

680

3159.7

7828

\section{Distribution of Daily Vehicle Travel Distance}

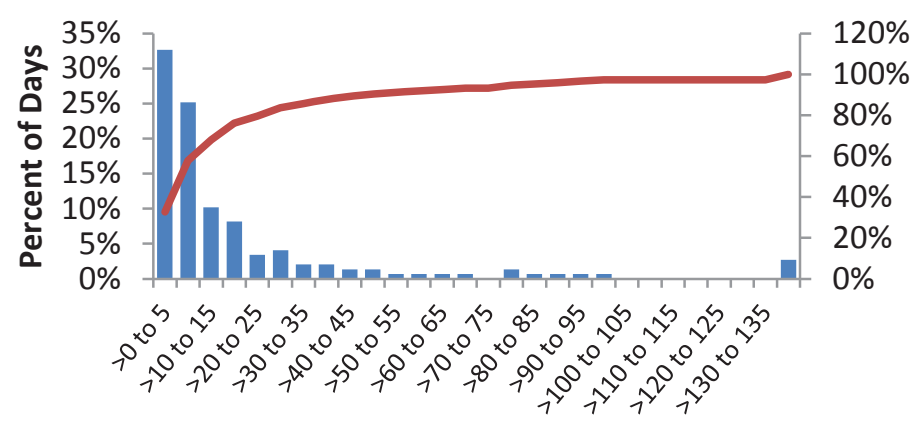

Daily Vehicle Miles Traveled

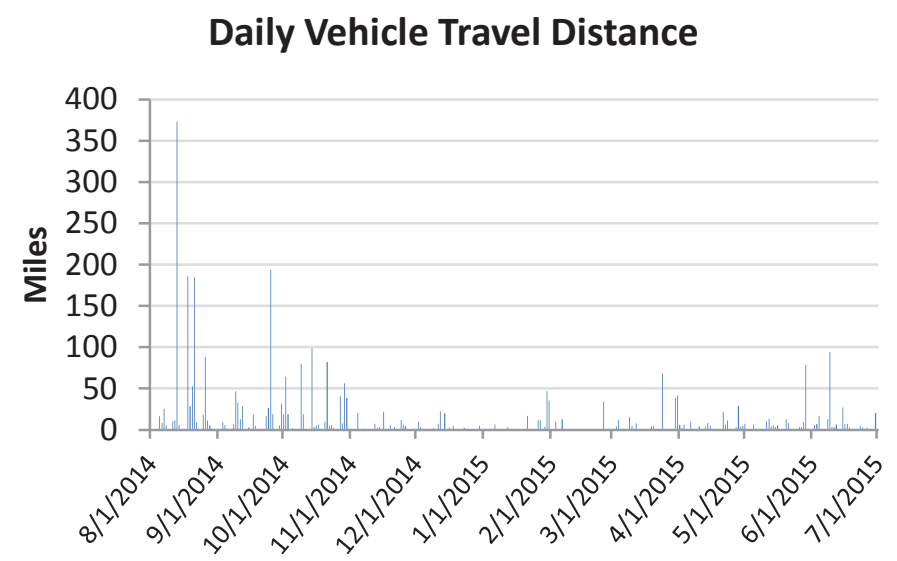

\section{Distribution of Daily Vehicle Travel Times ${ }^{1}$}

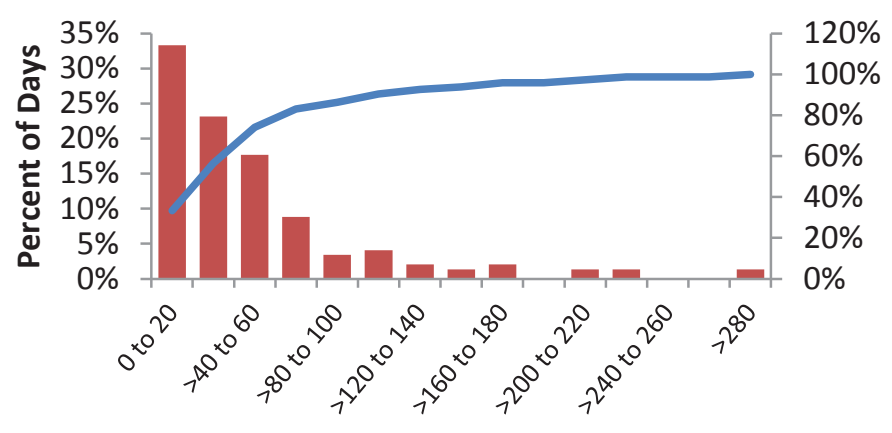

Daily Vehicle Trip Times (min)

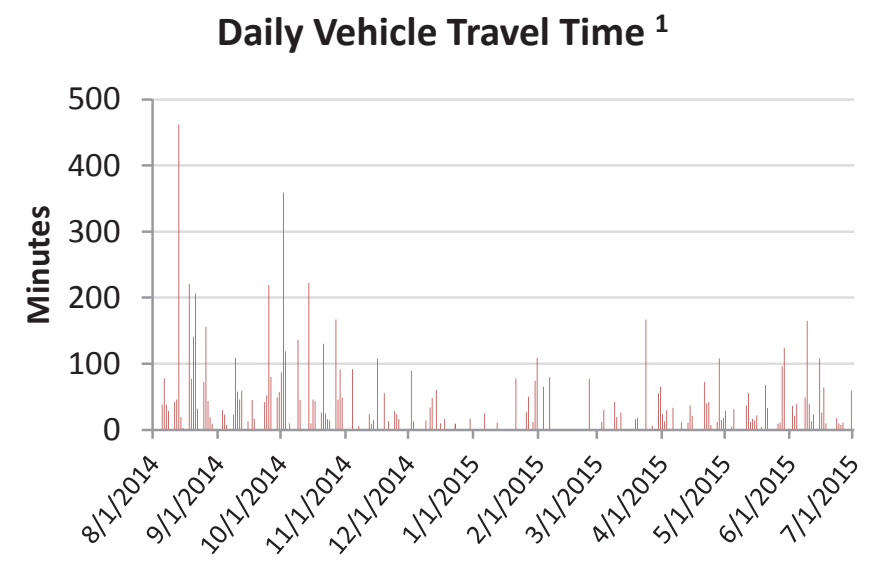

Note 1: Travel time includes all time when vehicle is in the "key on" state, even when the vehicle is not moving

$\begin{array}{cc}\text { Percentage of } & \text { Number of days } \\ \text { days vehicle } & \text { vehicle exceeds }\end{array}$

EV Range

59

63

67 exceeds EV range

$8 \%$

$8 \%$

$7 \%$
EV range

12

12

11 
Vehicle:

Report Period:

Model Year:

Vehicle Make:

Vehicle Model:

Body Type:
5M-G414859K

8/1/2014 00:00:00 - 7/1/2015 00:00:00

2010

Dodge

Grand Caravan

van
Total Number of Days with Driving Average Number of Trips

Average Trip Distance

Total Number of Trips

Total Distance (miles)

Total Trip Duration (minutes)
85

5.8

87.2

495

7412.2

13637

\section{Distribution of Daily Vehicle Travel Distance}

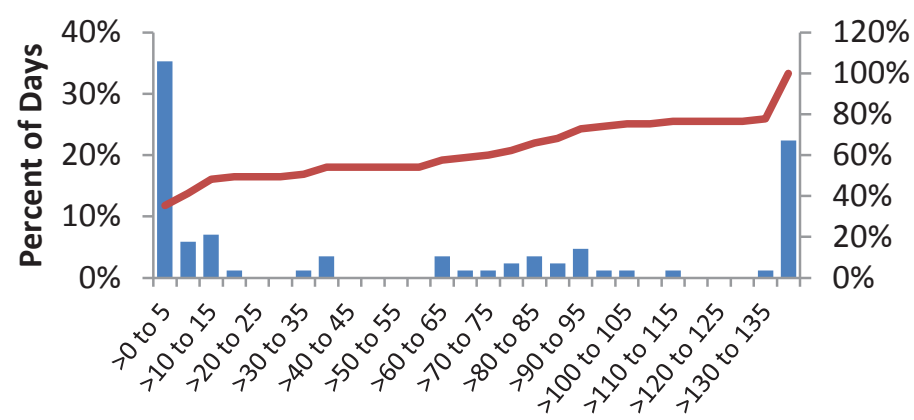

Daily Vehicle Miles Traveled

\section{Daily Vehicle Travel Distance}

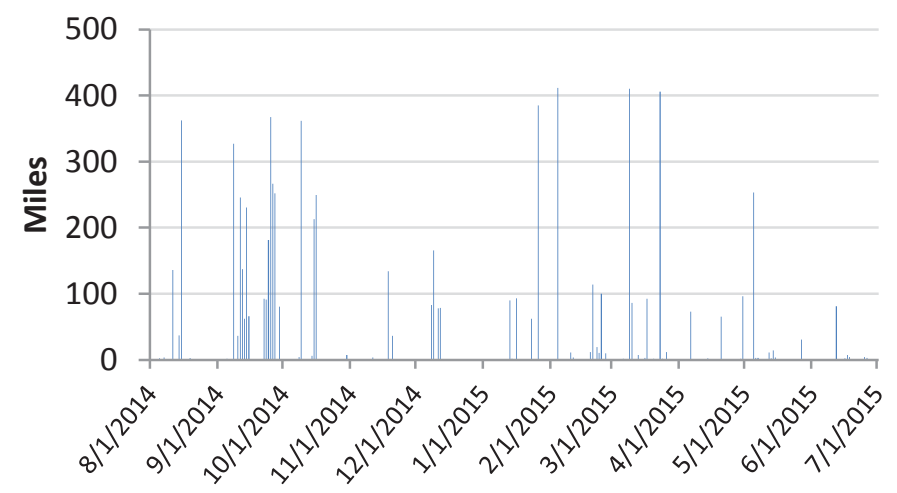

\section{Distribution of Daily Vehicle Travel Times ${ }^{1}$}

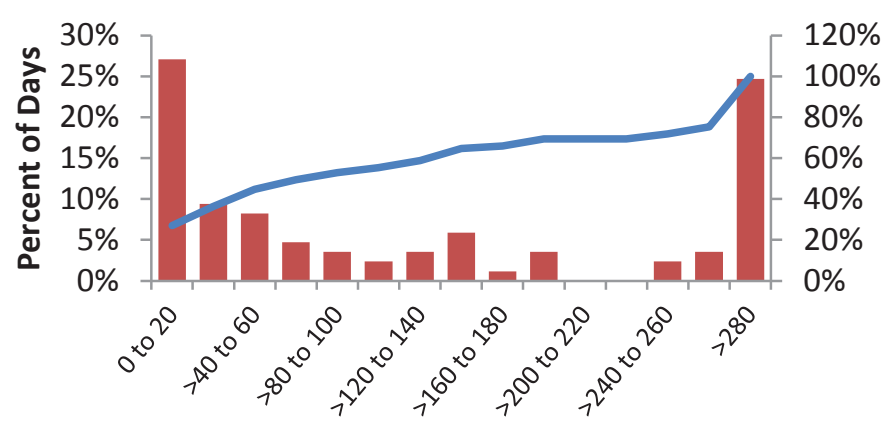

Daily Vehicle Trip Times (min)

\section{Daily Vehicle Travel Time ${ }^{1}$}

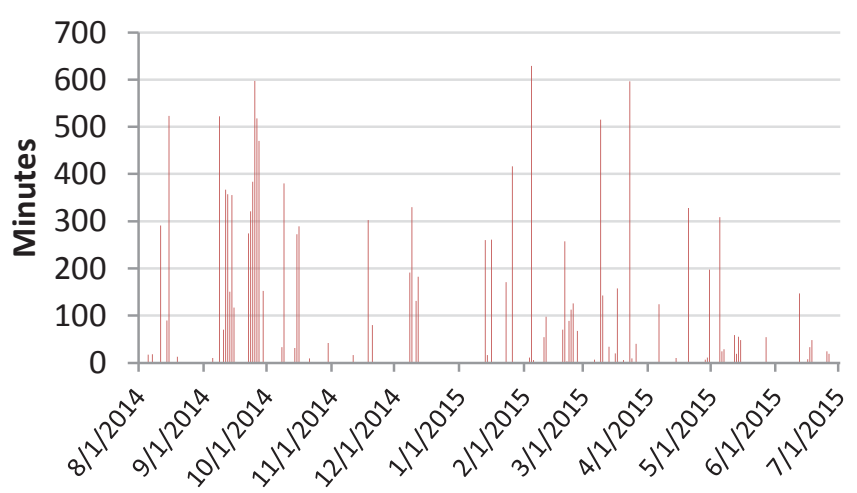

Note 1: Travel time includes all time when vehicle is in the "key on" state, even when the vehicle is not moving

Percentage of Number of days
days vehicle

EV Range

59

63

67 exceeds EV range

$46 \%$

$44 \%$

$41 \%$
EV range

39

37

35 
Vehicle:

Report Period:

Model Year:

Vehicle Make:

Vehicle Model:

Body Type:
5M-G414516L

8/1/2014 00:00:00 - 7/1/2015 00:00:00

2011

Dodge

Grand Caravan

van

Total Number of Days with Driving

Average Number of Trips

Average Trip Distance

Total Number of Trips

565

Total Distance (miles)

1764.4

Total Trip Duration (minutes)

\section{Distribution of Daily Vehicle Travel Distance}

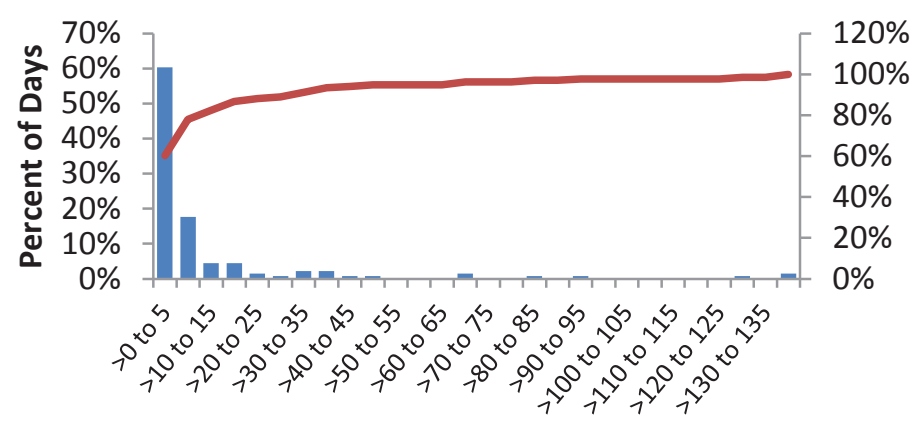

Daily Vehicle Miles Traveled

\section{Daily Vehicle Travel Distance}

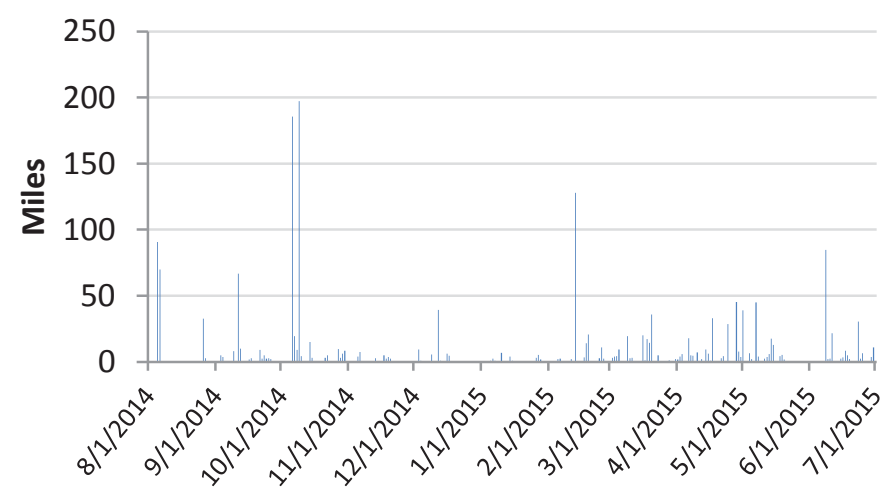

Distribution of Daily Vehicle Travel Times ${ }^{1}$

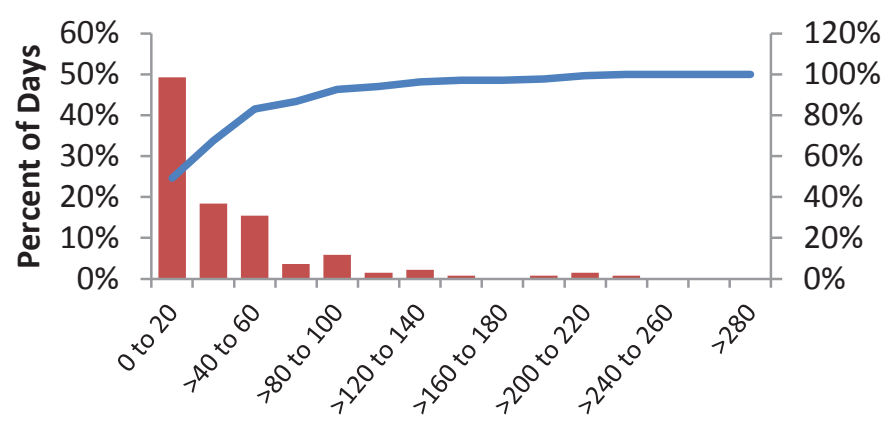

Daily Vehicle Trip Times (min)

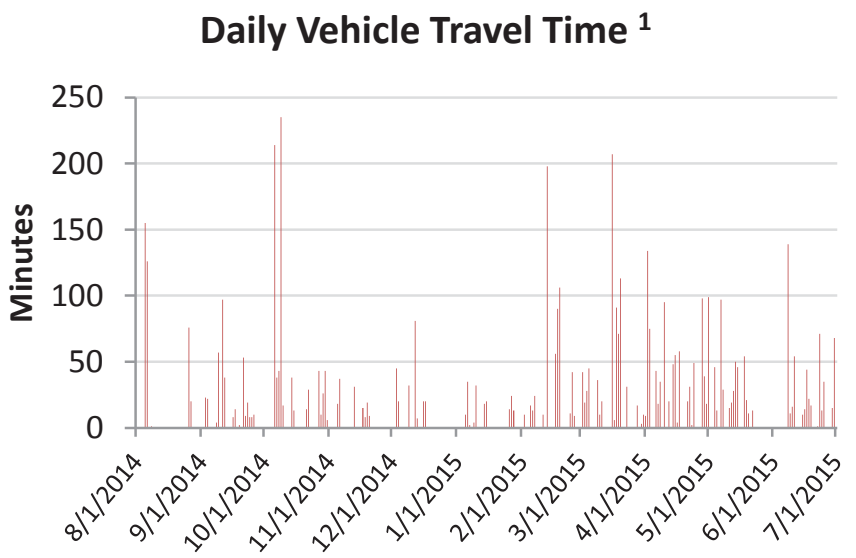

Note 1: Travel time includes all time when vehicle is in the "key on" state, even when the vehicle is not moving

$\begin{array}{cc}\text { Percentage of } & \text { Number of days } \\ \text { days vehicle } & \text { vehicle exceeds }\end{array}$

EV Range

59

63

67 exceeds EV range

$5 \%$

$5 \%$

$4 \%$
EV range

7

7

6 
Vehicle:

Report Period:

Model Year:

Vehicle Make:

Vehicle Model:

Body Type:
5M-G414515L

8/1/2014 00:00:00 - 7/1/2015 00:00:00

2011

Dodge

Grand Caravan

van

Total Number of Days with Driving

Average Number of Trips

0.0

Average Trip Distance

0.0

Total Number of Trips

Total Distance (miles)

0.0

Total Trip Duration (minutes)

\section{Distribution of Daily Vehicle Travel Times ${ }^{1}$}

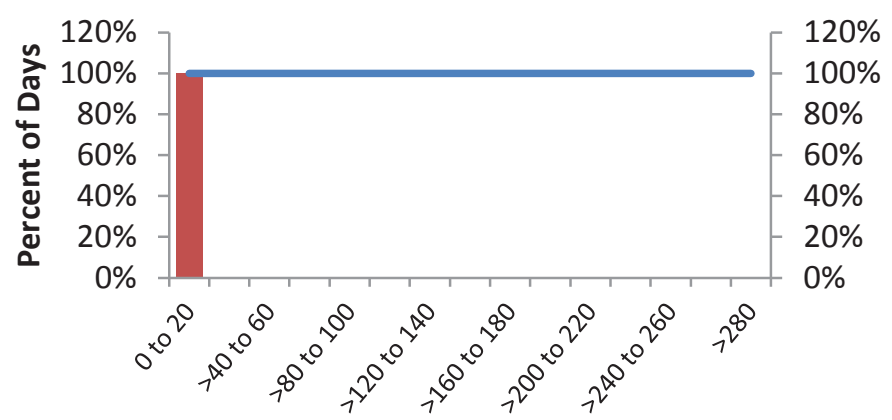

Daily Vehicle Trip Times (min)

Daily Vehicle Miles Traveled

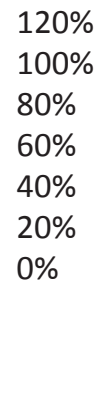

$100 \%$

$80 \%$

$60 \%$

$40 \%$

$20 \%$

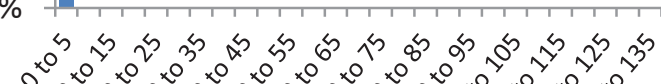

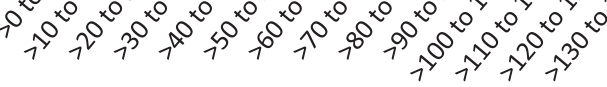

\section{Daily Vehicle Travel Distance}

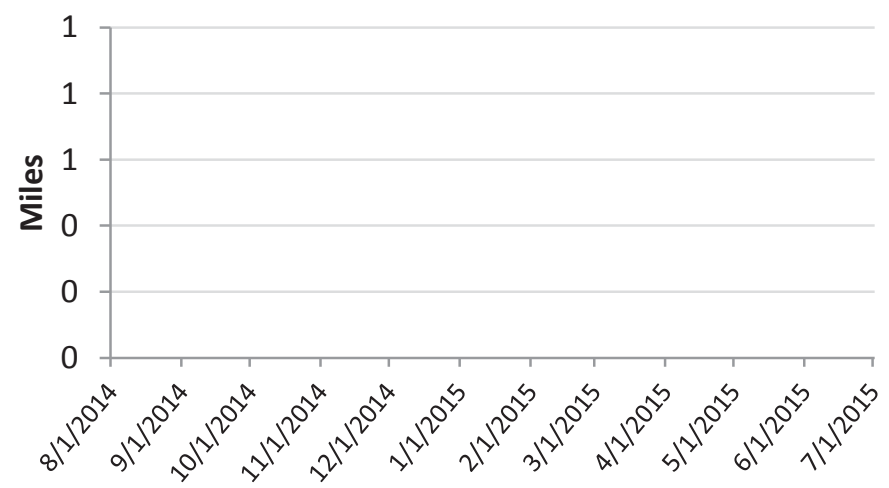

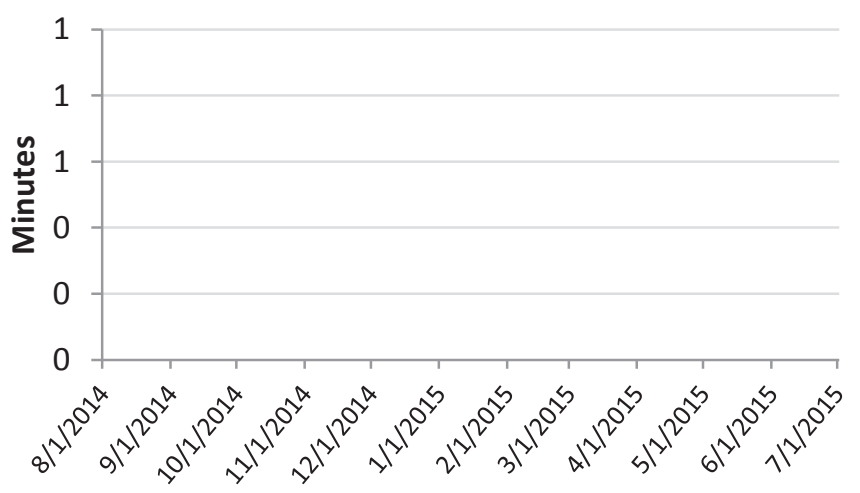

\section{Daily Vehicle Travel Time ${ }^{1}$}

Note 1: Travel time includes all time when vehicle is in the "key on" state, even when the vehicle is not moving

Percentage of Number of days
days vehicle

EV Range

59

63

67 exceeds EV range

$0 \%$

$0 \%$

$0 \%$
EV range

0

0

0 
Vehicle:

Report Period:

Model Year:

Vehicle Make:

Vehicle Model:

Body Type:

Total Number of Days with Driving

Average Number of Trips

Average Trip Distance

Total Number of Trips

Total Distance (miles)

Total Trip Duration (minutes)
5M-G414446H

8/1/2014 00:00:00 - 7/1/2015 00:00:00

2009

Dodge

Dakota

small pickup

\section{Distribution of Daily Vehicle Travel Distance}

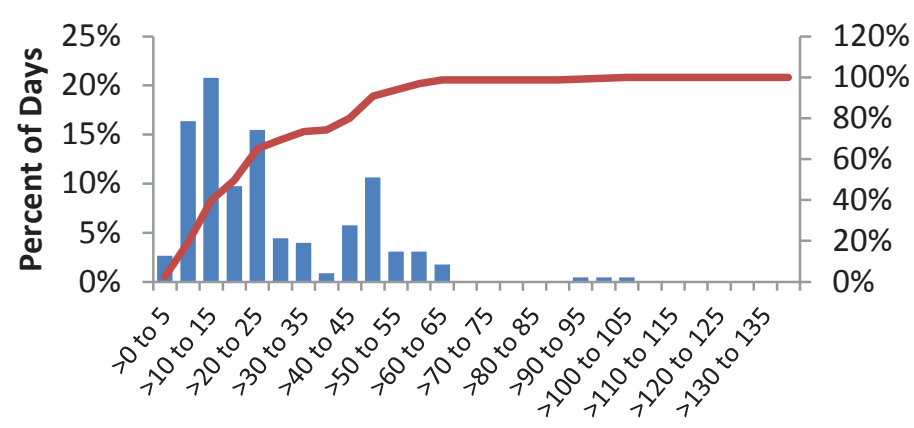

Daily Vehicle Miles Traveled

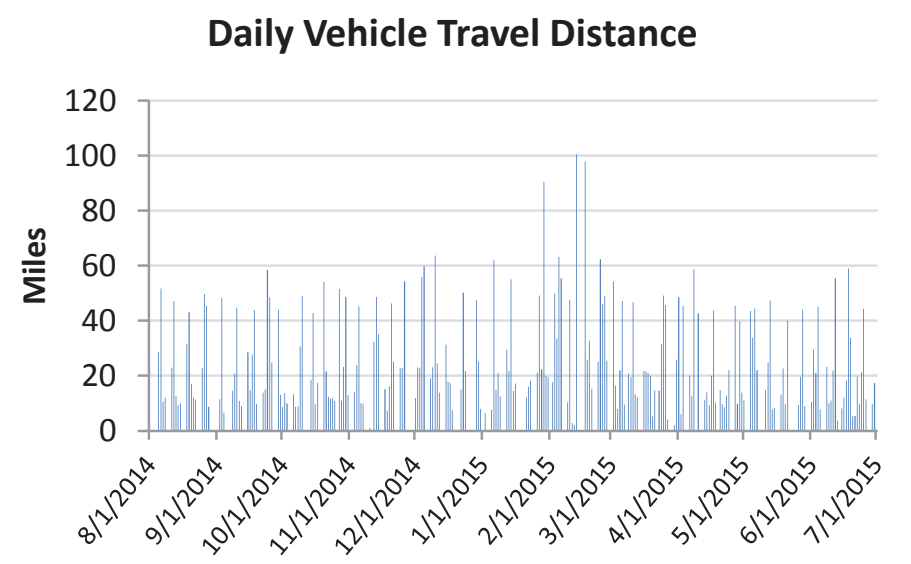

226

12.7

25.4

2859

5743.2

25967
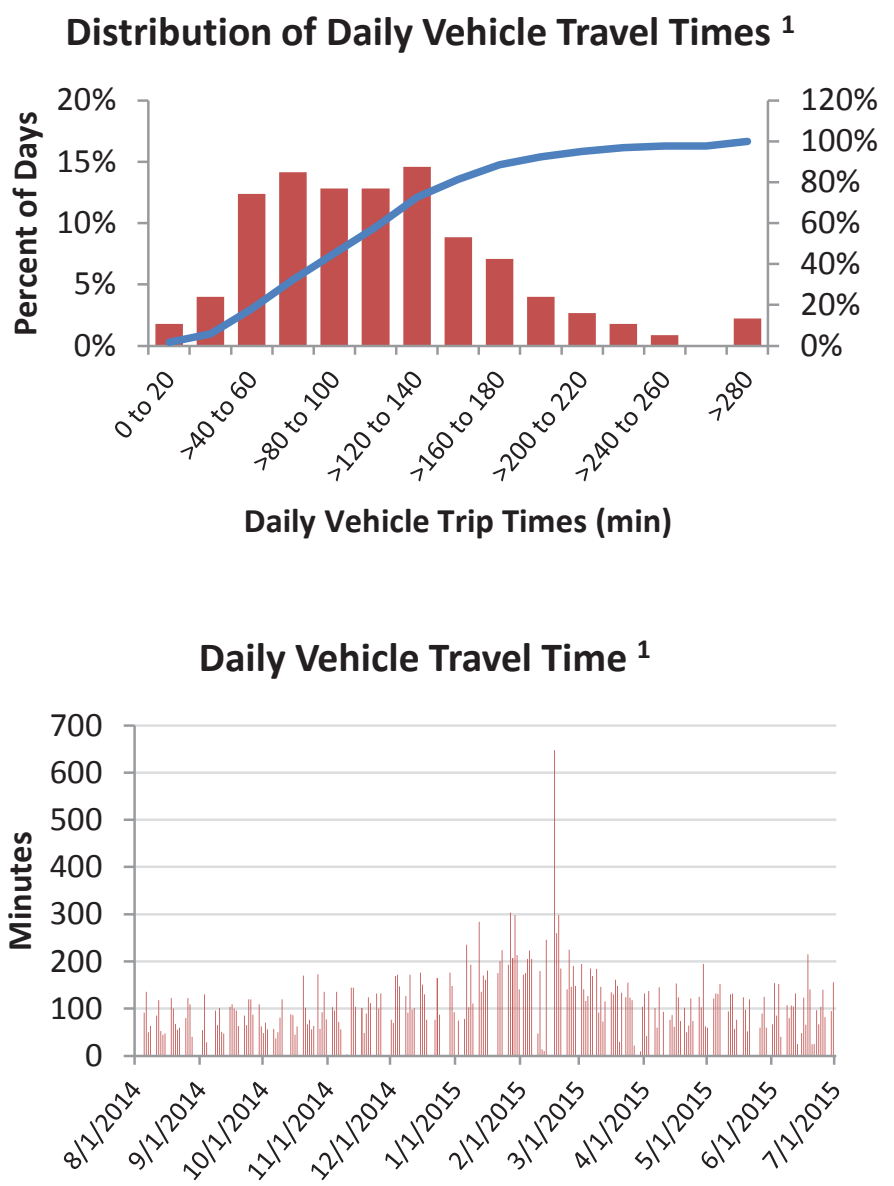

Note 1: Travel time includes all time when vehicle is in the "key on" state, even when the vehicle is not moving

$\begin{array}{ccc}\text { EV Range } & \begin{array}{c}\text { Percentage of } \\ \text { days vehicle } \\ \text { exceeds EV range }\end{array} & \begin{array}{c}\text { Number of days } \\ \text { vehicle exceeds } \\ \text { EV range }\end{array} \\ 59 & 4 \% & 8 \\ 63 & 2 \% & 5 \\ 67 & 1 \% & 3\end{array}$


Vehicle:

Report Period:

Model Year:

Vehicle Make:

Vehicle Model:

Body Type:

Total Number of Days with Driving

Average Number of Trips

Average Trip Distance

Total Number of Trips

Total Distance (miles)

Total Trip Duration (minutes)
5M-G414171H

8/1/2014 00:00:00 - 7/1/2015 00:00:00

2009

Dodge

Dakota

small pickup

\section{Distribution of Daily Vehicle Travel Distance}

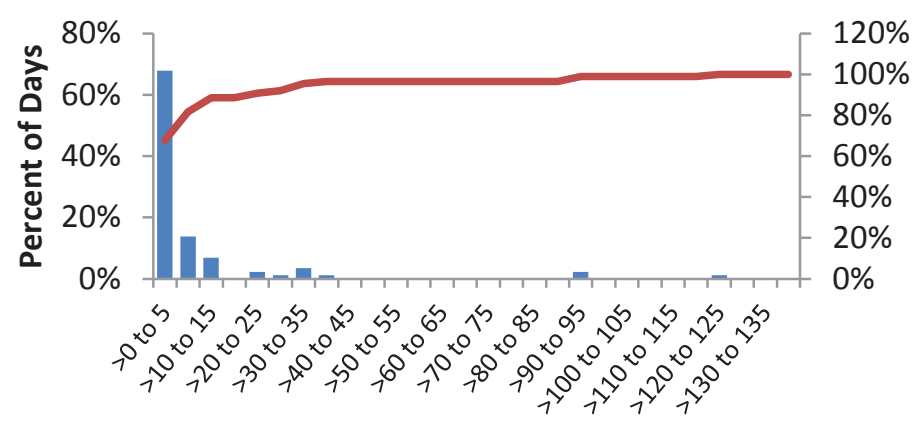

Daily Vehicle Miles Traveled

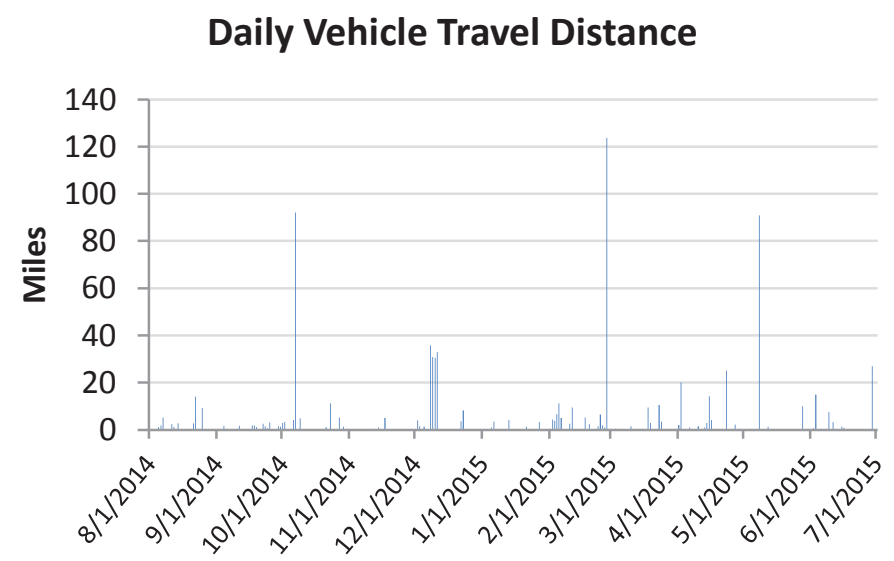

87

3.4

9.2

298

799.5

2064

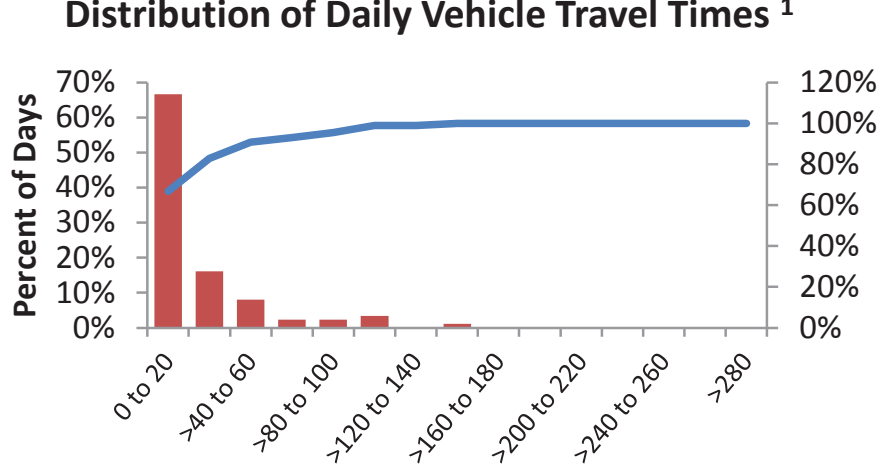

Daily Vehicle Trip Times (min)

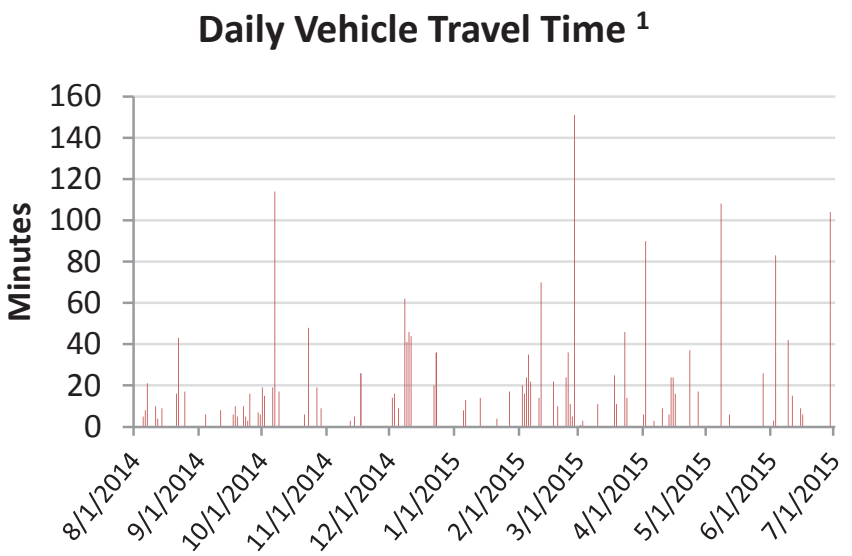

Note 1: Travel time includes all time when vehicle is in the "key on" state, even when the vehicle is not moving

$$
\begin{array}{cc}
\begin{array}{c}
\text { Percentage of } \\
\text { days vehicle }
\end{array} & \text { vehicle exceeds }
\end{array}
$$

EV Range

59

63

67 exceeds EV range

$3 \%$

$3 \%$

$3 \%$
EV range

\section{3}

3

3 
Vehicle:

Report Period:

Model Year:

Vehicle Make:

Vehicle Model:

Body Type:

Total Number of Days with Driving

Average Number of Trips

Average Trip Distance

Total Number of Trips

Total Distance (miles)

Total Trip Duration (minutes)
5M-G136272L

8/1/2014 00:00:00 - 7/1/2015 00:00:00

2012

FORD

Focus

sedan

\section{Distribution of Daily Vehicle Travel Distance}

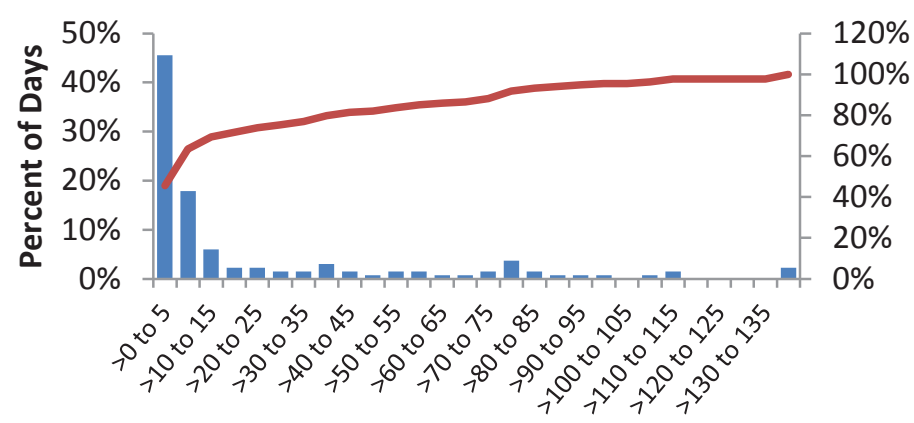

Daily Vehicle Miles Traveled

\section{Daily Vehicle Travel Distance}

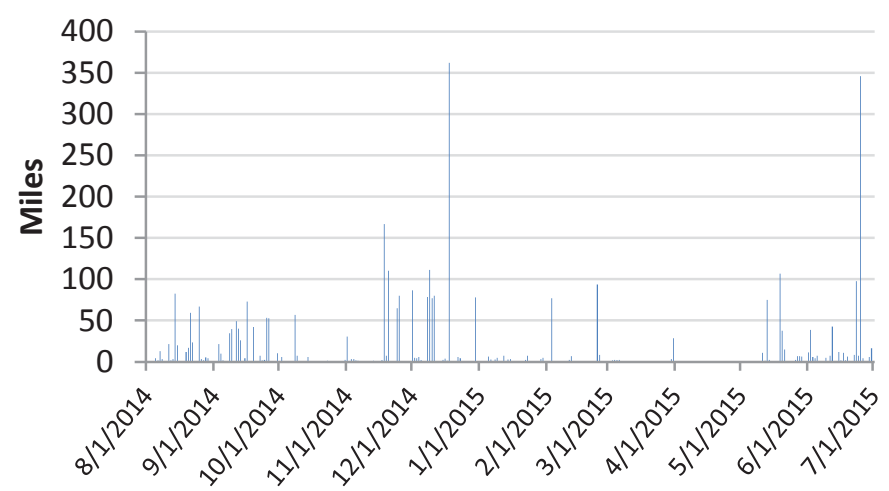

Distribution of Daily Vehicle Travel Times ${ }^{1}$

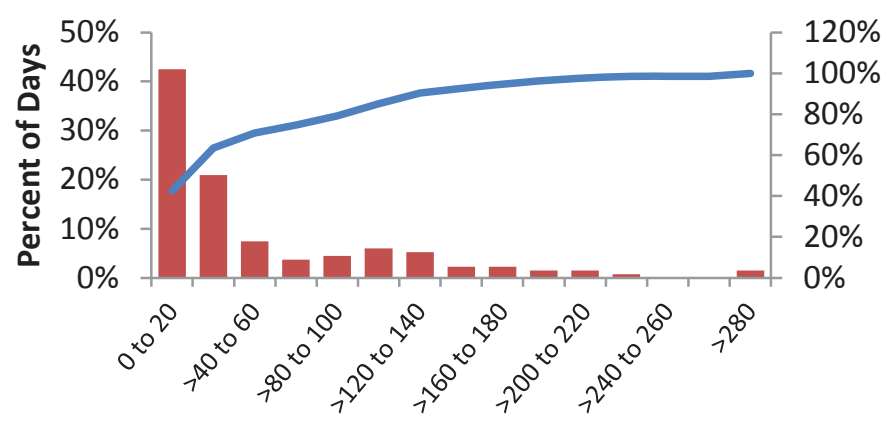

Daily Vehicle Trip Times (min)

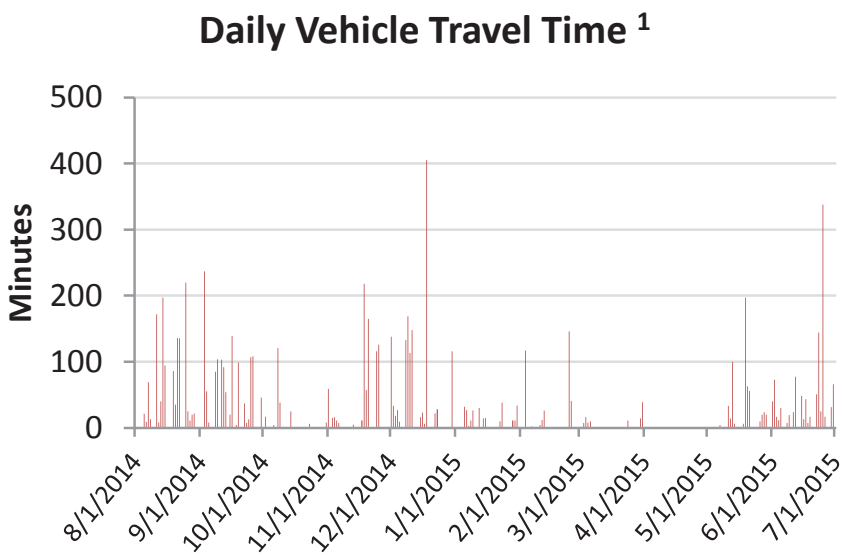

Note 1: Travel time includes all time when vehicle is in the "key on" state, even when the vehicle is not moving

$\begin{array}{cc}\text { Percentage of } & \text { Number of days } \\ \text { days vehicle } & \text { vehicle exceeds }\end{array}$

EV Range

59

63

67 exceeds EV range

$16 \%$

$15 \%$

$13 \%$
EV range

21

20

18 
Vehicle:

Report Period:

Model Year:

Vehicle Make:

Vehicle Model:

Body Type:
5M-G106212H

8/1/2014 00:00:00 - 7/1/2015 00:00:00

2009

Pontiac

G6

sedan
Total Number of Days with Driving

Average Number of Trips

Average Trip Distance

Total Number of Trips

Total Distance (miles)

Total Trip Duration (minutes)
162

4.4

15.3

711

2484.7

5419

\section{Distribution of Daily Vehicle Travel Distance}

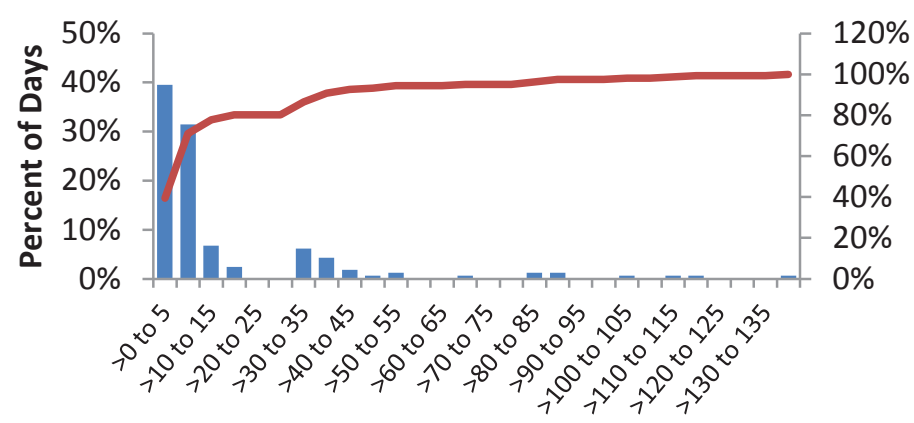

Daily Vehicle Miles Traveled

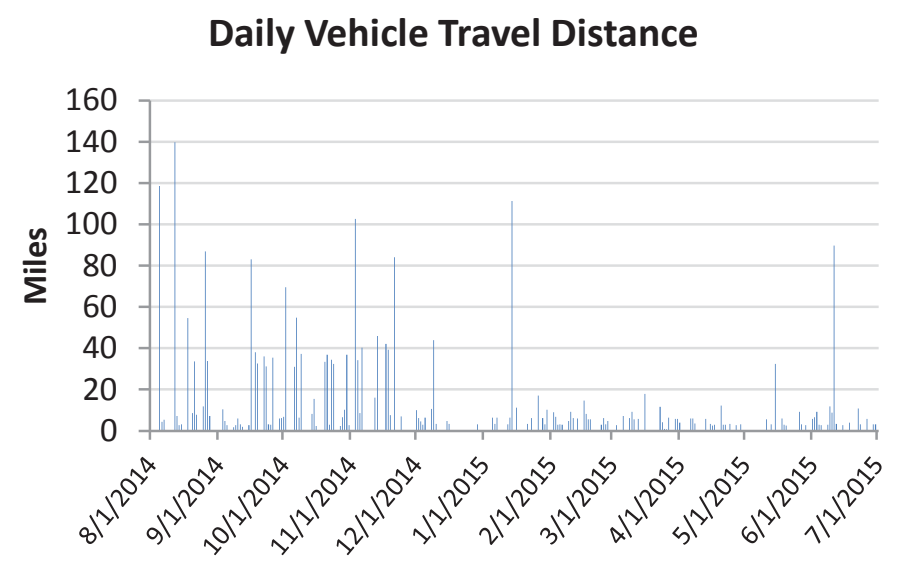

\section{Distribution of Daily Vehicle Travel Times ${ }^{1}$}

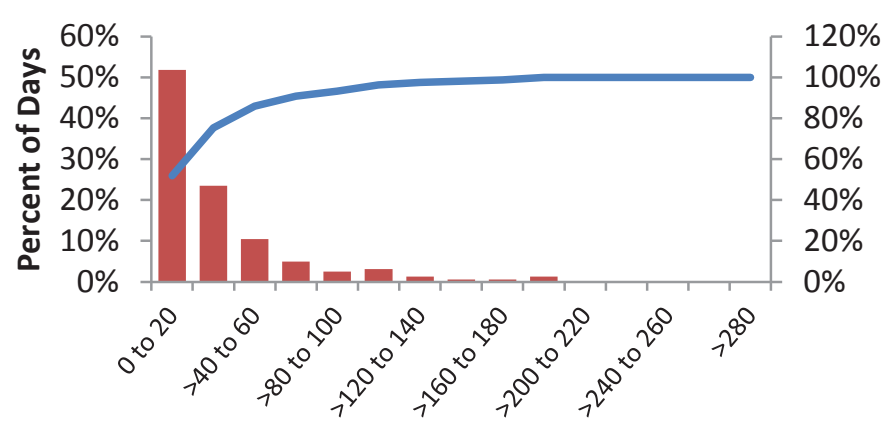

Daily Vehicle Trip Times (min)

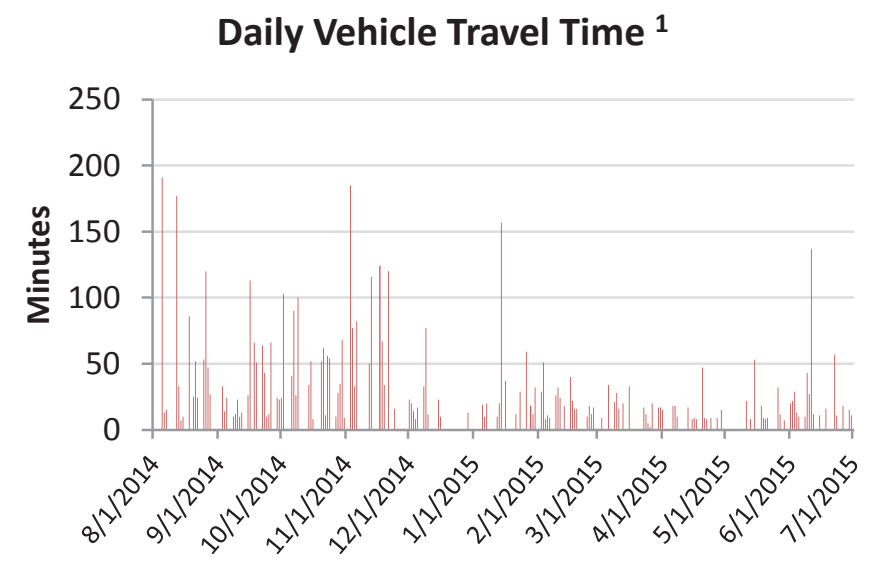

Note 1: Travel time includes all time when vehicle is in the "key on" state, even when the vehicle is not moving

$\begin{array}{cc}\text { Percentage of } & \text { Number of days } \\ \text { days vehicle } & \text { vehicle exceeds }\end{array}$

EV Range

59

63

67 exceeds EV range

$6 \%$

$6 \%$

$6 \%$
EV range

9

9 
Vehicle:

Report Period:

Model Year:

Vehicle Make:

Vehicle Model:

Body Type:
5M-G106209H

8/1/2014 00:00:00 - 7/1/2015 00:00:00

2009

Pontiac

G6

sedan
Total Number of Days with Driving Average Number of Trips

Average Trip Distance

Total Number of Trips

Total Distance (miles)

Total Trip Duration (minutes)
170

5.2

24.1

880

4099.6

8072

\section{Distribution of Daily Vehicle Travel Distance}

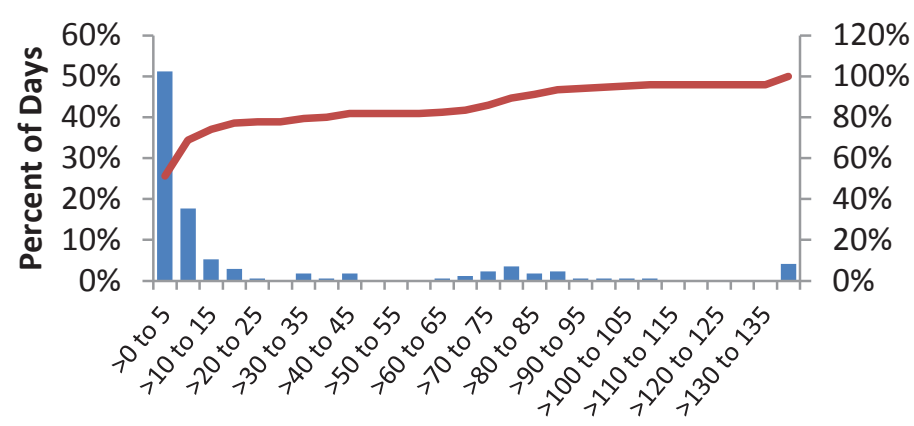

Daily Vehicle Miles Traveled

\section{Daily Vehicle Travel Distance}

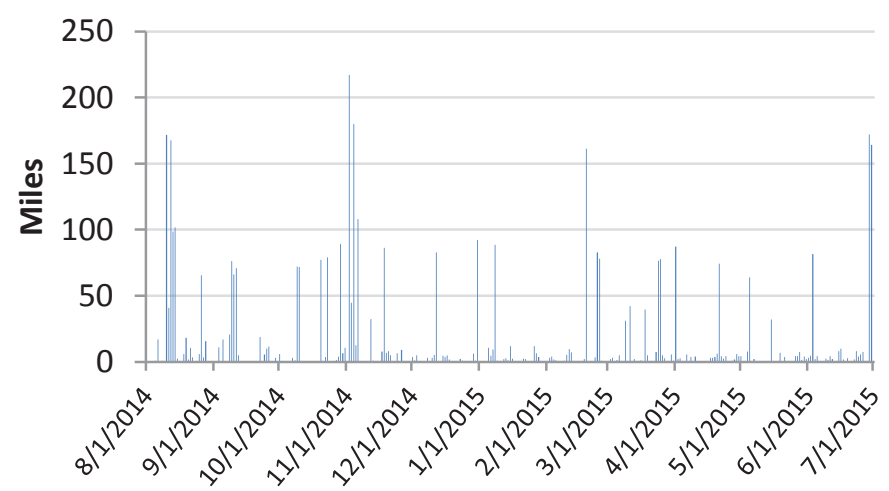

Distribution of Daily Vehicle Travel Times ${ }^{1}$

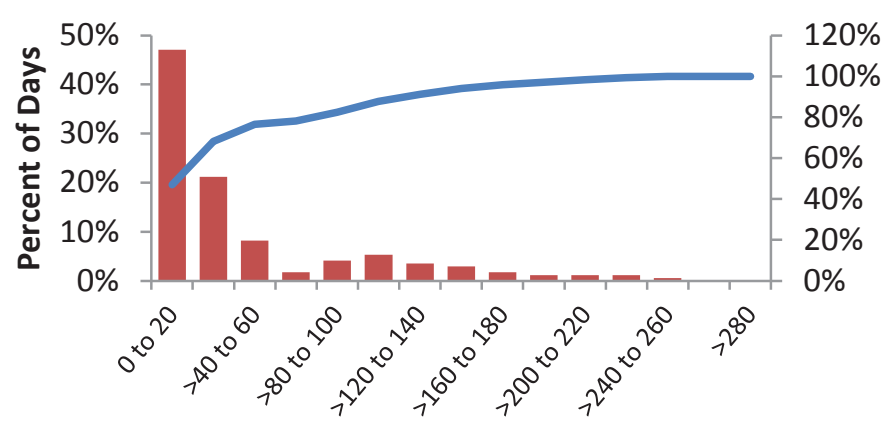

Daily Vehicle Trip Times (min)

\section{Daily Vehicle Travel Time ${ }^{1}$}

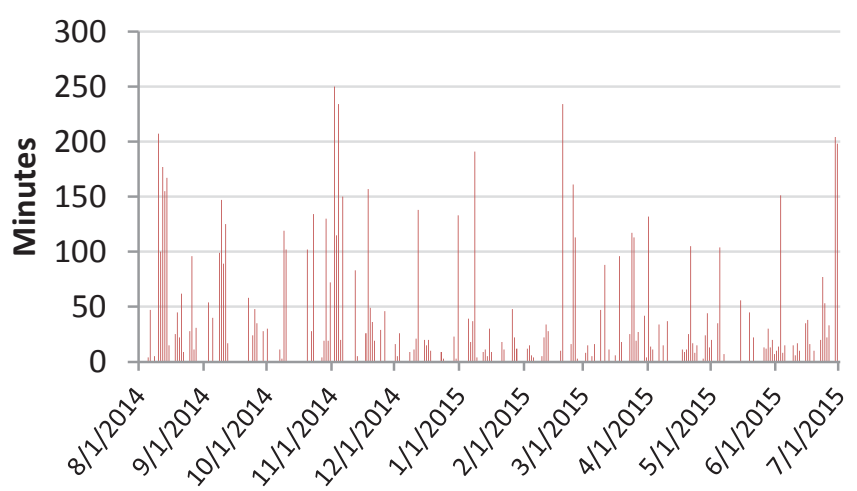

Note 1: Travel time includes all time when vehicle is in the "key on" state, even when the vehicle is not moving

Percentage of Number of days
days vehicle

EV Range

59

63

67 exceeds EV range

$18 \%$

$18 \%$

$16 \%$
EV range

31

31

28 
Vehicle:

Report Period:

Model Year:

Vehicle Make:

Vehicle Model:

Body Type:
5L-G422886H

8/1/2014 00:00:00 - 7/1/2015 00:00:00

2010

Chevrolet

Silverado

pickup

Total Number of Days with Driving

112

4.0

23.2

451

2598.8

6330

Total Trip Duration (minutes)

Distribution of Daily Vehicle Travel Times ${ }^{1}$

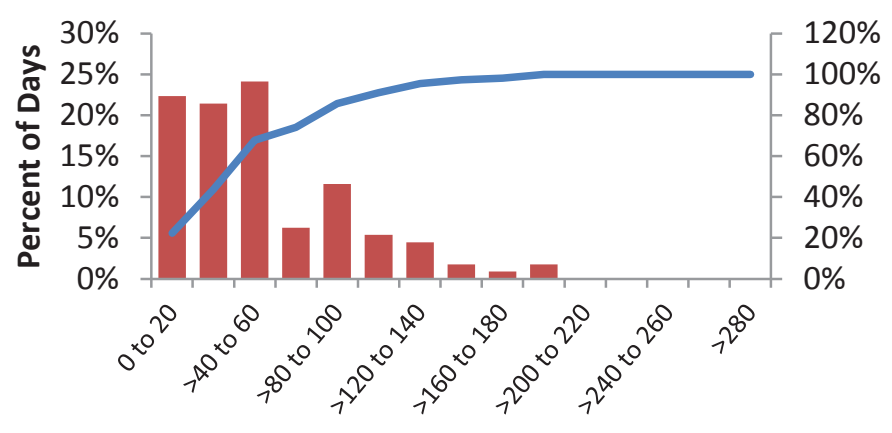

Daily Vehicle Trip Times (min)

Daily Vehicle Miles Traveled

\section{Daily Vehicle Travel Distance}

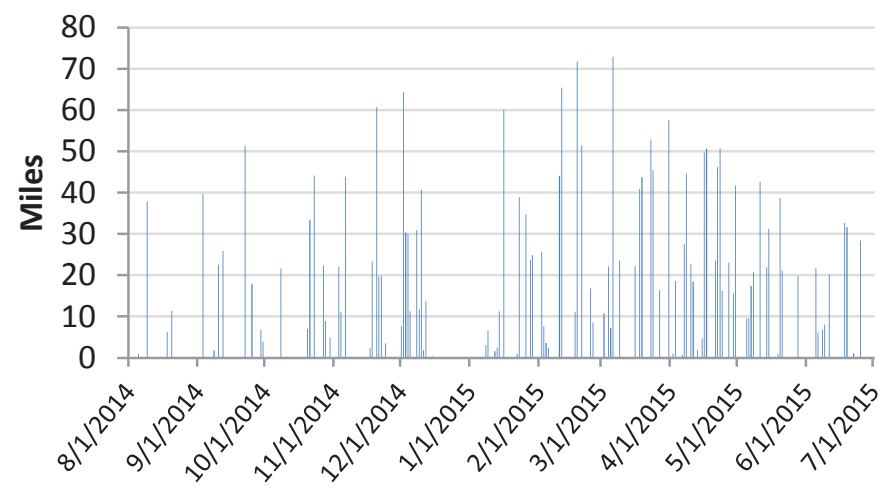

\section{Daily Vehicle Travel Time ${ }^{1}$}

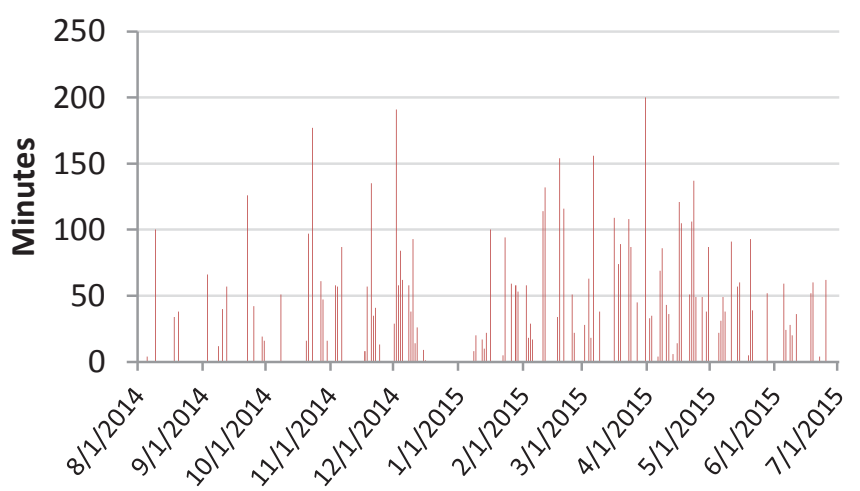

Note 1: Travel time includes all time when vehicle is in the "key on" state, even when the vehicle is not moving

$\begin{array}{cc}\text { Percentage of } & \text { Number of days } \\ \text { days vehicle } & \text { vehicle exceeds }\end{array}$

EV Range

59

63

67 exceeds EV range

$5 \%$

$4 \%$

$2 \%$
EV range

6

4

2 
Vehicle:

Report Period:

Model Year:

Vehicle Make:

Vehicle Model:

Body Type:
5L-G422884H

8/1/2014 00:00:00 - 7/1/2015 00:00:00

2010

Chevrolet

Silverado

pickup

Total Number of Days with Driving

Average Number of Trips

Average Trip Distance

Total Number of Trips

Total Distance (miles)

\section{Distribution of Daily Vehicle Travel Distance}

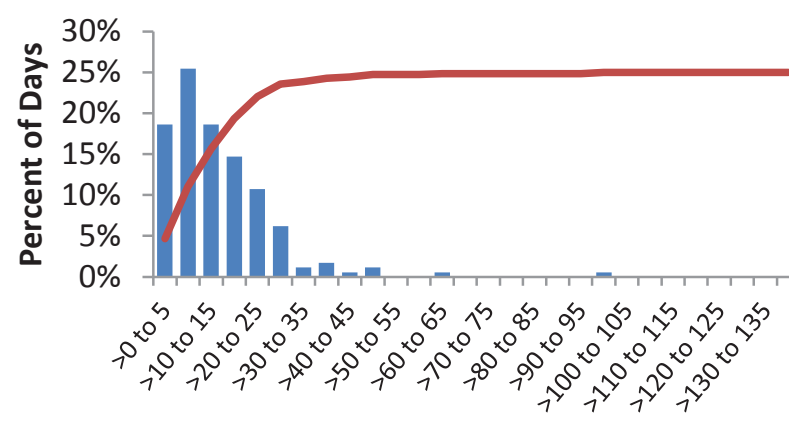

Daily Vehicle Miles Traveled

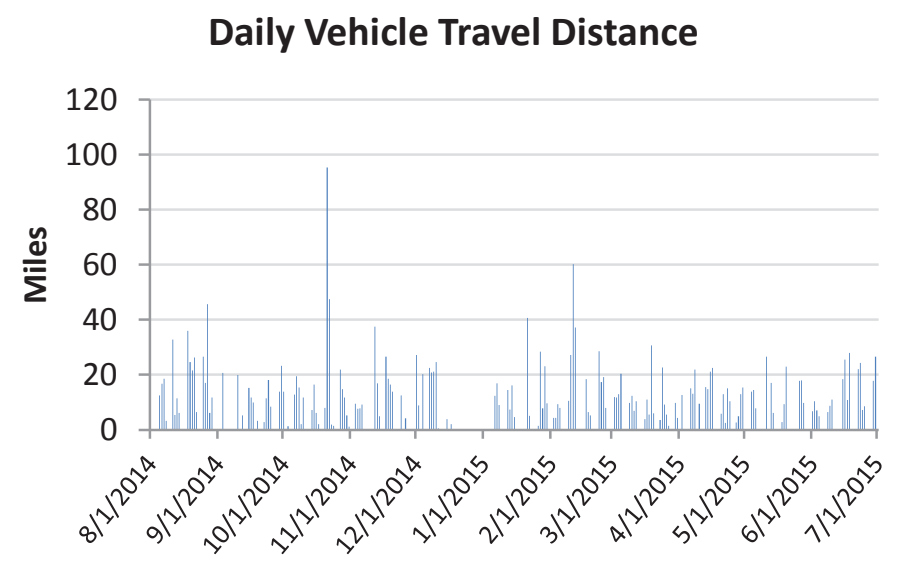

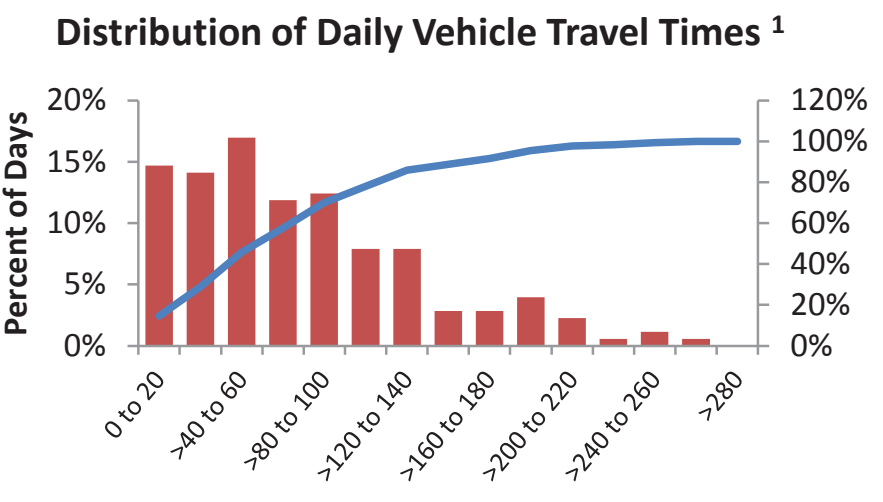

Daily Vehicle Trip Times (min)

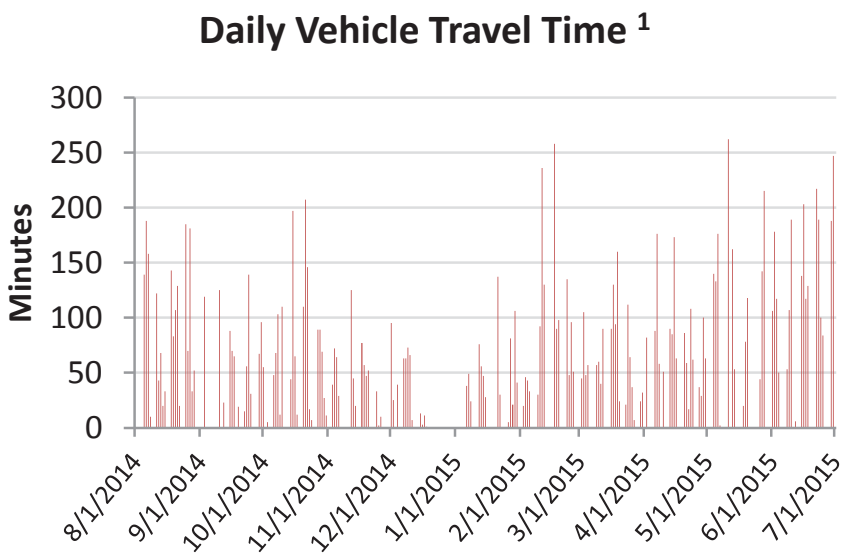

Note 1: Travel time includes all time when vehicle is in the "key on" state, even when the vehicle is not moving

$\begin{array}{ccc}\text { EV Range } & \begin{array}{c}\text { Percentage of } \\ \text { days vehicle } \\ \text { exceeds EV range }\end{array} & \begin{array}{c}\text { Number of days } \\ \text { vehicle exceeds } \\ \text { EV range }\end{array} \\ 63 & 1 \% & 2 \\ 67 & 1 \% & 1 \\ 63 & 1 \% & 1\end{array}$


Vehicle:

Report Period:

Model Year:

Vehicle Make:

Vehicle Model:

Body Type:
5L-G422785H

8/1/2014 00:00:00 - 7/1/2015 00:00:00

2010

Chevrolet

Silverado

pickup

Total Number of Days with Driving

Average Number of Trips

6.3

Average Trip Distance

Total Number of Trips

Total Distance (miles)

7015.5

Total Trip Duration (minutes)

15571

\section{Distribution of Daily Vehicle Travel Distance}

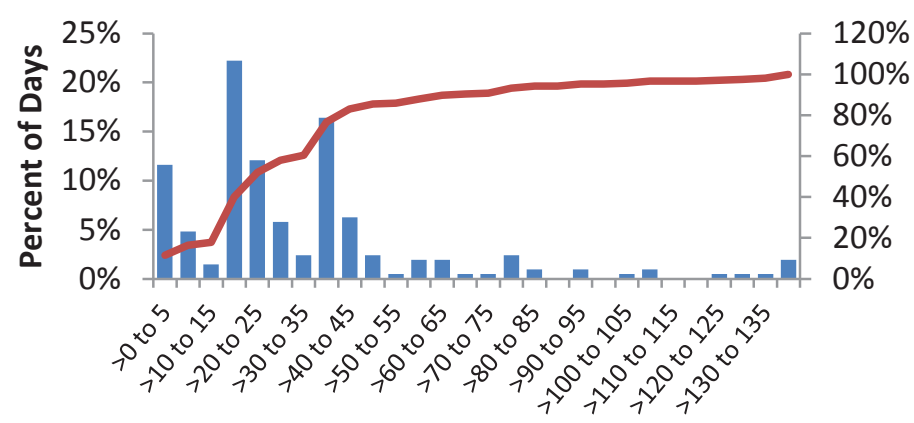

Daily Vehicle Miles Traveled

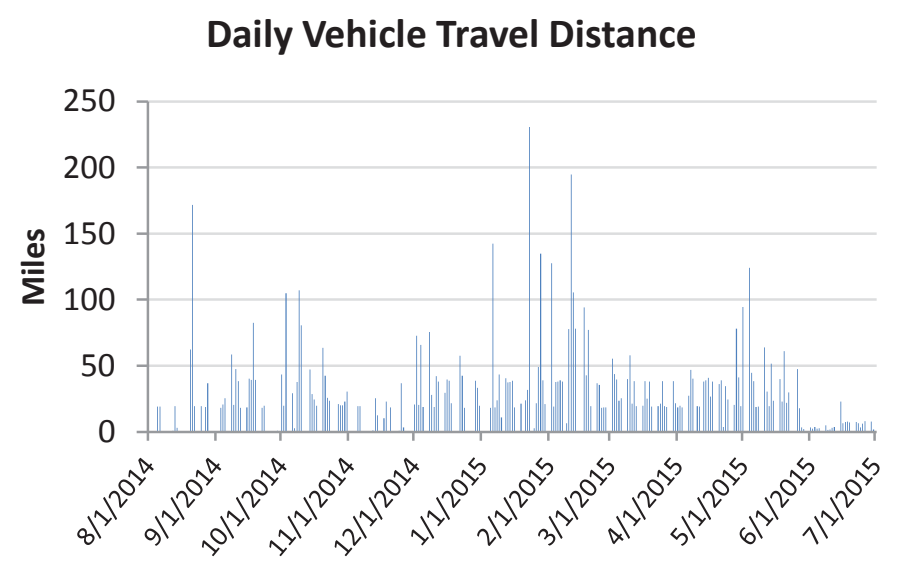

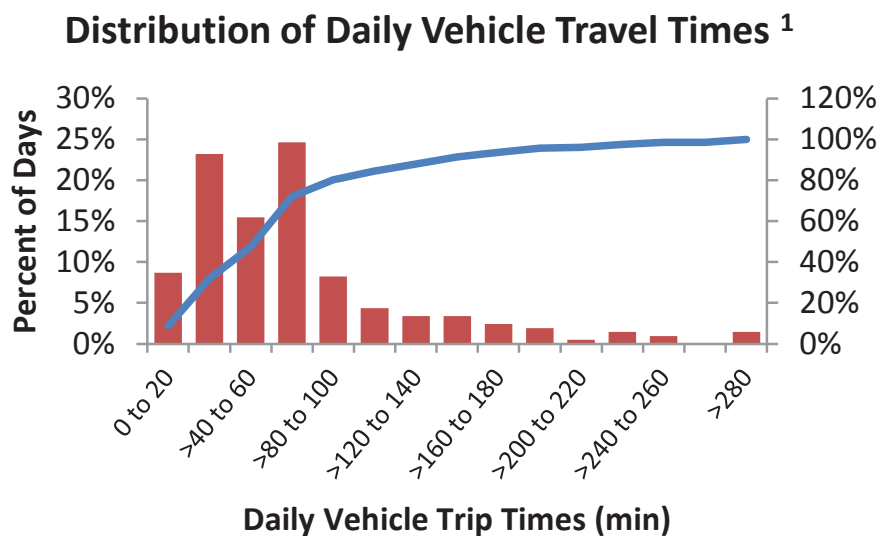

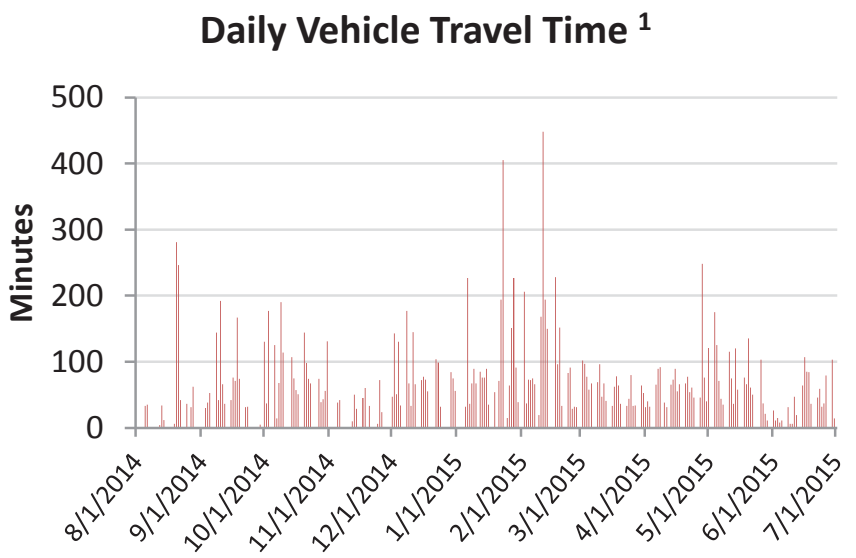

Note 1: Travel time includes all time when vehicle is in the "key on" state, even when the vehicle is not moving

$\begin{array}{ccc}\text { EV Range } & \begin{array}{c}\text { Percentage of } \\ \text { days vehicle } \\ \text { exceeds EV range }\end{array} & \begin{array}{c}\text { Number of days } \\ \text { vehicle exceeds } \\ \text { EV range }\end{array} \\ 69 & 12 \% & 25 \\ 63 & 11 \% & 23 \\ 67 & 10 \% & 20\end{array}$


Vehicle:

Report Period:

Model Year:

Vehicle Make:

Vehicle Model:

Body Type:
5L-G422486K

8/1/2014 00:00:00 - 7/1/2015 00:00:00

2010

Chevrolet

Express

van

Total Number of Days with Driving

183

5.2

19.0

950

3482.7

11290

Total Distance (miles)
Total Trip Duration (minutes)

\section{Distribution of Daily Vehicle Travel Times ${ }^{1}$}

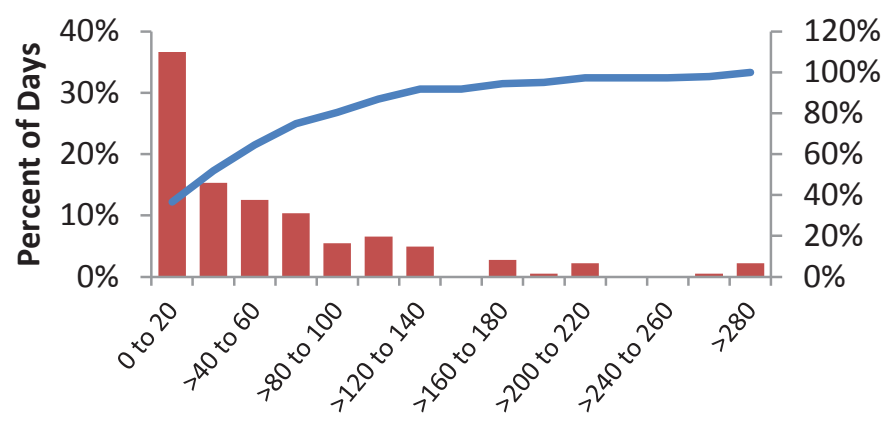

Daily Vehicle Trip Times (min)

Daily Vehicle Miles Traveled

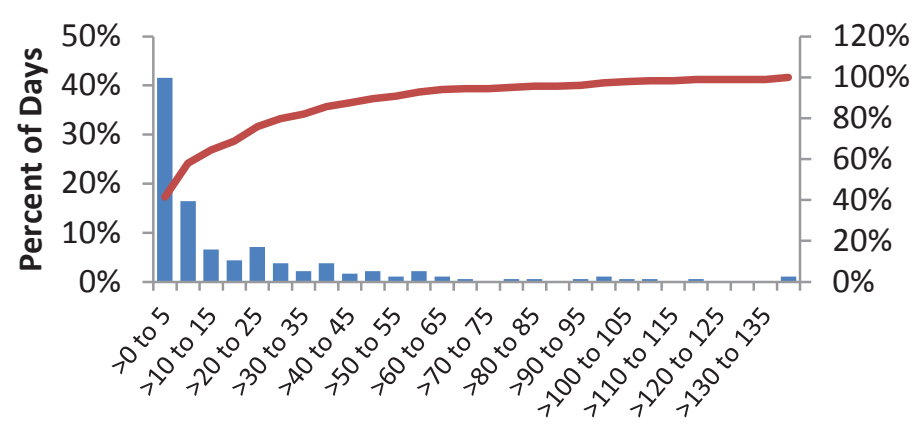

\section{Daily Vehicle Travel Distance}

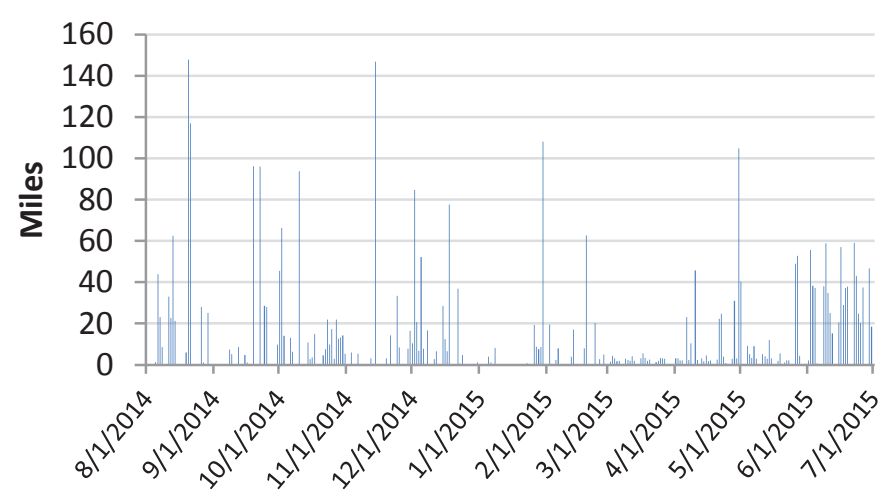

\section{Daily Vehicle Travel Time ${ }^{1}$}

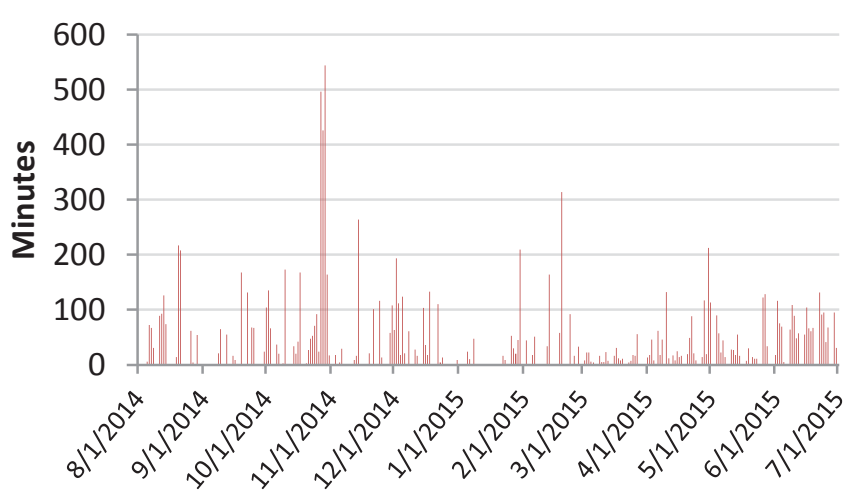

Note 1: Travel time includes all time when vehicle is in the "key on" state, even when the vehicle is not moving

$\begin{array}{cc}\text { Percentage of } & \text { Number of days } \\ \text { days vehicle } & \text { vehicle exceeds }\end{array}$

EV Range

59

63

67 exceeds EV range

$7 \%$

$6 \%$

$5 \%$
EV range

13

11

10 
Vehicle:

Report Period:

Model Year:

Vehicle Make:

Vehicle Model:

Body Type:
5L-G422482K

8/1/2014 00:00:00 - 7/1/2015 00:00:00

2010

Chevrolet

Express

van

Total Number of Days with Driving

150

5.2

71.7

783

10756.5

22992

Total Trip Duration (minutes)

\section{Distribution of Daily Vehicle Travel Times ${ }^{1}$}

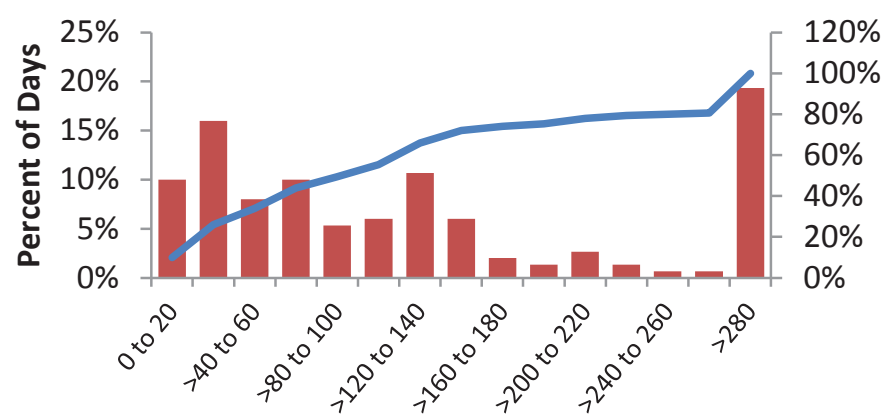

Daily Vehicle Miles Traveled

\section{Daily Vehicle Travel Distance}

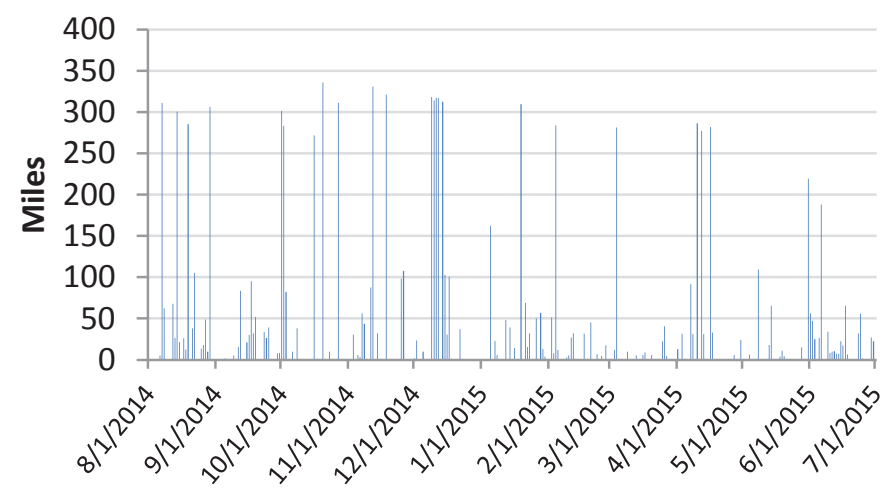

Daily Vehicle Trip Times (min)

$120 \%$

$100 \%$

$80 \%$

$60 \%$

$40 \%$

$20 \%$

$0 \%$

\section{Daily Vehicle Travel Time ${ }^{1}$}

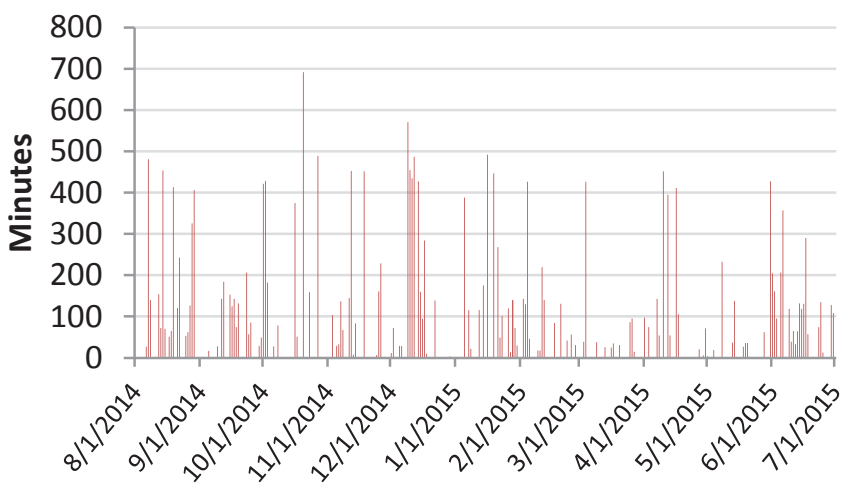

Note 1: Travel time includes all time when vehicle is in the "key on" state, even when the vehicle is not moving

$\begin{array}{cc}\text { Percentage of } & \text { Number of days } \\ \text { days vehicle } & \text { vehicle exceeds }\end{array}$

EV Range

59

63

67 exceeds EV range

$27 \%$

$27 \%$

$25 \%$
EV range

41

40

38 
Vehicle:

Report Period:

Model Year:

Vehicle Make:

Vehicle Model:

Body Type:
5L-G422467K

8/1/2014 00:00:00 - 7/1/2015 00:00:00

2010

Chevrolet

Silverado 1500

pickup
Total Number of Days with Driving Average Number of Trips

Average Trip Distance

Total Number of Trips

Total Distance (miles)

Total Trip Duration (minutes)
200

7.4

26.4

1477

5278.8

11852

\section{Distribution of Daily Vehicle Travel Distance}

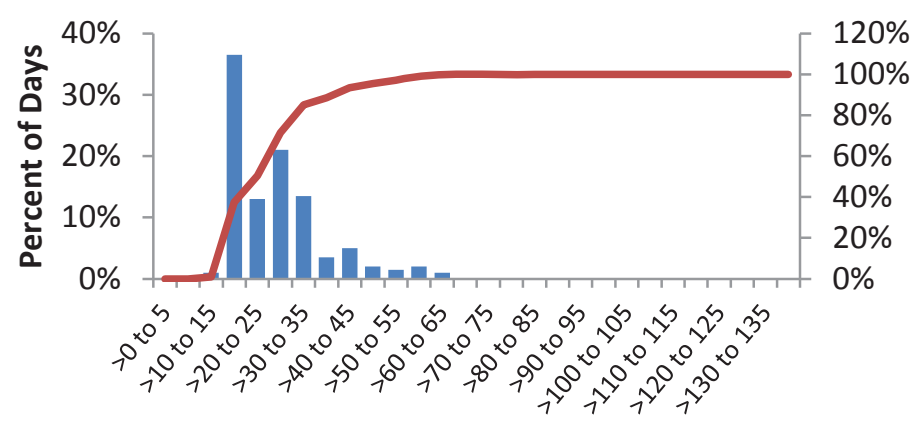

Daily Vehicle Miles Traveled

\section{Daily Vehicle Travel Distance}

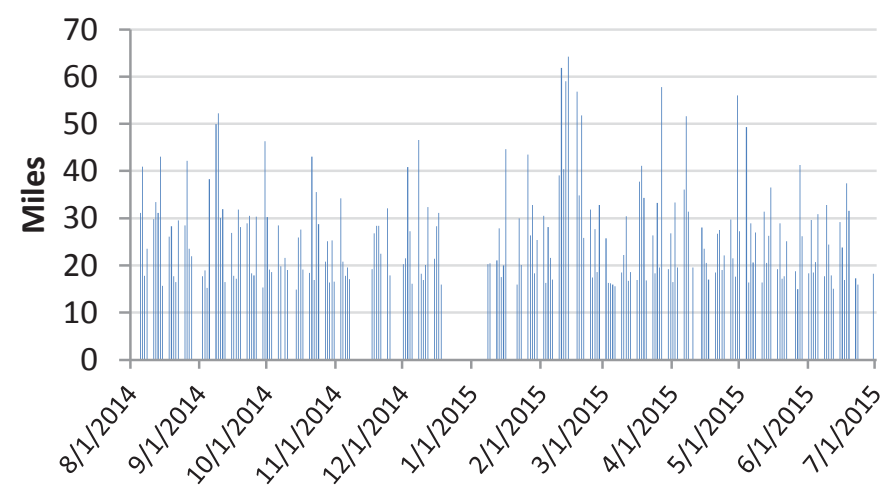

Distribution of Daily Vehicle Travel Times ${ }^{1}$

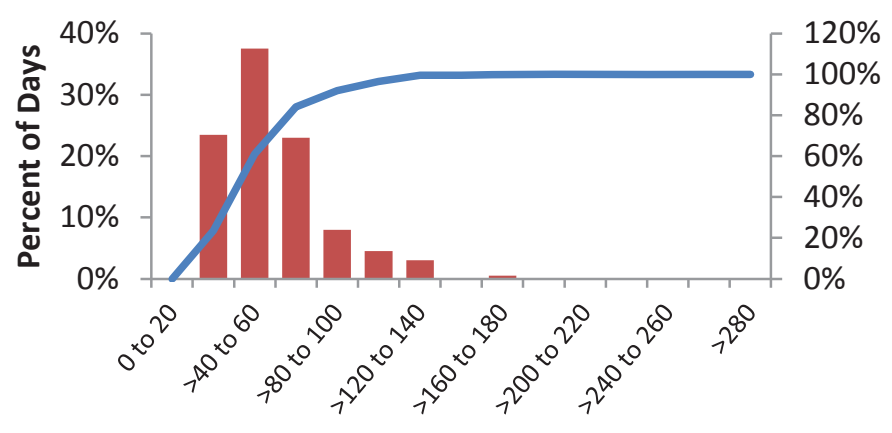

Daily Vehicle Trip Times (min)

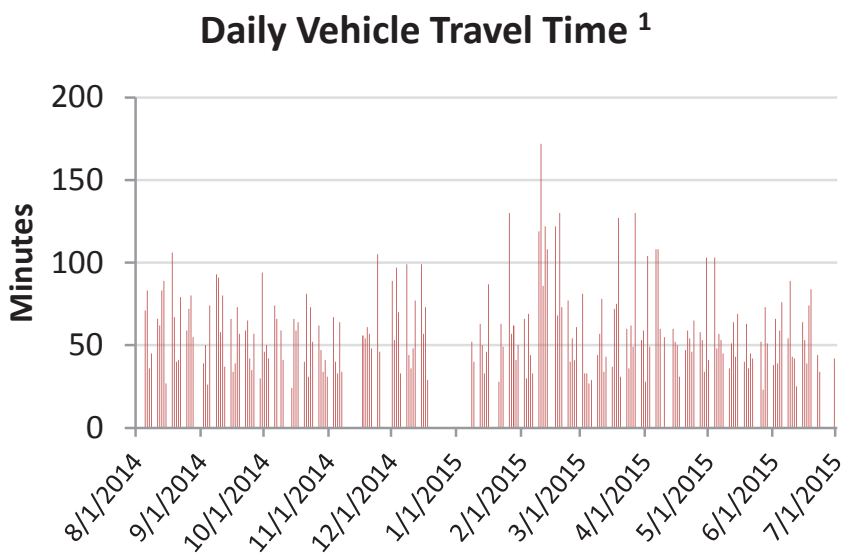

Note 1: Travel time includes all time when vehicle is in the "key on" state, even when the vehicle is not moving

$\begin{array}{cc}\text { Percentage of } & \text { Number of days } \\ \text { days vehicle } & \text { vehicle exceeds }\end{array}$

EV Range

59

63

67 exceeds EV range

$1 \%$

$0 \%$

$0 \%$
EV range

2

1

0 
Vehicle:

Report Period:

Model Year:

Vehicle Make:

Vehicle Model:

Body Type:
5L-G422235H

8/1/2014 00:00:00 - 7/1/2015 00:00:00

2009

FORD

F-150

pickup

Total Number of Days with Driving

160

7.3

25.5

1160

4080.5

11925

Total Distance (miles)
Total Trip Duration (minutes)

Distribution of Daily Vehicle Travel Times ${ }^{1}$

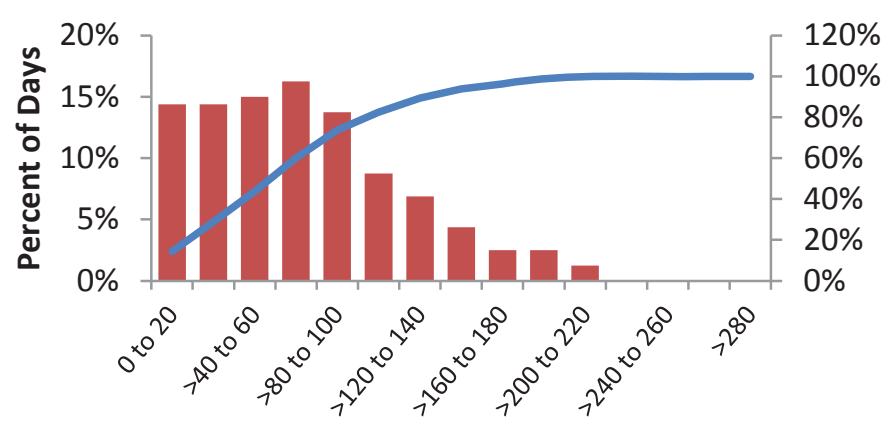

Daily Vehicle Trip Times (min)

Daily Vehicle Miles Traveled

$120 \%$

$100 \%$

$60 \%$

$40 \%$

$20 \%$

$0 \%$

\section{Daily Vehicle Travel Distance}

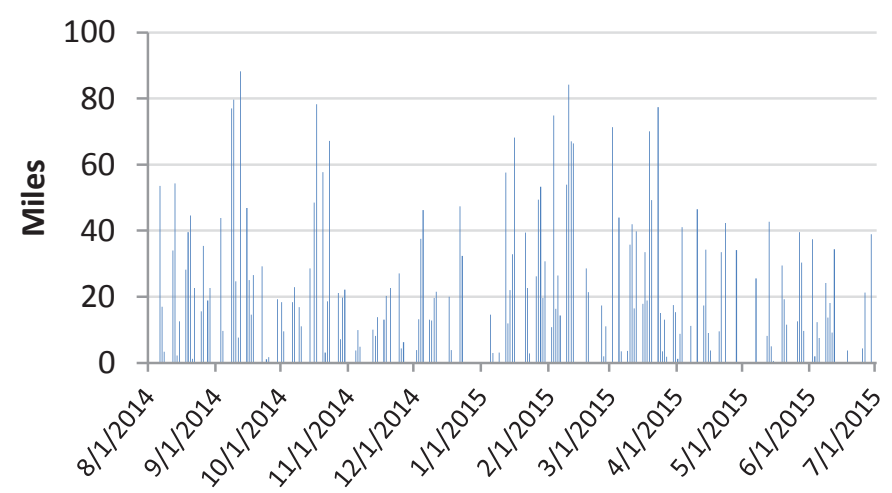

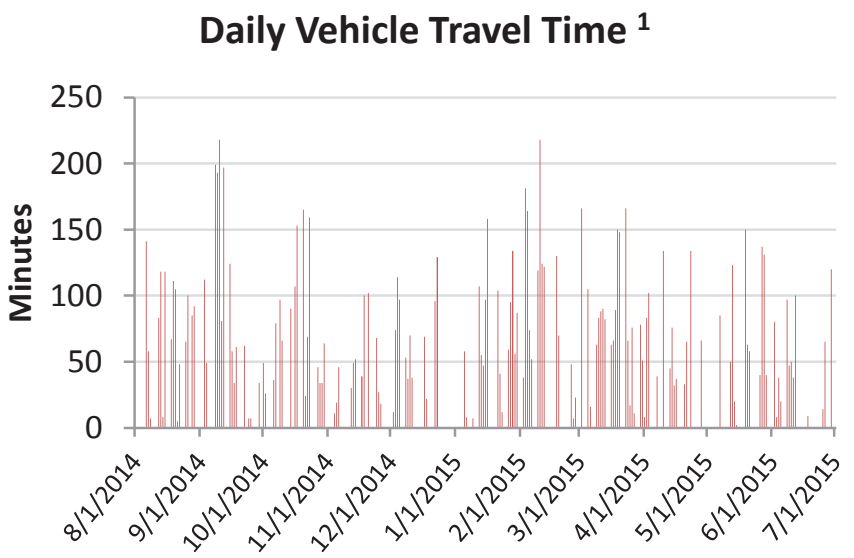

Note 1: Travel time includes all time when vehicle is in the "key on" state, even when the vehicle is not moving

$\begin{array}{cc}\text { Percentage of } & \text { Number of days } \\ \text { days vehicle } & \text { vehicle exceeds }\end{array}$

EV Range

59

63

67 exceeds EV range

$8 \%$

$8 \%$

$7 \%$
EV range

13

13

11 
Vehicle:

Report Period:

Model Year:

Vehicle Make:

Vehicle Model:

Body Type:
5L-G422233H

8/1/2014 00:00:00 - 7/1/2015 00:00:00

2009

FORD

F-150

pickup
Total Number of Days with Driving Average Number of Trips

Average Trip Distance

Total Number of Trips

Total Distance (miles)

Total Trip Duration (minutes)
150

5.1

24.2

769

3625.5

8562

\section{Distribution of Daily Vehicle Travel Distance}

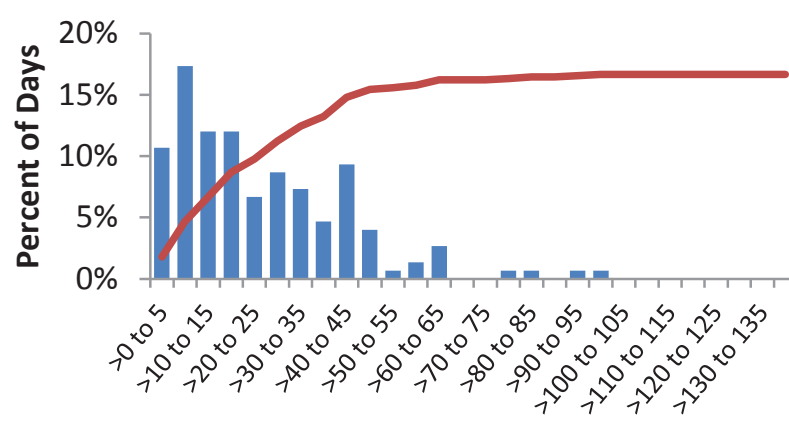

Daily Vehicle Miles Traveled

\section{Daily Vehicle Travel Distance}

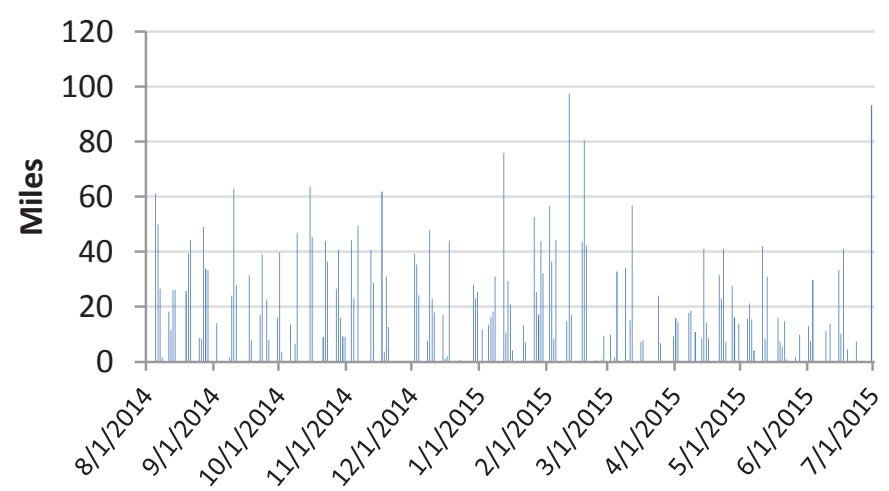

\section{Distribution of Daily Vehicle Travel Times ${ }^{1}$}

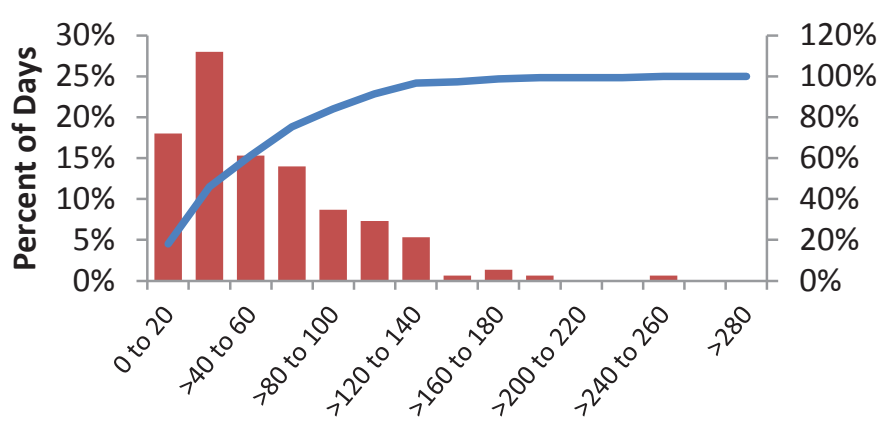

Daily Vehicle Trip Times (min)

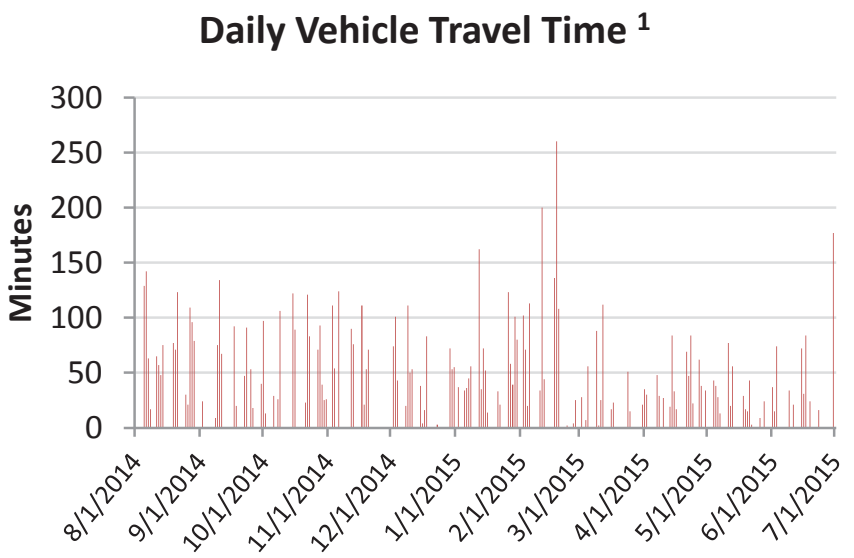

Note 1: Travel time includes all time when vehicle is in the "key on" state, even when the vehicle is not moving

$\begin{array}{cc}\text { Percentage of } & \text { Number of days } \\ \text { days vehicle } & \text { vehicle exceeds }\end{array}$

EV Range

59

63

67

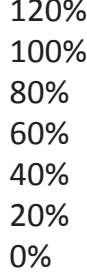

$100 \%$

$60 \%$

$40 \%$

$20 \%$

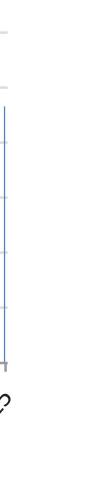


Vehicle:

Report Period:

Model Year:

Vehicle Make:

Vehicle Model:

Body Type:
5L-G422227H

8/1/2014 00:00:00 - 7/1/2015 00:00:00

2009

FORD

F-150

pickup

Total Number of Days with Driving

149

4.6

58.5

685

8711.3

21202

Total Trip Duration (minutes)

\section{Distribution of Daily Vehicle Travel Times ${ }^{1}$}

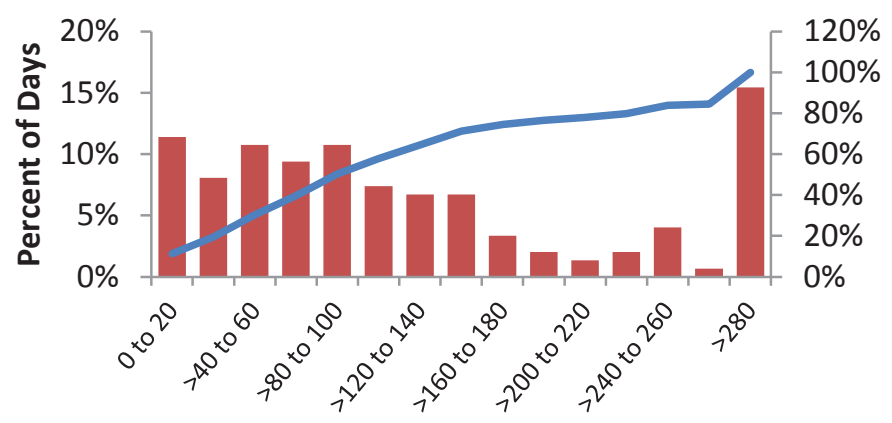

Daily Vehicle Miles Traveled

\section{Daily Vehicle Travel Distance}

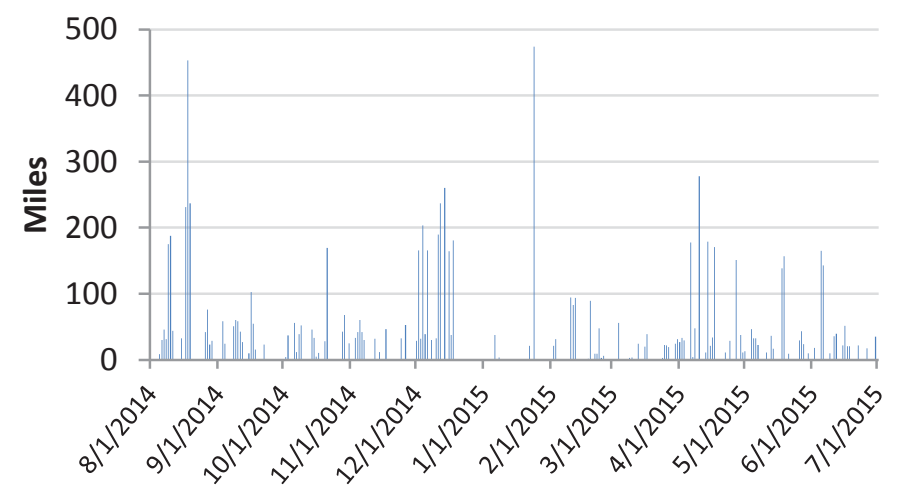

Daily Vehicle Trip Times (min)

$120 \%$

$100 \%$

$80 \%$

$60 \%$

$40 \%$

$20 \%$

$0 \%$

\section{Daily Vehicle Travel Time ${ }^{1}$}

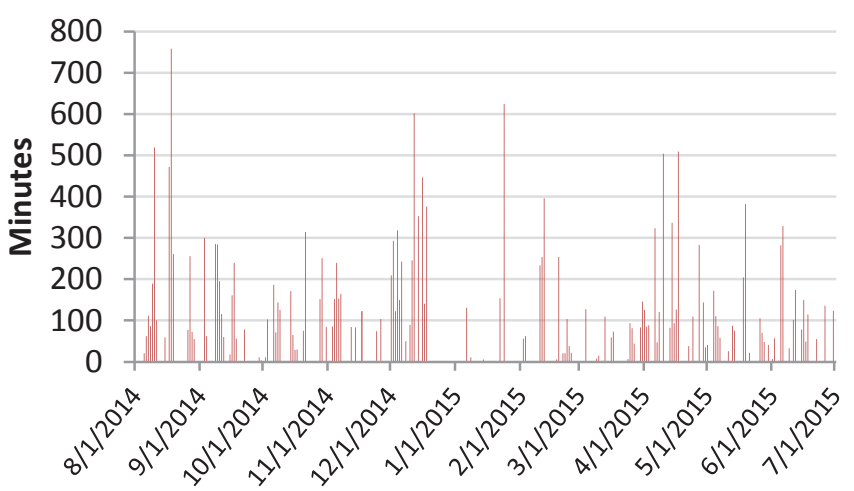

Note 1: Travel time includes all time when vehicle is in the "key on" state, even when the vehicle is not moving

Percentage of Number of days
days vehicle

EV Range

59

63

67 exceeds EV range

$22 \%$

$21 \%$

$21 \%$
EV range

33

31

31 
Vehicle:

Report Period:

Model Year:

Vehicle Make:

Vehicle Model:

Body Type:
5L-G422215H

8/1/2014 00:00:00 - 7/1/2015 00:00:00

2009

Chevrolet

Express

van

Total Number of Days with Driving

Average Number of Trips

Average Trip Distance

Total Number of Trips

1859

Total Distance (miles)

Total Trip Duration (minutes)

48249

\section{Distribution of Daily Vehicle Travel Distance}

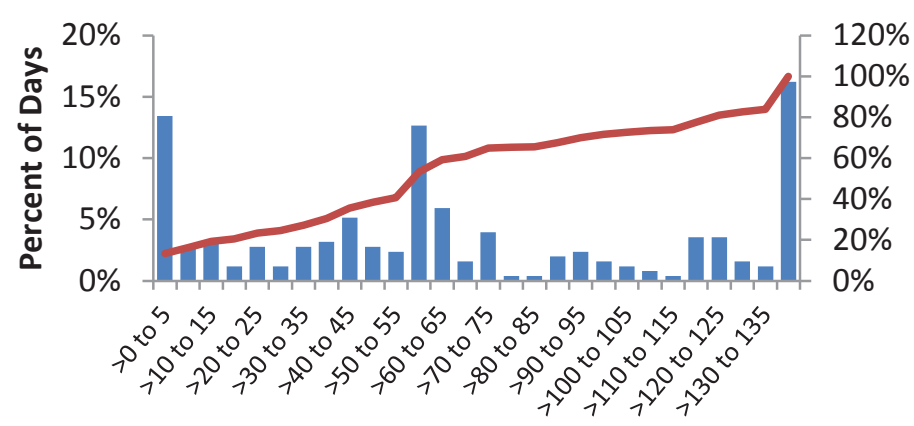

Daily Vehicle Miles Traveled

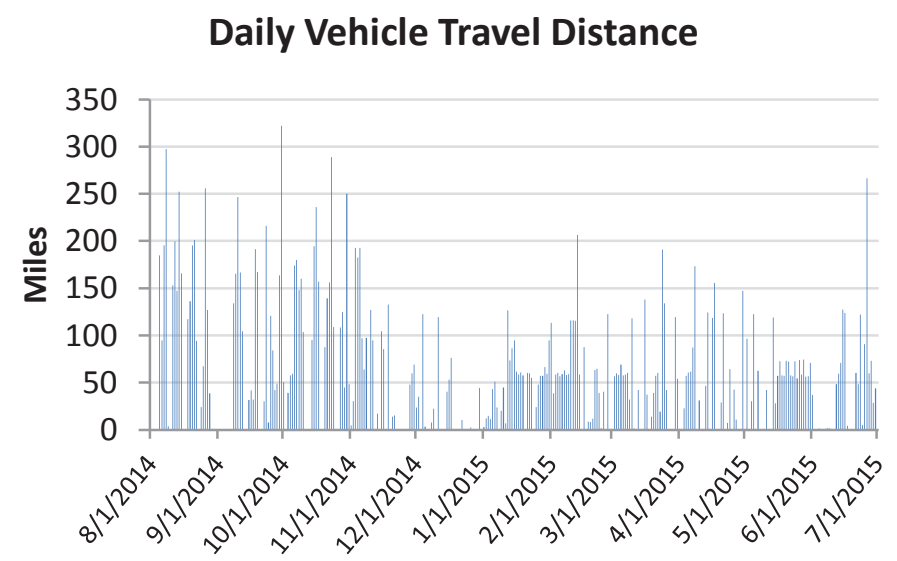

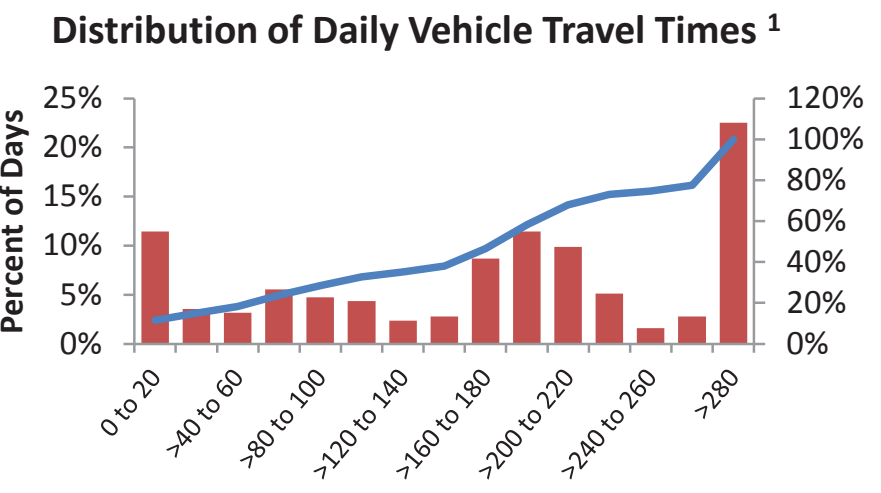

Daily Vehicle Trip Times (min)

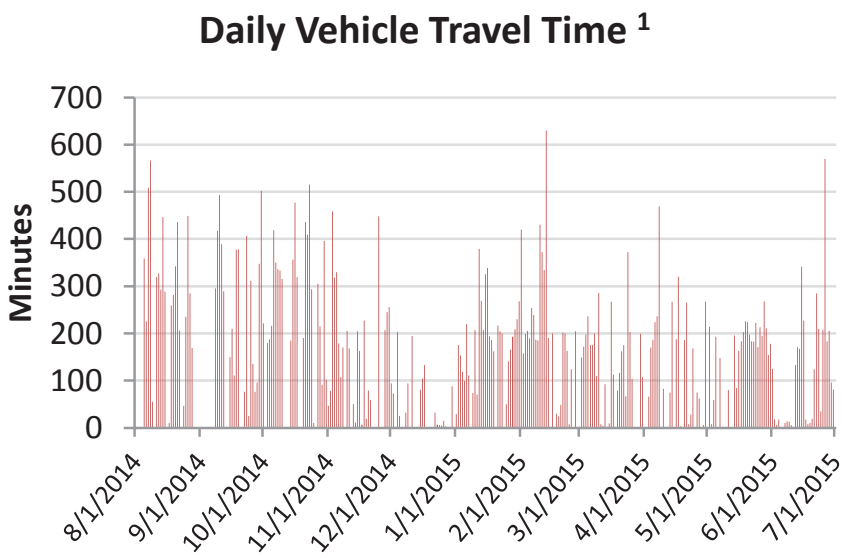

Note 1: Travel time includes all time when vehicle is in the "key on" state, even when the vehicle is not moving

$\begin{array}{ccc}\text { EV Range } & \begin{array}{c}\text { Percentage of } \\ \text { days vehicle } \\ \text { exceeds EV range }\end{array} & \begin{array}{c}\text { Number of days } \\ \text { vehicle exceeds } \\ \text { EV range }\end{array} \\ 63 & 49 \% & 125 \\ 67 & 42 \% & 107 \\ & 40 \% & 102\end{array}$


Vehicle:

Report Period:

Model Year:

Vehicle Make:

Vehicle Model:

Body Type:
5L-G422206H

8/1/2014 00:00:00 - 7/1/2015 00:00:00

2009

Chevrolet

Express 1500

van
Total Number of Days with Driving

Average Number of Trips

Average Trip Distance

Total Number of Trips

Total Distance (miles)

Total Trip Duration (minutes)
123

5.5

49.0

682

6021.3

14973

\section{Distribution of Daily Vehicle Travel Distance}

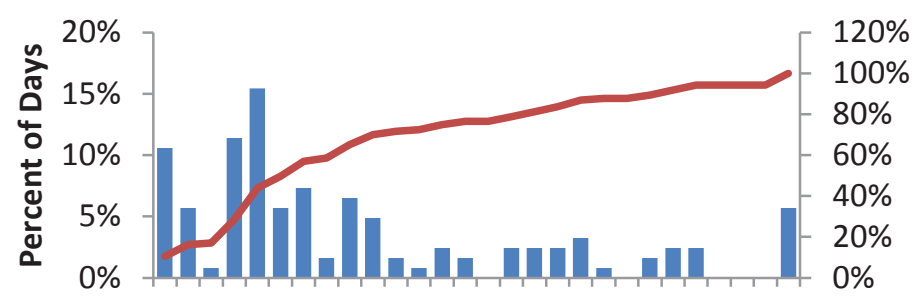

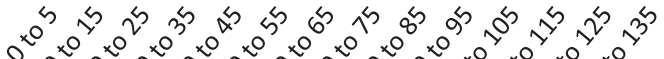

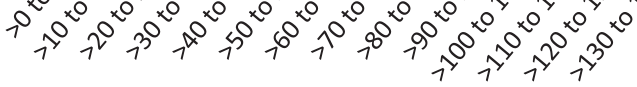

Daily Vehicle Miles Traveled

\section{Daily Vehicle Travel Distance}

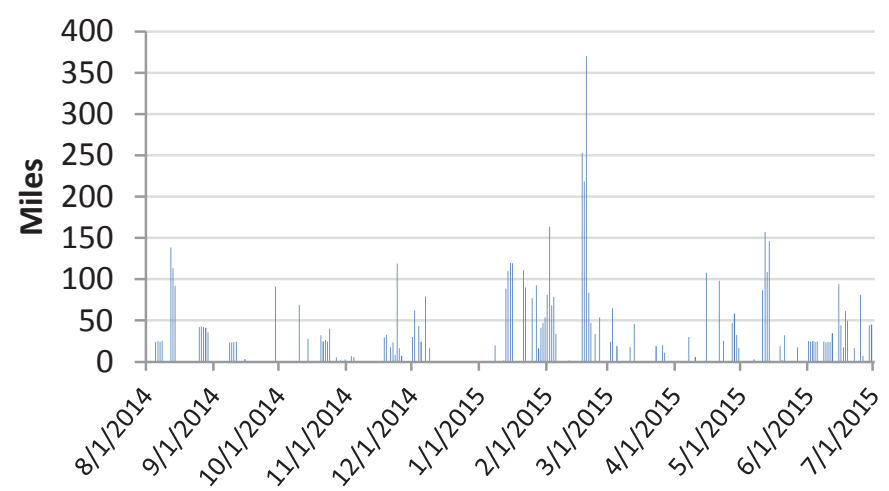

Distribution of Daily Vehicle Travel Times ${ }^{1}$

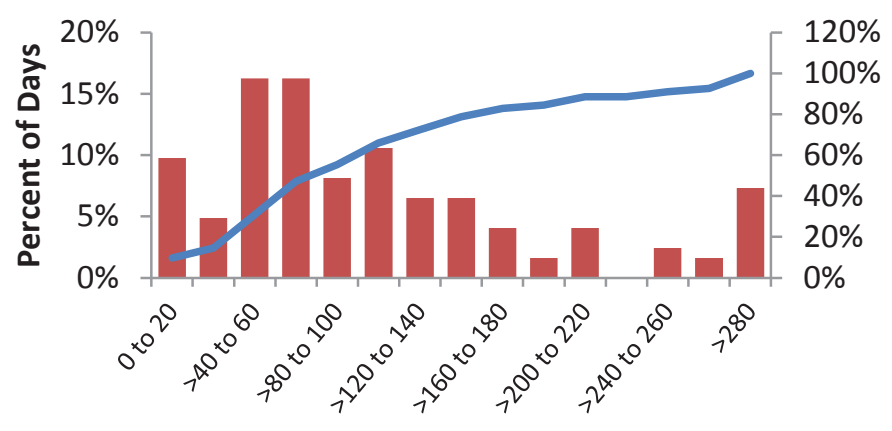

Daily Vehicle Trip Times (min)

\section{Daily Vehicle Travel Time ${ }^{1}$}

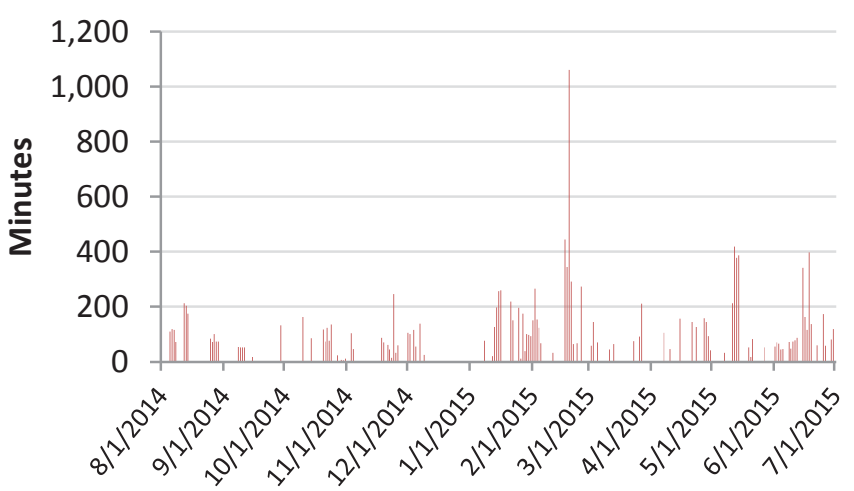

Note 1: Travel time includes all time when vehicle is in the "key on" state, even when the vehicle is not moving

$\begin{array}{cc}\text { Percentage of } & \text { Number of days } \\ \text { days vehicle } & \text { vehicle exceeds }\end{array}$

EV Range

59

63

67 exceeds EV range

$28 \%$

$26 \%$

$25 \%$
EV range

34

32

31 
Vehicle:

Report Period:

Model Year:

Vehicle Make:

Vehicle Model:

Body Type:
5L-G422169G

8/1/2014 00:00:00 - 7/1/2015 00:00:00

2008

Chevrolet

Express

van

Total Number of Days with Driving

126

3.5

68.5

436

8637.0

15037

\section{Distribution of Daily Vehicle Travel Times ${ }^{1}$}

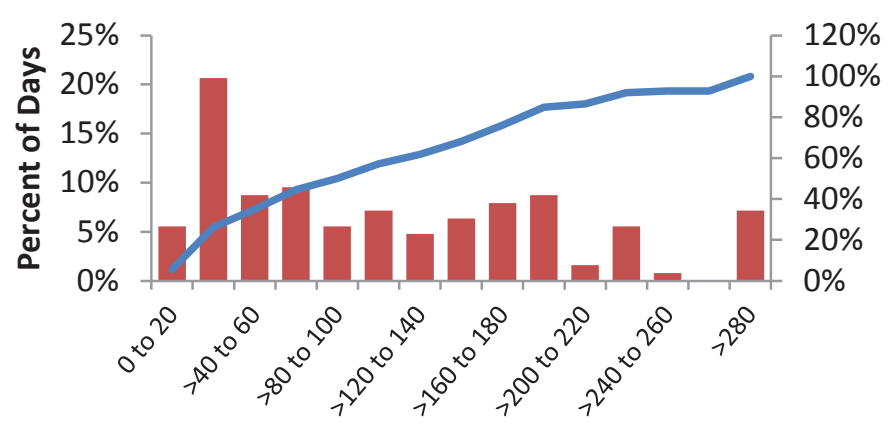

Daily Vehicle Trip Times (min)

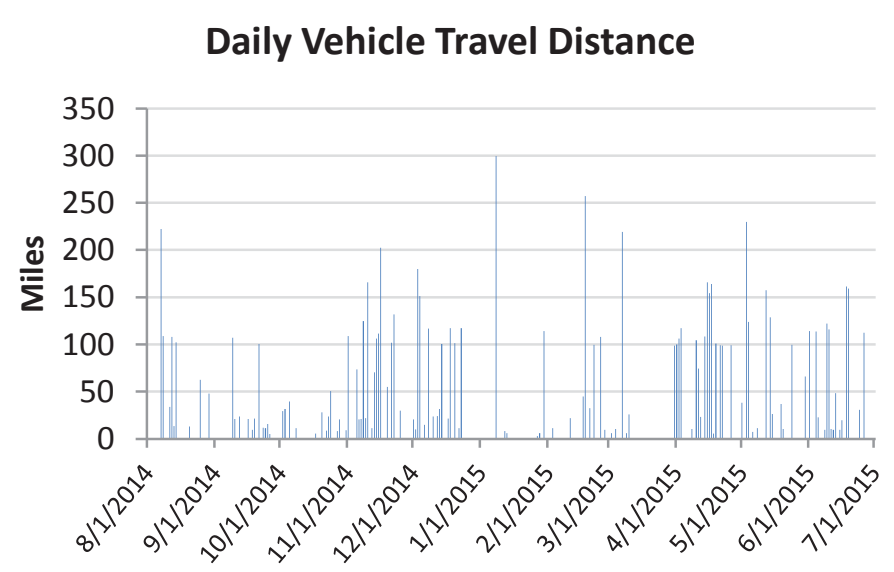

Daily Vehicle Miles Traveled

\section{Daily Vehicle Travel Distance}

$120 \%$

$100 \%$

$80 \%$

$60 \%$

$40 \%$

$20 \%$

$0 \%$

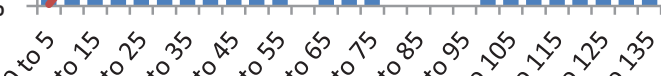

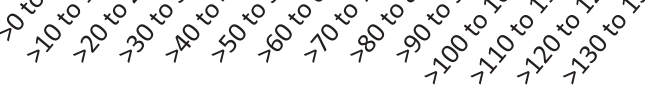

\section{Daily Vehicle Travel Time ${ }^{1}$}

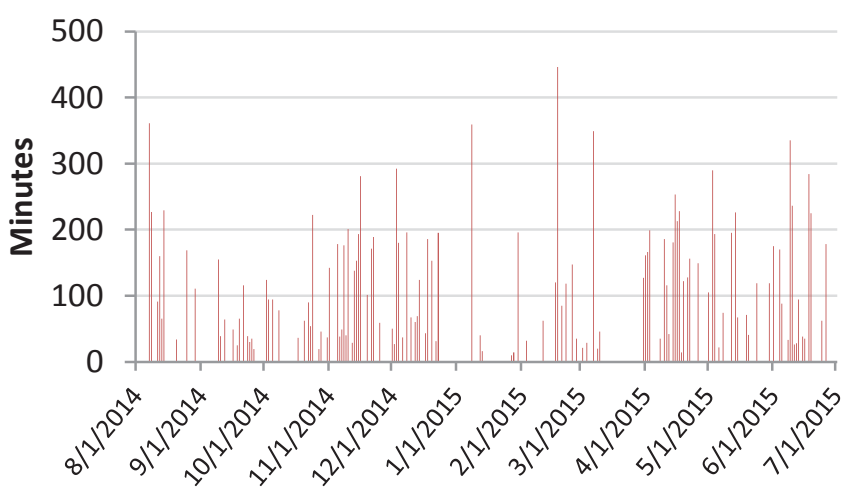

Note 1: Travel time includes all time when vehicle is in the "key on" state, even when the vehicle is not moving

$\begin{array}{cc}\text { Percentage of } & \text { Number of days } \\ \text { days vehicle } & \text { vehicle exceeds }\end{array}$

EV Range

59

63

67 exceeds EV range

$45 \%$

$44 \%$

$44 \%$
EV range

57

56

55 
Vehicle:

Report Period:

Model Year:

Vehicle Make:

Vehicle Model:

Body Type:
5L-G421922L

8/1/2014 00:00:00 - 7/1/2015 00:00:00

2011

FORD

F-150

pickup
Total Number of Days with Driving Average Number of Trips

Average Trip Distance

Total Number of Trips

Total Distance (miles)

Total Trip Duration (minutes)
60

3.7

34.7

220

2080.8

6059

\section{Distribution of Daily Vehicle Travel Distance}

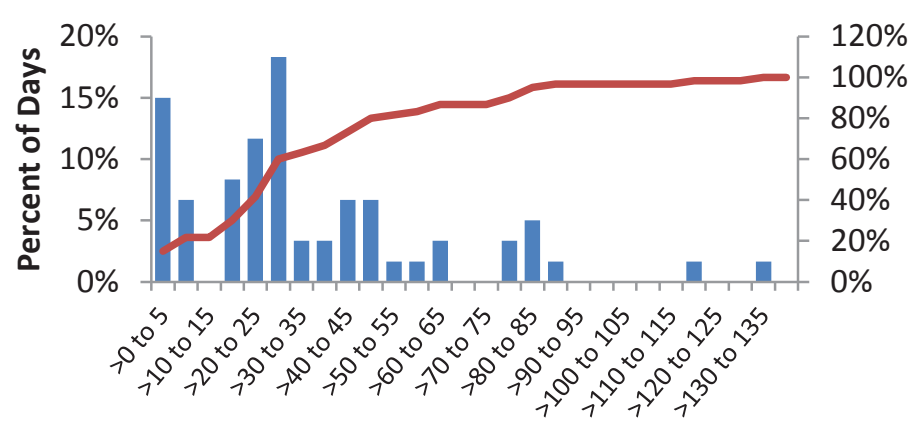

Daily Vehicle Miles Traveled

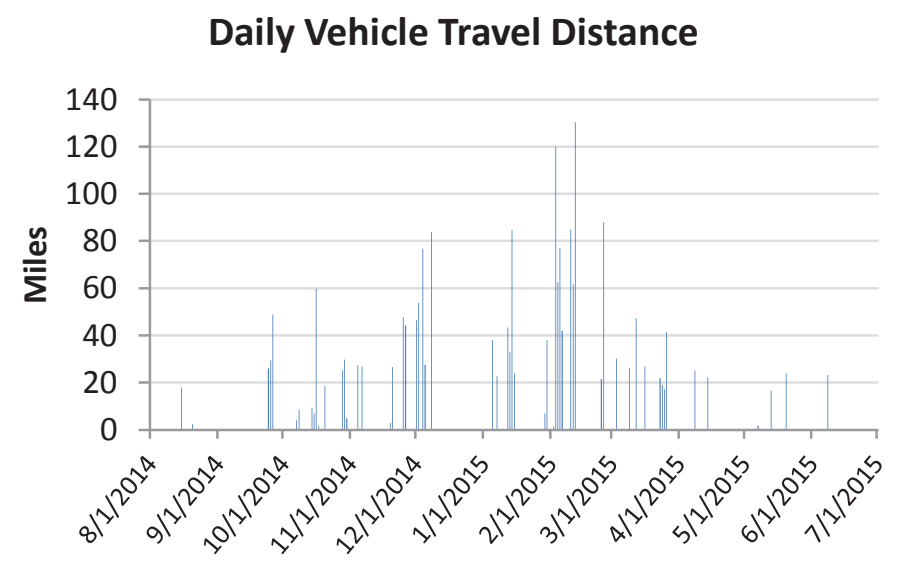

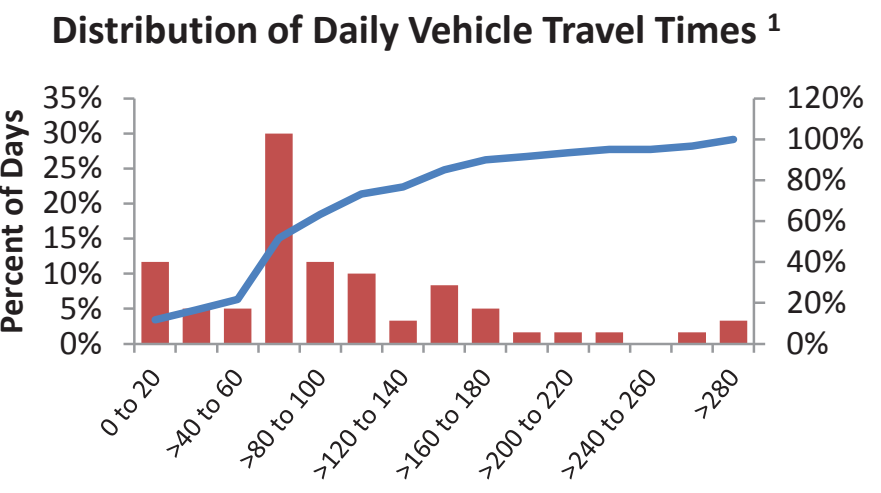

Daily Vehicle Trip Times ( $\mathrm{min})$

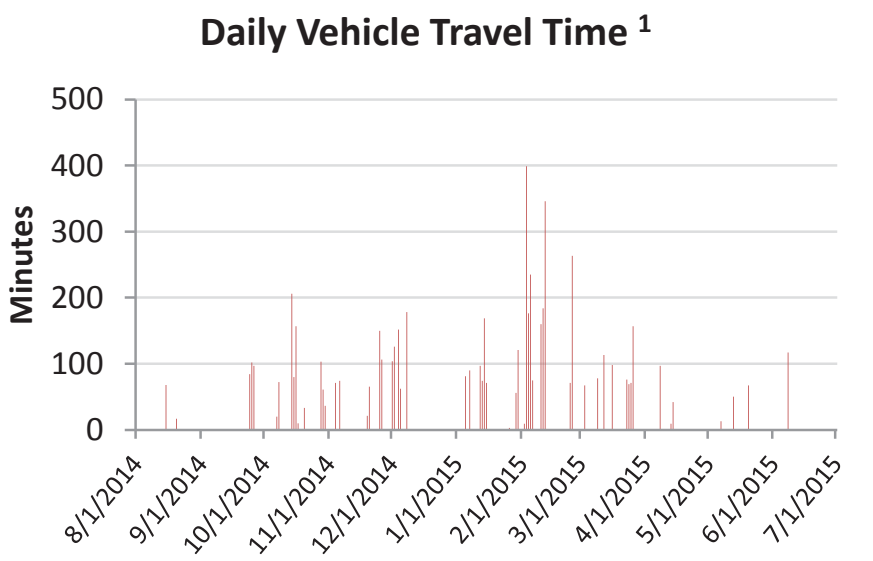

Note 1: Travel time includes all time when vehicle is in the "key on" state, even when the vehicle is not moving

$\begin{array}{ccc}\text { EV Range } & \begin{array}{c}\text { Percentage of } \\ \text { days vehicle } \\ \text { exceeds EV range }\end{array} & \begin{array}{c}\text { Number of days } \\ \text { vehicle exceeds } \\ \text { EV range }\end{array} \\ 69 & 18 \% & 11 \\ 63 & 13 \% & 8 \\ 67 & 13 \% & 8\end{array}$


Vehicle:

Report Period:

Model Year:

Vehicle Make:

Vehicle Model:

Body Type:
5L-G421920L

8/1/2014 00:00:00 - 7/1/2015 00:00:00

2011

FORD

F-150

pickup
Total Number of Days with Driving Average Number of Trips

Average Trip Distance

Total Number of Trips

Total Distance (miles)

Total Trip Duration (minutes)
269

6.9

53.4

1846

14373.6

33650

\section{Distribution of Daily Vehicle Travel Distance}

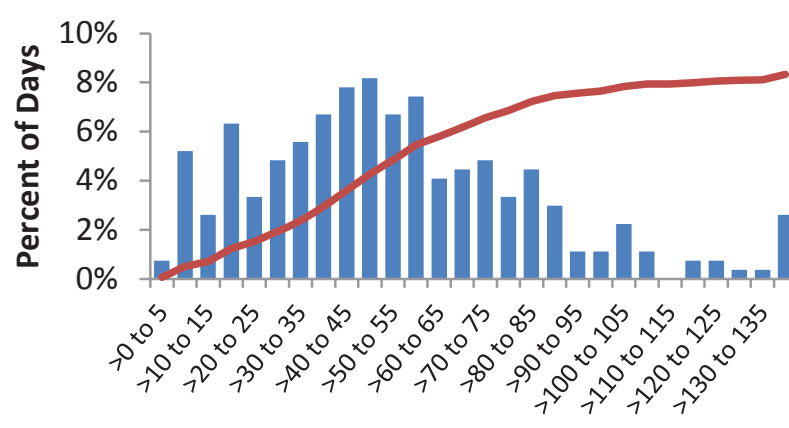

Daily Vehicle Miles Traveled

\section{Daily Vehicle Travel Distance}

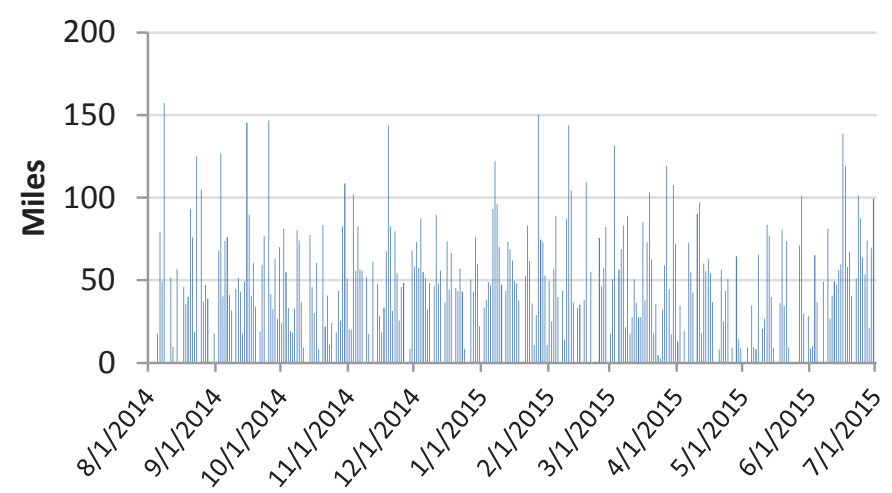

Distribution of Daily Vehicle Travel Times ${ }^{1}$

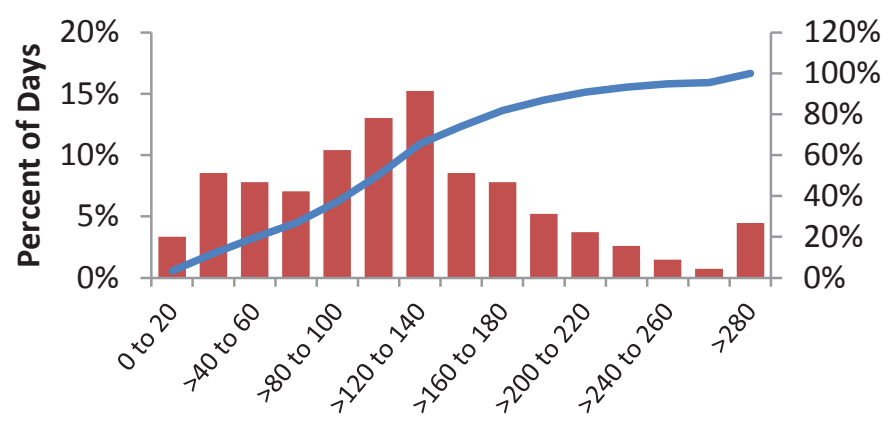

Daily Vehicle Trip Times ( $\mathrm{min})$

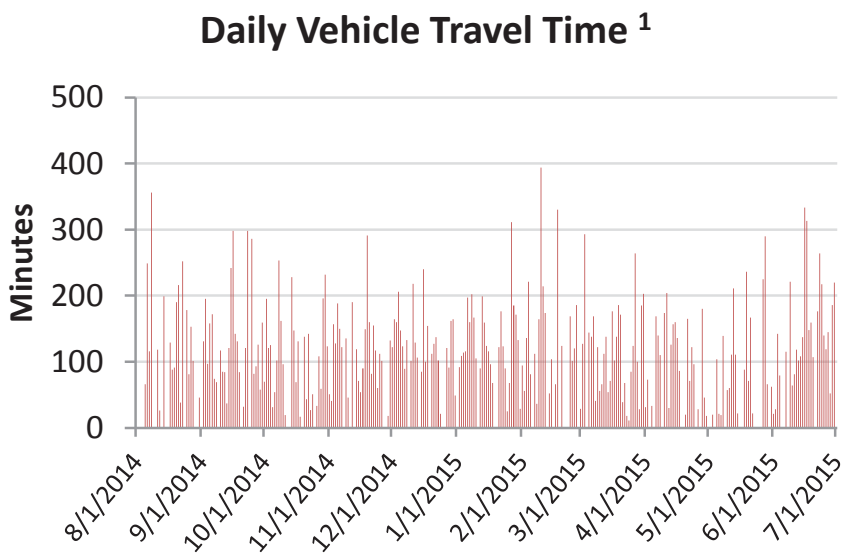

Note 1: Travel time includes all time when vehicle is in the "key on" state, even when the vehicle is not moving

$\begin{array}{cc}\text { Percentage of } & \text { Number of days } \\ \text { days vehicle } & \text { vehicle exceeds }\end{array}$

EV Range

59

63

67

xceeds EV range

$36 \%$

$32 \%$

$29 \%$
EV range

96

85

78
$120 \%$

$80 \%$

$60 \%$

$40 \%$

$20 \%$

$0 \%$ 
Vehicle:

Report Period:

Model Year:

Vehicle Make:

Vehicle Model:

Body Type:
5L-G421775L

8/1/2014 00:00:00 - 7/1/2015 00:00:00

2011

FORD

F-150

pickup

Total Number of Days with Driving

232

12.4

25.4

2886

5882.2

36586

\section{Distribution of Daily Vehicle Travel Times ${ }^{1}$}

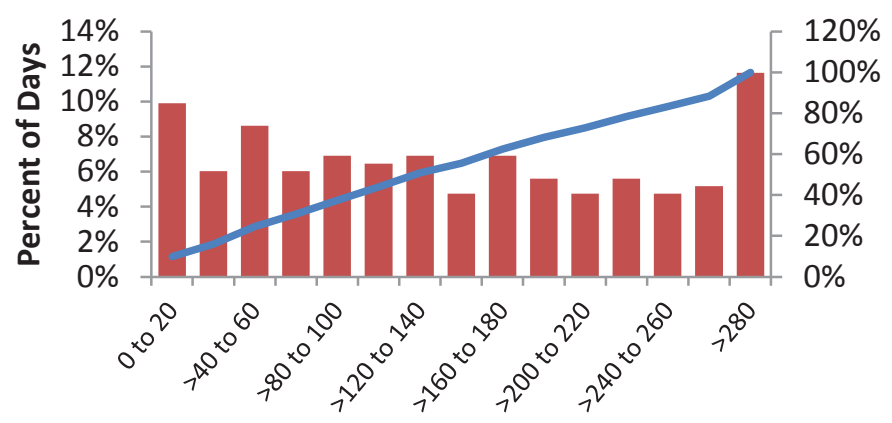

Daily Vehicle Trip Times (min)

Daily Vehicle Miles Traveled
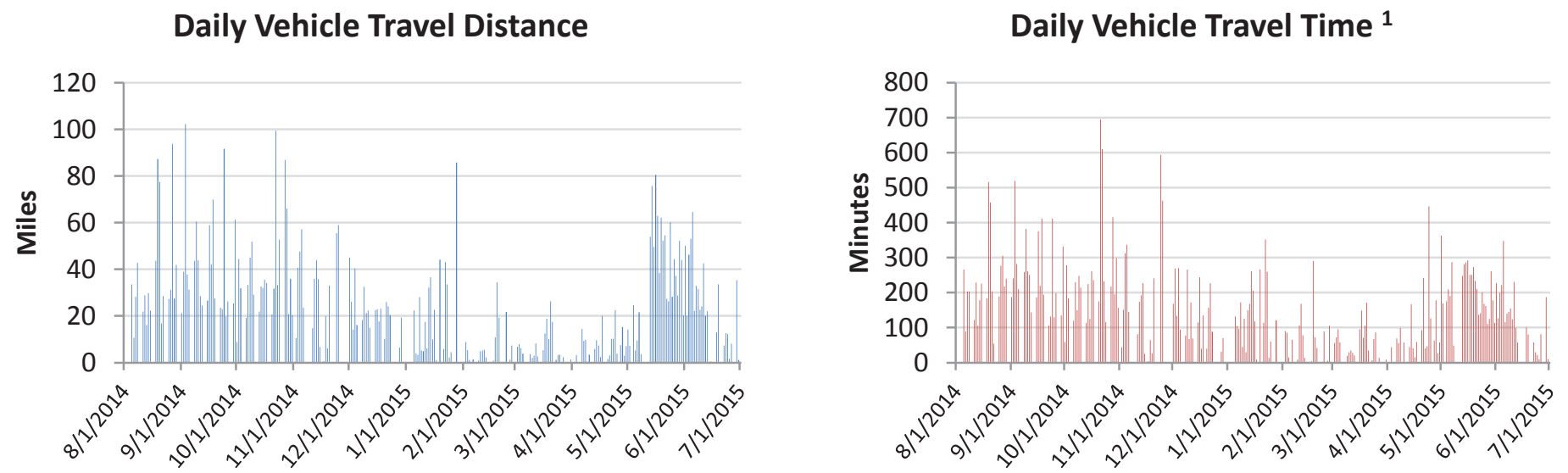

Note 1: Travel time includes all time when vehicle is in the "key on" state, even when the vehicle is not moving

$\begin{array}{cc}\text { Percentage of } & \text { Number of days } \\ \text { days vehicle } & \text { vehicle exceeds }\end{array}$

EV Range

59

63

67 exceeds EV range

$8 \%$

$6 \%$

$5 \%$
EV range

18

13

11 
Vehicle:

Report Period:

Model Year:

Vehicle Make:

Vehicle Model:

Body Type:
5L-G421041D

8/1/2014 00:00:00 - 7/1/2015 00:00:00

2006

Chevrolet

Silverado

pickup

Total Number of Days with Driving

163

4.4

18.5

724

3016.0

9552

Total Trip Duration (minutes)

\section{Distribution of Daily Vehicle Travel Times ${ }^{1}$}

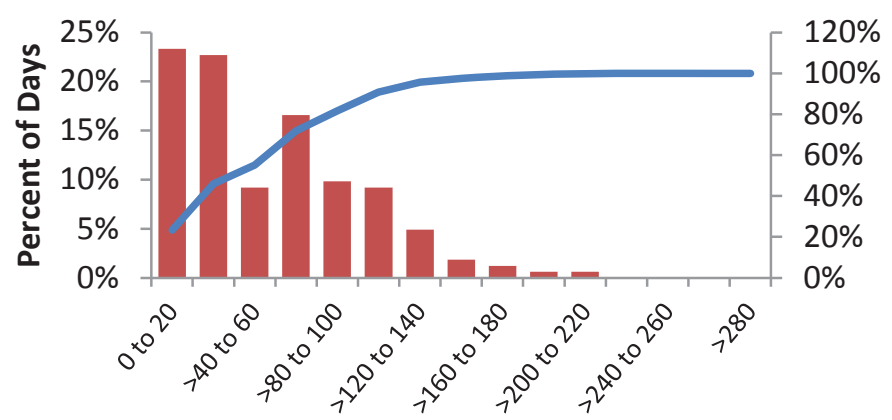

Daily Vehicle Trip Times (min)

Daily Vehicle Miles Traveled
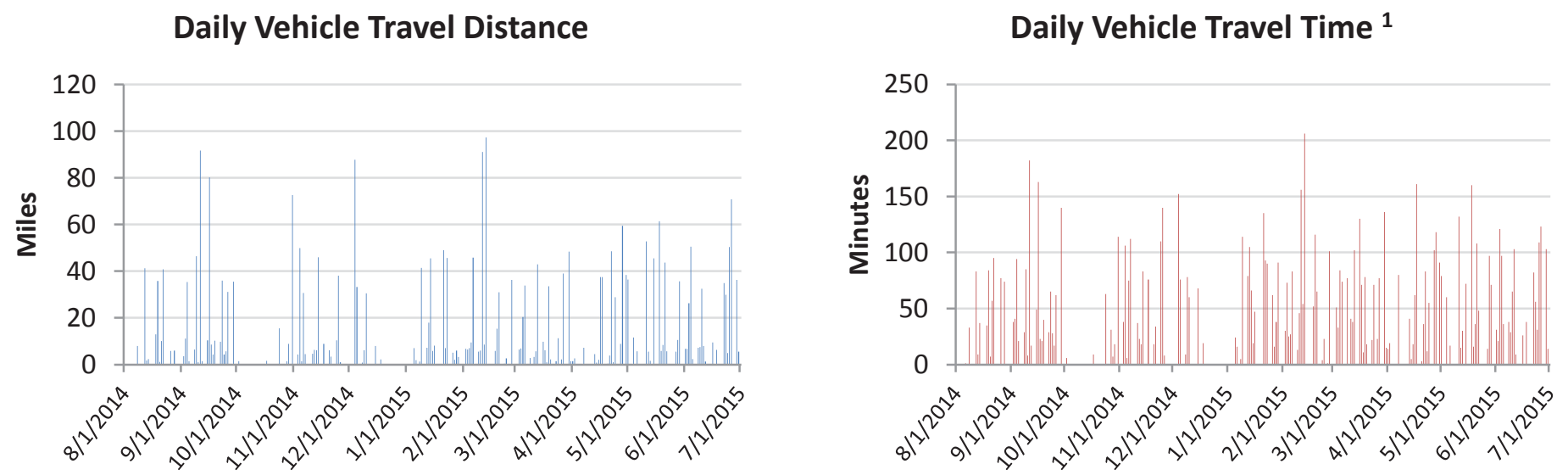

Note 1: Travel time includes all time when vehicle is in the "key on" state, even when the vehicle is not moving

$\begin{array}{cc}\text { Percentage of } & \text { Number of days } \\ \text { days vehicle } & \text { vehicle exceeds }\end{array}$

EV Range

59

63

67 exceeds EV range

$6 \%$

$4 \%$

$4 \%$
EV range

9

7

7 
Vehicle:

Report Period:

Model Year:

Vehicle Make:

Vehicle Model:

Body Type:
5L-G416051H

8/1/2014 00:00:00 - 7/1/2015 00:00:00

2009

Dodge

Grand Caravan

van

Total Number of Days with Driving

Average Number of Trips

0.0

Average Trip Distance

0.0

Total Number of Trips

Total Distance (miles)

0.0

Total Trip Duration (minutes)

\section{Distribution of Daily Vehicle Travel Times ${ }^{1}$}

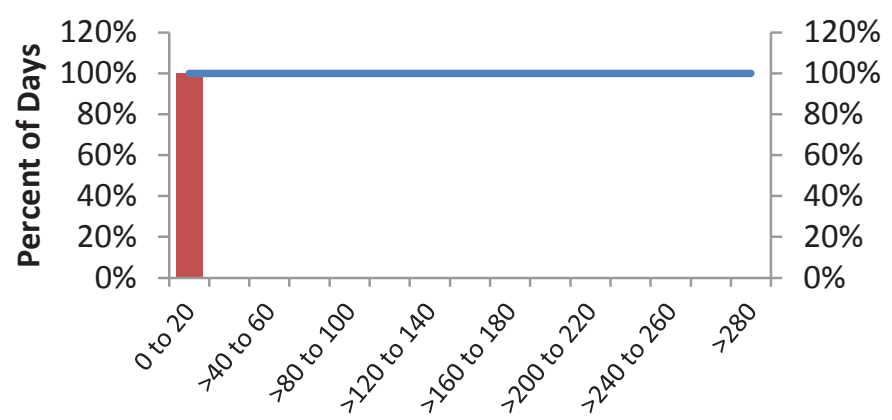

Daily Vehicle Trip Times ( $\min )$

\section{Daily Vehicle Travel Time ${ }^{1}$}

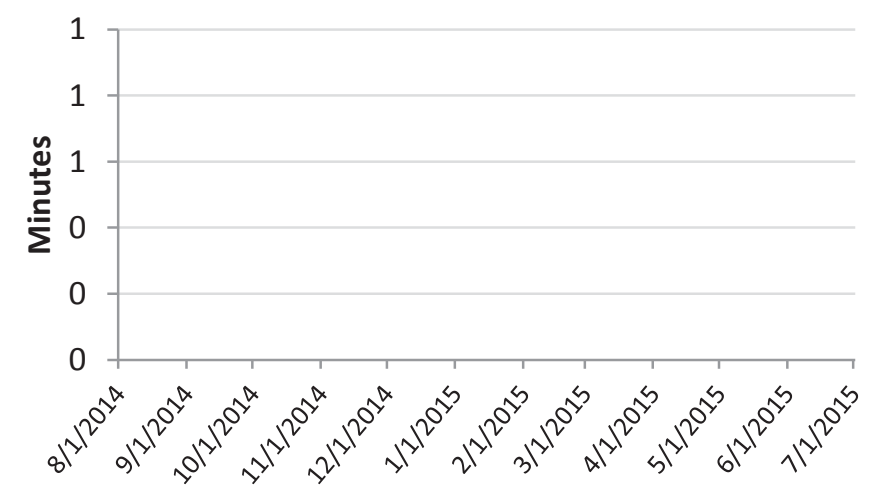

Note 1: Travel time includes all time when vehicle is in the "key on" state, even when the vehicle is not moving

$\begin{array}{cc}\text { Percentage of } & \text { Number of days } \\ \text { days vehicle } & \text { vehicle exceeds }\end{array}$

EV Range

59

63

67 exceeds EV range

$0 \%$

$0 \%$

$0 \%$
EV range

\section{0}

0 
Vehicle:

Report Period:

Model Year:

Vehicle Make:

Vehicle Model:

Body Type:
5L-G414872K

8/1/2014 00:00:00 - 7/1/2015 00:00:00

2010

Dodge

Grand Caravan

van

Total Number of Days with Driving

Average Number of Trips

0.0

Average Trip Distance

0.0

Total Number of Trips

Total Distance (miles)

0.0

Total Trip Duration (minutes)

\section{Distribution of Daily Vehicle Travel Times ${ }^{1}$}

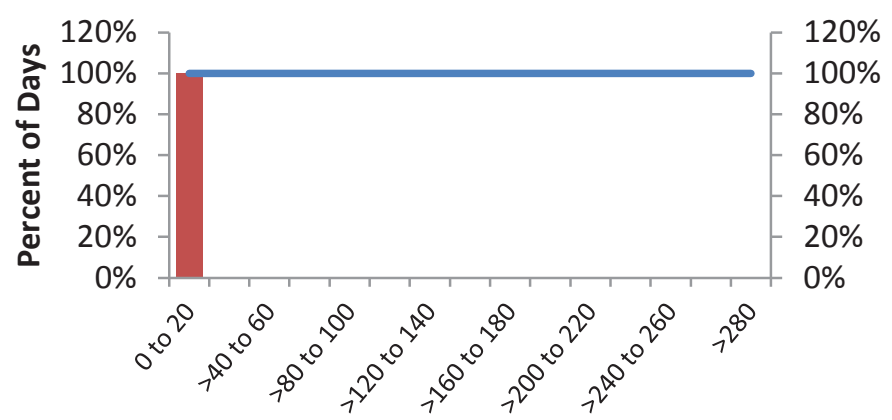

Daily Vehicle Trip Times (min)

\section{Daily Vehicle Travel Time ${ }^{1}$}

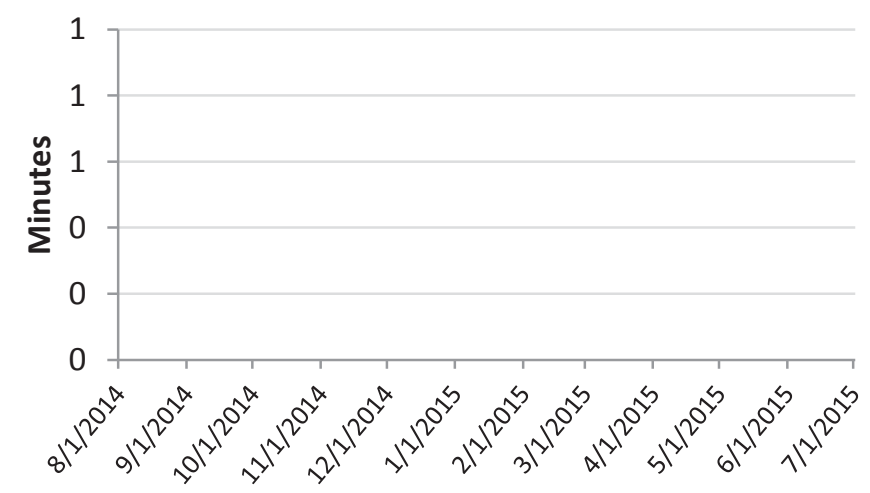

Note 1: Travel time includes all time when vehicle is in the "key on" state, even when the vehicle is not moving

$\begin{array}{cc}\text { Percentage of } & \text { Number of days } \\ \text { days vehicle } & \text { vehicle exceeds }\end{array}$

EV Range

59

63

67 exceeds EV range

$0 \%$

$0 \%$

$0 \%$
EV range

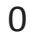


Vehicle:

Report Period:

Model Year:

Vehicle Make:

Vehicle Model:

Body Type:
5L-G414871K

8/1/2014 00:00:00 - 7/1/2015 00:00:00

2010

Dodge

Grand Caravan

van
Total Number of Days with Driving

Average Number of Trips

Average Trip Distance

Total Number of Trips

Total Distance (miles)

Total Trip Duration (minutes)
17

6.2

46.1

105

784.2

1470

\section{Distribution of Daily Vehicle Travel Distance}

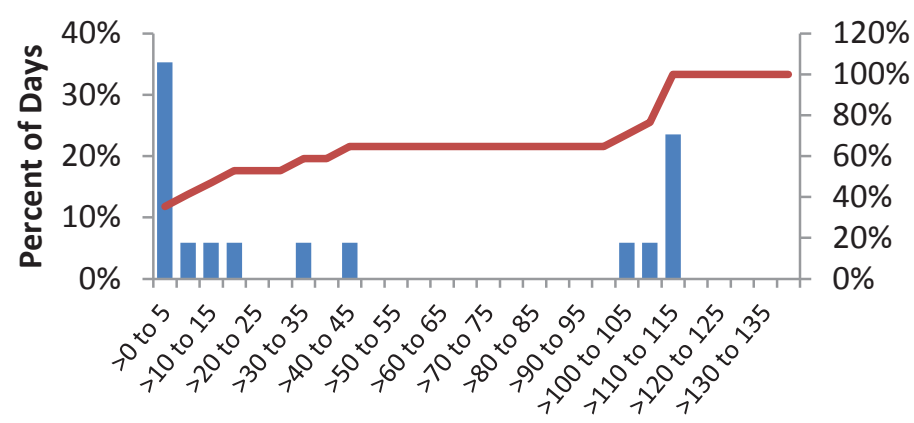

Daily Vehicle Miles Traveled

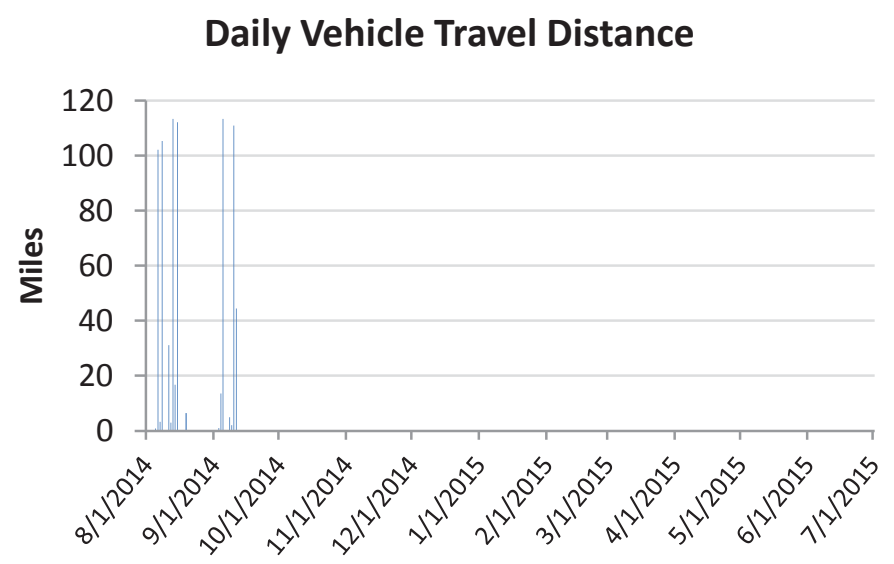

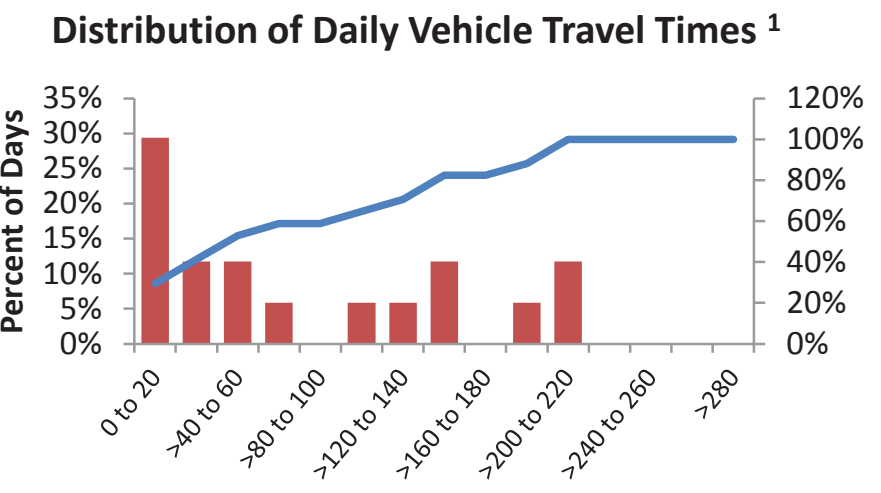

Daily Vehicle Trip Times ( $\mathrm{min})$

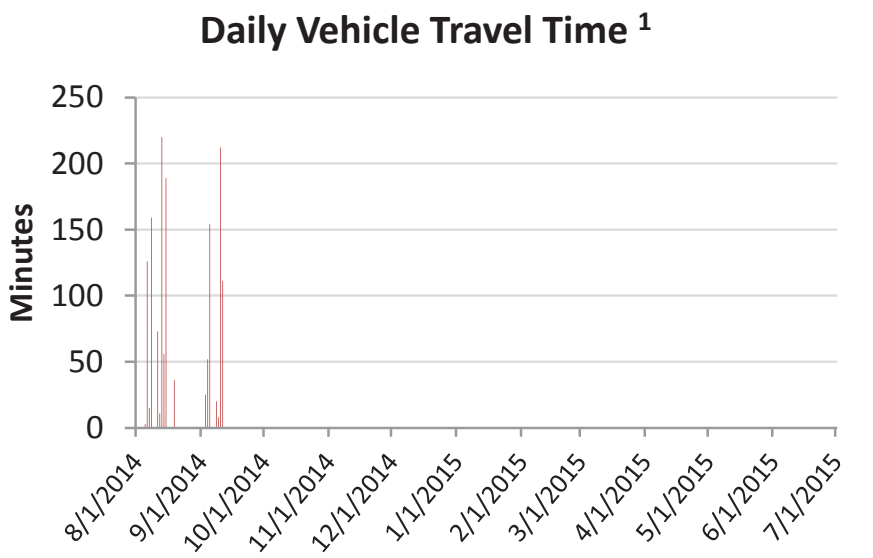

Note 1: Travel time includes all time when vehicle is in the "key on" state, even when the vehicle is not moving

$\begin{array}{cc}\text { Percentage of } & \text { Number of days } \\ \text { days vehicle } & \text { vehicle exceeds }\end{array}$

EV Range

59

63

67 exceeds EV range

$35 \%$

$35 \%$

$35 \%$
EV range

6

6

6 
Vehicle:

Report Period:

Model Year:

Vehicle Make:

Vehicle Model:

Body Type:

Total Number of Days with Driving

Average Number of Trips

Average Trip Distance

Total Number of Trips

Total Distance (miles)

Total Trip Duration (minutes)
5L-G414869K

8/1/2014 00:00:00 - 7/1/2015 00:00:00

2010

FORD

Ranger

small pickup

\section{Distribution of Daily Vehicle Travel Distance}

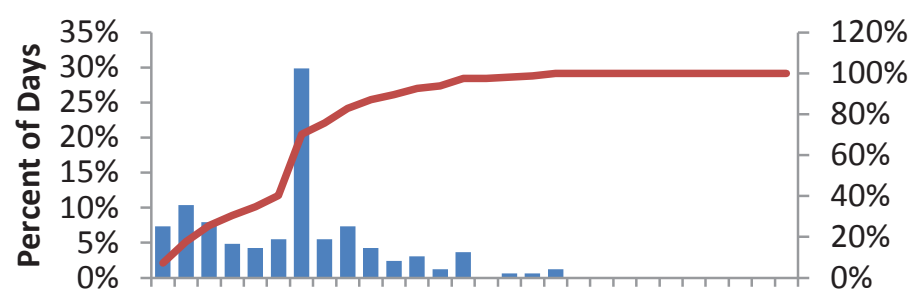

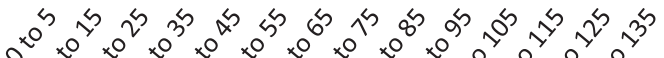

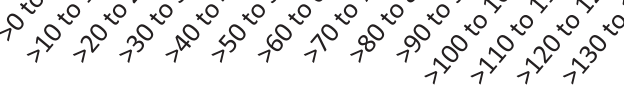

Daily Vehicle Miles Traveled

\section{Daily Vehicle Travel Distance}

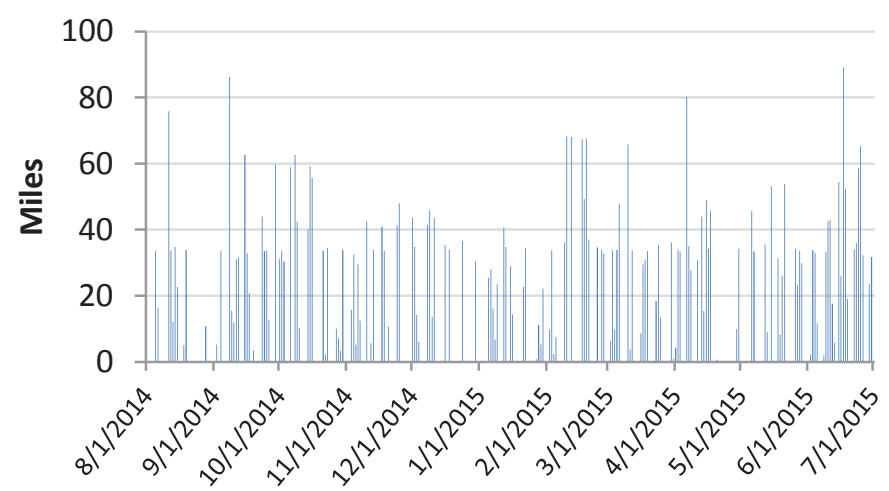

Distribution of Daily Vehicle Travel Times ${ }^{1}$

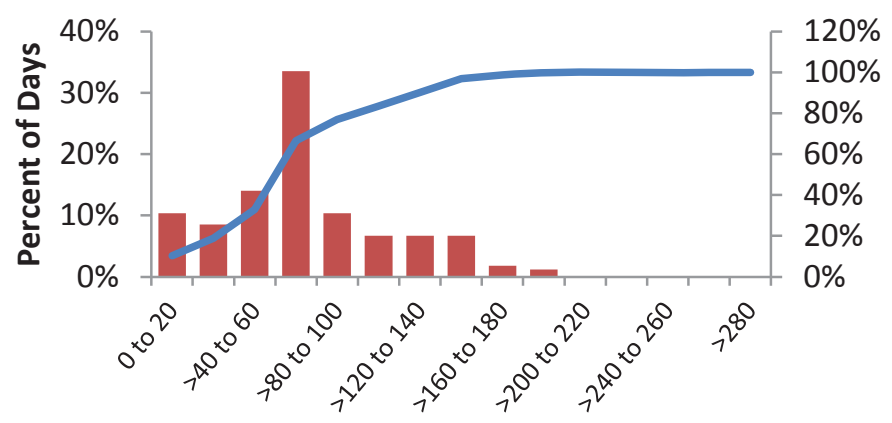

Daily Vehicle Trip Times (min)

\section{Daily Vehicle Travel Time ${ }^{1}$}

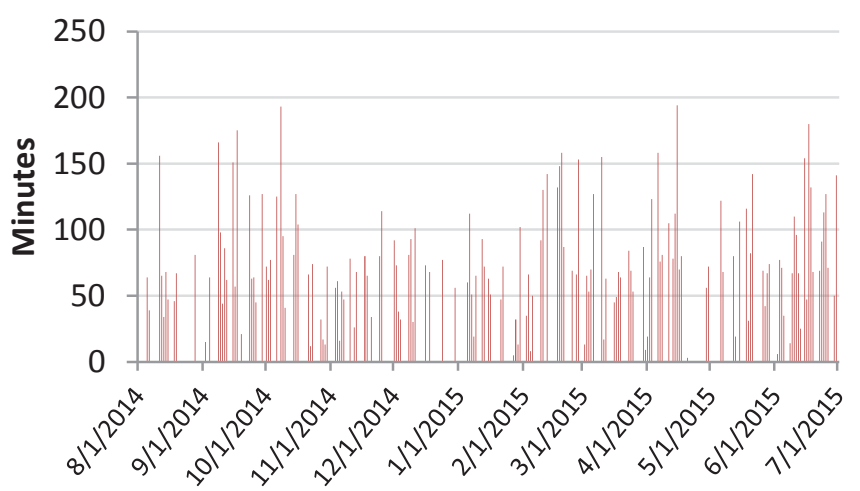

Note 1: Travel time includes all time when vehicle is in the "key on" state, even when the vehicle is not moving

$\begin{array}{cc}\text { Percentage of } & \text { Number of days } \\ \text { days vehicle } & \text { vehicle exceeds }\end{array}$

EV Range

59

63

67 exceeds EV range

$9 \%$

$6 \%$

$5 \%$
EV range

14

10 
Vehicle:

Report Period:

Model Year:

Vehicle Make:

Vehicle Model:

Body Type:

Total Number of Days with Driving

Average Number of Trips

Average Trip Distance

Total Number of Trips

Total Distance (miles)

Total Trip Duration (minutes)
5L-G414868K

8/1/2014 00:00:00 - 7/1/2015 00:00:00

2010

FORD

Ranger

small pickup

\section{Distribution of Daily Vehicle Travel Distance}

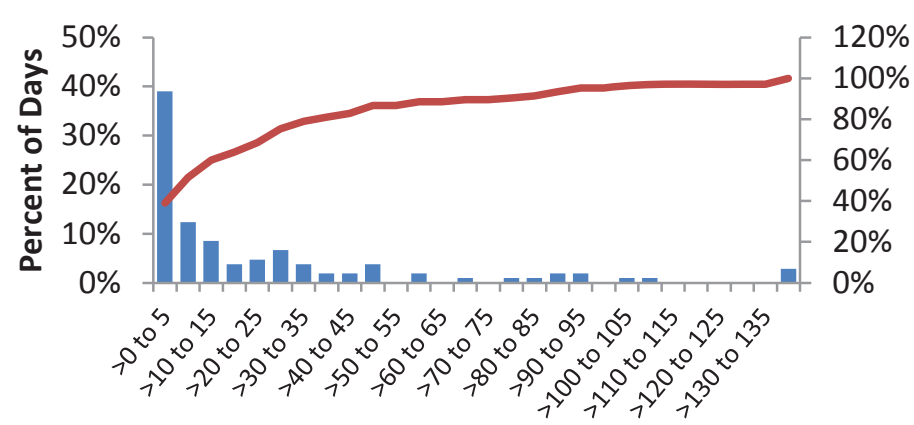

Daily Vehicle Miles Traveled

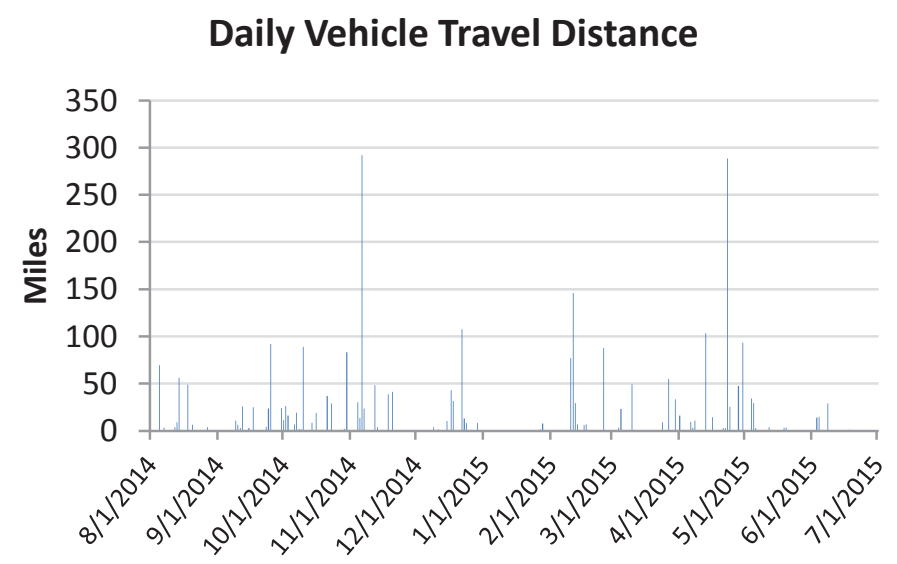

105

5.0

26.6

529

2794.8

6323

\section{Distribution of Daily Vehicle Travel Times ${ }^{1}$}

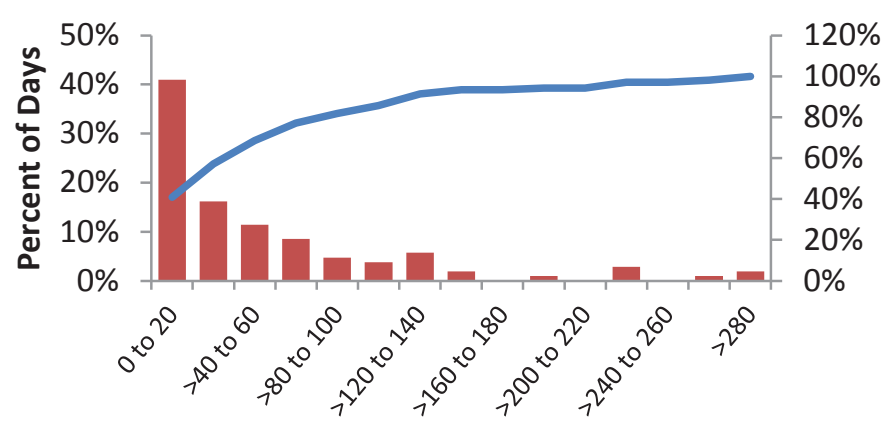

Daily Vehicle Trip Times (min)

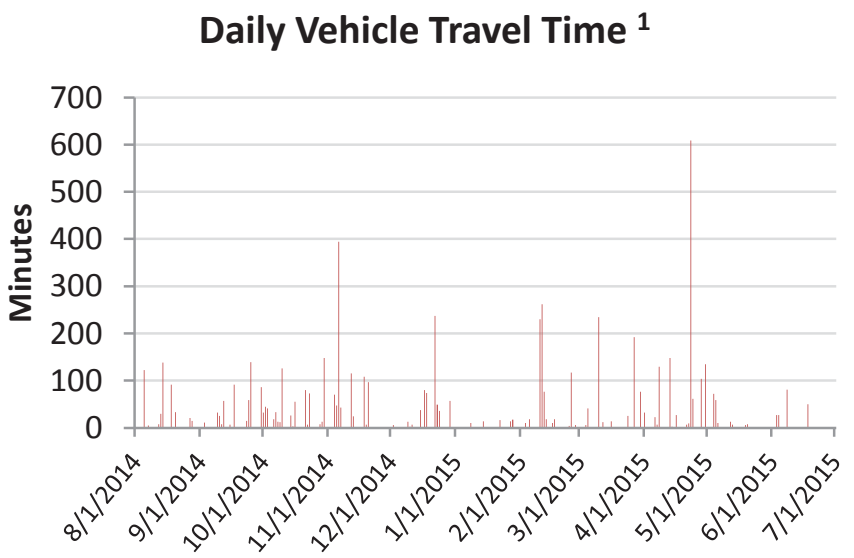

Note 1: Travel time includes all time when vehicle is in the "key on" state, even when the vehicle is not moving

$\begin{array}{cc}\text { Percentage of } & \text { Number of days } \\ \text { days vehicle } & \text { vehicle exceeds }\end{array}$

EV Range

59

63

67 exceeds EV range

$11 \%$

$11 \%$

$11 \%$
EV range

12

12

12 
Vehicle:

Report Period:

Model Year:

Vehicle Make:

Vehicle Model:

Body Type:

Total Number of Days with Driving

Average Number of Trips

Average Trip Distance

Total Number of Trips

Total Distance (miles)

Total Trip Duration (minutes)
5L-G414867K

8/1/2014 00:00:00 - 7/1/2015 00:00:00

2010

FORD

Ranger

small pickup

\section{Distribution of Daily Vehicle Travel Distance}

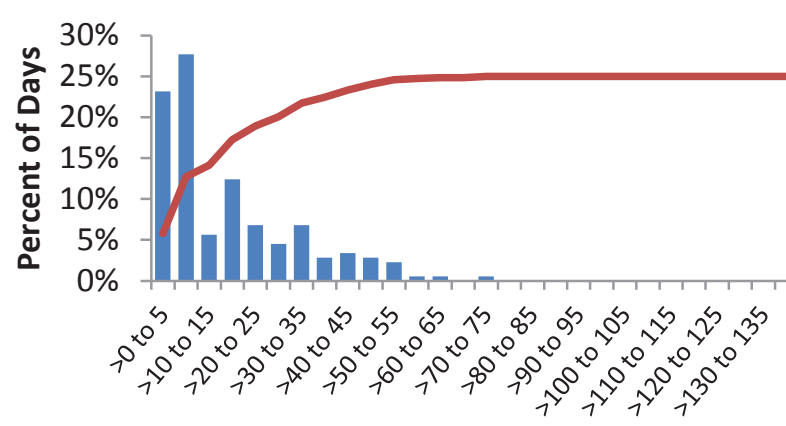

Daily Vehicle Miles Traveled

\section{Daily Vehicle Travel Distance}

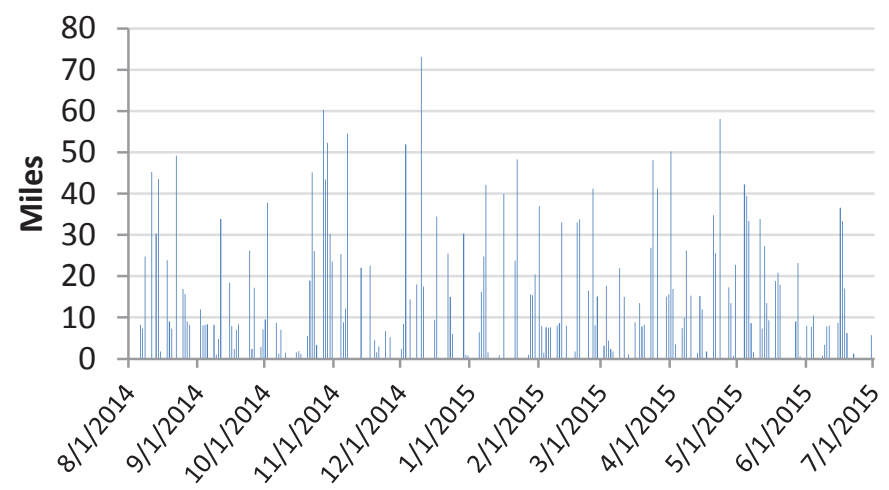

177

4.9

16.5

868

2919.4

6954

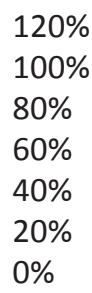

$\%$

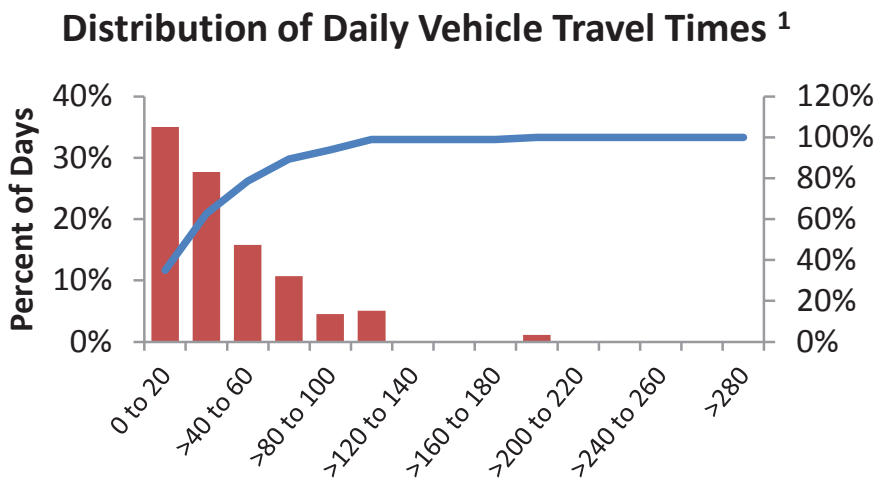

Daily Vehicle Trip Times (min)

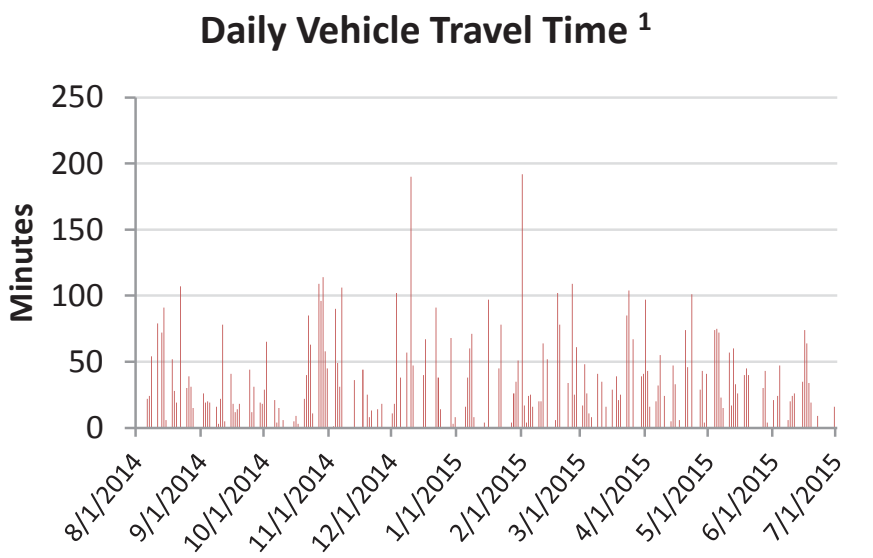

Note 1: Travel time includes all time when vehicle is in the "key on" state, even when the vehicle is not moving

$\begin{array}{ccc}\text { EV Range } & \begin{array}{c}\text { Percentage of } \\ \text { days vehicle } \\ \text { exceeds EV range }\end{array} & \begin{array}{c}\text { Number of days } \\ \text { vehicle exceeds } \\ \text { EV range }\end{array} \\ 69 & 1 \% & 2 \\ 63 & 1 \% & 1 \\ 67 & 1 \% & 1\end{array}$


Vehicle:

Report Period:

Model Year:

Vehicle Make:

Vehicle Model:

Body Type:

Total Number of Days with Driving

Average Number of Trips

Average Trip Distance

Total Number of Trips

Total Distance (miles)

Total Trip Duration (minutes)
5L-G414513L

8/1/2014 00:00:00 - 7/1/2015 00:00:00

2011

RAM

Dakota

small pickup

\section{Distribution of Daily Vehicle Travel Distance}

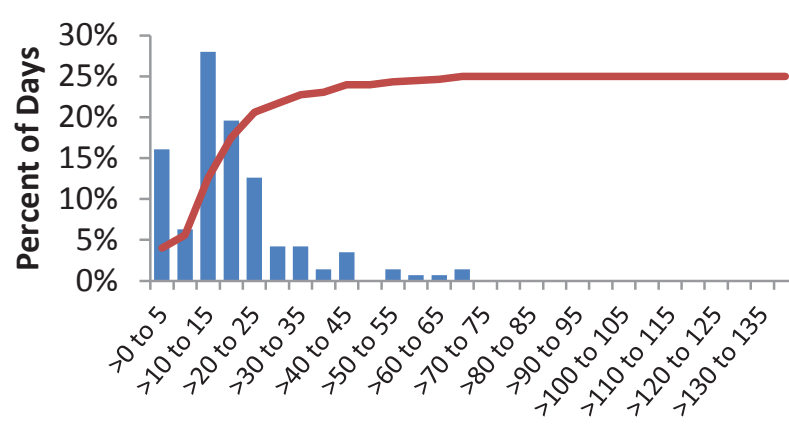

Daily Vehicle Miles Traveled

\section{Daily Vehicle Travel Distance}

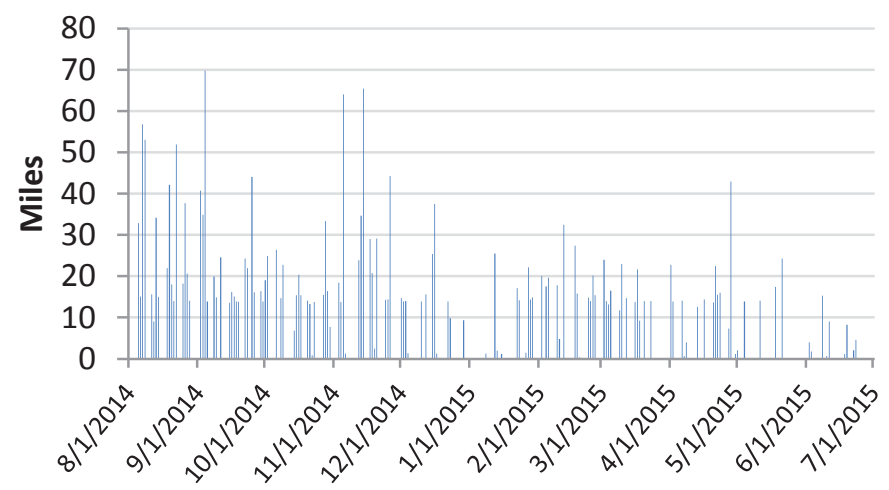

143

3.5

18.0

495

2569.9

5209

Note 1: Travel time includes all time when vehicle is in the "key on" state, even when the vehicle is not moving

$\begin{array}{cc}\text { Percentage of } & \text { Number of days } \\ \text { days vehicle } & \text { vehicle exceeds }\end{array}$

EV Range

59

63

67 exceeds EV range

$2 \%$

$2 \%$

$1 \%$
EV range

3

3

1
$120 \%$
$100 \%$
$80 \%$
$60 \%$
$40 \%$
$20 \%$
$0 \%$

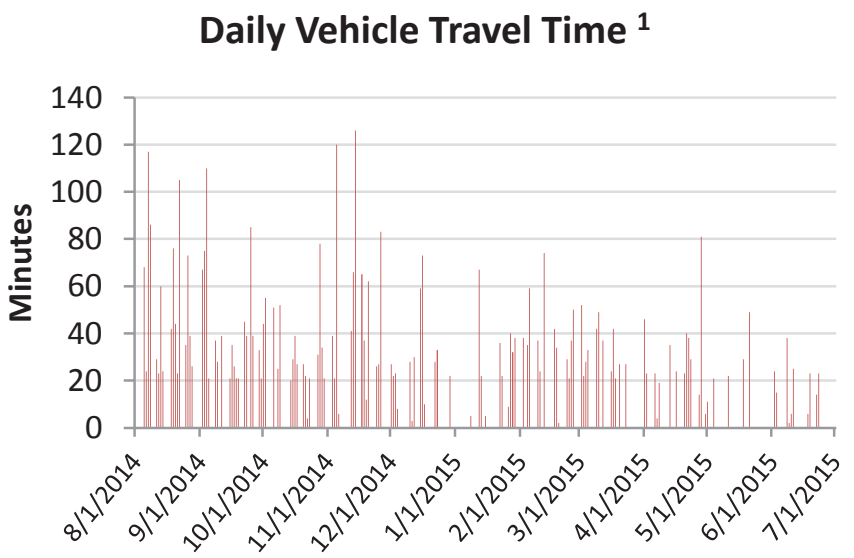

Daily Vehicle Trip Times (min)

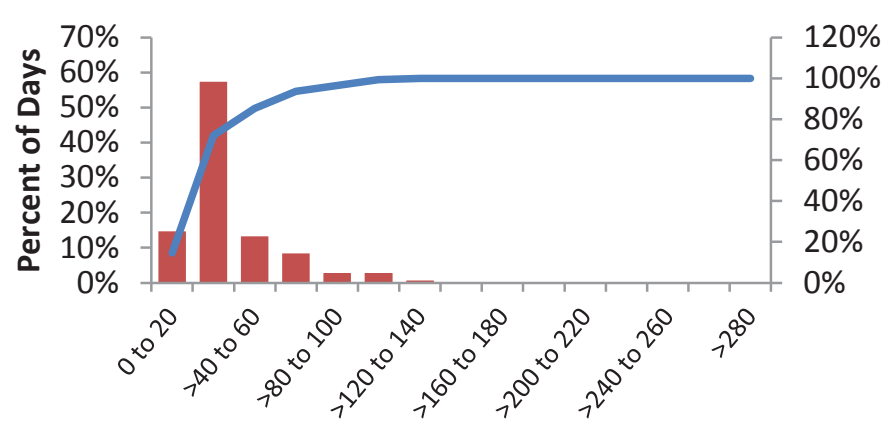


Vehicle:

Report Period:

Model Year:

Vehicle Make:

Vehicle Model:

Body Type:

Total Number of Days with Driving

Average Number of Trips

Average Trip Distance

Total Number of Trips

Total Distance (miles)

Total Trip Duration (minutes)
5L-G414511L

8/1/2014 00:00:00 - 7/1/2015 00:00:00

2011

RAM

Dakota

small pickup

\section{Distribution of Daily Vehicle Travel Distance}

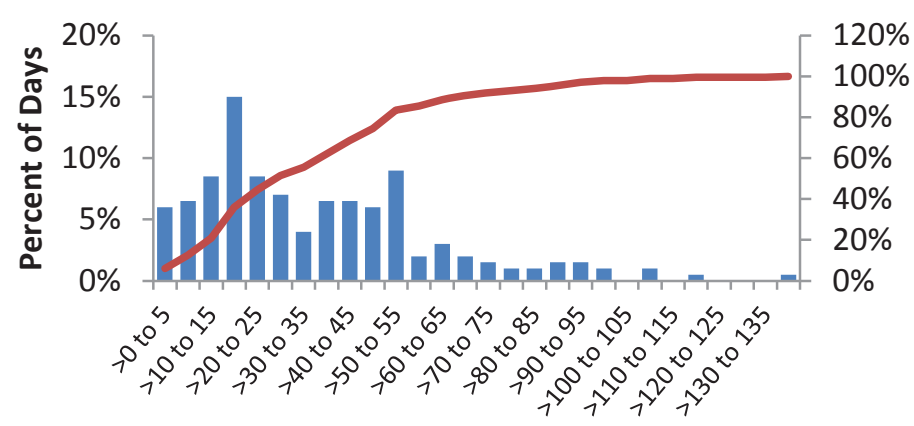

Daily Vehicle Miles Traveled

\section{Daily Vehicle Travel Distance}

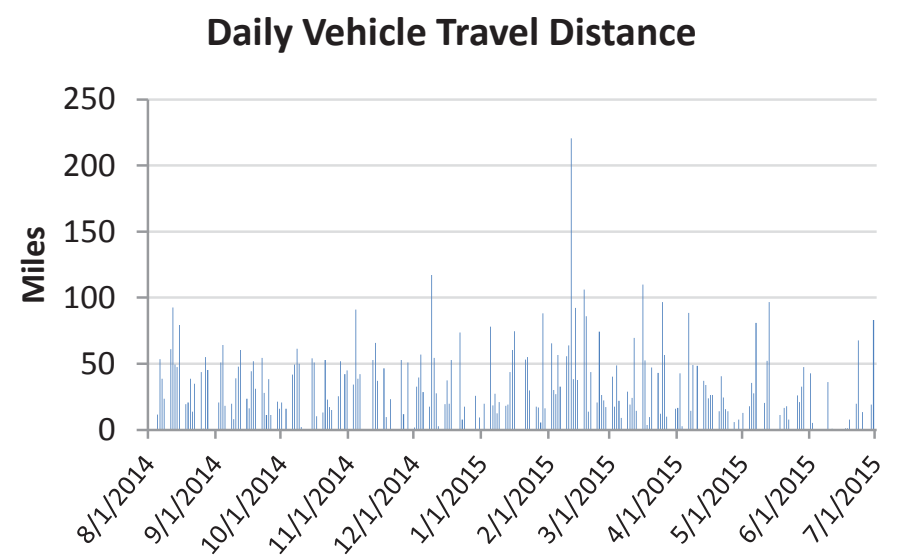

200

6.0

35.8

1199

7157.9

16647
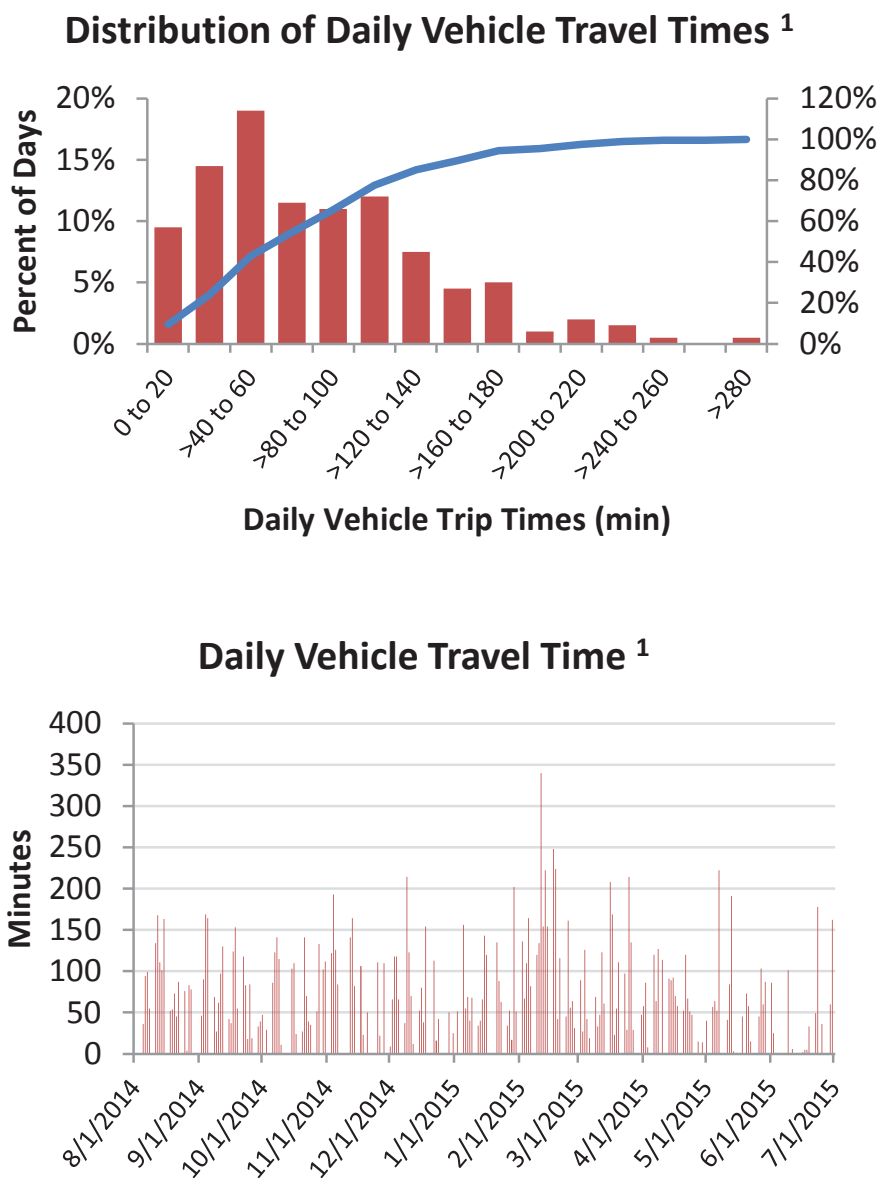

Note 1: Travel time includes all time when vehicle is in the "key on" state, even when the vehicle is not moving

$\begin{array}{ccc}\text { EV Range } & \begin{array}{c}\text { Percentage of } \\ \text { days vehicle } \\ \text { exceeds EV range }\end{array} & \begin{array}{c}\text { Number of days } \\ \text { vehicle exceeds } \\ \text { EV range }\end{array} \\ 59 & 15 \% & 29 \\ 63 & 13 \% & 25 \\ 67 & 11 \% & 21\end{array}$


Vehicle:

Report Period:

Model Year:

Vehicle Make:

Vehicle Model:

Body Type:
5L-G414500L

8/1/2014 00:00:00 - 7/1/2015 00:00:00

2011

Dodge

Grand Caravan

van

Total Number of Days with Driving

Average Number of Trips

Average Trip Distance

Total Number of Trips

Total Distance (miles)

2659.3

Total Trip Duration (minutes)

6434

\section{Distribution of Daily Vehicle Travel Distance}

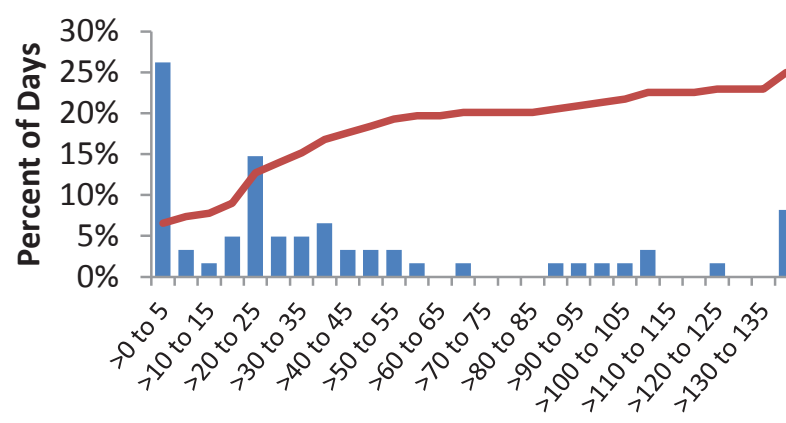

Daily Vehicle Miles Traveled

\section{Daily Vehicle Travel Distance}

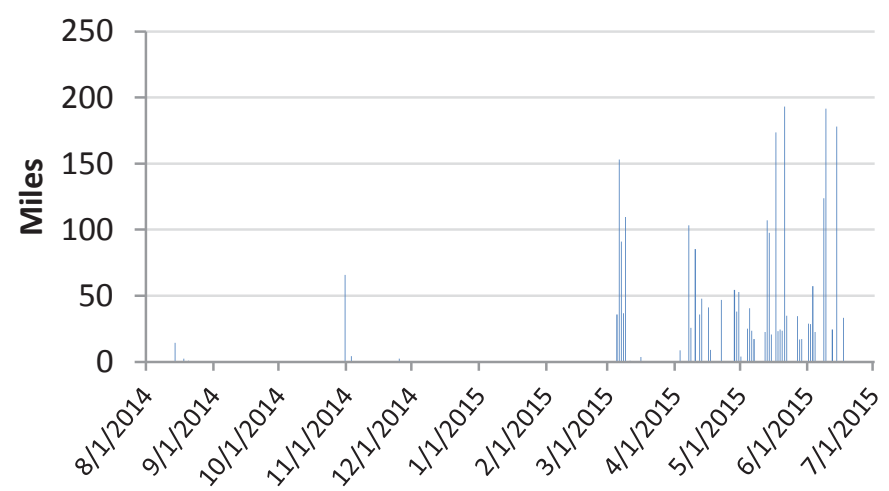

Distribution of Daily Vehicle Travel Times ${ }^{1}$

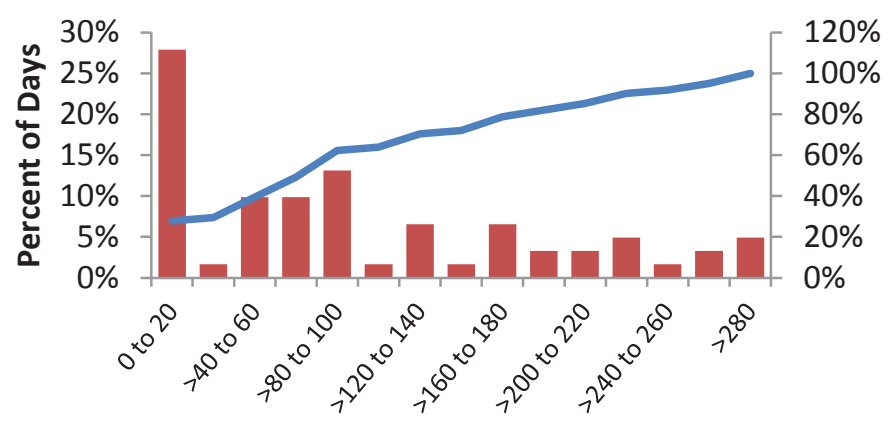

Daily Vehicle Trip Times (min)

\section{Daily Vehicle Travel Time ${ }^{1}$}

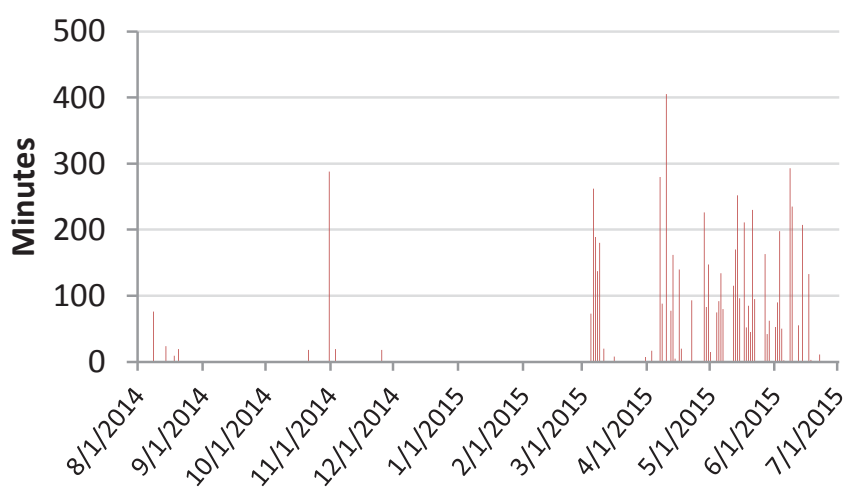

Note 1: Travel time includes all time when vehicle is in the "key on" state, even when the vehicle is not moving

$\begin{array}{cc}\text { Percentage of } & \text { Number of days } \\ \text { days vehicle } & \text { vehicle exceeds }\end{array}$

EV Range

59

63

67 exceeds EV range

$21 \%$

$21 \%$

$20 \%$
EV range

13

13

12 
Vehicle:

Report Period:

Model Year:

Vehicle Make:

Vehicle Model:

Body Type:
5L-G414488L

8/1/2014 00:00:00 - 7/1/2015 00:00:00

2011

Dodge

Grand Caravan

van
Total Number of Days with Driving Average Number of Trips

Average Trip Distance

Total Number of Trips

Total Distance (miles)

Total Trip Duration (minutes)
201

6.0

27.4

1199

5517.0

13552

\section{Distribution of Daily Vehicle Travel Distance}

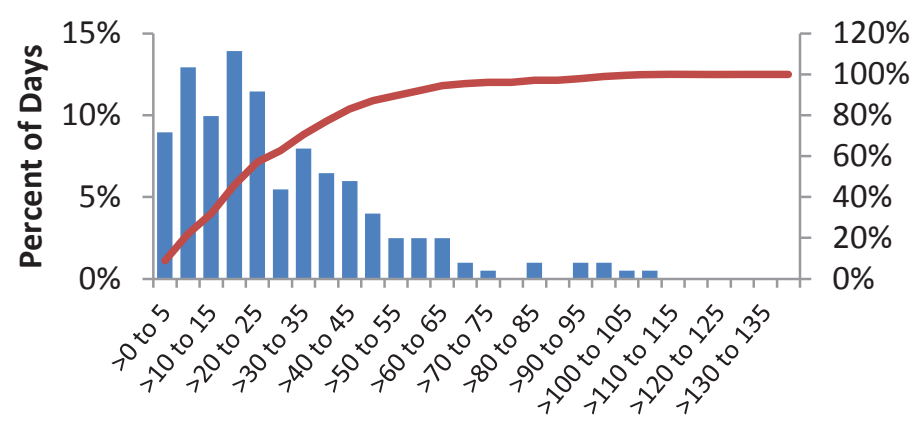

Daily Vehicle Miles Traveled

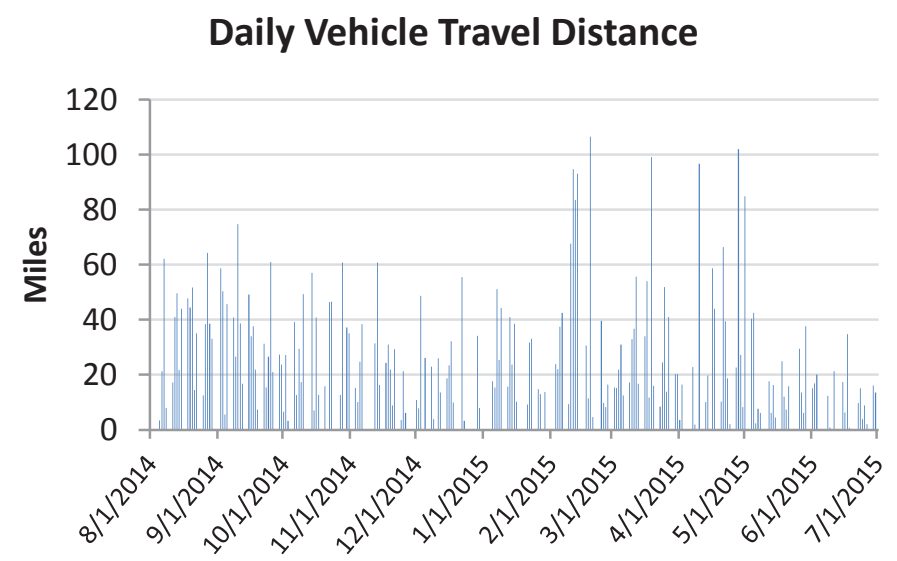

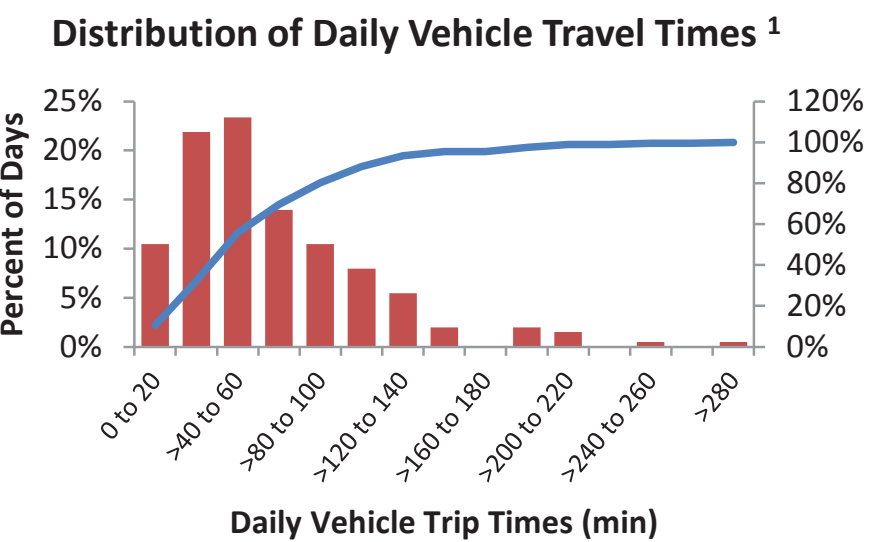

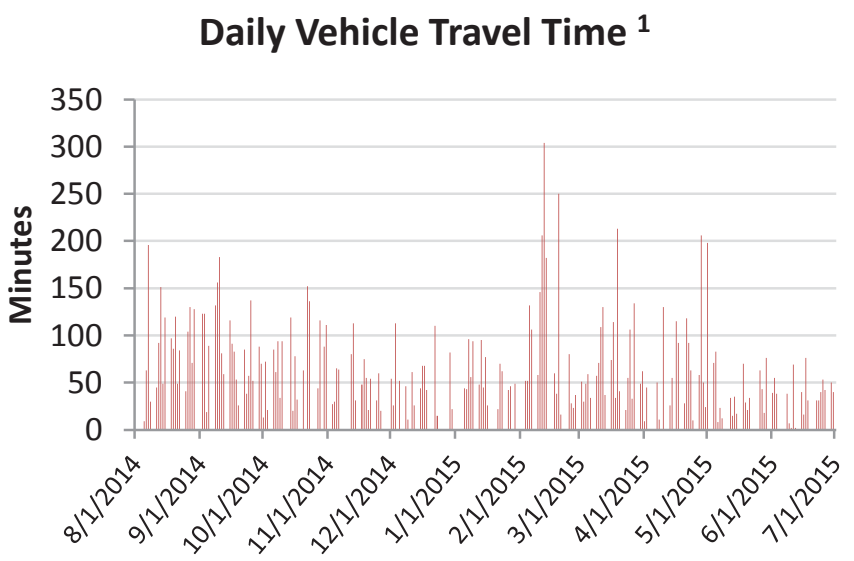

Note 1: Travel time includes all time when vehicle is in the "key on" state, even when the vehicle is not moving

$\begin{array}{ccc}\text { EV Range } & \begin{array}{c}\text { Percentage of } \\ \text { days vehicle } \\ \text { exceeds EV range }\end{array} & \begin{array}{c}\text { Number of days } \\ \text { vehicle exceeds } \\ \text { EV range }\end{array} \\ 59 & 8 \% & 16 \\ 63 & 6 \% & 12 \\ 67 & 5 \% & 10\end{array}$


Vehicle:

Report Period:

Model Year:

Vehicle Make:

Vehicle Model:

Body Type:

Total Number of Days with Driving

Average Number of Trips

Average Trip Distance

Total Number of Trips

Total Distance (miles)

Total Trip Duration (minutes)
5L-G414440H

8/1/2014 00:00:00 - 7/1/2015 00:00:00

2009

Dodge

Dakota

small pickup

\section{Distribution of Daily Vehicle Travel Distance}

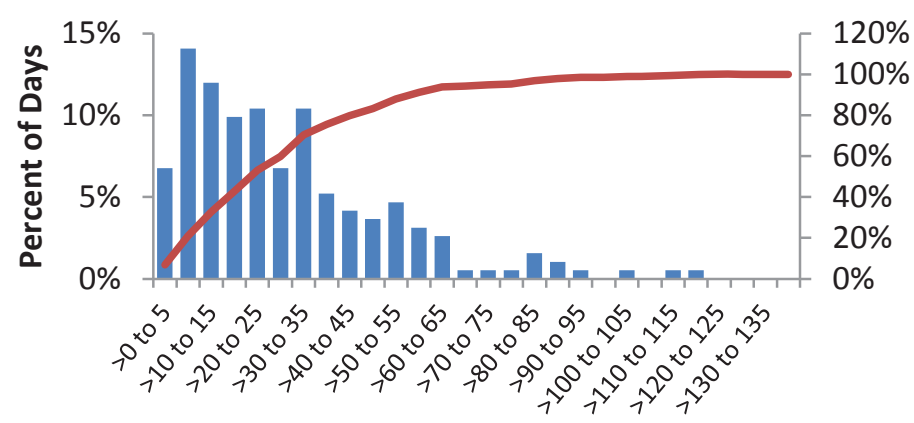

Daily Vehicle Miles Traveled

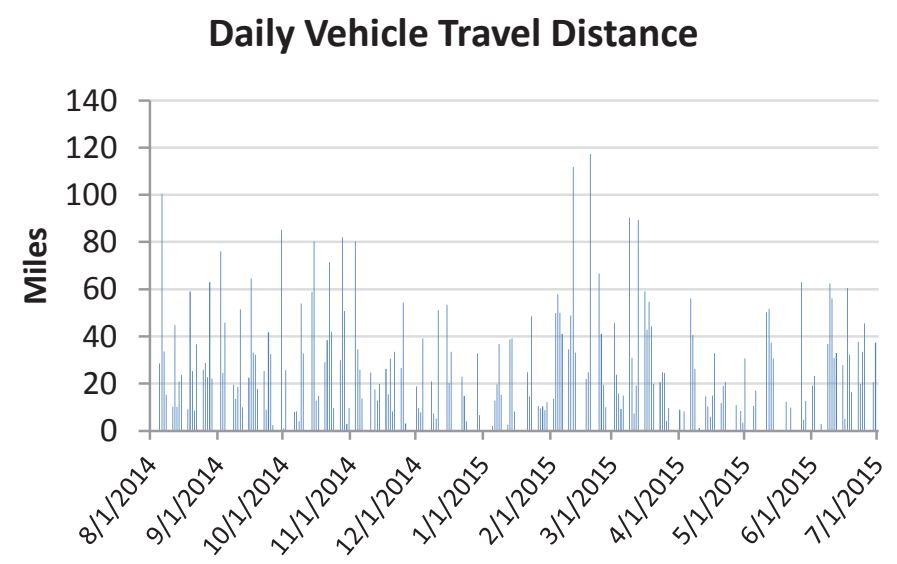

192

5.7

29.1

1097

5589.5

12708
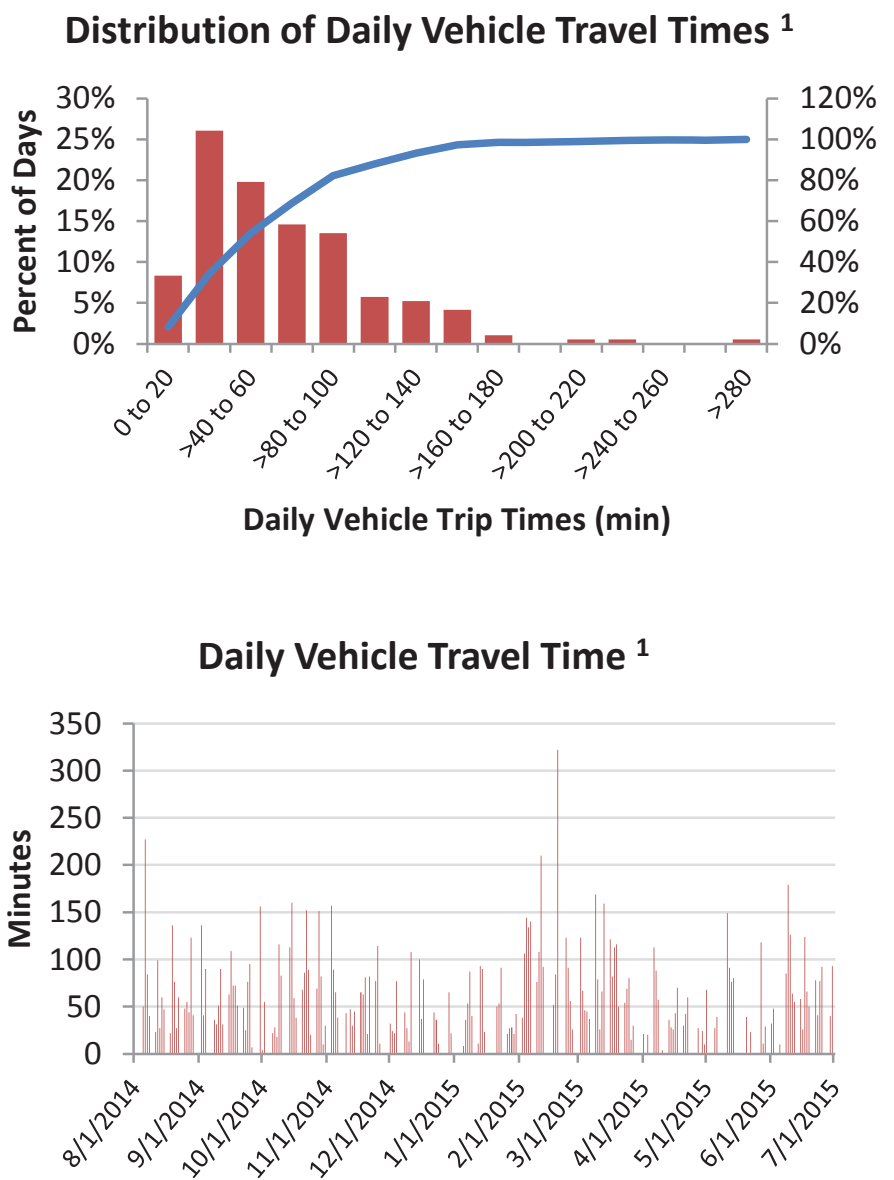

Note 1: Travel time includes all time when vehicle is in the "key on" state, even when the vehicle is not moving

$\begin{array}{ccc}\text { EV Range } & \begin{array}{c}\text { Percentage of } \\ \text { days vehicle } \\ \text { exceeds EV range }\end{array} & \begin{array}{c}\text { Number of days } \\ \text { vehicle exceeds } \\ \text { EV range }\end{array} \\ 59 & 9 \% & 18 \\ 63 & 7 \% & 13 \\ 67 & 6 \% & 11\end{array}$


Vehicle:

Report Period:

Model Year:

Vehicle Make:

Vehicle Model:

Body Type:

Total Number of Days with Driving

Average Number of Trips

Average Trip Distance

Total Number of Trips

Total Distance (miles)

Total Trip Duration (minutes)
5L-G414438H

8/1/2014 00:00:00 - 7/1/2015 00:00:00

2009

Dodge

Dakota

small pickup

\section{Distribution of Daily Vehicle Travel Distance}

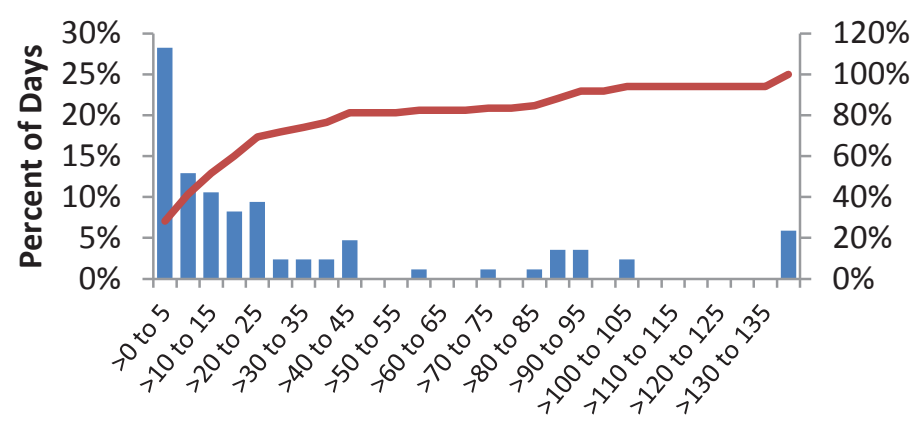

Daily Vehicle Miles Traveled

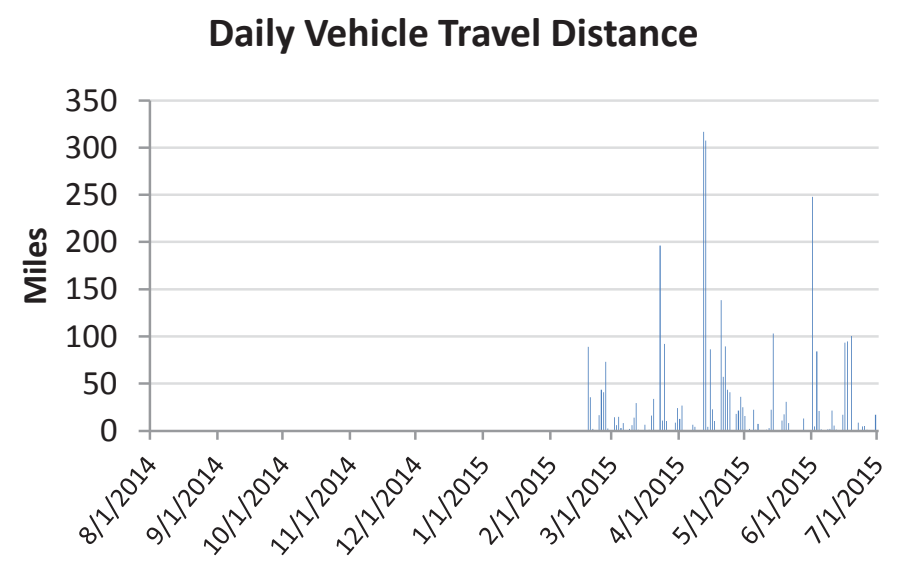

85

6.6

36.1

565

3065.5

7981

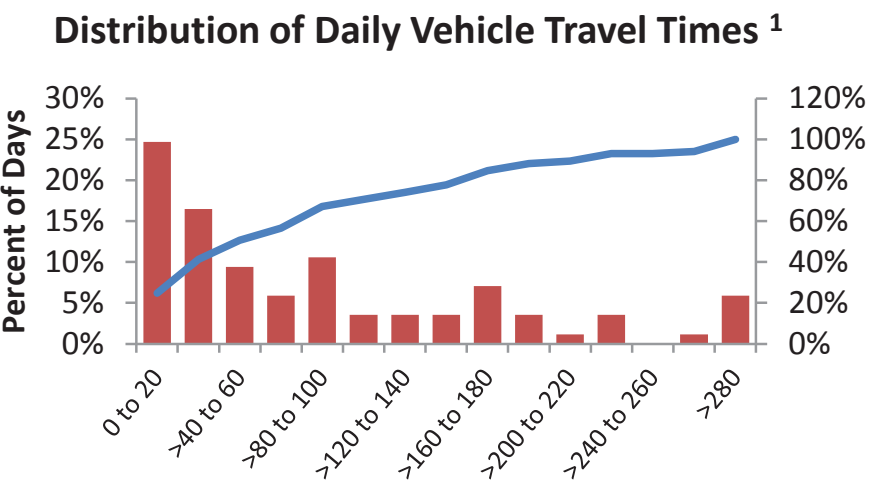

Daily Vehicle Trip Times (min)

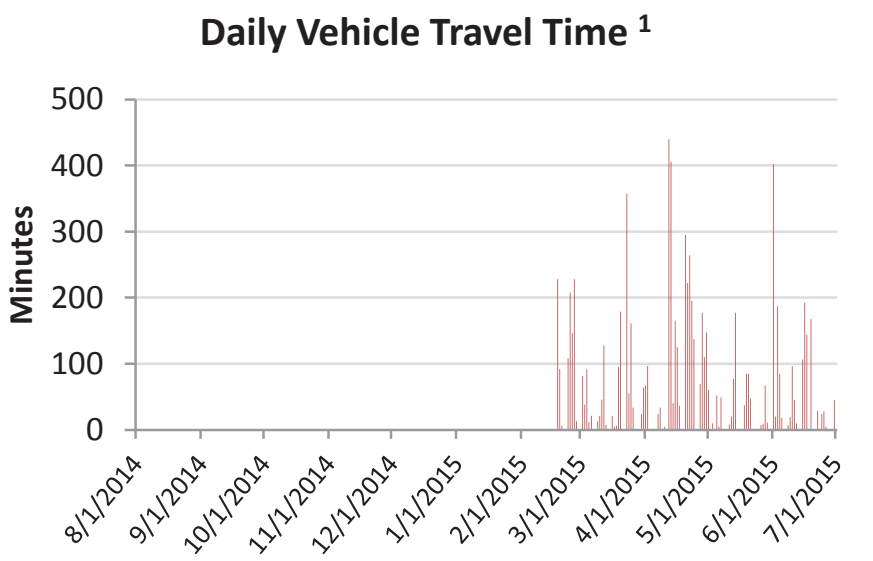

Note 1: Travel time includes all time when vehicle is in the "key on" state, even when the vehicle is not moving

$\begin{array}{ccc}\text { EV Range } & \begin{array}{c}\text { Percentage of } \\ \text { days vehicle } \\ \text { exceeds EV range }\end{array} & \begin{array}{c}\text { Number of days } \\ \text { vehicle exceeds } \\ \text { EV range }\end{array} \\ 69 & 18 \% & 15 \\ 63 & 18 \% & 15 \\ 67 & 18 \% & 15\end{array}$


Vehicle:

Report Period:

Model Year:

Vehicle Make:

Vehicle Model:

Body Type:

Total Number of Days with Driving

Average Number of Trips

Average Trip Distance

Total Number of Trips

Total Distance (miles)

Total Trip Duration (minutes)
5L-G414430H

8/1/2014 00:00:00 - 7/1/2015 00:00:00

2009

Dodge

Dakota SXT

small pickup

\section{Distribution of Daily Vehicle Travel Distance}

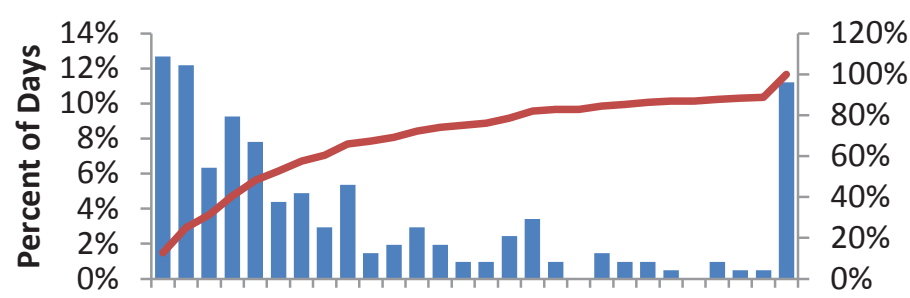

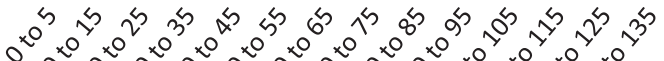

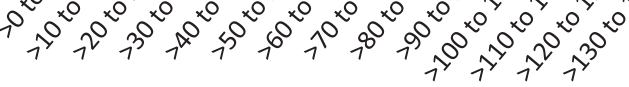

Daily Vehicle Miles Traveled

\section{Daily Vehicle Travel Distance}

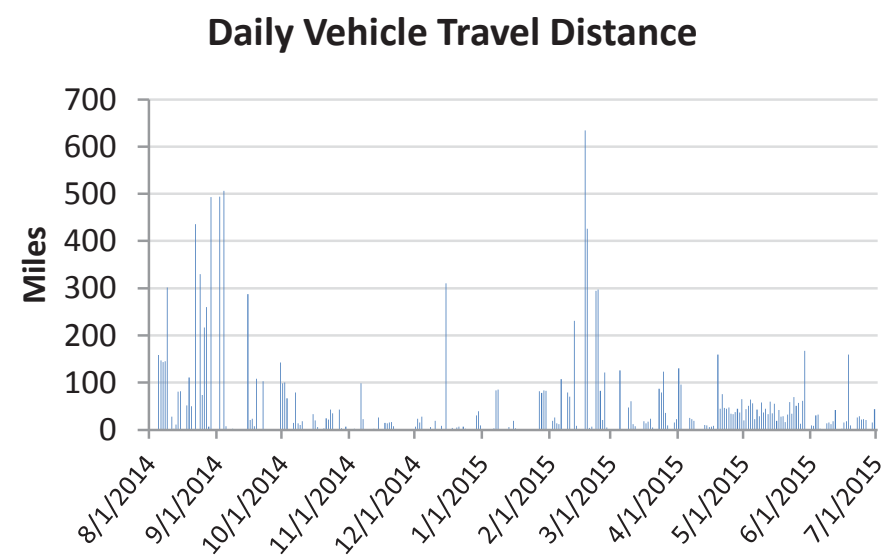

205

8.3

62.5

1709

12810.2

37508

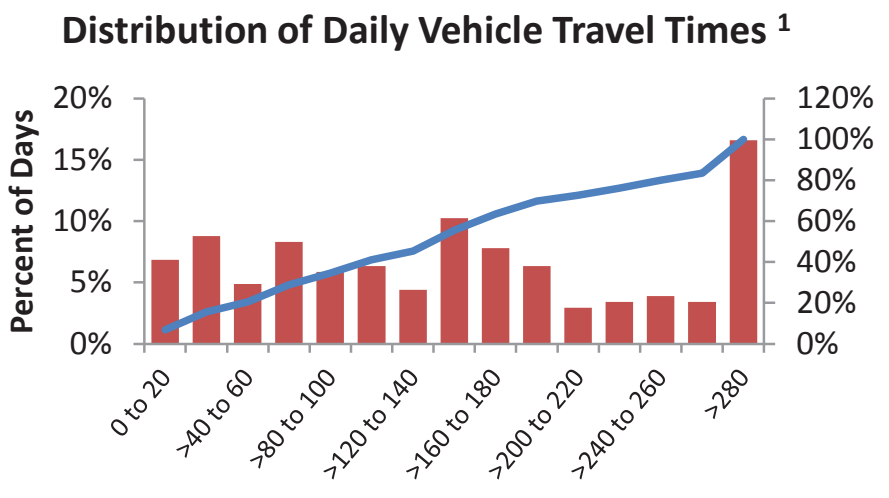

Daily Vehicle Trip Times (min)

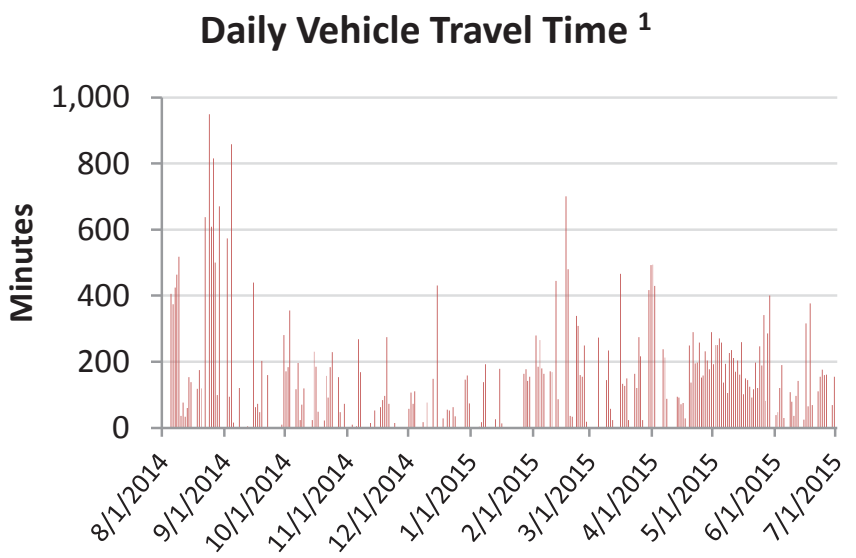

Note 1: Travel time includes all time when vehicle is in the "key on" state, even when the vehicle is not moving

$\begin{array}{cc}\text { Percentage of } & \text { Number of days } \\ \text { days vehicle } & \text { vehicle exceeds }\end{array}$

EV Range

59

63

67 exceeds EV range

$28 \%$

$27 \%$

$25 \%$
EV range

58

55

52 
Vehicle:

Report Period:

Model Year:

Vehicle Make:

Vehicle Model:

Body Type:

Total Number of Days with Driving

Average Number of Trips

Average Trip Distance

Total Number of Trips

Total Distance (miles)

Total Trip Duration (minutes)
5L-G414428H

8/1/2014 00:00:00 - 7/1/2015 00:00:00

2009

Dodge

Dakota SXT

small pickup

\section{Distribution of Daily Vehicle Travel Distance}

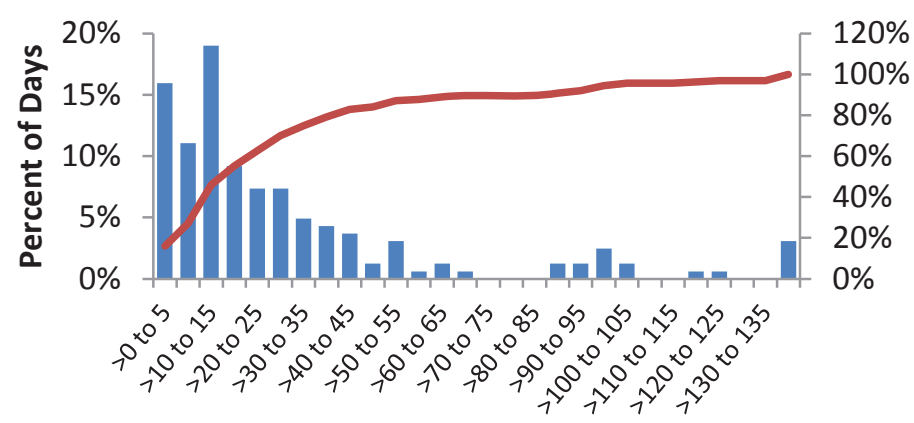

Daily Vehicle Miles Traveled

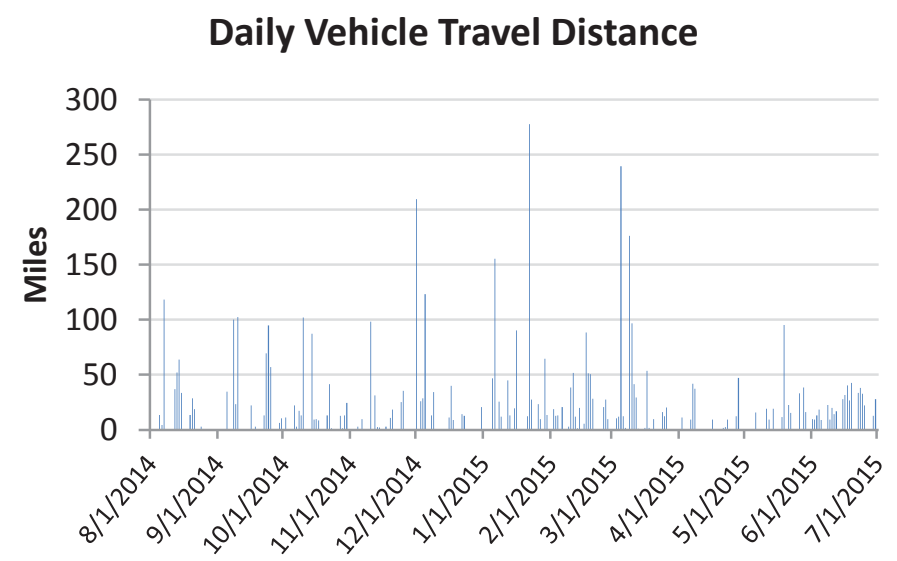

163

5.0

31.3

808

5096.0

11093

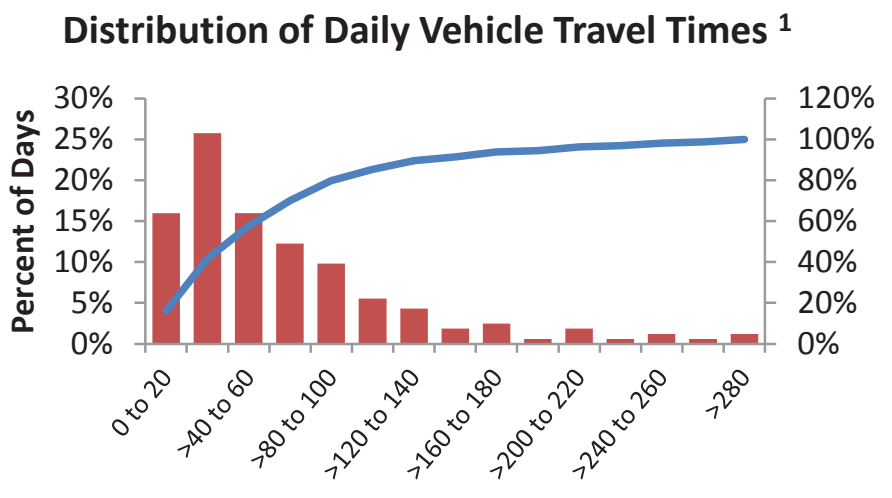

Daily Vehicle Trip Times (min)

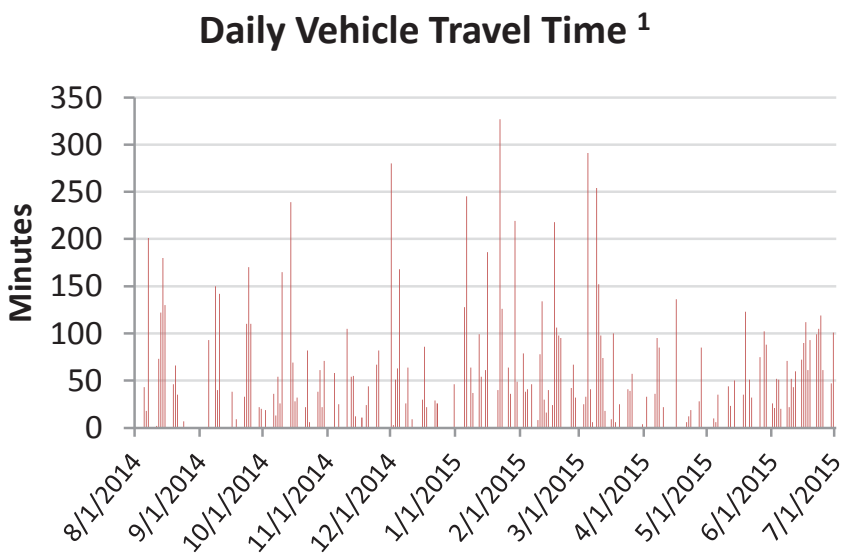

Note 1: Travel time includes all time when vehicle is in the "key on" state, even when the vehicle is not moving

$\begin{array}{ccc}\text { EV Range } & \begin{array}{c}\text { Percentage of } \\ \text { days vehicle } \\ \text { exceeds EV range }\end{array} & \begin{array}{c}\text { Number of days } \\ \text { vehicle exceeds } \\ \text { EV range }\end{array} \\ 63 & 12 \% & 20 \\ 67 & 12 \% & 20 \\ & 11 \% & 18\end{array}$


Vehicle:

Report Period:

Model Year:

Vehicle Make:

Vehicle Model:

Body Type:
5L-G414405H

8/1/2014 00:00:00 - 7/1/2015 00:00:00

2009

Dodge

Caravan

van
Total Number of Days with Driving

Average Number of Trips

Average Trip Distance

Total Number of Trips

Total Distance (miles)

Total Trip Duration (minutes)
184

4.0

17.3

741

3180.6

7311

\section{Distribution of Daily Vehicle Travel Distance}

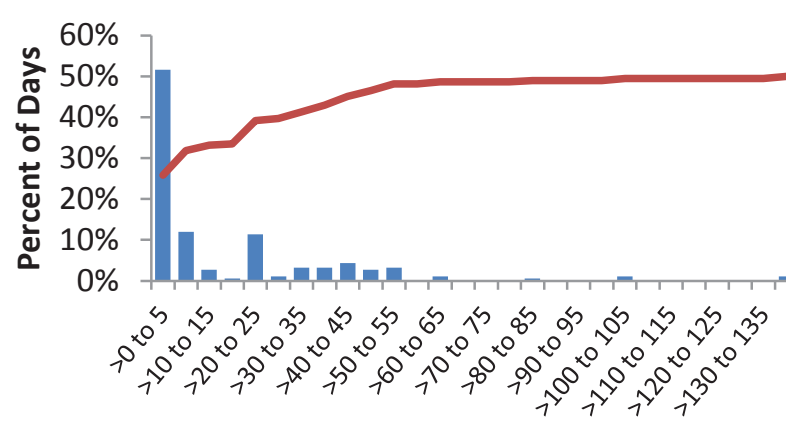

Daily Vehicle Miles Traveled

\section{Daily Vehicle Travel Distance}

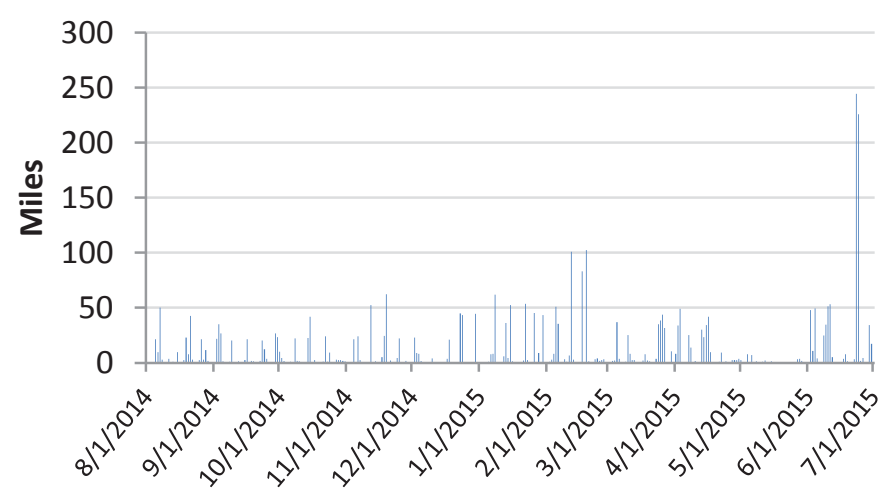

$80 \%$

$60 \%$

$40 \%$

$20 \%$

$0 \%$
Distribution of Daily Vehicle Travel Times ${ }^{1}$

$120 \%$

$100 \%$

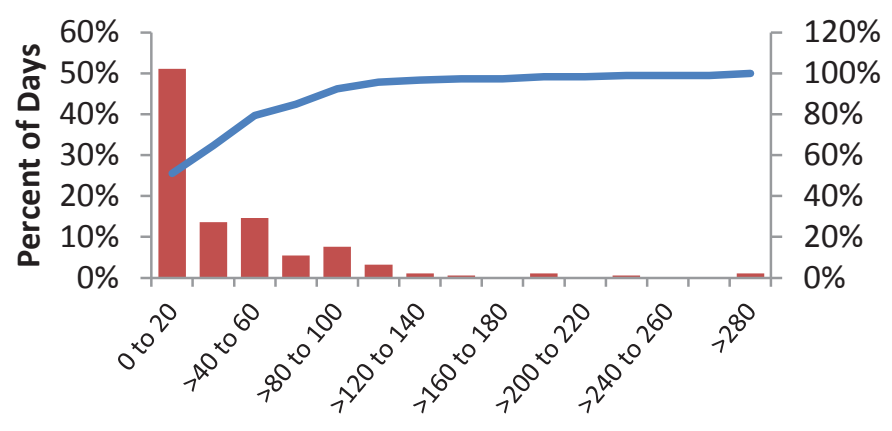

Daily Vehicle Trip Times (min)

\section{Daily Vehicle Travel Time ${ }^{1}$}

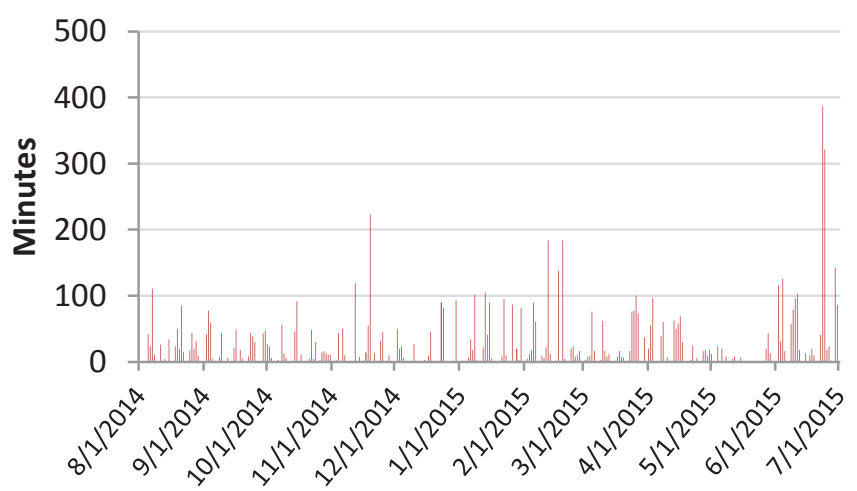

Note 1: Travel time includes all time when vehicle is in the "key on" state, even when the vehicle is not moving

Percentage of Number of days
days vehicle

EV Range

59

63

67 exceeds EV range

$4 \%$

$3 \%$

$3 \%$
EV range

7

5

5 
Vehicle:

Report Period:

Model Year:

Vehicle Make:

Vehicle Model:

Body Type:
5L-G414389H

8/1/2014 00:00:00 - 7/1/2015 00:00:00

2009

Dodge

Caravan

van
Total Number of Days with Driving

Average Number of Trips

Average Trip Distance

Total Number of Trips

Total Distance (miles)

Total Trip Duration (minutes)
194

6.0

35.2

1156

6832.3

16193

\section{Distribution of Daily Vehicle Travel Distance}

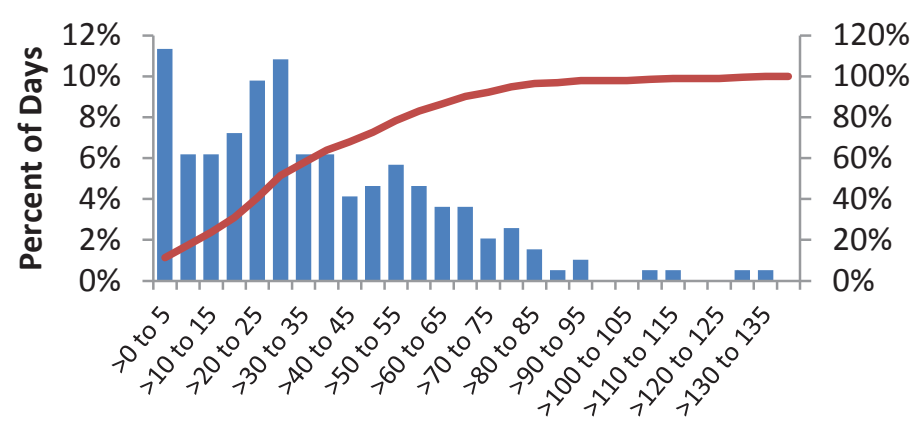

Daily Vehicle Miles Traveled

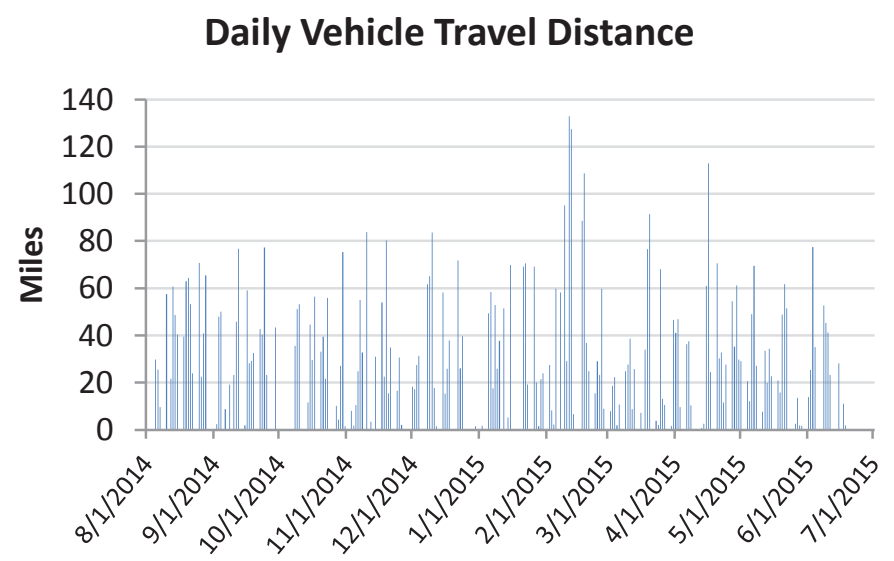

\section{Distribution of Daily Vehicle Travel Times ${ }^{1}$}

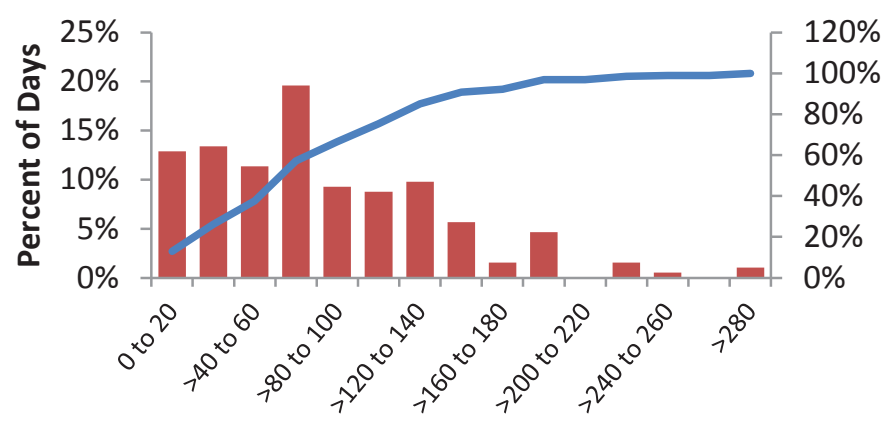

Daily Vehicle Trip Times (min)

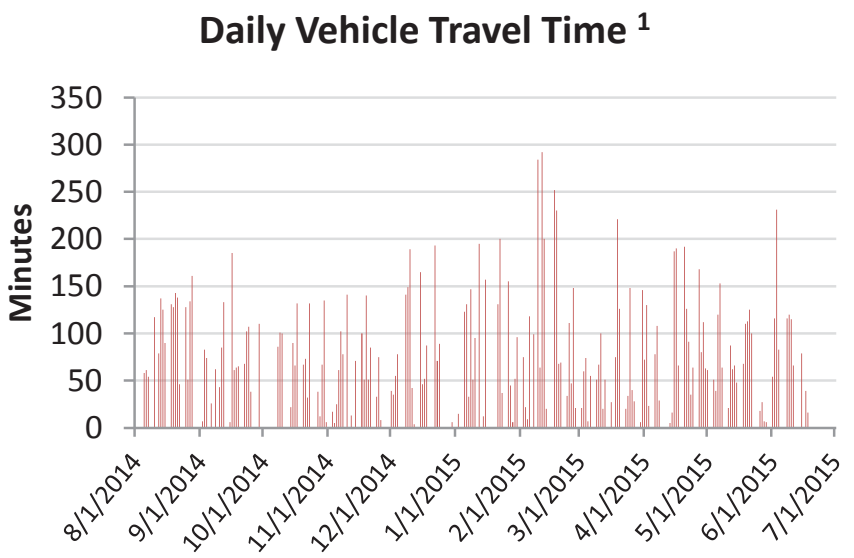

Note 1: Travel time includes all time when vehicle is in the "key on" state, even when the vehicle is not moving

$\begin{array}{ccc}\text { EV Range } & \begin{array}{c}\text { Percentage of } \\ \text { days vehicle } \\ \text { exceeds EV range }\end{array} & \begin{array}{c}\text { Number of days } \\ \text { vehicle exceeds } \\ \text { EV range }\end{array} \\ 59 & 19 \% & 36 \\ 63 & 14 \% & 27 \\ 67 & 12 \% & 24\end{array}$


Vehicle:

Report Period:

Model Year:

Vehicle Make:

Vehicle Model:

Body Type:
5L-G411467F

8/1/2014 00:00:00 - 7/1/2015 00:00:00

2007

Chevrolet

Uplander

van

Total Number of Days with Driving

136

4.5

19.9

616

2706.4

7198

Total Trip Duration (minutes)

Distribution of Daily Vehicle Travel Times ${ }^{1}$

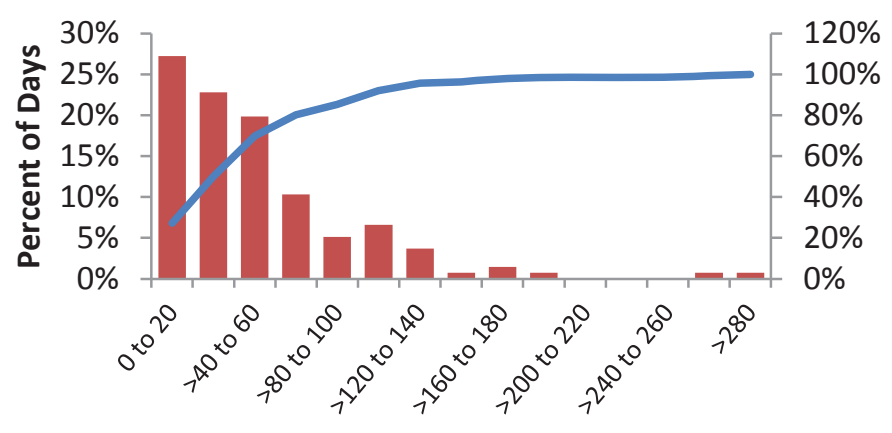

Daily Vehicle Trip Times (min)

Daily Vehicle Miles Traveled

\section{Daily Vehicle Travel Distance}

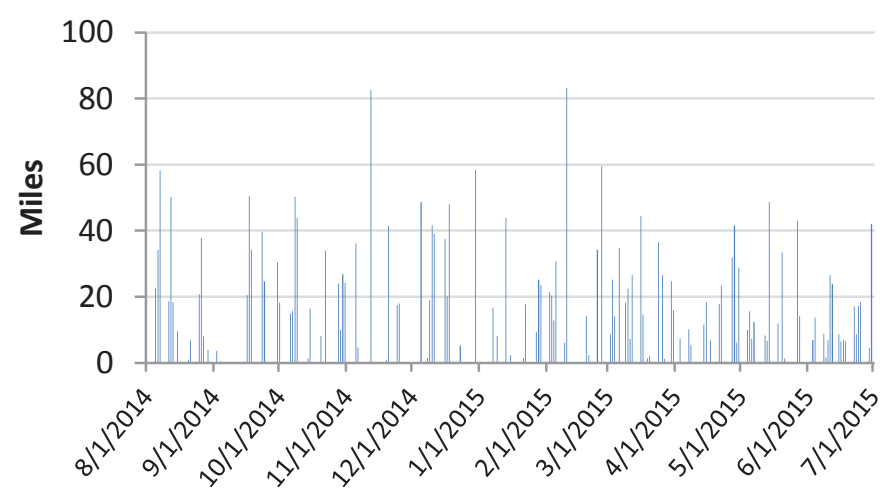

\section{Daily Vehicle Travel Time ${ }^{1}$}

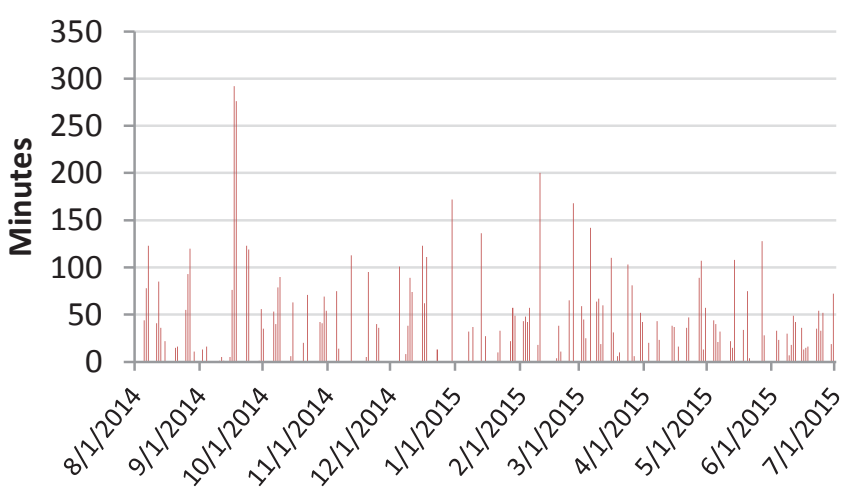

Note 1: Travel time includes all time when vehicle is in the "key on" state, even when the vehicle is not moving

$\begin{array}{cc}\text { Percentage of } & \text { Number of days } \\ \text { days vehicle } & \text { vehicle exceeds }\end{array}$

EV Range

59

63

67 exceeds EV range

$2 \%$

$1 \%$

$1 \%$
EV range

\section{3}

2

2 
Vehicle:

Report Period:

Model Year:

Vehicle Make:

Vehicle Model:

Body Type:
5L-G411466F

8/1/2014 00:00:00 - 7/1/2015 00:00:00

2007

Chevrolet

Uplander

van

Total Number of Days with Driving

Average Number of Trips

Average Trip Distance

Total Number of Trips

Total Distance (miles)

\section{Distribution of Daily Vehicle Travel Distance}

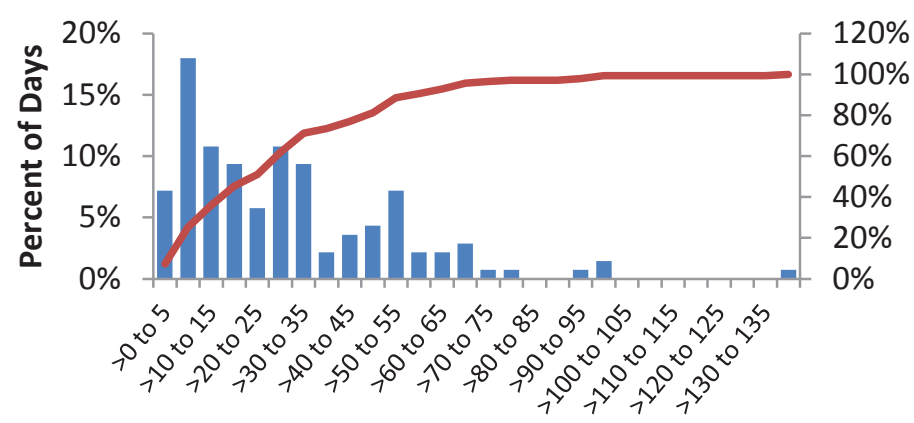

Daily Vehicle Miles Traveled

\section{Daily Vehicle Travel Distance}

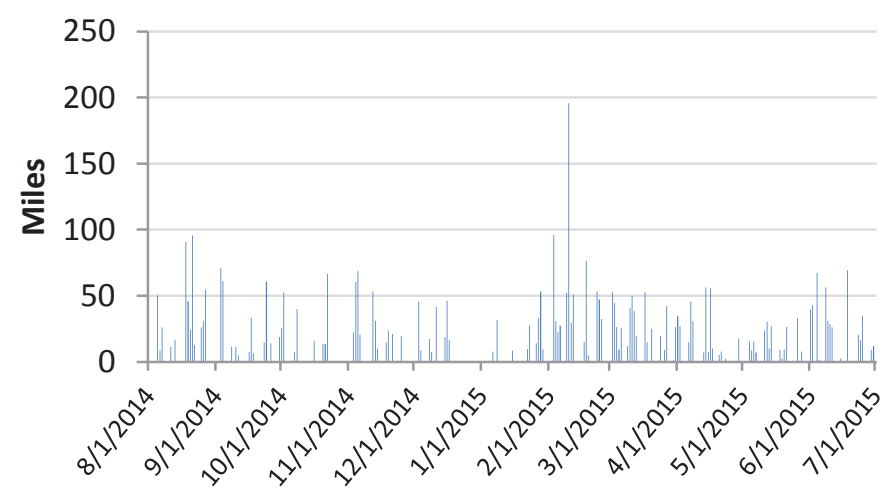

Distribution of Daily Vehicle Travel Times ${ }^{1}$

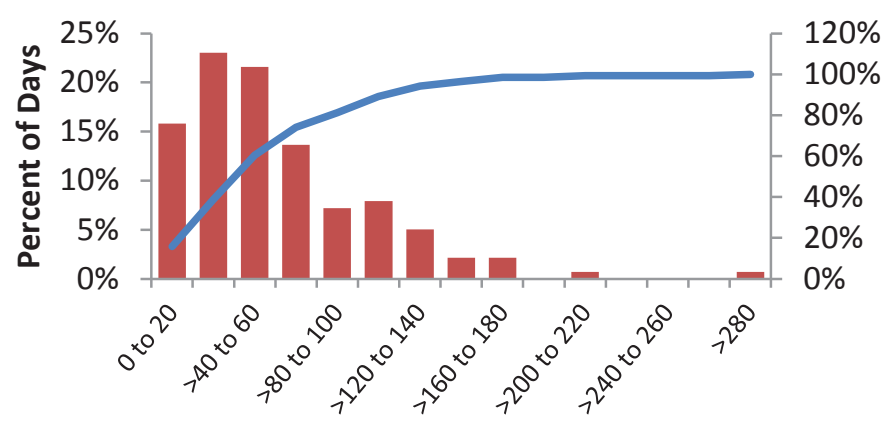

Daily Vehicle Trip Times (min)

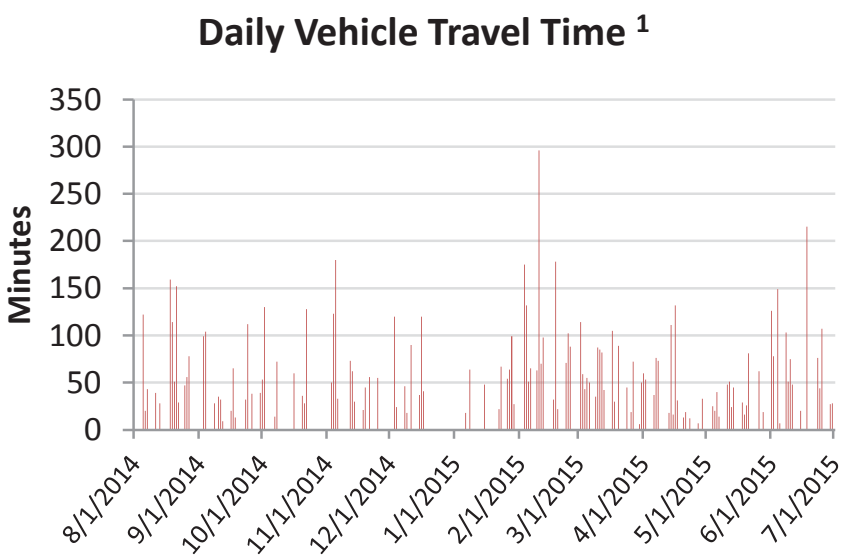

Note 1: Travel time includes all time when vehicle is in the "key on" state, even when the vehicle is not moving

$\begin{array}{cc}\text { Percentage of } & \text { Number of days } \\ \text { days vehicle } & \text { vehicle exceeds }\end{array}$

EV Range

59

63

67 exceeds EV range

$9 \%$

$7 \%$

$6 \%$
EV range

13

10 
Vehicle:

Report Period:

Model Year:

Vehicle Make:

Vehicle Model:

Body Type:
5L-G411447F

8/1/2014 00:00:00 - 7/1/2015 00:00:00

2007

Chevrolet

Uplander

van

Total Number of Days with Driving

Average Number of Trips

5.3

Average Trip Distance

61.9

Total Number of Trips

495

Total Distance (miles)

5752.8

Total Trip Duration (minutes)

11761

\section{Distribution of Daily Vehicle Travel Distance}

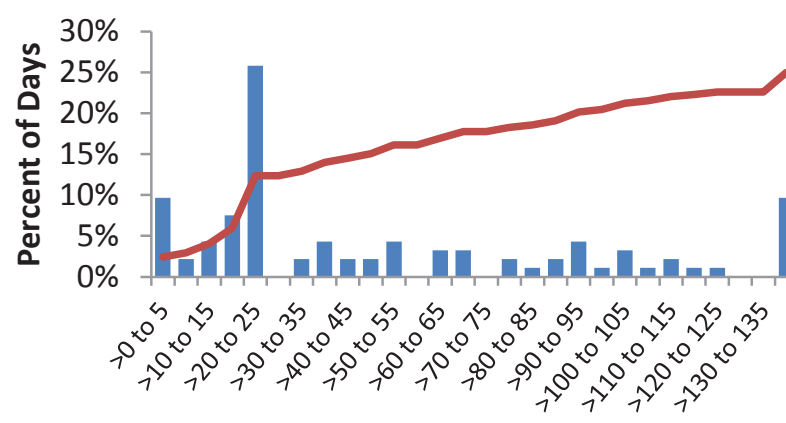

Daily Vehicle Miles Traveled

\section{Daily Vehicle Travel Distance}

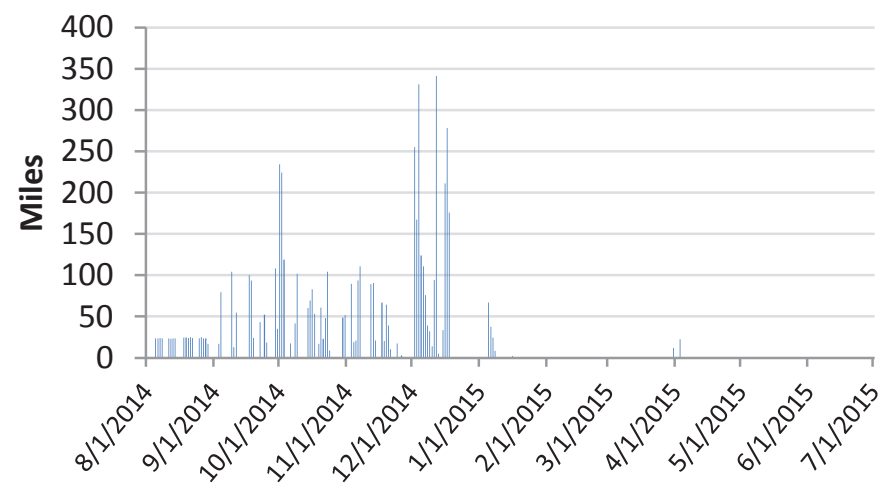

Distribution of Daily Vehicle Travel Times ${ }^{1}$

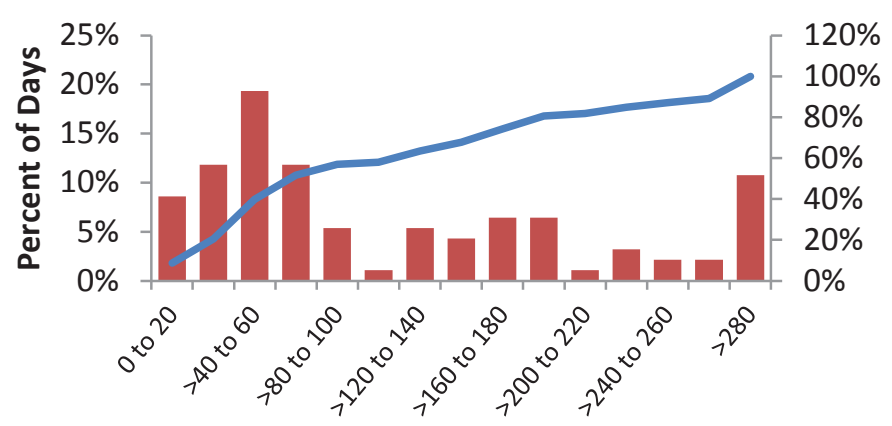

Daily Vehicle Trip Times (min)

\section{Daily Vehicle Travel Time ${ }^{1}$}

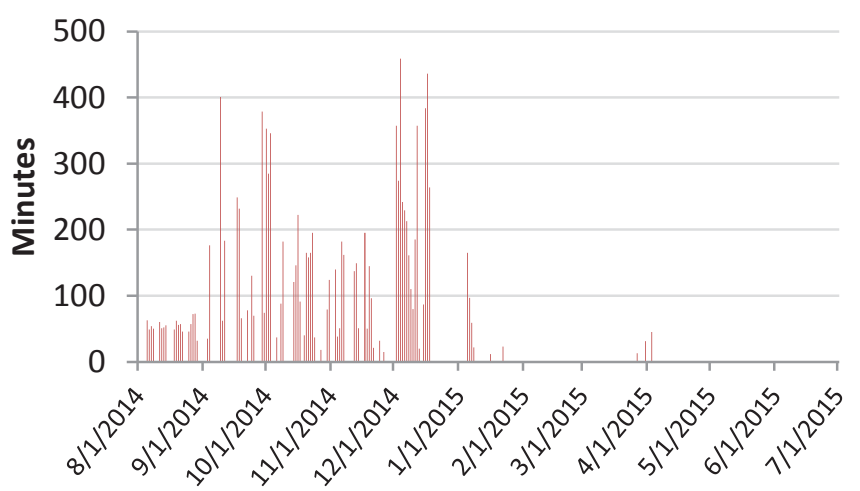

Note 1: Travel time includes all time when vehicle is in the "key on" state, even when the vehicle is not moving

$\begin{array}{cc}\text { Percentage of } & \text { Number of days } \\ \text { days vehicle } & \text { vehicle exceeds }\end{array}$

EV Range

59

63

67 exceeds EV range

$35 \%$

$33 \%$

$30 \%$
EV range

33

31

28 
Vehicle:

Report Period:

Model Year:

Vehicle Make:

Vehicle Model:

Body Type:
5L-G411436F

8/1/2014 00:00:00 - 7/1/2015 00:00:00

2007

Chevrolet

Uplander

van

Total Number of Days with Driving

255

6.9

11.3

1772

2878.5

12531

Total Trip Duration (minutes)

Distribution of Daily Vehicle Travel Times ${ }^{1}$

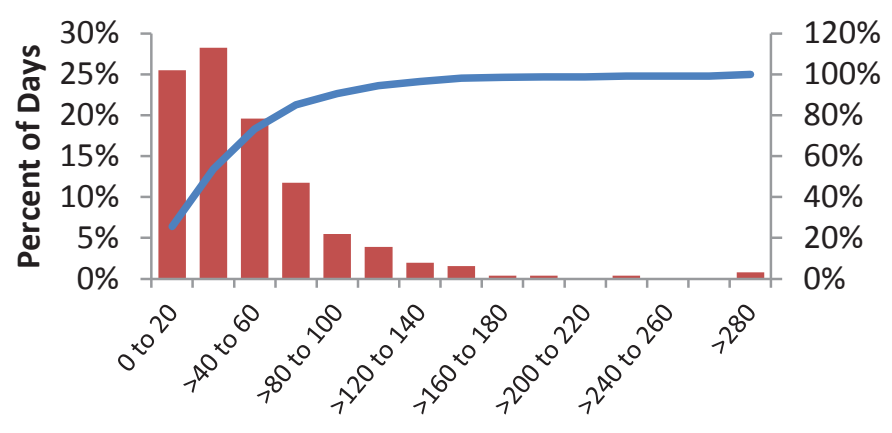

Daily Vehicle Trip Times (min)

Daily Vehicle Miles Traveled
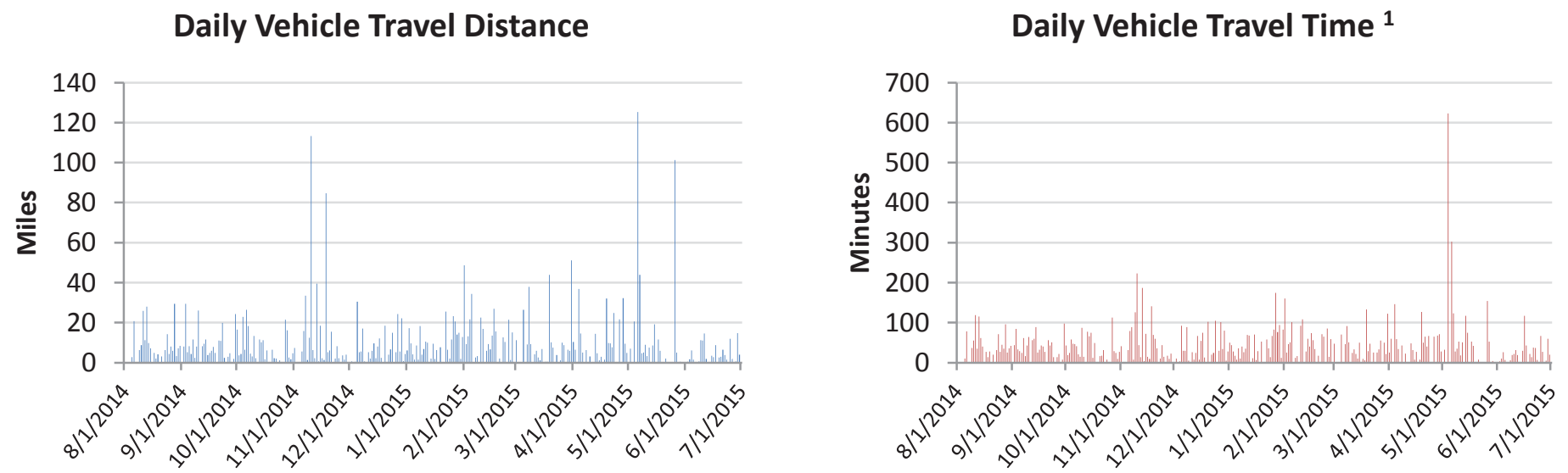

Note 1: Travel time includes all time when vehicle is in the "key on" state, even when the vehicle is not moving

EV Range

59

63

67

$$
\begin{array}{cc}
\begin{array}{c}
\text { Percentage of } \\
\text { Nays vehicle }
\end{array} & \text { vehicle exceeds }
\end{array}
$$

exceeds EV range EV range

$2 \%$

$2 \%$

$2 \%$
4

4

4 
Vehicle:

Report Period:

Model Year:

Vehicle Make:

Vehicle Model:

Body Type:
5L-G410514G

8/1/2014 00:00:00 - 7/1/2015 00:00:00

2008

Chevrolet

Uplander LS

van
Total Number of Days with Driving

Average Number of Trips

Average Trip Distance

Total Number of Trips

Total Distance (miles)

Total Trip Duration (minutes)
26

5.6

52.9

146

1375.5

3221

\section{Distribution of Daily Vehicle Travel Distance}

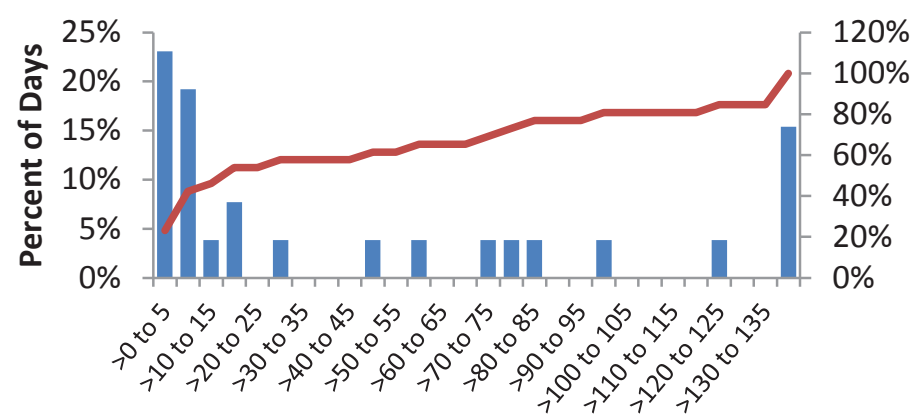

Daily Vehicle Miles Traveled

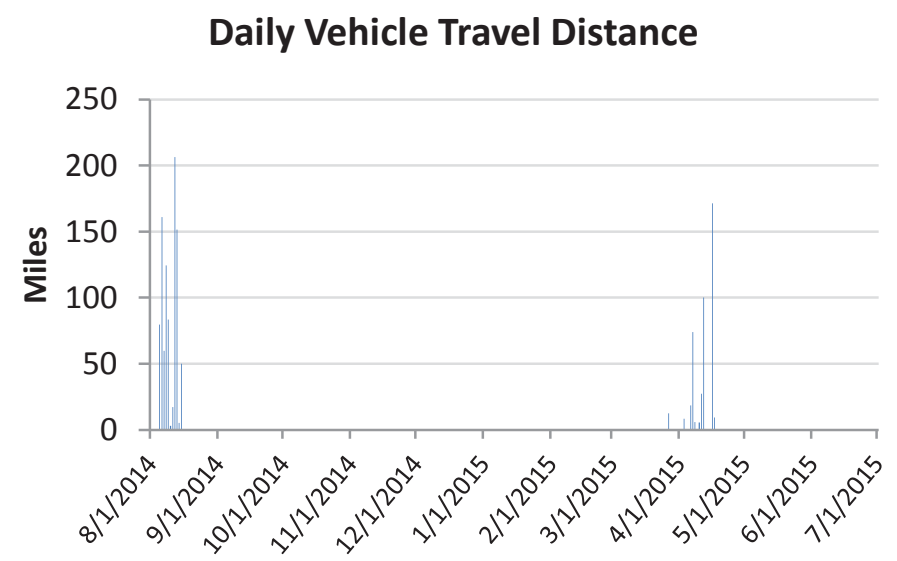

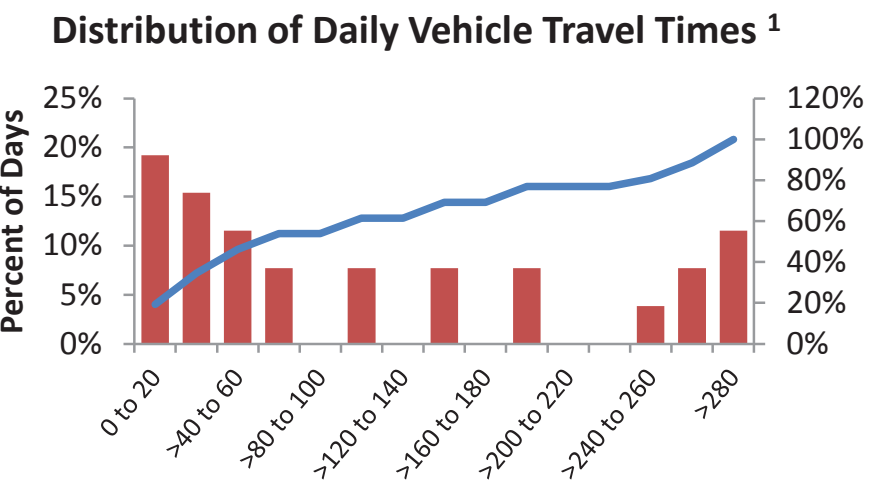

Daily Vehicle Trip Times ( $\mathrm{min})$

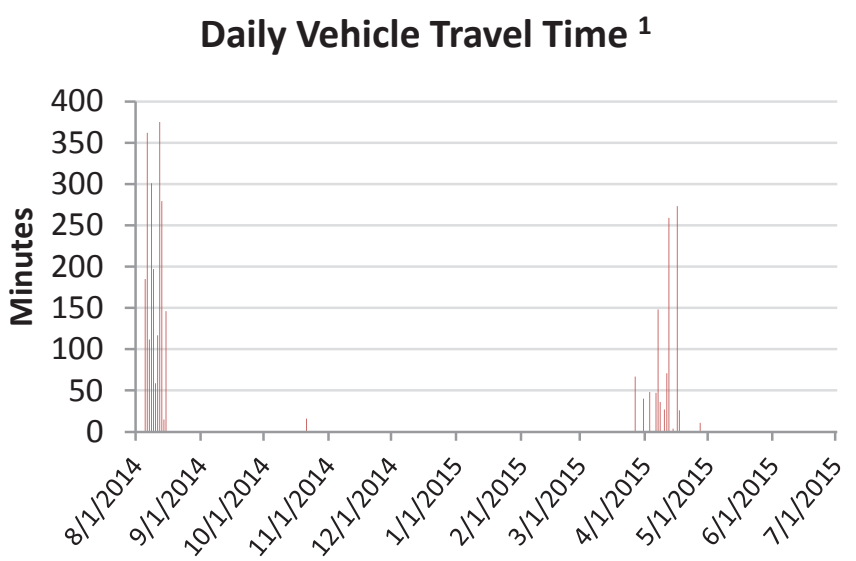

Note 1: Travel time includes all time when vehicle is in the "key on" state, even when the vehicle is not moving

$\begin{array}{ccc}\text { EV Range } & \begin{array}{c}\text { Percentage of } \\ \text { days vehicle } \\ \text { exceeds EV range }\end{array} & \begin{array}{c}\text { Number of days } \\ \text { vehicle exceeds } \\ \text { EV range }\end{array} \\ 63 & 38 \% & 10 \\ 67 & 35 \% & 9 \\ 63 & 35 \% & 9\end{array}$


Vehicle:

Report Period:

Model Year:

Vehicle Make:

Vehicle Model:

Body Type:
5L-G410489G

8/1/2014 00:00:00 - 7/1/2015 00:00:00

2008

Chevrolet

Uplander LS

van
Total Number of Days with Driving

Average Number of Trips

Average Trip Distance

Total Number of Trips

Total Distance (miles)

Total Trip Duration (minutes)
174

5.3

40.7

915

7081.9

18170

\section{Distribution of Daily Vehicle Travel Distance}

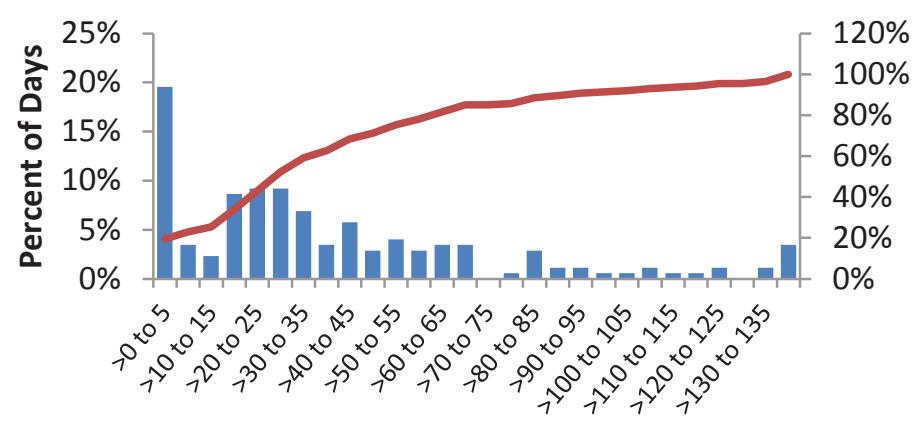

Daily Vehicle Miles Traveled

\section{Daily Vehicle Travel Distance}

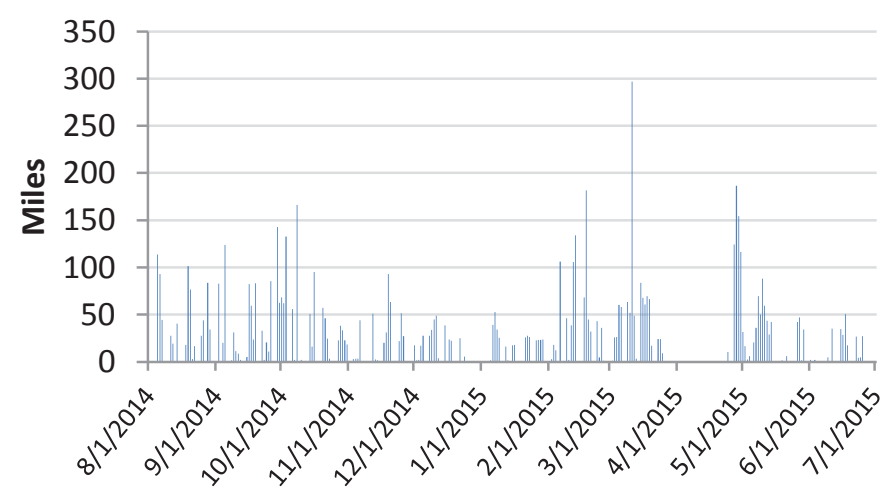

Distribution of Daily Vehicle Travel Times ${ }^{1}$

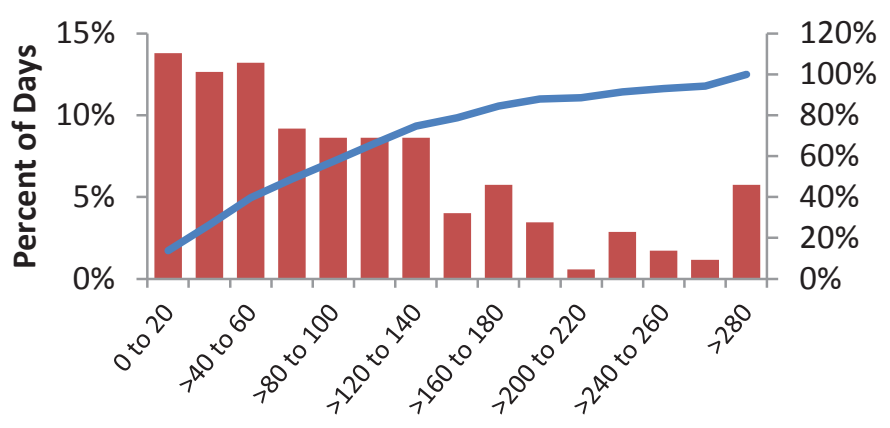

Daily Vehicle Trip Times ( $\mathrm{min})$

\section{Daily Vehicle Travel Time ${ }^{1}$}

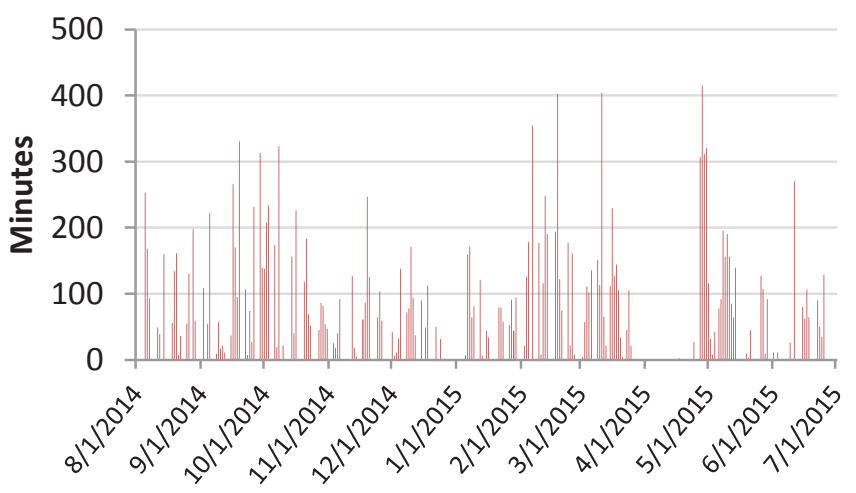

Note 1: Travel time includes all time when vehicle is in the "key on" state, even when the vehicle is not moving

$\begin{array}{cc}\text { Percentage of } & \text { Number of days } \\ \text { days vehicle } & \text { vehicle exceeds }\end{array}$

EV Range

59

63

67 exceeds EV range

$23 \%$

$20 \%$

$18 \%$
EV range

40

34

31 
Vehicle:

Report Period:

Model Year:

Vehicle Make:

Vehicle Model:

Body Type:
5L-G410487G

8/1/2014 00:00:00 - 7/1/2015 00:00:00

2008

Chevrolet

Uplander LS

van

Total Number of Days with Driving

Average Number of Trips

Average Trip Distance

Total Number of Trips

710

Total Distance (miles)

3507.5

Total Trip Duration (minutes)

\section{Distribution of Daily Vehicle Travel Distance}

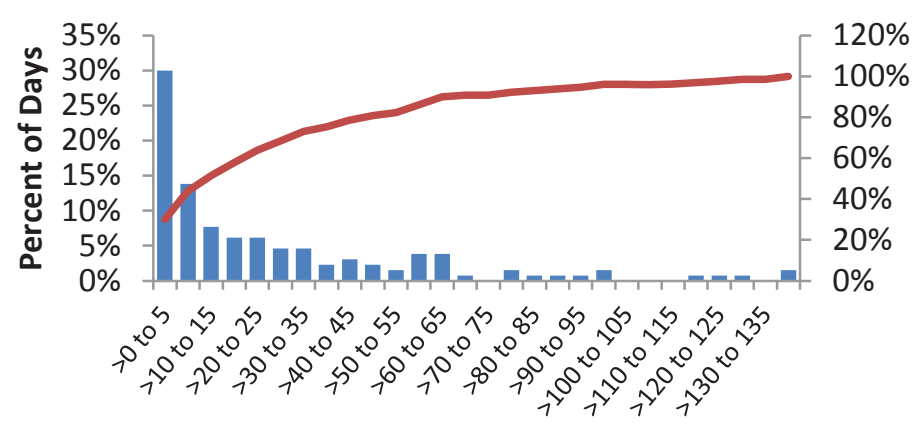

Daily Vehicle Miles Traveled

\section{Daily Vehicle Travel Distance}

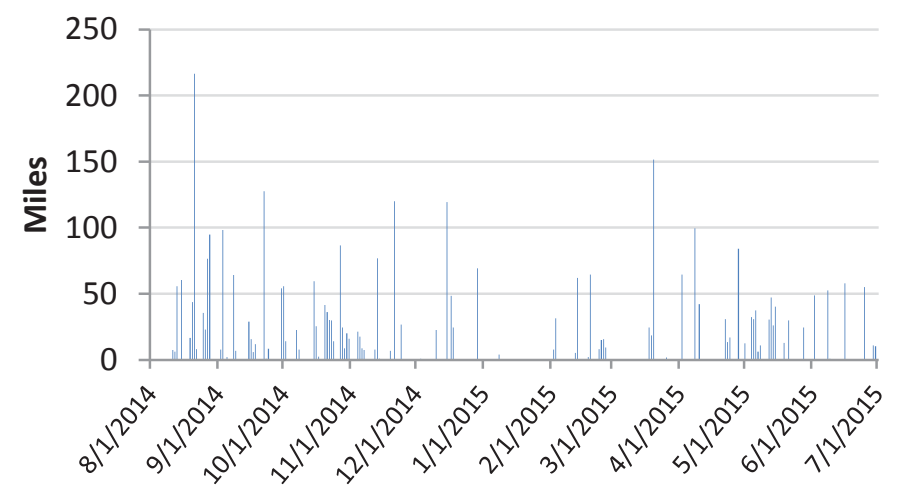

\section{Distribution of Daily Vehicle Travel Times ${ }^{1}$}

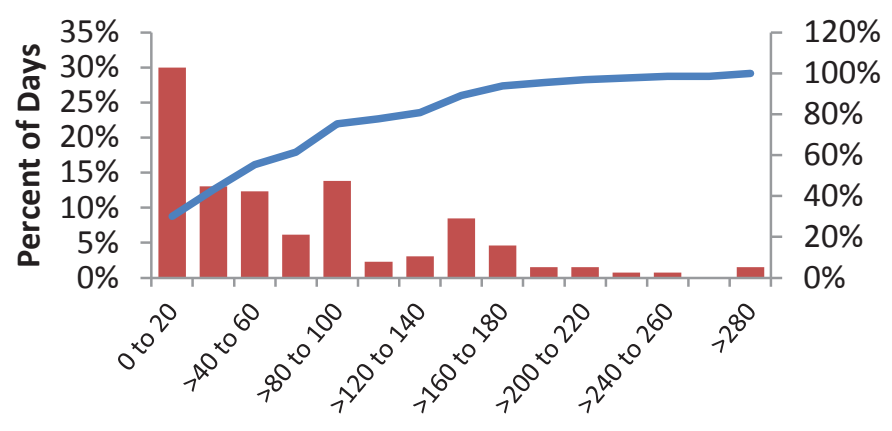

Daily Vehicle Trip Times (min)

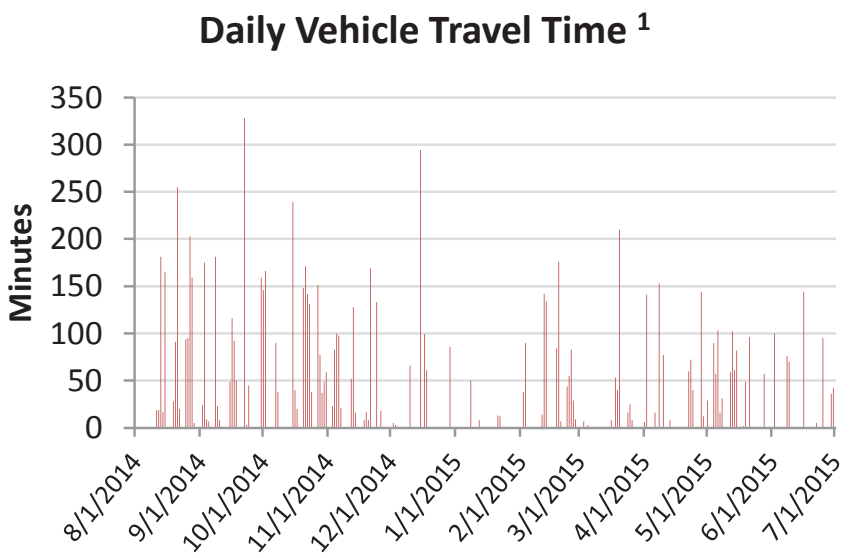

Note 1: Travel time includes all time when vehicle is in the "key on" state, even when the vehicle is not moving

$\begin{array}{cc}\text { Percentage of } & \text { Number of days } \\ \text { days vehicle } & \text { vehicle exceeds }\end{array}$

EV Range

59

63

67 exceeds EV range

$15 \%$

$12 \%$

$10 \%$
EV range

19

16

13 
Vehicle:

Report Period:

Model Year:

Vehicle Make:

Vehicle Model:

Body Type:
5L-G136271L

8/1/2014 00:00:00 - 7/1/2015 00:00:00

2012

FORD

Focus

sedan
Total Number of Days with Driving

Average Number of Trips

Average Trip Distance

Total Number of Trips

Total Distance (miles)

Total Trip Duration (minutes)
72

2.9

11.9

209

857.3

2235

\section{Distribution of Daily Vehicle Travel Distance}

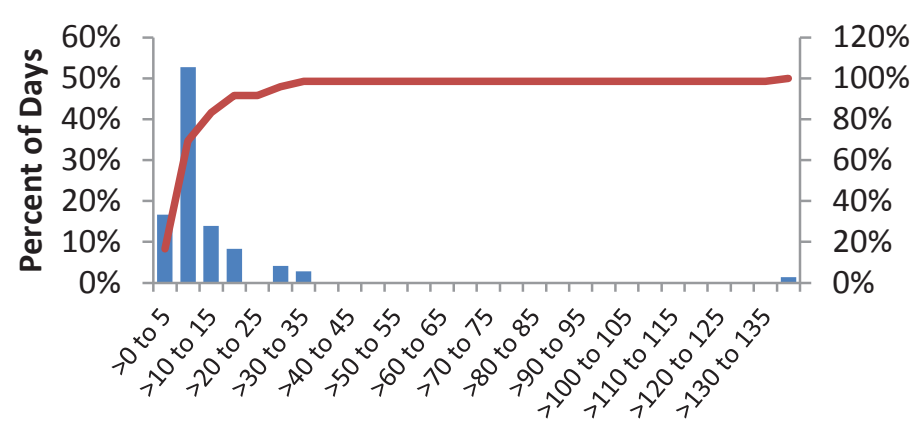

Daily Vehicle Miles Traveled

\section{Daily Vehicle Travel Distance}

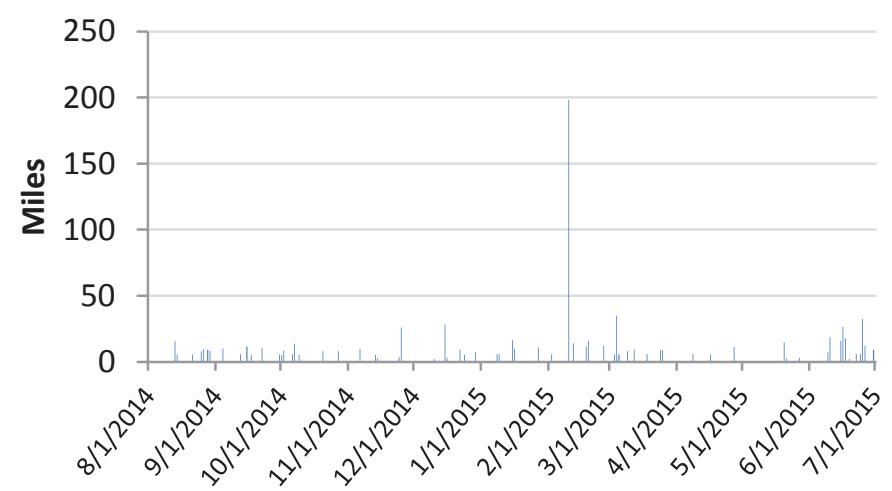

Distribution of Daily Vehicle Travel Times ${ }^{1}$

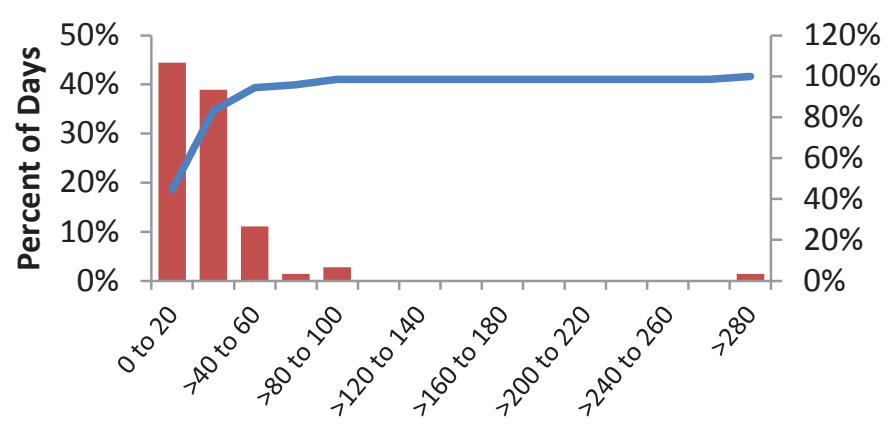

Daily Vehicle Trip Times (min)

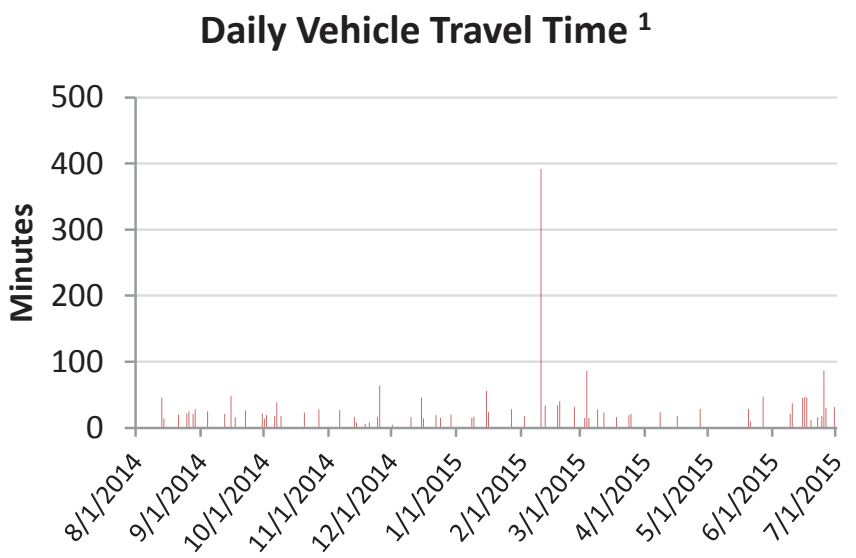

Note 1: Travel time includes all time when vehicle is in the "key on" state, even when the vehicle is not moving

$\begin{array}{cc}\text { Percentage of } & \text { Number of days } \\ \text { days vehicle } & \text { vehicle exceeds }\end{array}$

EV Range

59

63

67 exceeds EV range

$1 \%$

$1 \%$

$1 \%$
EV range

\section{1}

1

1 
Vehicle:

Report Period:

Model Year:

Vehicle Make:

Vehicle Model:

Body Type:
5L-G109773K

8/1/2014 00:00:00 - 7/1/2015 00:00:00

2011

FORD

Fusion Hybrid

sedan
Total Number of Days with Driving

1

0.0

0.0

Average Trip Distance

Total Number of Trips

Total Distance (miles)

Total Trip Duration (minutes)
Distribution of Daily Vehicle Travel Times ${ }^{1}$

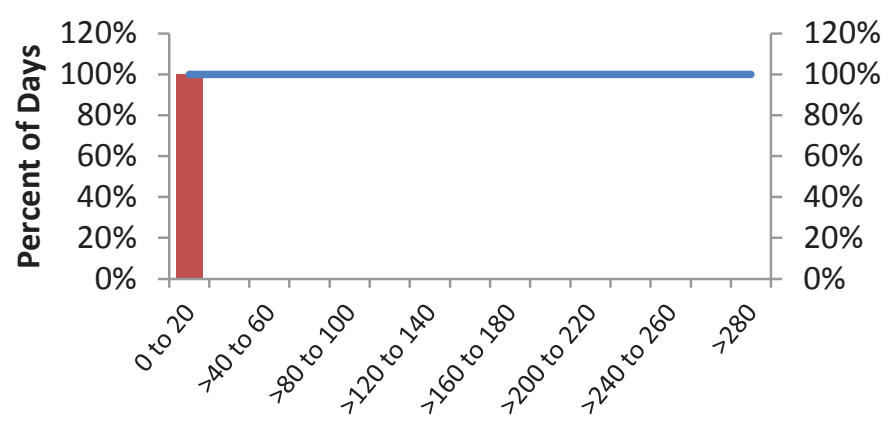

Daily Vehicle Trip Times (min)

\section{Daily Vehicle Travel Time ${ }^{1}$}

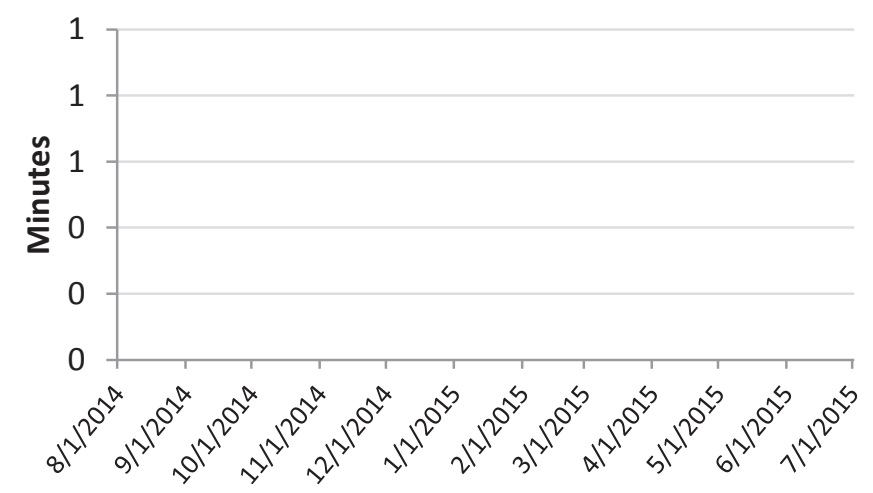

Note 1: Travel time includes all time when vehicle is in the "key on" state, even when the vehicle is not moving

Percentage of Number of days
days vehicle

EV Range

59

63

67 exceeds EV range

$0 \%$

$0 \%$

$0 \%$
EV range

0

0

0 
Vehicle:

Report Period:

Model Year:

Vehicle Make:

Vehicle Model:

Body Type:

Total Number of Days with Driving

Average Number of Trips

Average Trip Distance

Total Number of Trips

Total Distance (miles)

Total Trip Duration (minutes)
5L-G109771K

8/1/2014 00:00:00 - 7/1/2015 00:00:00

2011

FORD

Fusion Hybrid

sedan

\section{Distribution of Daily Vehicle Travel Distance}

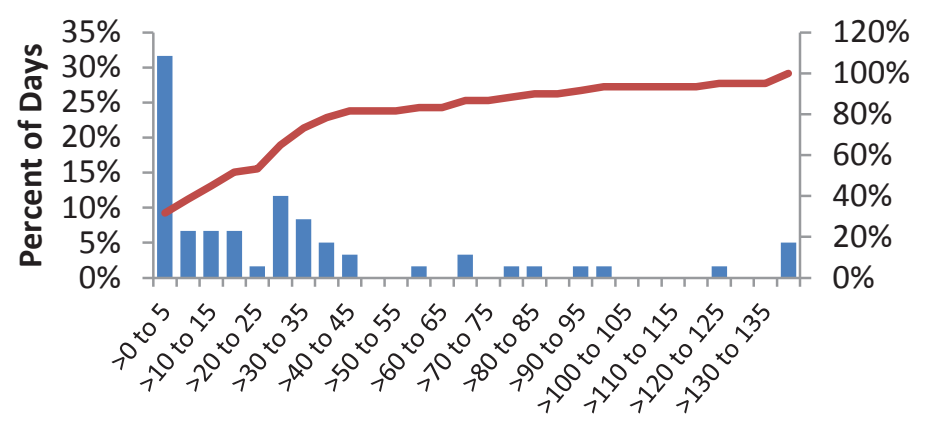

Daily Vehicle Miles Traveled

\section{Daily Vehicle Travel Distance}

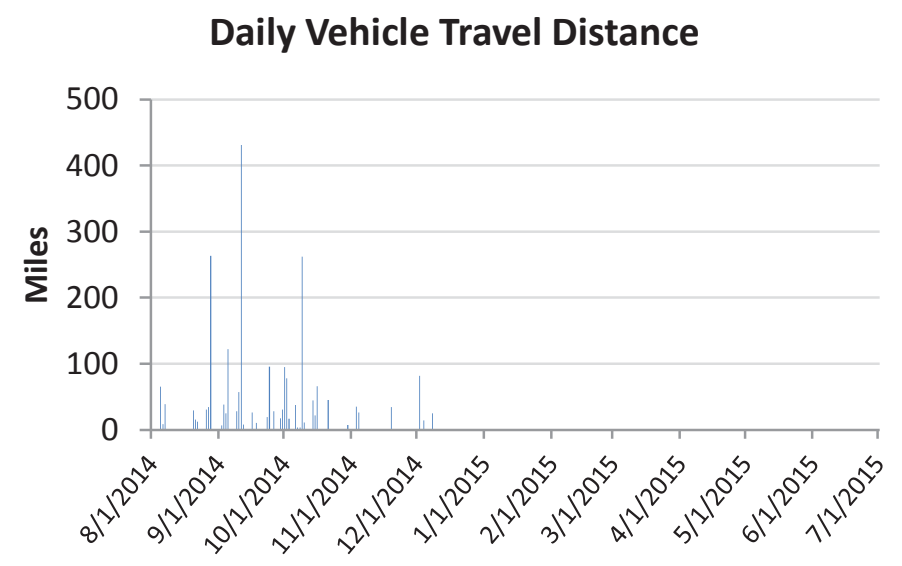

60

5.3

39.3

323

2356.1

4530
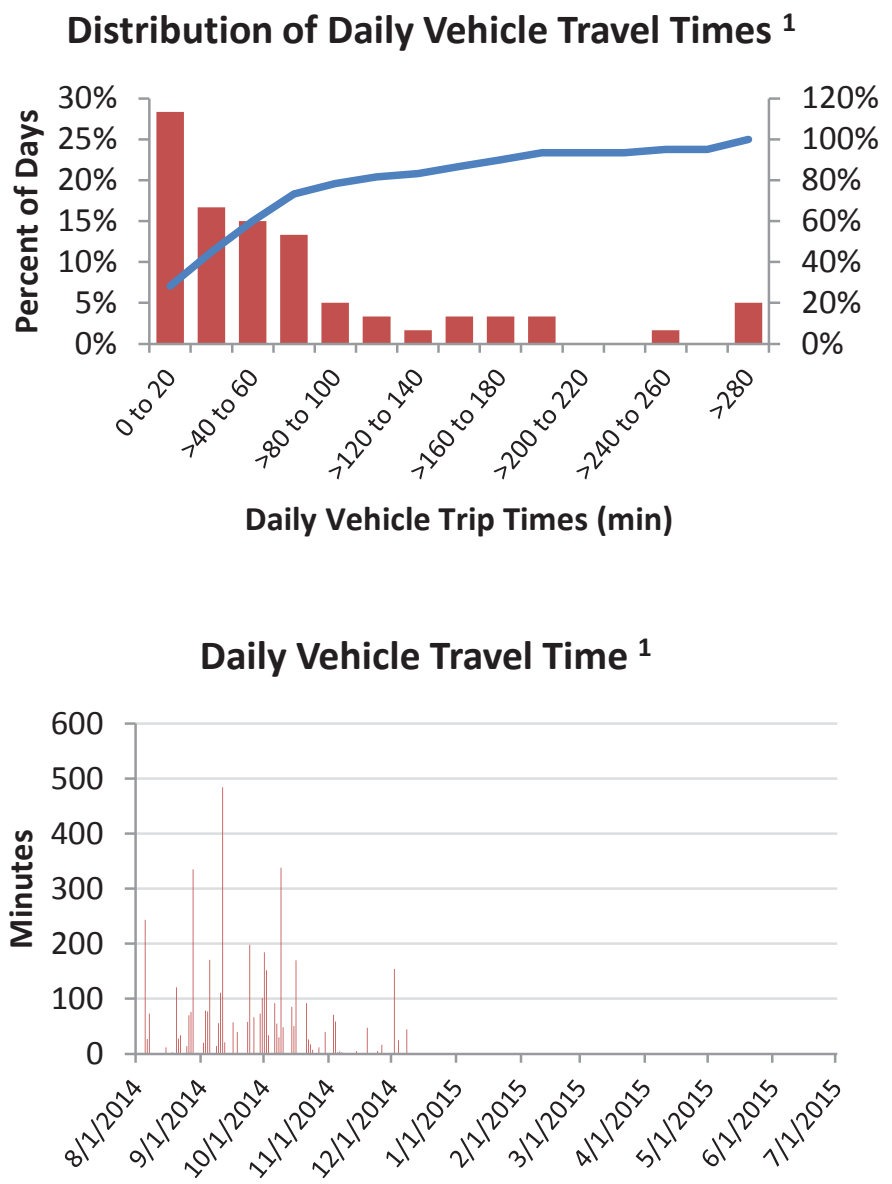

Note 1: Travel time includes all time when vehicle is in the "key on" state, even when the vehicle is not moving

$\begin{array}{ccc}\text { EV Range } & \begin{array}{c}\text { Percentage of } \\ \text { days vehicle } \\ \text { exceeds EV range }\end{array} & \begin{array}{c}\text { Number of days } \\ \text { vehicle exceeds } \\ \text { EV range }\end{array} \\ 59 & 17 \% & 10 \\ 63 & 17 \% & 10 \\ 67 & 13 \% & 8\end{array}$


Vehicle:

Report Period:

Model Year:

Vehicle Make:

Vehicle Model:

Body Type:

Total Number of Days with Driving

Average Number of Trips

Average Trip Distance

Total Number of Trips

Total Distance (miles)

Total Trip Duration (minutes)
5L-G109769K

8/1/2014 00:00:00 - 7/1/2015 00:00:00

2011

FORD

Fusion Hybrid

sedan
232

5.1

34.7

1180

8040.8

15477

\section{Distribution of Daily Vehicle Travel Distance}

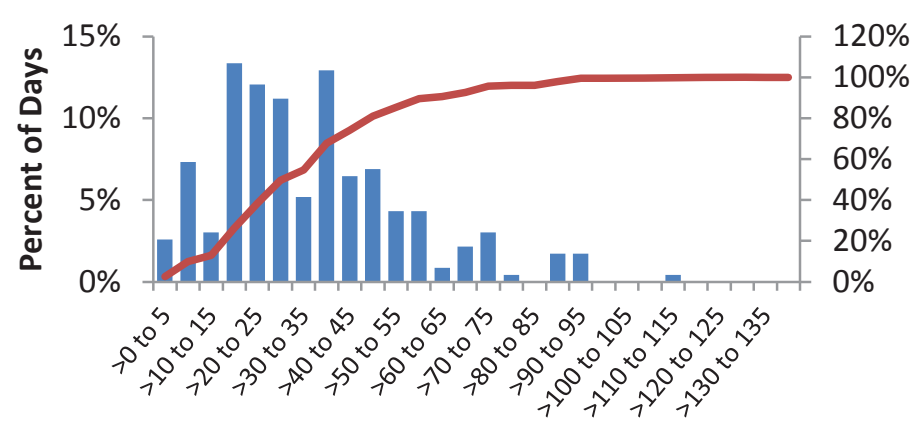

Daily Vehicle Miles Traveled

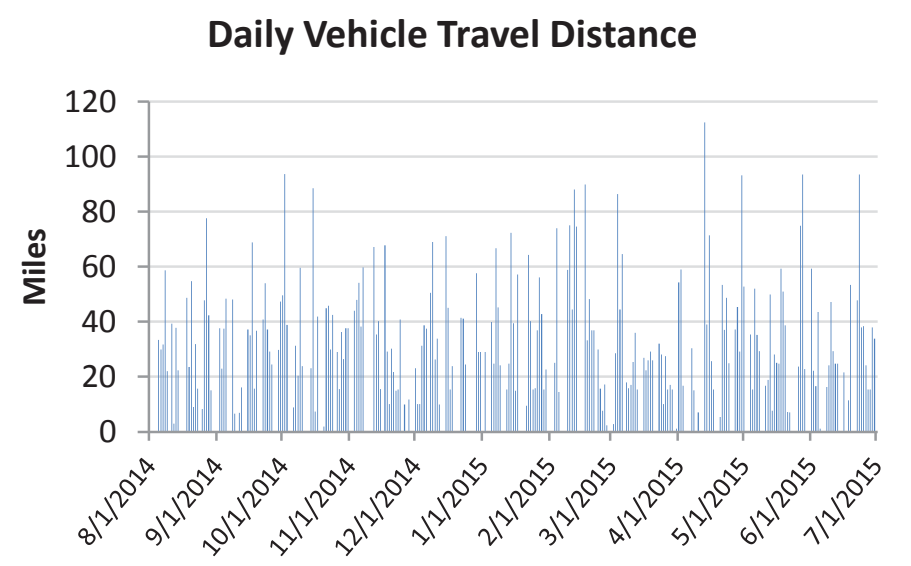

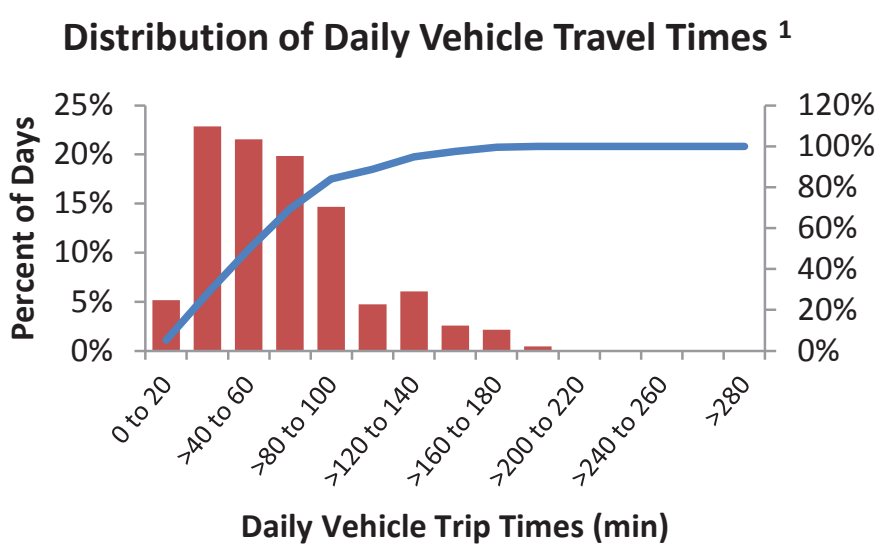

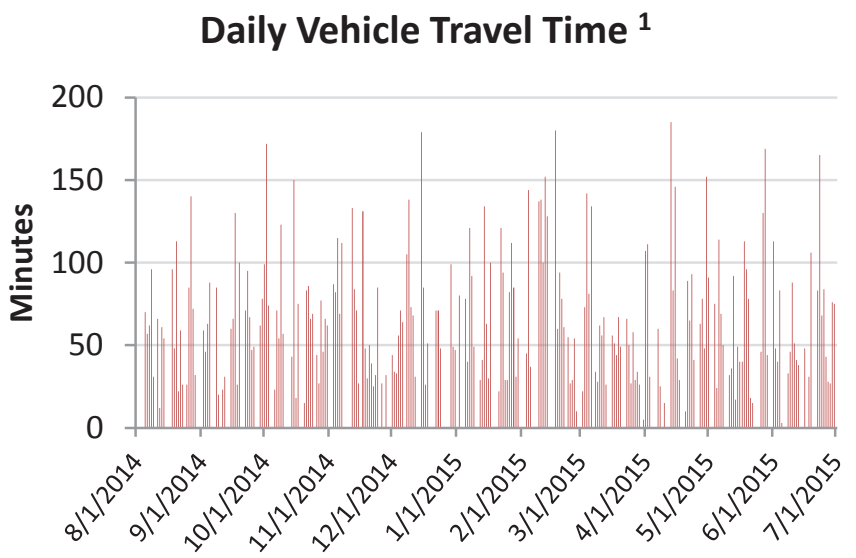

Note 1: Travel time includes all time when vehicle is in the "key on" state, even when the vehicle is not moving

$\begin{array}{ccc}\text { EV Range } & \begin{array}{c}\text { Percentage of } \\ \text { days vehicle } \\ \text { exceeds EV range }\end{array} & \begin{array}{c}\text { Number of days } \\ \text { vehicle exceeds } \\ \text { EV range }\end{array} \\ 69 & 12 \% & 28 \\ 63 & 10 \% & 24 \\ 67 & 9 \% & 21\end{array}$


Vehicle:

Report Period:

Model Year:

Vehicle Make:

Vehicle Model:

Body Type:

Total Number of Days with Driving

Average Number of Trips

Average Trip Distance

Total Number of Trips

Total Distance (miles)

Total Trip Duration (minutes)
5L-G109768K

8/1/2014 00:00:00 - 7/1/2015 00:00:00

2011

FORD

Fusion Hybrid

sedan

\section{Distribution of Daily Vehicle Travel Distance}

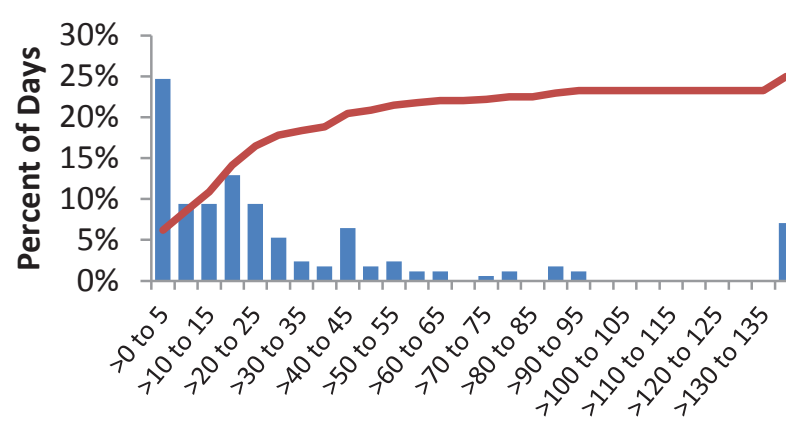

Daily Vehicle Miles Traveled

\section{Daily Vehicle Travel Distance}
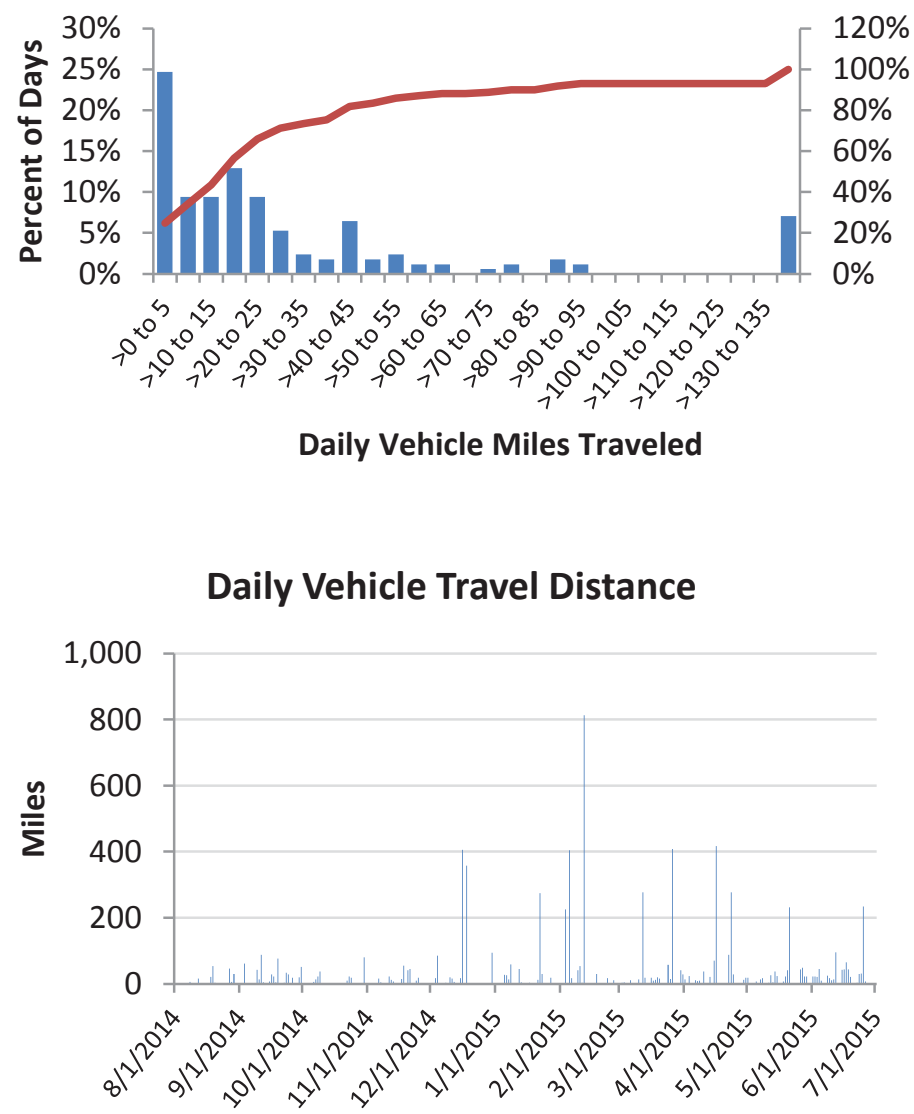

$20 \%$
$0 \%$

\section{.} .
170

17.9

45.2

3036

7681.6

9029

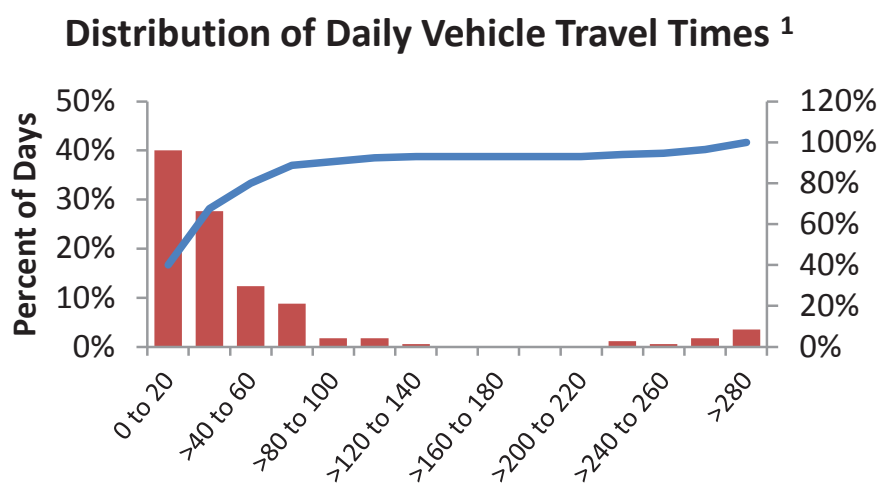

Daily Vehicle Trip Times (min)

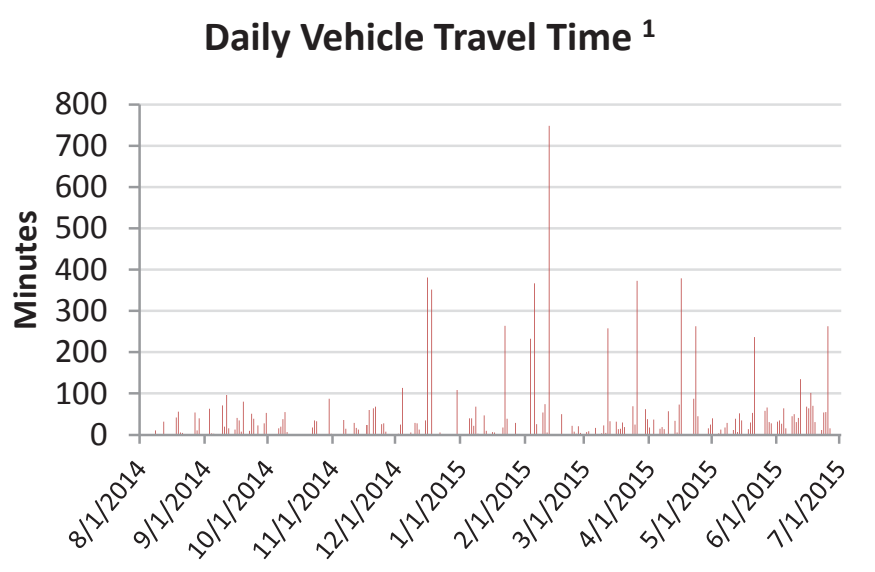

Note 1: Travel time includes all time when vehicle is in the "key on" state, even when the vehicle is not moving

$\begin{array}{ccc}\text { EV Range } & \begin{array}{c}\text { Percentage of } \\ \text { days vehicle } \\ \text { exceeds EV range }\end{array} & \begin{array}{c}\text { Number of days } \\ \text { vehicle exceeds } \\ \text { EV range }\end{array} \\ 59 & 13 \% & 22 \\ 63 & 12 \% & 21 \\ 67 & 12 \% & 20\end{array}$


Vehicle:

Report Period:

Model Year:

Vehicle Make:

Vehicle Model:

Body Type:

Total Number of Days with Driving

Average Number of Trips

Average Trip Distance

Total Number of Trips

Total Distance (miles)

Total Trip Duration (minutes)
5L-G109766K

8/1/2014 00:00:00 - 7/1/2015 00:00:00

2011

FORD

Fusion Hybrid

sedan

\section{Distribution of Daily Vehicle Travel Distance}

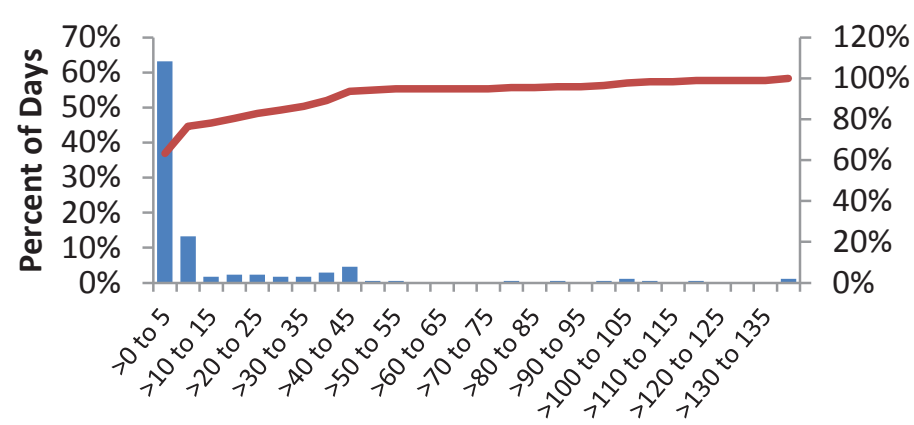

Daily Vehicle Miles Traveled

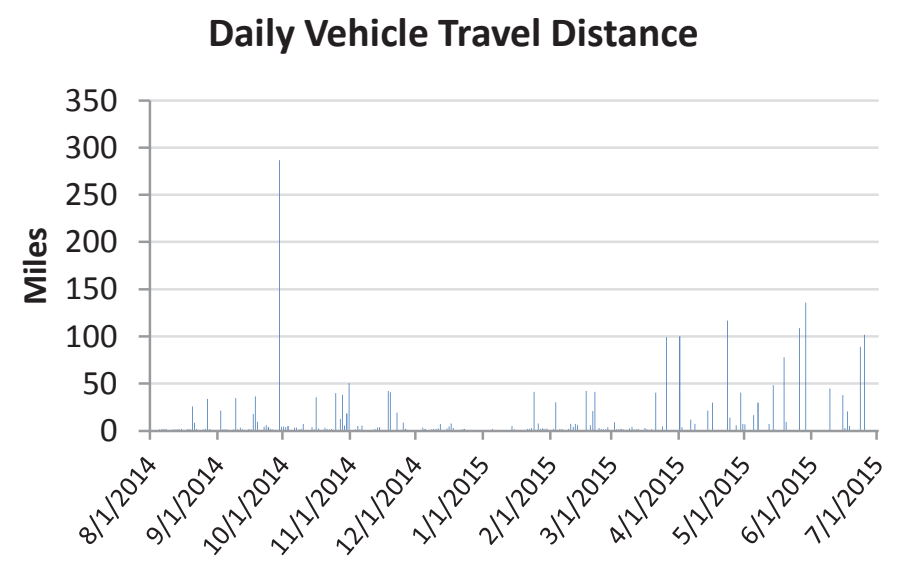

174

4.4

14.4

768

2505.0

6115

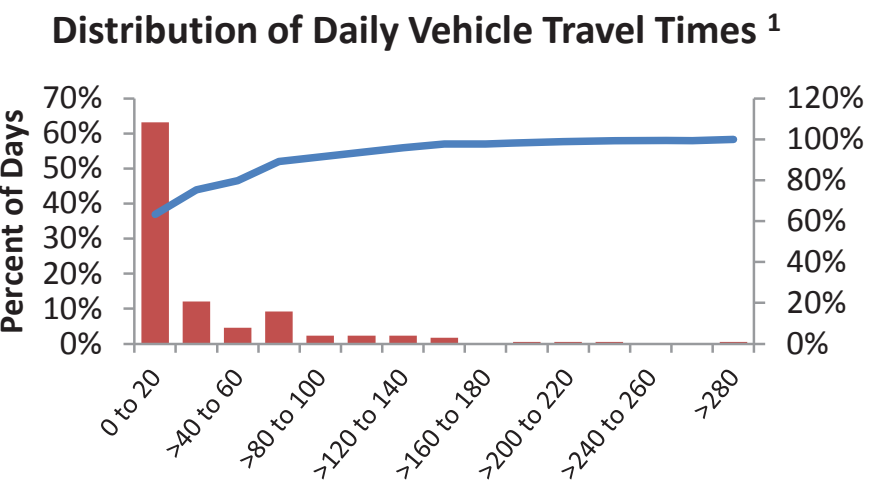

Daily Vehicle Trip Times (min)

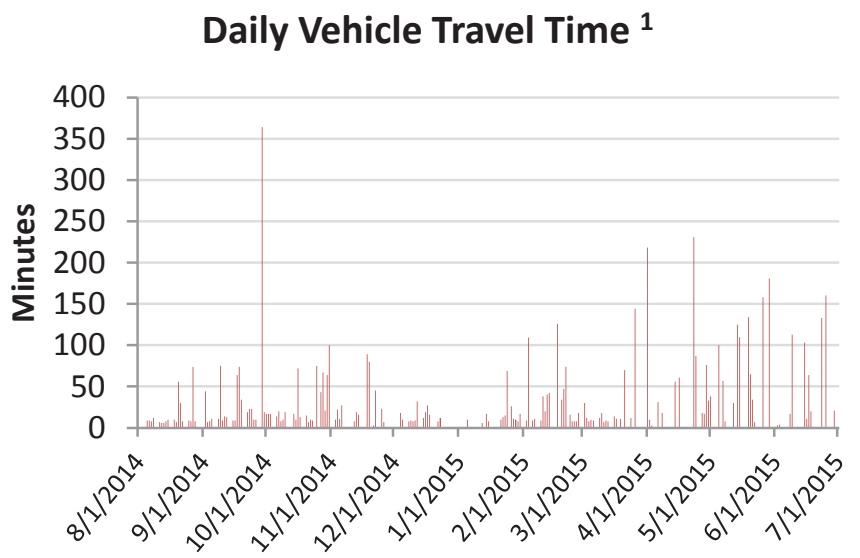

Note 1: Travel time includes all time when vehicle is in the "key on" state, even when the vehicle is not moving

$\begin{array}{cc}\text { Percentage of } & \text { Number of days } \\ \text { days vehicle } & \text { vehicle exceeds }\end{array}$

EV Range

59

63

67 exceeds EV range

$5 \%$

$5 \%$

$5 \%$
EV range

9

9

9 
Vehicle:

Report Period:

Model Year:

Vehicle Make:

Vehicle Model:

Body Type:

Total Number of Days with Driving

Average Number of Trips

Average Trip Distance

Total Number of Trips

Total Distance (miles)

Total Trip Duration (minutes)
5L-G107279L

8/1/2014 00:00:00 - 7/1/2015 00:00:00

2011

Chevy

Malibu

sedan

\section{Distribution of Daily Vehicle Travel Distance}

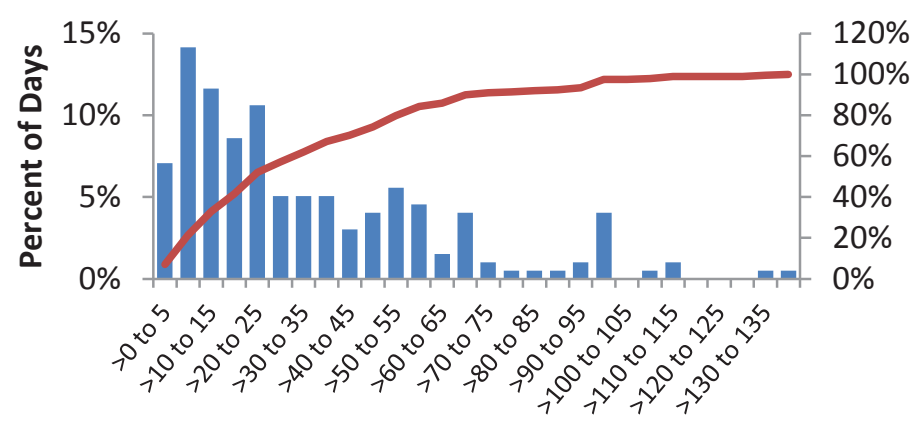

Daily Vehicle Miles Traveled

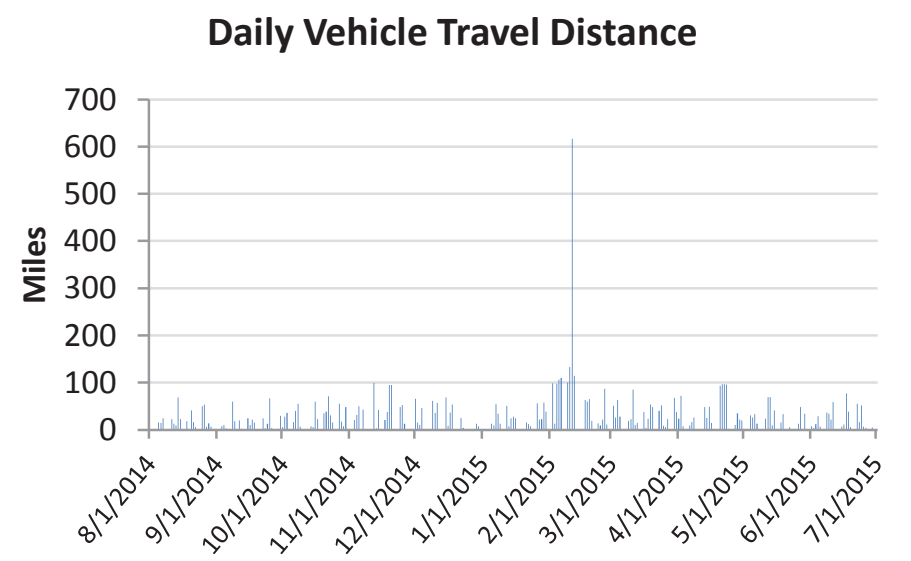

198

6.0

36.2

1187

7168.7

16758

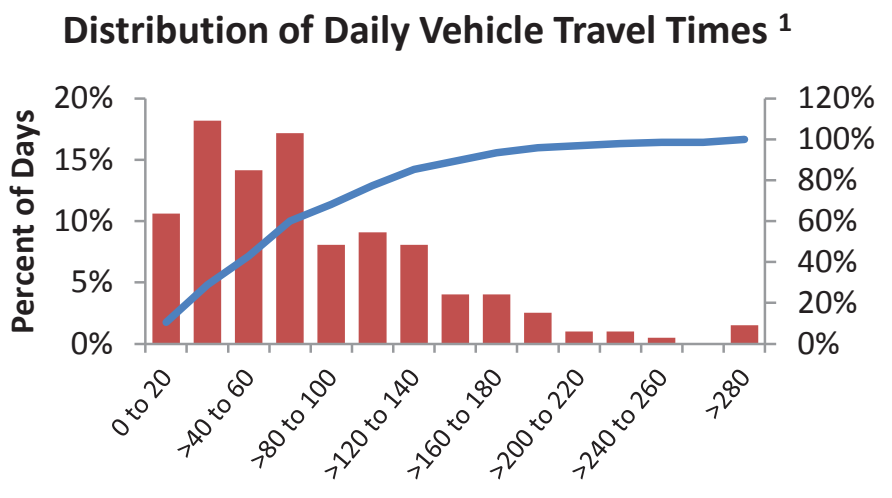

Daily Vehicle Trip Times (min)

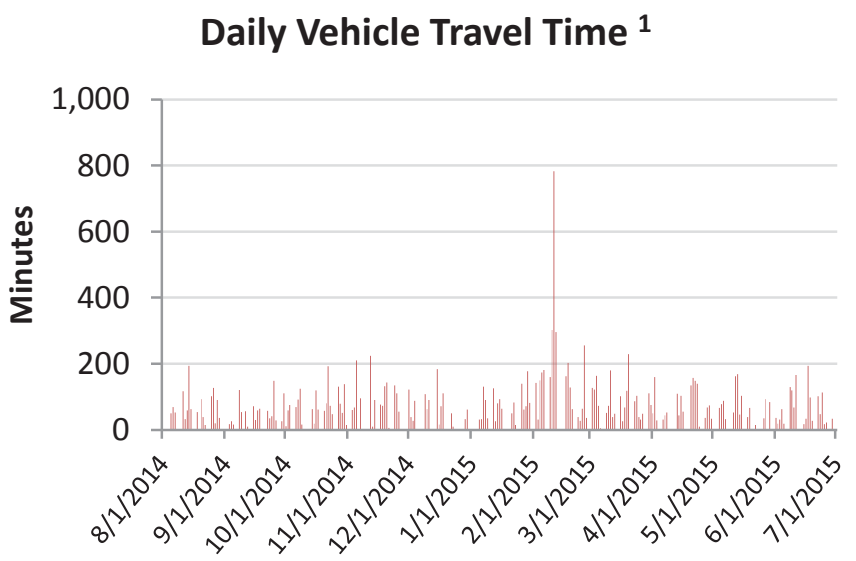

Note 1: Travel time includes all time when vehicle is in the "key on" state, even when the vehicle is not moving

$\begin{array}{ccc}\text { EV Range } & \begin{array}{c}\text { Percentage of } \\ \text { days vehicle } \\ \text { exceeds EV range }\end{array} & \begin{array}{c}\text { Number of days } \\ \text { vehicle exceeds } \\ \text { EV range }\end{array} \\ 63 & 17 \% & 34 \\ 67 & 15 \% & 30 \\ 63 & 13 \% & 25\end{array}$


Vehicle:

Report Period:

Model Year:

Vehicle Make:

Vehicle Model:

Body Type:
5L-G106501H

8/1/2014 00:00:00 - 7/1/2015 00:00:00

2009

Pontiac

G6

sedan
Total Number of Days with Driving

Average Number of Trips

Average Trip Distance

Total Number of Trips

Total Distance (miles)

Total Trip Duration (minutes)
107

2.9

12.5

308

1339.5

3087

\section{Distribution of Daily Vehicle Travel Distance}

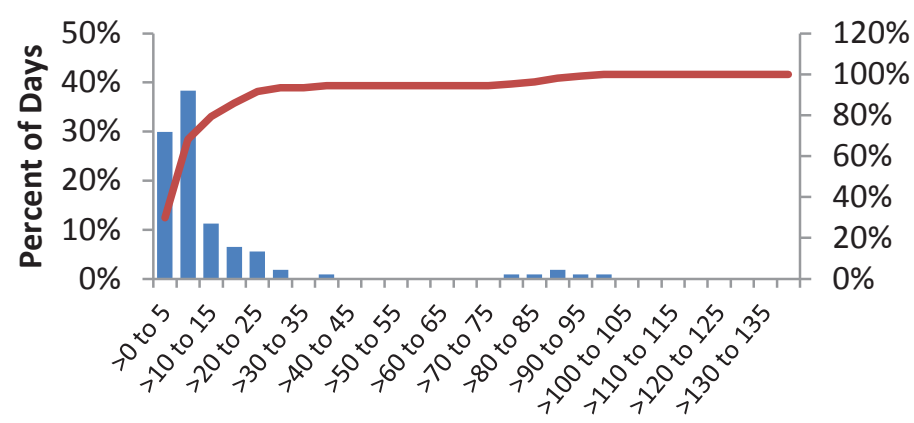

Daily Vehicle Miles Traveled

\section{Daily Vehicle Travel Distance}

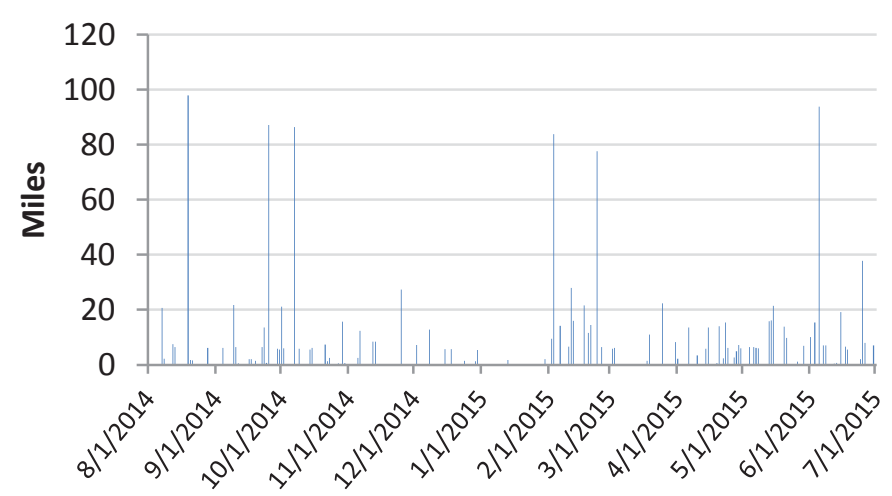

Distribution of Daily Vehicle Travel Times ${ }^{1}$

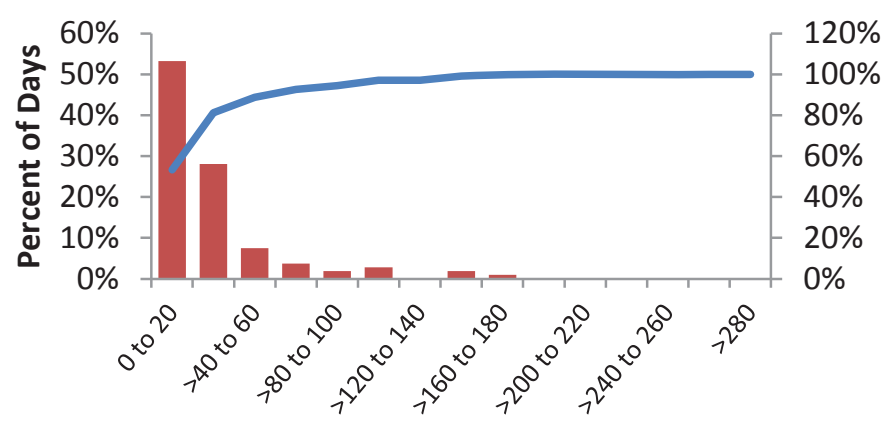

Daily Vehicle Trip Times (min)

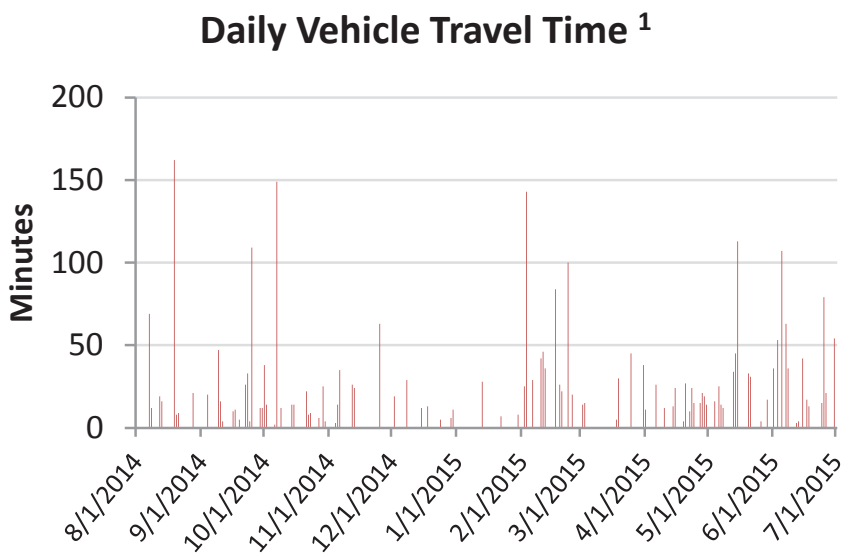

Note 1: Travel time includes all time when vehicle is in the "key on" state, even when the vehicle is not moving

$\begin{array}{cc}\text { Percentage of } & \text { Number of days } \\ \text { days vehicle } & \text { vehicle exceeds }\end{array}$

exceeds EV range EV range

EV Range

59

63

$6 \%$

$6 \%$

$6 \%$
6

6

6 
Vehicle:

Report Period:

Model Year:

Vehicle Make:

Vehicle Model:

Body Type:
5L-G106208H

8/1/2014 00:00:00 - 7/1/2015 00:00:00

2009

Pontiac

G6

sedan
Total Number of Days with Driving Average Number of Trips

Average Trip Distance

Total Number of Trips

Total Distance (miles)

Total Trip Duration (minutes)
90

4.7

67.5

420

6072.2

10690

\section{Distribution of Daily Vehicle Travel Distance}

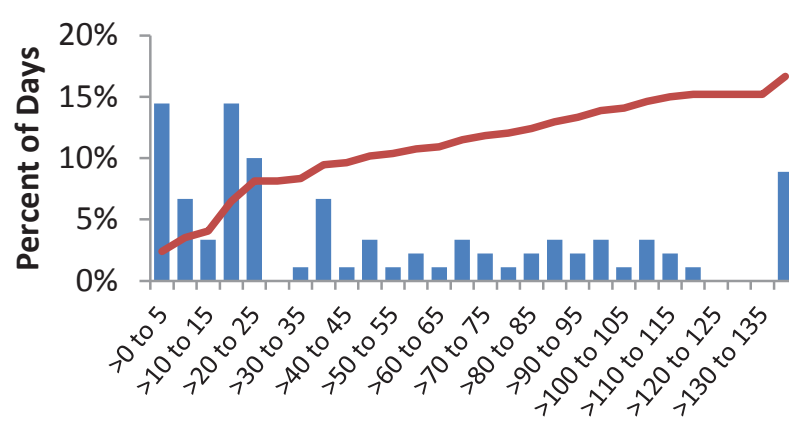

Daily Vehicle Miles Traveled

\section{Daily Vehicle Travel Distance}

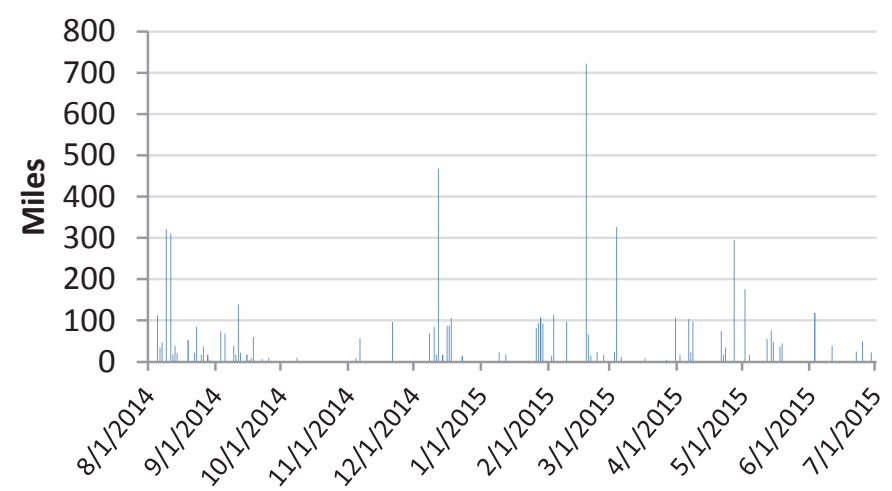

Distribution of Daily Vehicle Travel Times ${ }^{1}$

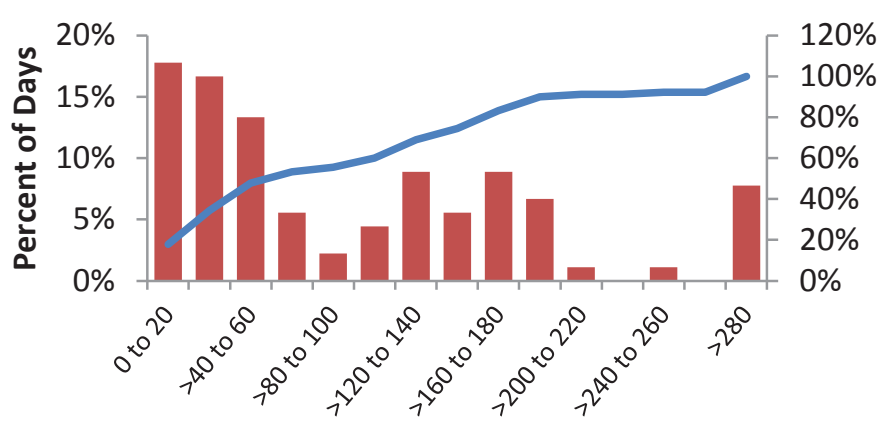

Daily Vehicle Trip Times (min)

\section{Daily Vehicle Travel Time ${ }^{1}$}

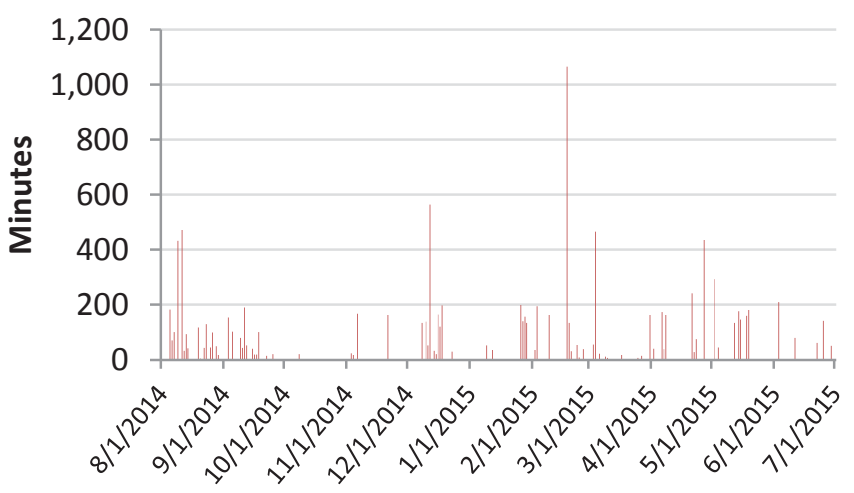

Note 1: Travel time includes all time when vehicle is in the "key on" state, even when the vehicle is not moving

$\begin{array}{cc}\text { Percentage of } & \text { Number of days } \\ \text { days vehicle } & \text { vehicle exceeds }\end{array}$

EV Range

59

63

67 exceeds EV range

$36 \%$

$34 \%$

$33 \%$
EV range

32

31

30 
Vehicle:

Report Period:

Model Year:

Vehicle Make:

Vehicle Model:

Body Type:
5L-G106206H

8/1/2014 00:00:00 - 7/1/2015 00:00:00

2009

Pontiac

G6

sedan
Total Number of Days with Driving Average Number of Trips

Average Trip Distance

Total Number of Trips

Total Distance (miles)

Total Trip Duration (minutes)
170

5.3

58.1

898

9885.2

18270

\section{Distribution of Daily Vehicle Travel Distance}

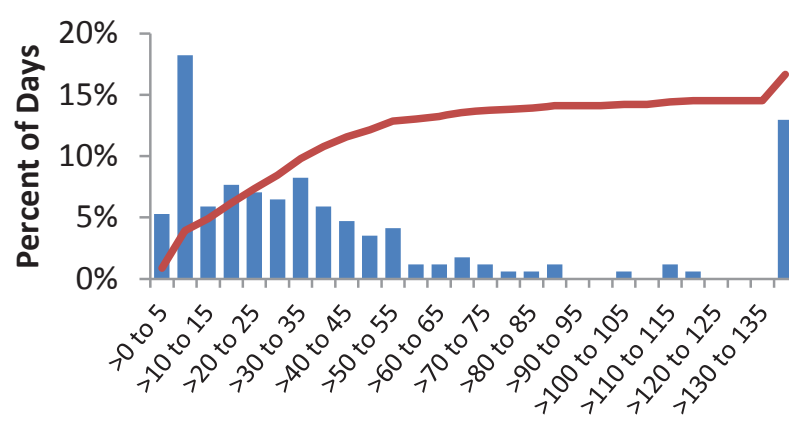

Daily Vehicle Miles Traveled

\section{Daily Vehicle Travel Distance}

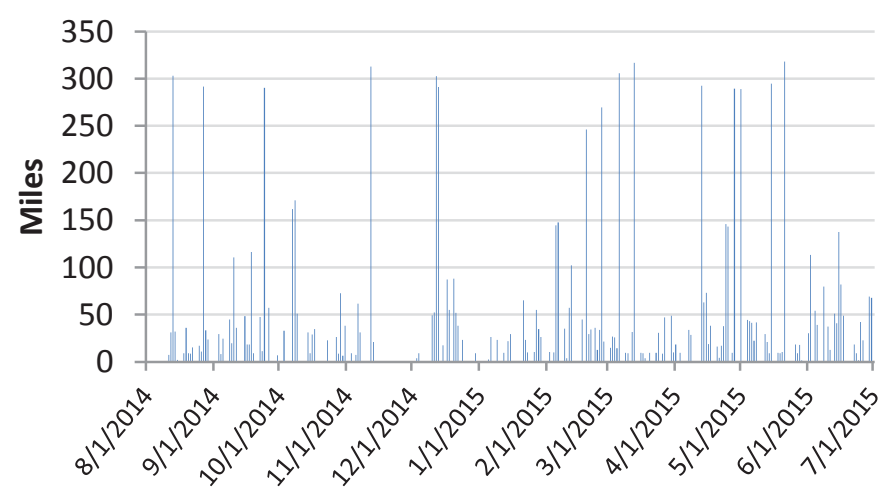

Distribution of Daily Vehicle Travel Times ${ }^{1}$

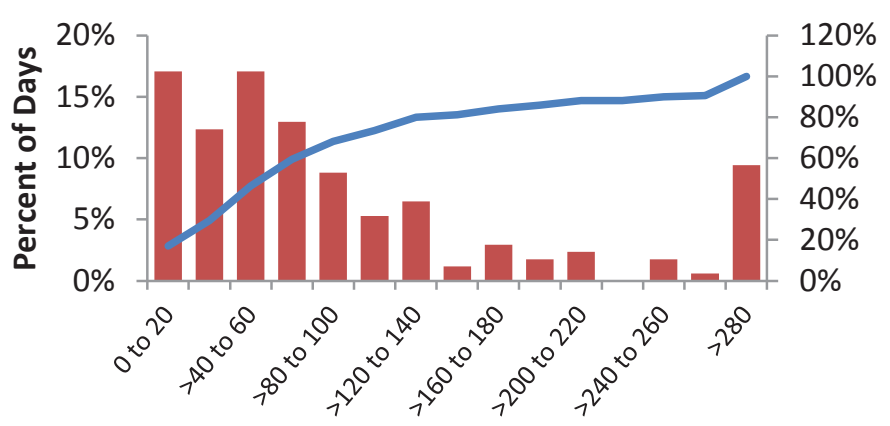

Daily Vehicle Trip Times (min)

\section{Daily Vehicle Travel Time ${ }^{1}$}

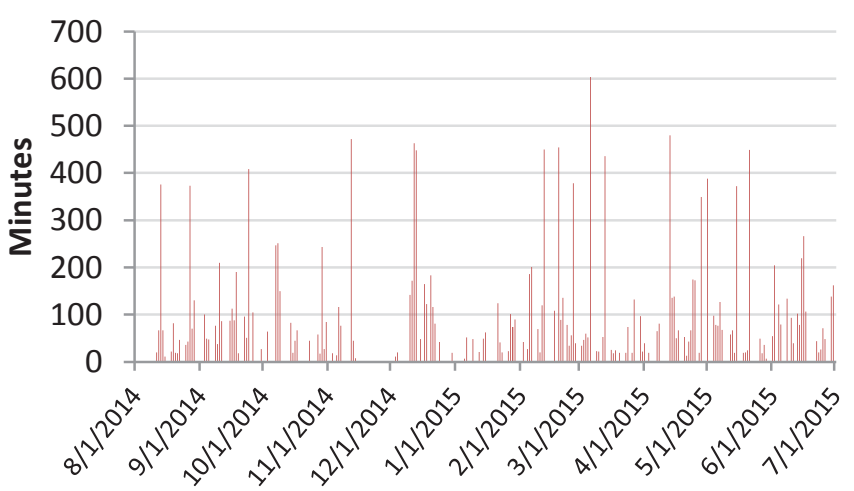

Note 1: Travel time includes all time when vehicle is in the "key on" state, even when the vehicle is not moving

$\begin{array}{cc}\text { Percentage of } & \text { Number of days } \\ \text { days vehicle } & \text { vehicle exceeds }\end{array}$

EV Range

59

63

67 exceeds EV range

$22 \%$

$21 \%$

$20 \%$
EV range

37

36

34 
Vehicle:

Report Period:

Model Year:

Vehicle Make:

Vehicle Model:

Body Type:

Total Number of Days with Driving

Average Number of Trips

Average Trip Distance

Total Number of Trips

Total Distance (miles)

Total Trip Duration (minutes)
$5 L-294277$

8/1/2014 00:00:00 - 7/1/2015 00:00:00

2009

Chevy

Malibu

sedan

\section{Distribution of Daily Vehicle Travel Distance}

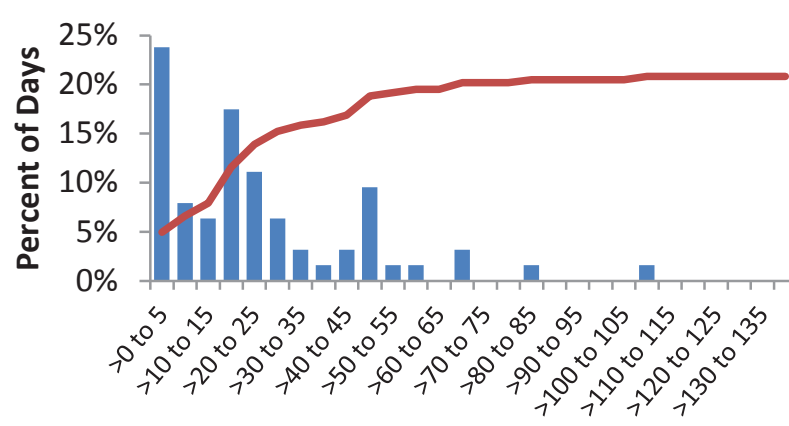

Daily Vehicle Miles Traveled

\section{Daily Vehicle Travel Distance}

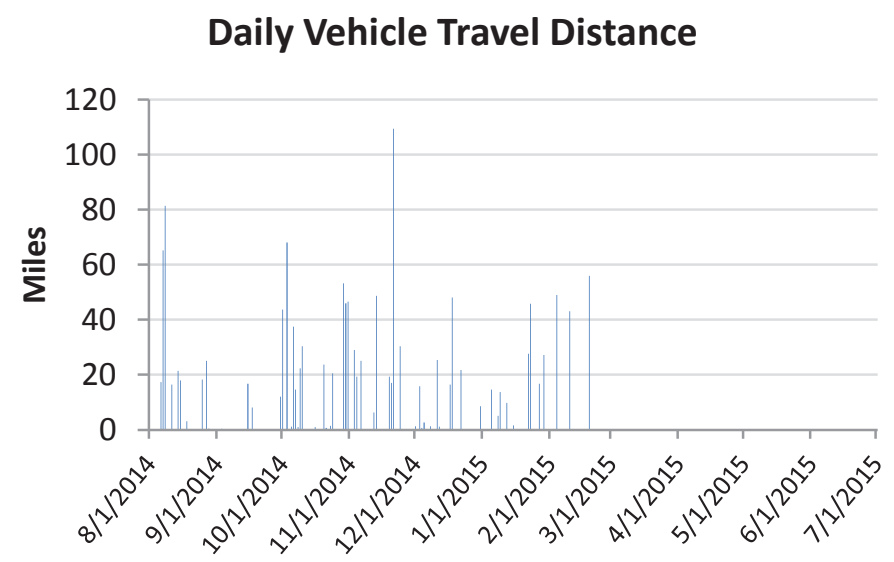

$120 \%$

$100 \%$

$80 \%$

$60 \%$

$40 \%$

$20 \%$

$0 \%$
63

4.8

23.4

300

1472.7

4212

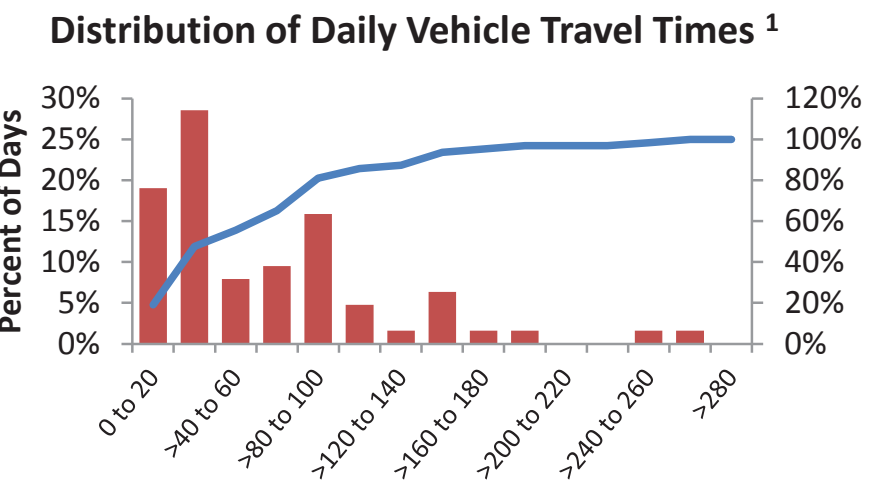

Daily Vehicle Trip Times (min)

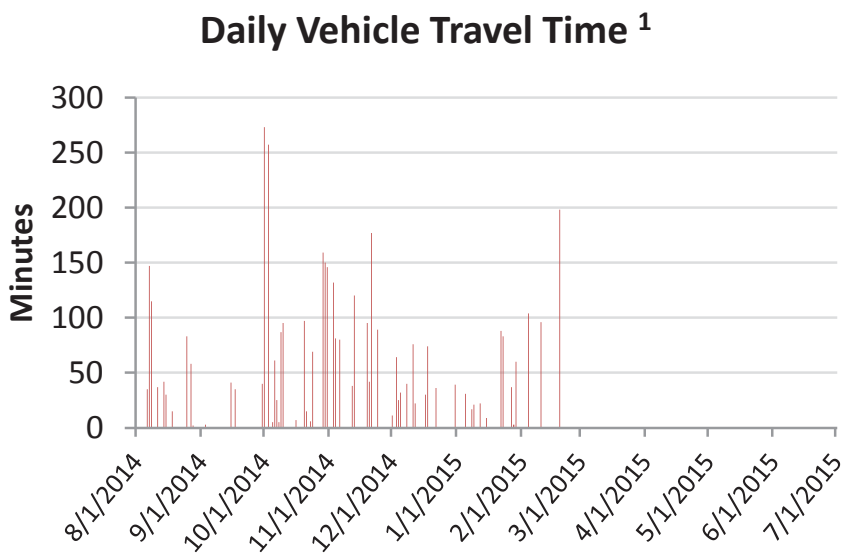

Note 1: Travel time includes all time when vehicle is in the "key on" state, even when the vehicle is not moving

$\begin{array}{cc}\text { Percentage of } & \text { Number of days } \\ \text { days vehicle } & \text { vehicle exceeds }\end{array}$

EV Range

59

63

67 exceeds EV range

$6 \%$

$6 \%$

$5 \%$
EV range

4

4

3 
Vehicle:

Report Period:

Model Year:

Vehicle Make:

Vehicle Model:

Body Type:

Total Number of Days with Driving

Average Number of Trips

Average Trip Distance

Total Number of Trips

Total Distance (miles)

Total Trip Duration (minutes)
5L-294274

8/1/2014 00:00:00 - 7/1/2015 00:00:00

2009

Chevy

Malibu

sedan

\section{Distribution of Daily Vehicle Travel Distance}

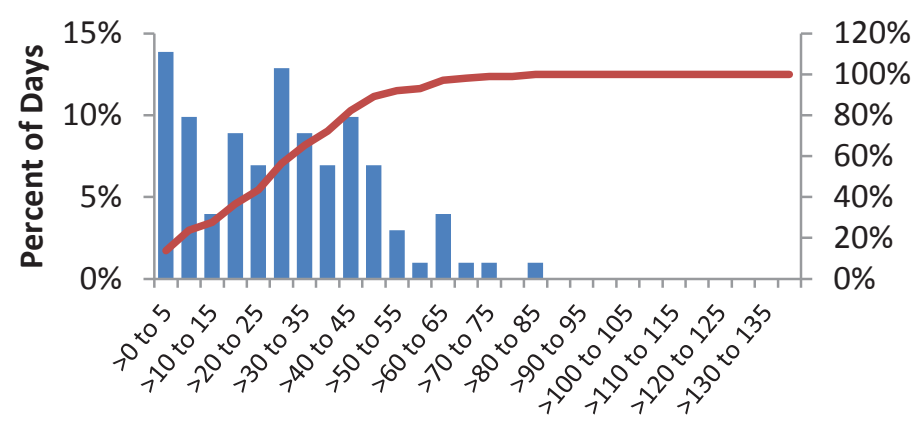

Daily Vehicle Miles Traveled

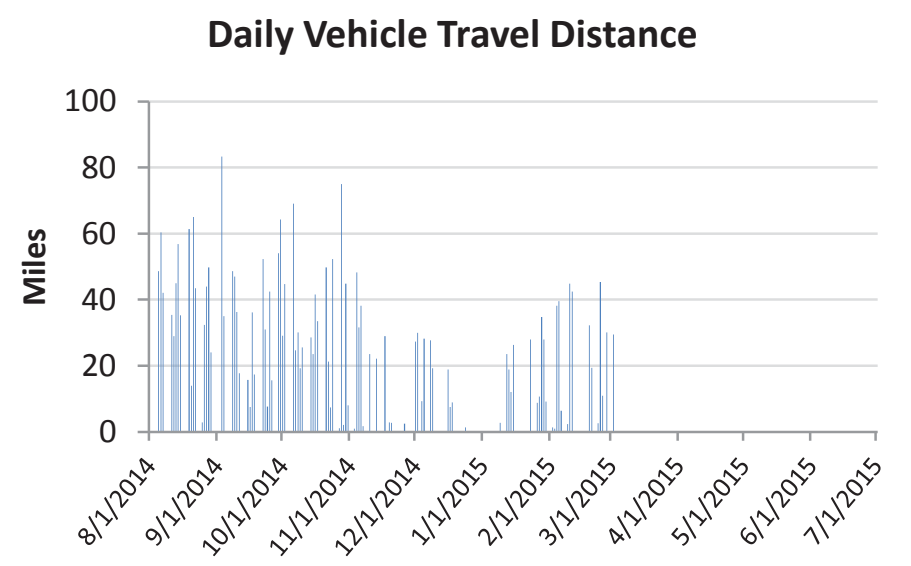

101

8.0

28.3

804

2862.4

6167
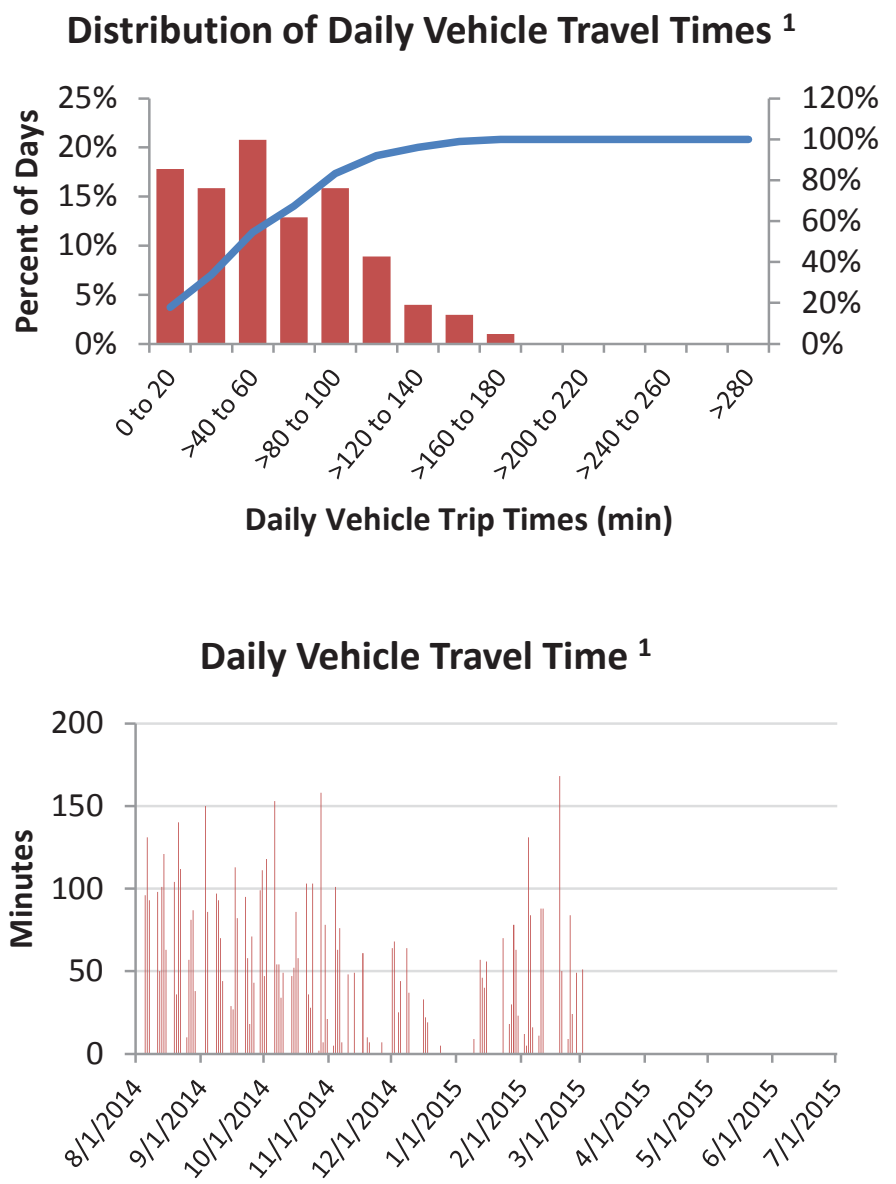

Note 1: Travel time includes all time when vehicle is in the "key on" state, even when the vehicle is not moving

$\begin{array}{ccc}\text { EV Range } & \begin{array}{c}\text { Percentage of } \\ \text { days vehicle } \\ \text { exceeds EV range }\end{array} & \begin{array}{c}\text { Number of days } \\ \text { vehicle exceeds } \\ \text { EV range }\end{array} \\ 59 & 7 \% & 7 \\ 63 & 5 \% & 5 \\ 67 & 3 \% & 3\end{array}$


Vehicle:

Report Period:

Model Year:

Vehicle Make:

Vehicle Model:

Body Type:

Total Number of Days with Driving

Average Number of Trips

Average Trip Distance

Total Number of Trips

Total Distance (miles)

Total Trip Duration (minutes)
$5 L-294267$

8/1/2014 00:00:00 - 7/1/2015 00:00:00

2009

Chevy

Malibu

sedan

\section{Distribution of Daily Vehicle Travel Distance}

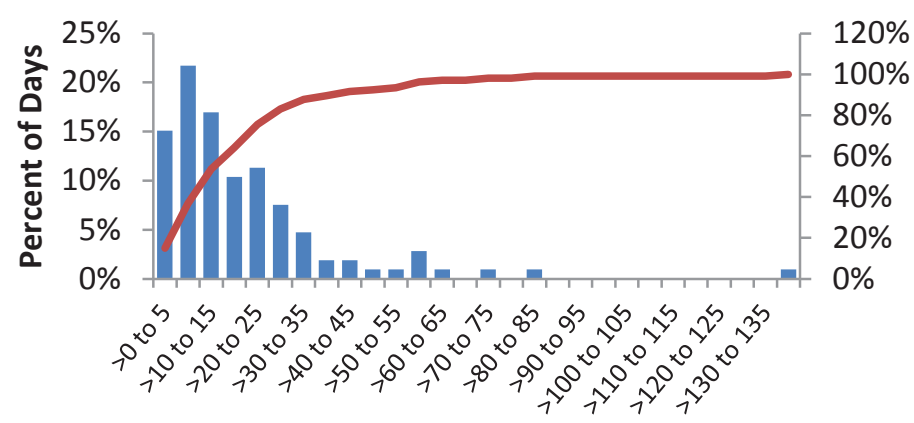

Daily Vehicle Miles Traveled

\section{Daily Vehicle Travel Distance}

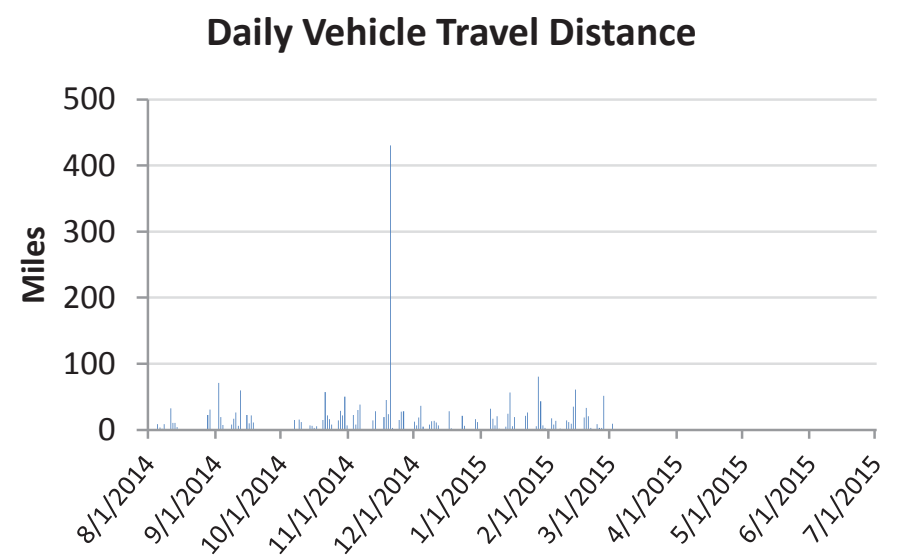

106

8.8

22.3

932

2360.0

6742

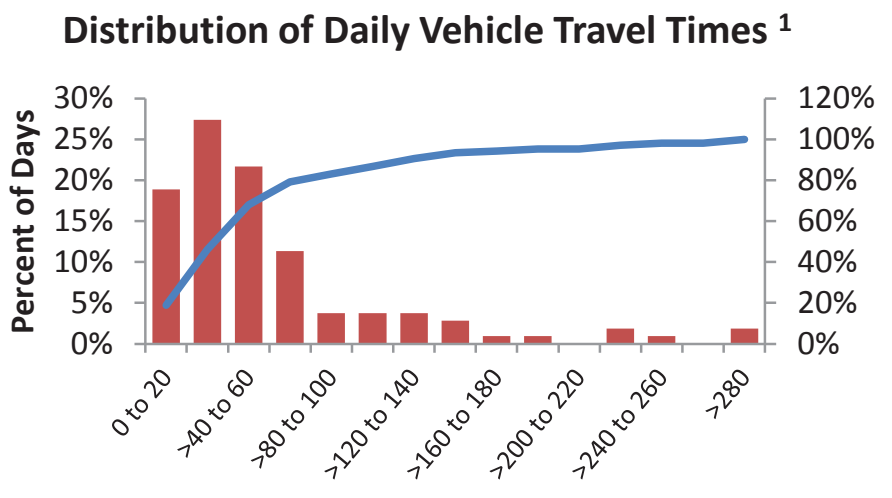

Daily Vehicle Trip Times (min)

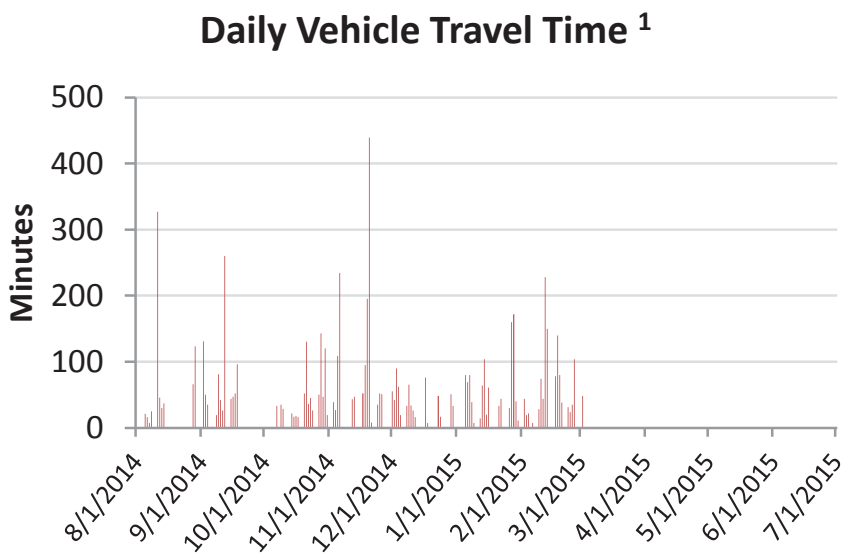

Note 1: Travel time includes all time when vehicle is in the "key on" state, even when the vehicle is not moving

$\begin{array}{cc}\text { Percentage of } & \text { Number of days } \\ \text { days vehicle } & \text { vehicle exceeds }\end{array}$

EV Range

59

63

67 exceeds EV range

$5 \%$

$3 \%$

$3 \%$
EV range

\section{5}

3

3 
Vehicle:

Report Period:

Model Year:

Vehicle Make:

Vehicle Model:

Body Type:

Total Number of Days with Driving

Average Number of Trips

Average Trip Distance

Total Number of Trips

Total Distance (miles)

Total Trip Duration (minutes)
5L-294266

8/1/2014 00:00:00 - 7/1/2015 00:00:00

2009

Chevy

Malibu

sedan
113

9.9

32.3

1120

3647.3

9575

\section{Distribution of Daily Vehicle Travel Distance}

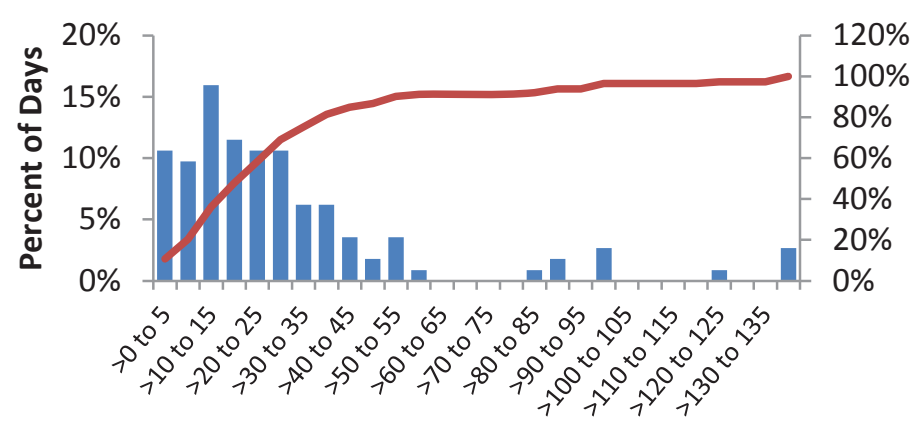

Daily Vehicle Miles Traveled

\section{Daily Vehicle Travel Distance}

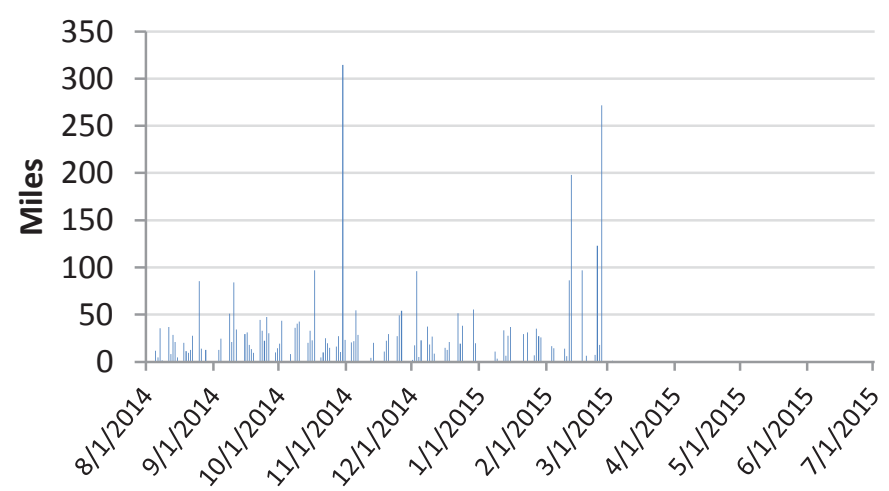

Distribution of Daily Vehicle Travel Times ${ }^{1}$

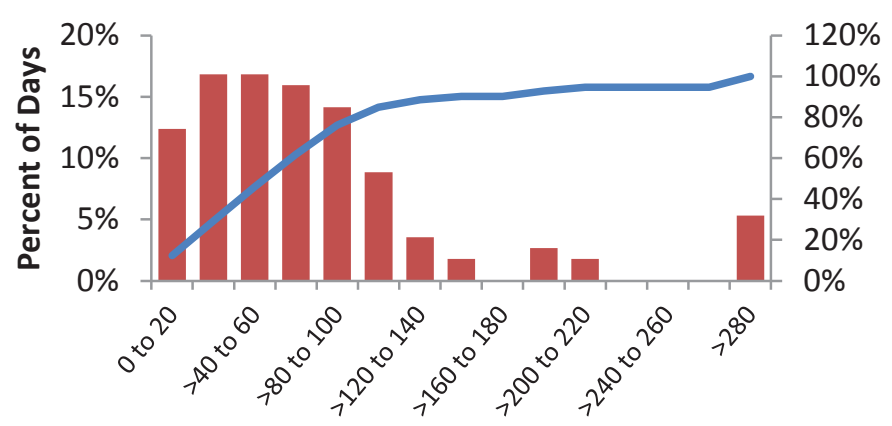

Daily Vehicle Trip Times (min)

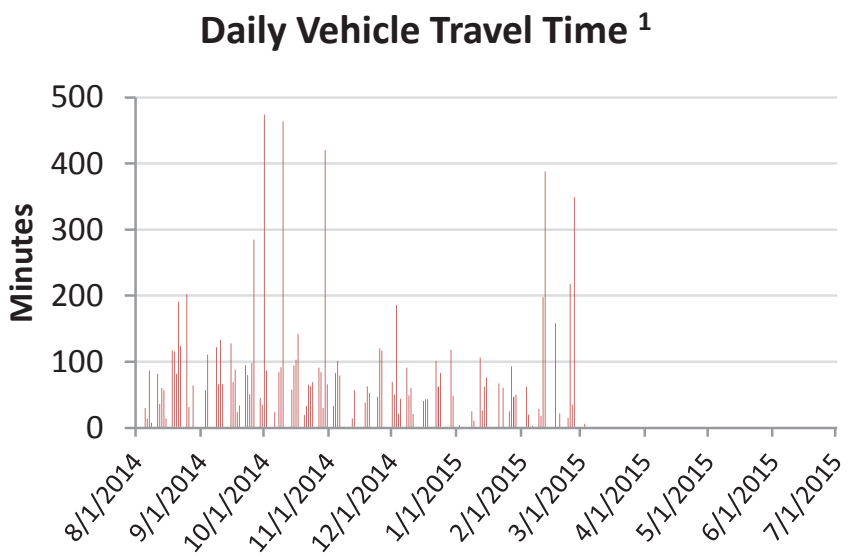

Note 1: Travel time includes all time when vehicle is in the "key on" state, even when the vehicle is not moving

$\begin{array}{cc}\text { Percentage of } & \text { Number of days } \\ \text { days vehicle } & \text { vehicle exceeds }\end{array}$

EV Range

59

63

67 exceeds EV range

$9 \%$

$9 \%$

$9 \%$
EV range

10

10

10 
Vehicle:

Report Period:

Model Year:

Vehicle Make:

Vehicle Model:

Body Type:

Total Number of Days with Driving

Average Number of Trips

Average Trip Distance

Total Number of Trips

Total Distance (miles)

Total Trip Duration (minutes)
5B-G414199H

8/1/2014 00:00:00 - 7/1/2015 00:00:00

2009

Dodge

Dakota

small pickup

\section{Distribution of Daily Vehicle Travel Distance}

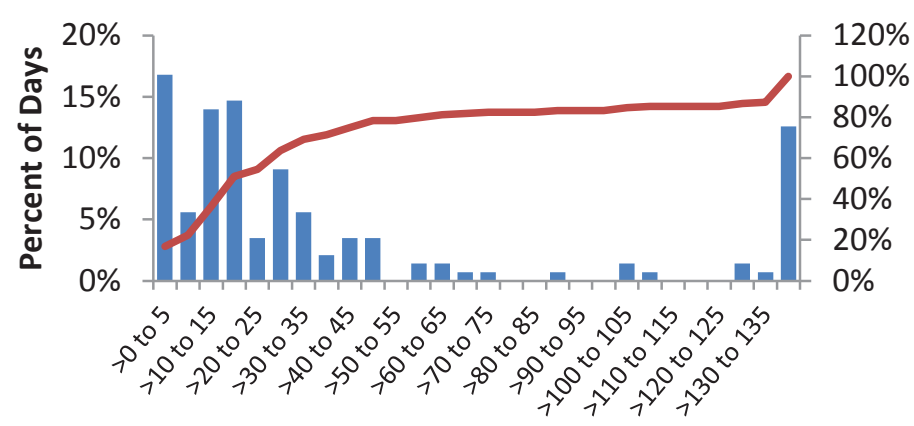

Daily Vehicle Miles Traveled

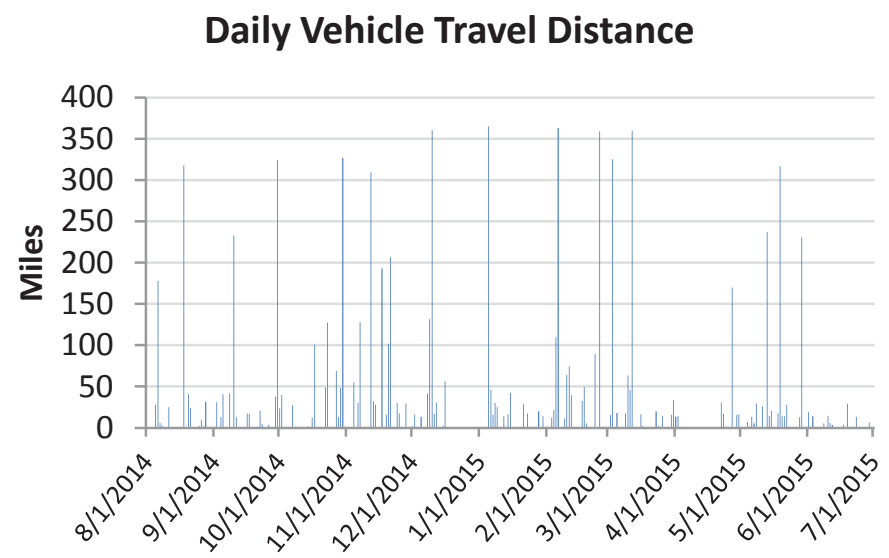

143

5.2

58.4

744

8356.4

16233

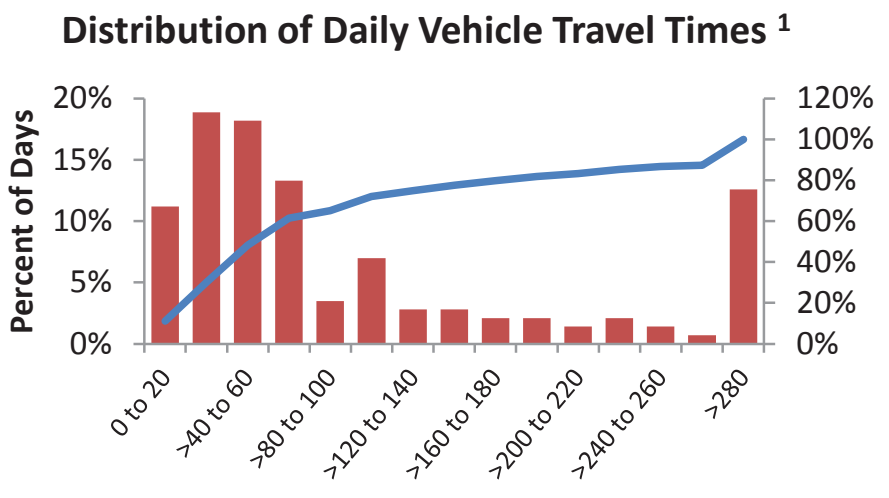

Daily Vehicle Trip Times (min)

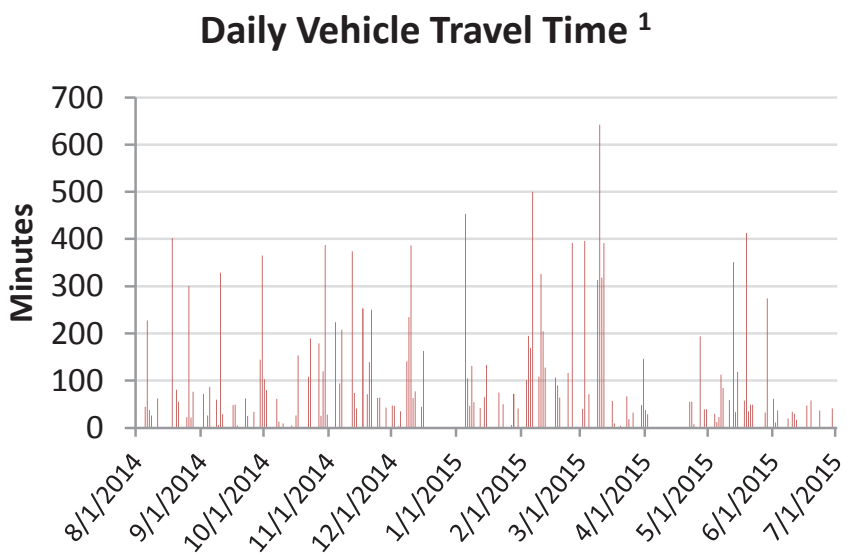

Note 1: Travel time includes all time when vehicle is in the "key on" state, even when the vehicle is not moving

$\begin{array}{ccc}\text { EV Range } & \begin{array}{c}\text { Percentage of } \\ \text { days vehicle } \\ \text { exceeds EV range }\end{array} & \begin{array}{c}\text { Number of days } \\ \text { vehicle exceeds } \\ \text { EV range }\end{array} \\ 63 & 20 \% & 29 \\ 67 & 20 \% & 29 \\ 63 & 19 \% & 27\end{array}$


Vehicle:

Report Period:

Model Year:

Vehicle Make:

Vehicle Model:

Body Type:
5B-G413964H

8/1/2014 00:00:00 - 7/1/2015 00:00:00

2009

Dodge

Caravan

van
Total Number of Days with Driving

1

0.0

0.0

Average Trip Distance

Total Number of Trips

Total Distance (miles)

Total Trip Duration (minutes)
Distribution of Daily Vehicle Travel Times ${ }^{1}$

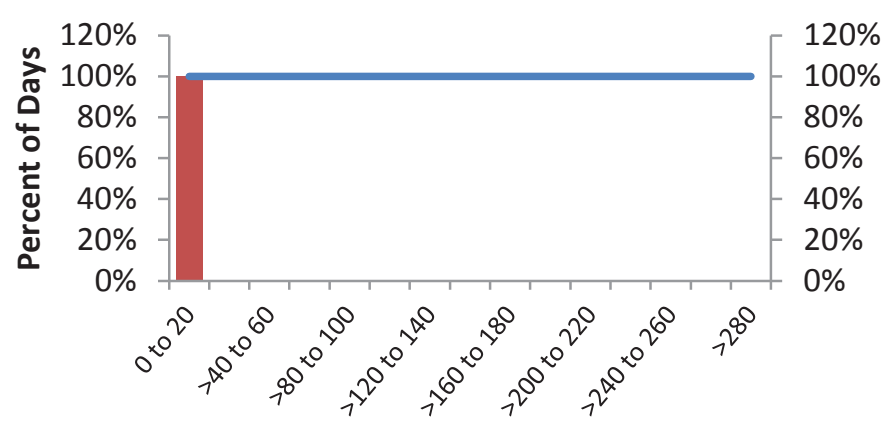

Daily Vehicle Trip Times (min)

\section{Daily Vehicle Travel Time ${ }^{1}$}

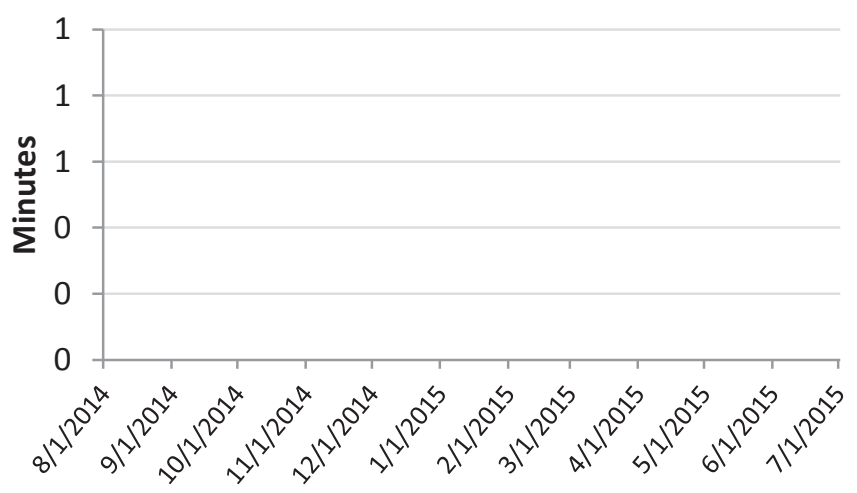

Note 1: Travel time includes all time when vehicle is in the "key on" state, even when the vehicle is not moving

Percentage of Number of days
days vehicle

EV Range

59

63

67 exceeds EV range

$0 \%$

$0 \%$

$0 \%$
EV range

0

0

0 
Vehicle:

Report Period:

Model Year:

Vehicle Make:

Vehicle Model:

Body Type:

Total Number of Days with Driving

Average Number of Trips

Average Trip Distance

Total Number of Trips

Total Distance (miles)

Total Trip Duration (minutes)
5B-G410523G

8/1/2014 00:00:00 - 7/1/2015 00:00:00

2008

Chevrolet

Colorado

small pickup

\section{Distribution of Daily Vehicle Travel Distance}

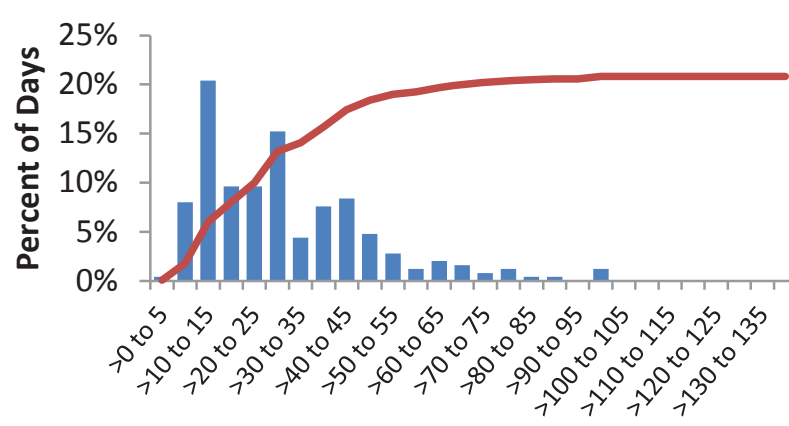

Daily Vehicle Miles Traveled

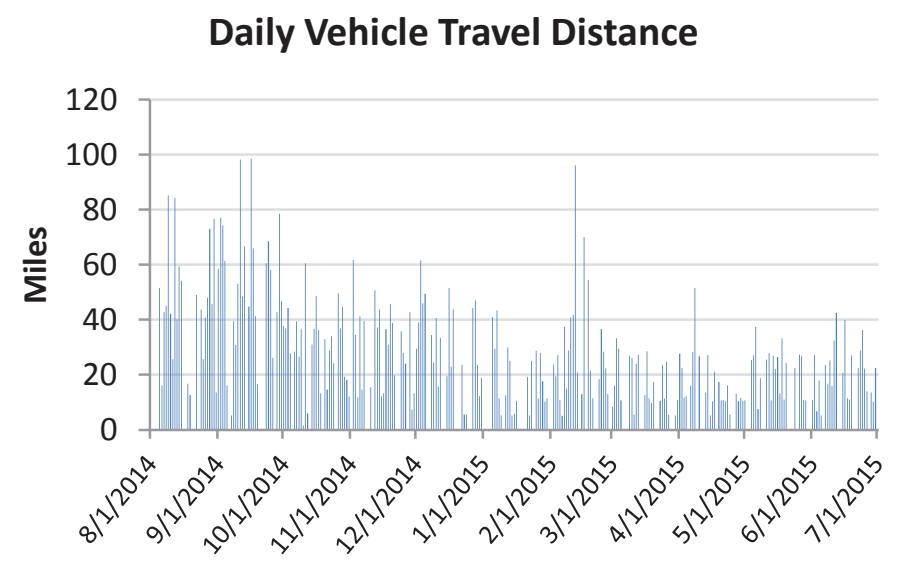

250

7.7

29.0

1923

7239.3

33433
$120 \%$

$100 \%$

$80 \%$

$60 \%$

$40 \%$

$20 \%$

$0 \%$

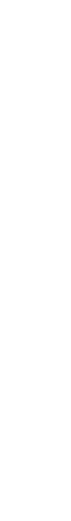

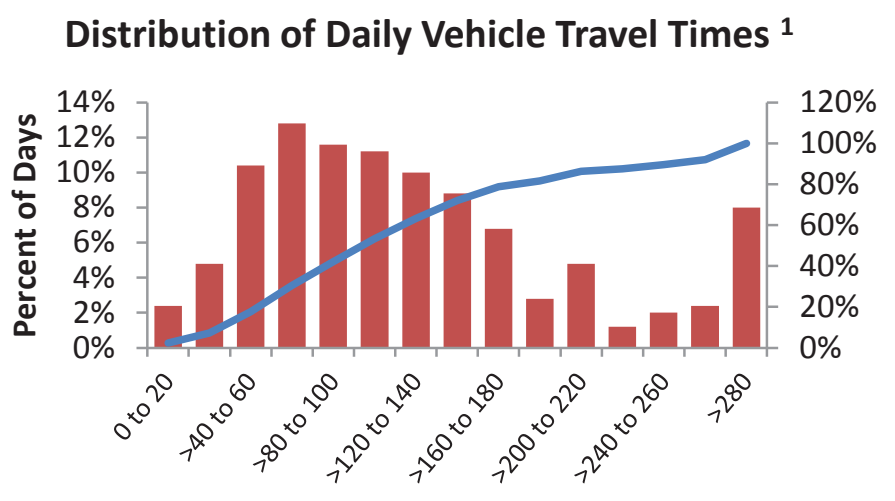

Daily Vehicle Trip Times (min)

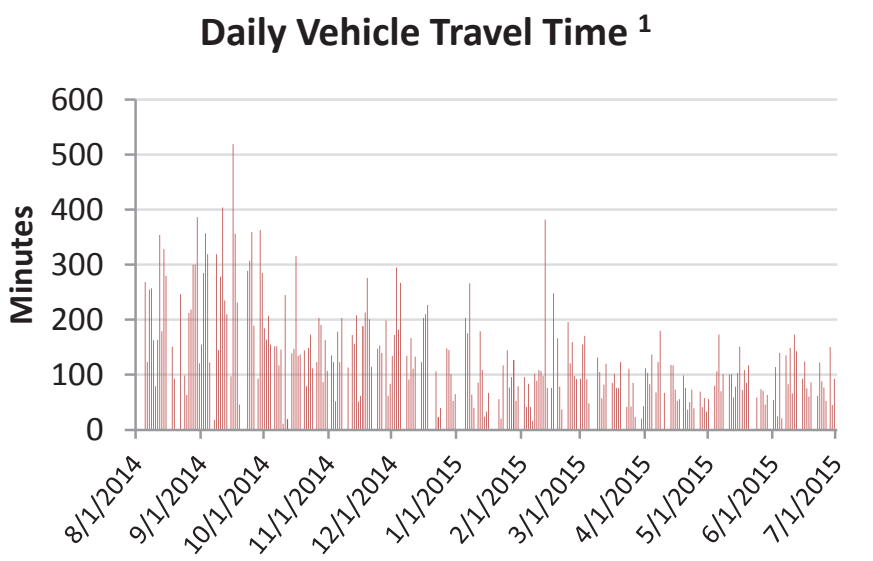

Note 1: Travel time includes all time when vehicle is in the "key on" state, even when the vehicle is not moving

$\begin{array}{ccc}\text { EV Range } & \begin{array}{c}\text { Percentage of } \\ \text { days vehicle } \\ \text { exceeds EV range }\end{array} & \begin{array}{c}\text { Number of days } \\ \text { vehicle exceeds } \\ \text { EV range }\end{array} \\ 59 & 8 \% & 20 \\ 63 & 6 \% & 14 \\ 67 & 5 \% & 12\end{array}$


Vehicle:

Report Period:

Model Year:

Vehicle Make:

Vehicle Model:

Body Type:
5B-G410512G

8/1/2014 00:00:00 - 7/1/2015 00:00:00

2008

Chevrolet

Uplander LS

van

Total Number of Days with Driving

Average Number of Trips

Average Trip Distance

Total Number of Trips

1619

Total Distance (miles)

5782.9

Total Trip Duration (minutes)

\section{Distribution of Daily Vehicle Travel Distance}

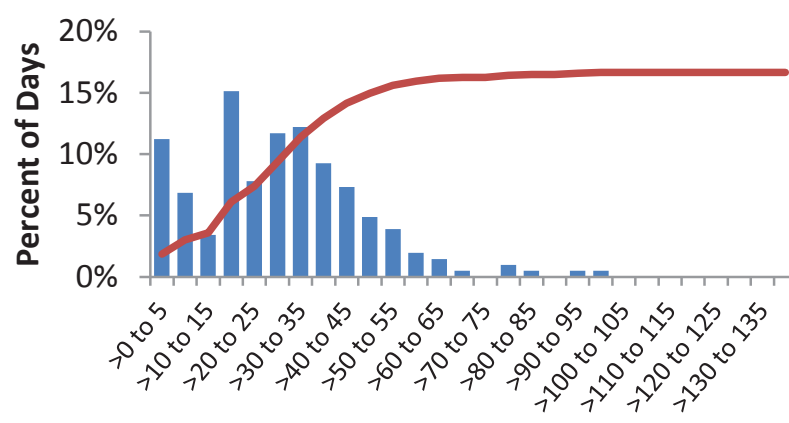

Daily Vehicle Miles Traveled

\section{Daily Vehicle Travel Distance}

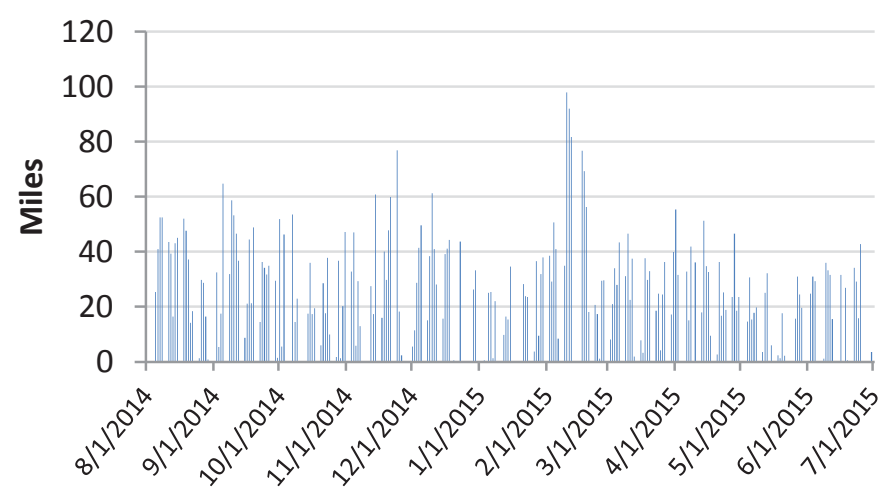

Distribution of Daily Vehicle Travel Times ${ }^{1}$

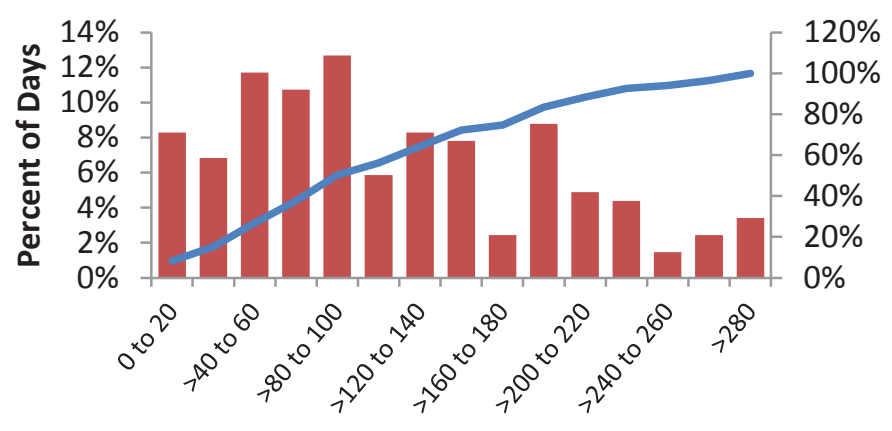

Daily Vehicle Trip Times (min)

\section{Daily Vehicle Travel Time ${ }^{1}$}

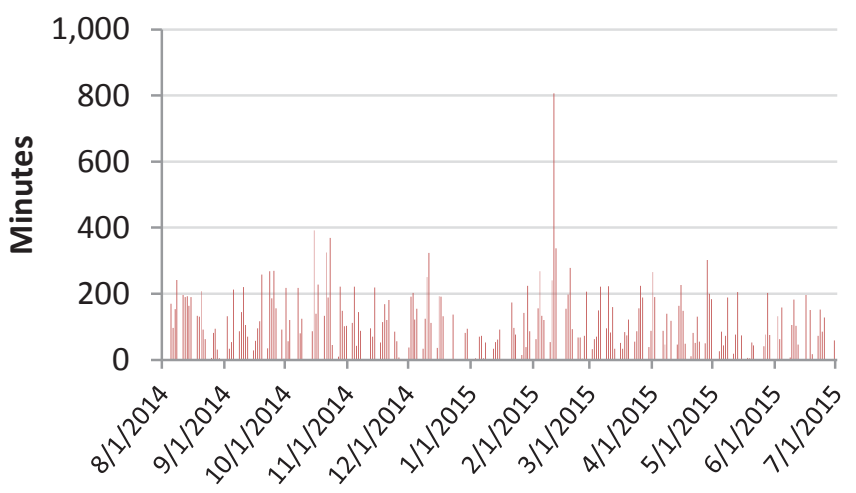

Note 1: Travel time includes all time when vehicle is in the "key on" state, even when the vehicle is not moving

$\begin{array}{cc}\text { Percentage of } & \text { Number of days } \\ \text { days vehicle } & \text { vehicle exceeds }\end{array}$

EV Range

59

63

67 exceeds EV range

$5 \%$

$3 \%$

$3 \%$
EV range

10

7

6 